The copyright of this thesis vests in the author. No quotation from it or information derived from it is to be published without full acknowledgement of the source. The thesis is to be used for private study or noncommercial research purposes only.

Published by the University of Cape Town (UCT) in terms of the non-exclusive license granted to UCT by the author. 


\title{
Flotation in a Novel Oscillatory Baffled Column
}

\author{
by

\section{Christopher James Anderson} \\ B.Sc. Chemical Engineering (University of Cape Town)
}

A Thesis Presented for the Degree of DOCTOR OF PHILOSOPHY

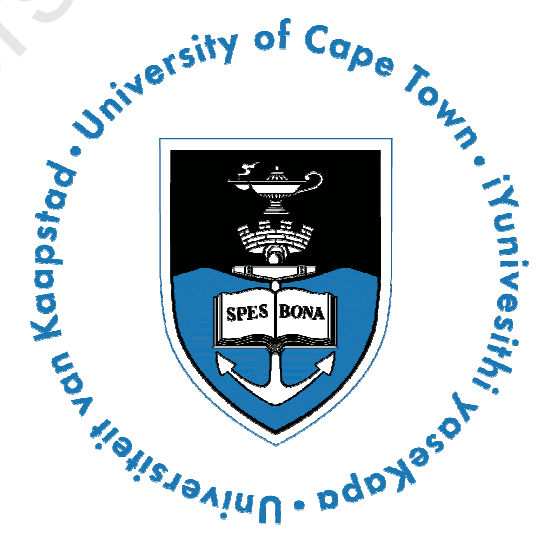

Department of Chemical Engineering University of Cape Town

April 2008 


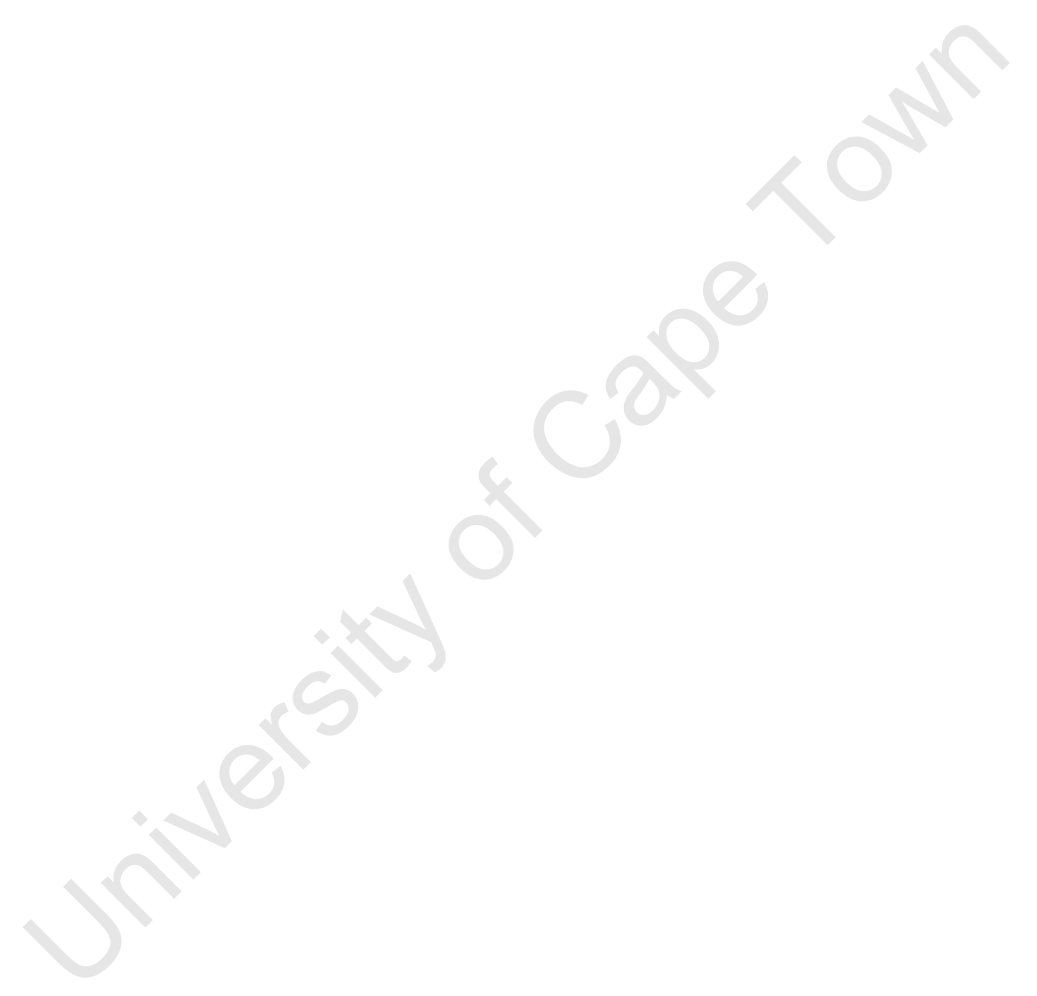


I hereby certify that the work embodied in this thesis is the result of original research and has not been submitted for another degree at any other university or institution.

Christopher Anderson

April 2008 


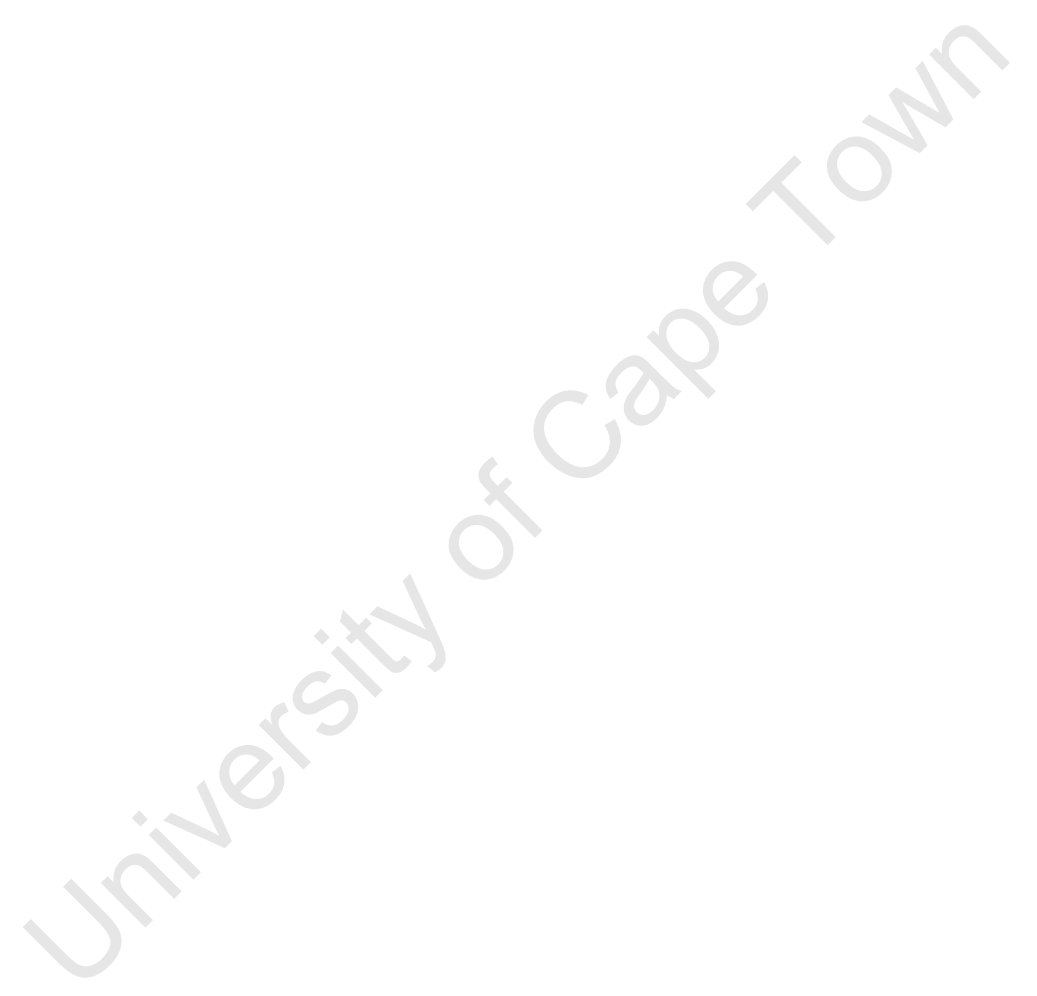




\section{Acknowledgments}

I would like to express my sincere gratitude to my supervisor, David Deglon, and my co-supervisor, Martin Harris, for their invaluable guidance, enthusiasm and good humour throughout my $\mathrm{PhD}$ candidature.

The help of the following people and organisations is also gratefully acknowledged:

- The AMIRA P9N project, the Department of Labour National Research Foundation and the Department of Chemical Engineering at UCT for their combined assistance in financing this project.

- Bill Randall, Peter Dobias and Kenneth Maseko for their assistance in the construction of the experimental apparatus used in this study.

- The sponsors of the AMIRA P9N project for providing the opportunity to test the $\mathrm{OBC}$ in an industrial environment.

- Kudzai Changunda and Siyabonga Xozwa for their tireless hard work during the experimental phases of this project.

- My fiancée, Jennifer, for her tremendous support and encouragement throughout this study. 


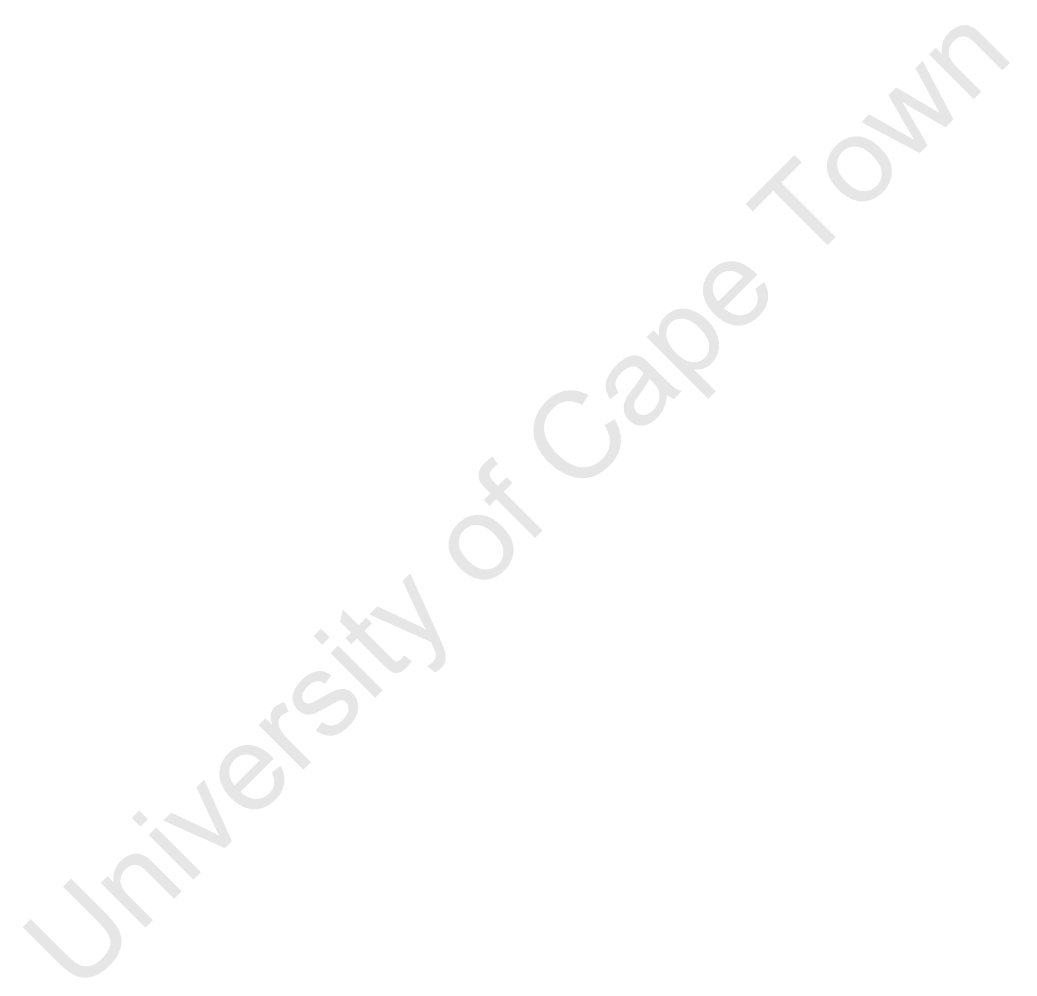




\section{Publications}

\section{Journal Publications}

Anderson, C. J., Deglon, D. A. \& Harris, M. C., A Novel Device for Fine Particle Flotation: the Oscillatory Baffled Column. Minerals Engineering (Submitted for Publication).

\section{Conference Papers (Refereed)}

Anderson, C. J., Deglon, D. A. \& Harris, M. C., The effect of energy on quartz flotation in a novel oscillatory baffled column. Proceedings of the XXIIIth International Minerals Processing Congress, Istanbul. (2006). 


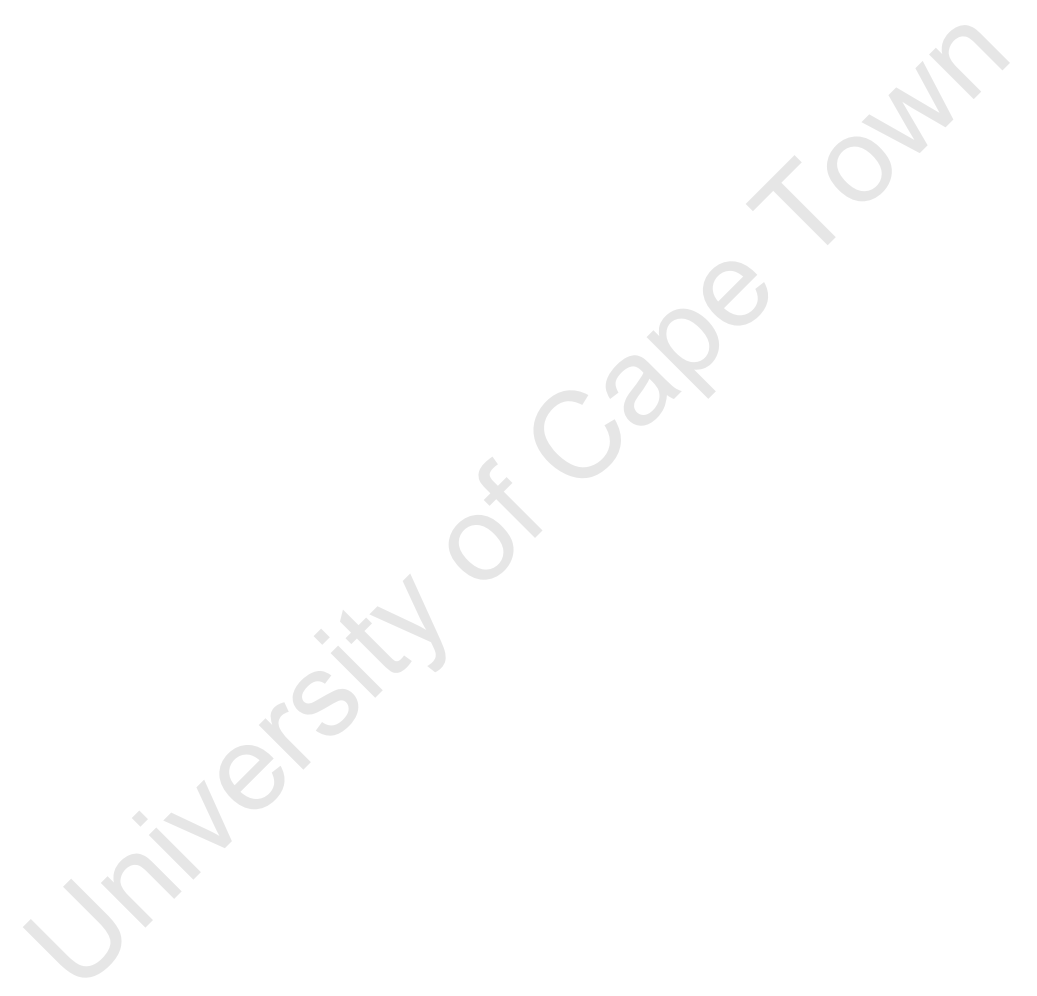




\section{Synopsis}

This thesis presents an evaluation of an Oscillatory Baffled Column as a novel flotation device and as a research tool for investigating the role which hydrodynamics play in promoting particle-bubble contacting. The cell differs from a conventional cell design in that bubble generation is decoupled from the power input. This allows the hydrodynamic environment to be optimised for effective particlebubble contacting. The design also incorporates a novel mechanism of agitation which provides a more even distribution of shear rate than would be obtained in a stirred system and allows considerably more variation of the power input to the cell. Based on these properties it is hypothesised that the $\mathrm{OBC}$ would provide an effective hydrodynamic environment for fine particle flotation, particularly in highly viscous, non-Newtonian slurries. It is also hypothesised that the development of such a cell technology will facilitate an improved understanding of the role which hydrodynamics play in promoting particle-bubble contacting.

To investigate these hypotheses, the $\mathrm{OBC}$ was first characterised in terms of key flotation parameters, such as bubble size, gas hold-up and mixing. The cell was then flotation tested on quartz, in order to benchmark the study against previous studies on hydrodynamics and particle-bubble contacting in stirred systems. Finally, the OBC was tested in application to a highly viscous, non-Newtonian nickel ore slimes slurry which is difficult to treat using conventional cell technologies. This study gave context to the potential future role of $\mathrm{OBC}$ technology in the minerals processing industry. 
The results of this thesis clearly show that the $\mathrm{OBC}$ is a promising novel technology for the treatment of fine mineral slurries. The cell has been demonstrated to significantly improve flotation kinetics by a factor of between 1.4 and 1.6 relative to a standard column. Optimal flotation conditions were obtained at power dissipations as low as $10 \mathrm{~W} / \mathrm{m}^{3}$. The advantages of the $\mathrm{OBC}$ as a novel device include its ability to decouple bubble generation and particle-bubble contacting, its even distribution of shear rate in the cell as well as the additional fluctuating fluid motion produced under oscillatory flow. Furthermore, the flotation kinetics in the cell were shown to be unaffected by slurry rheology indicating the cell is best suited to niche application in the processing of ore types exhibiting highly viscous non-Newtonian behaviour.

The application the $\mathrm{OBC}$ as a research tool for evaluating the effect of hydrodynamics on particle-bubble contacting has also achieved much success. The flotation kinetics in the $\mathrm{OBC}$ were shown to be dominated by the bulk fluid motion rather than the micro-turbulence as predicted by fundamental flotation models. This indicated that the underlying mechanisms of contacting are different to those derived for homogeneous isotropic turbulence. Thus, although the OBC could not be used for the direct validation of fundamental flotation models, it was able to highlight the importance of the shear rate due to bulk fluid motion in determining flotation behaviour. This aspect has yet to be considered in the current range of turbulent flotation models. Furthermore the results suggested that the linear dependence of the flotation kinetics on the gas hold-up, which is proposed by the fundamental models of flotation, is highly implausible since no such relationship was observed when gas hold-up was varied at constant bubble surface area flux. This suggests that the framework used in deriving fundamental flotation models may need to be adjusted to incorporate a flux based approach.

The OBC was therefore concluded to be an effective research tool which has provided specific insights into the role of hydrodynamics in promoting particlebubble contacting. The markedly different mode of power input has allowed the study of particle-bubble contacting in a unique hydrodynamic environment and has highlighted specific areas for the future development of flotation modelling. 


\section{Table of Contents}

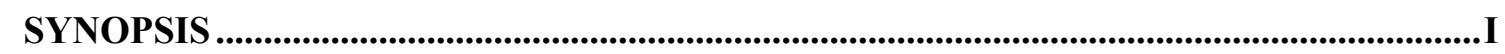

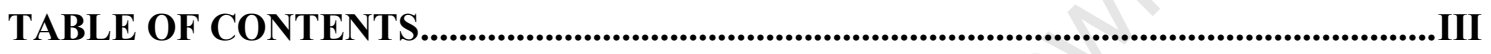

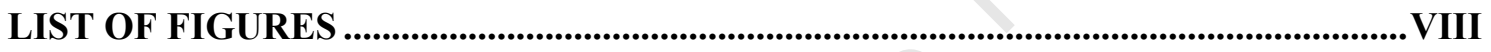

LIST OF TABLES ….............................................................................................................................. XII

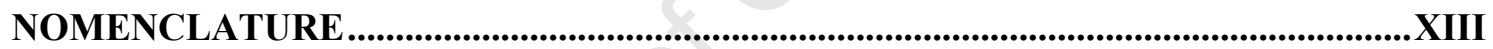

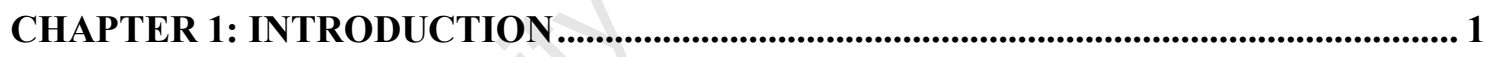

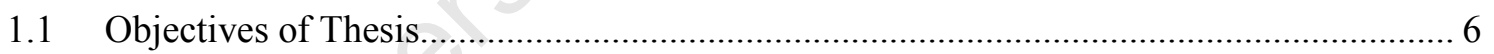

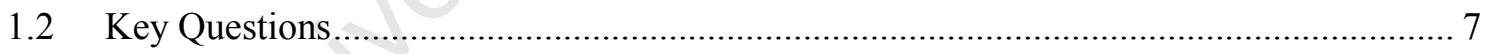

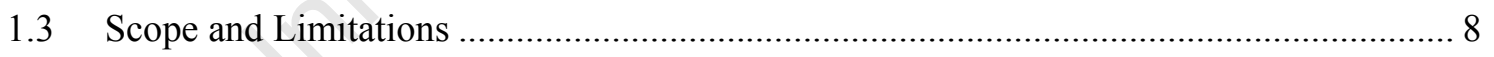

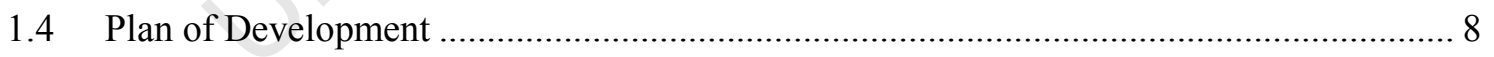

CHAPTER 2: LITERATURE REVIEW ............................................................................. 11

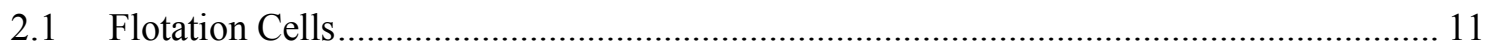

2.1.1 Mechanical Flotation Cells .......................................................................... 12

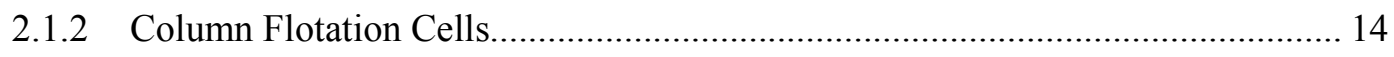

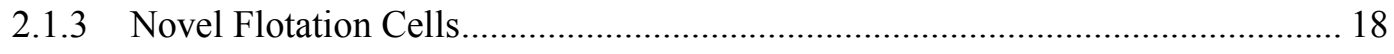

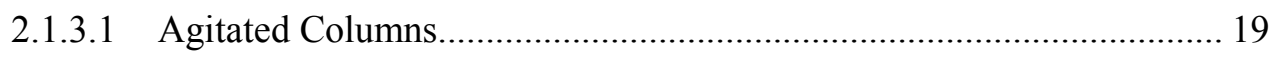

2.1.3.2 Reactor/Separator Type Cells.......................................................... 22 


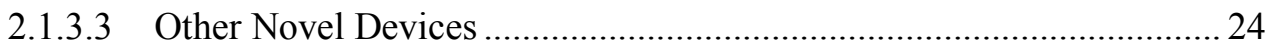

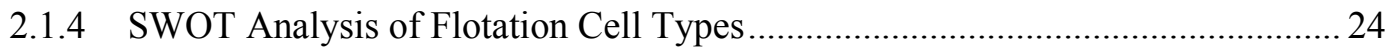

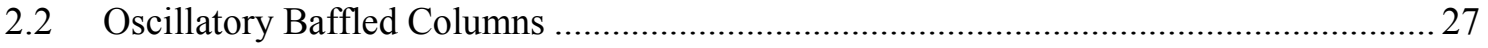

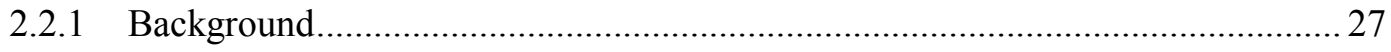

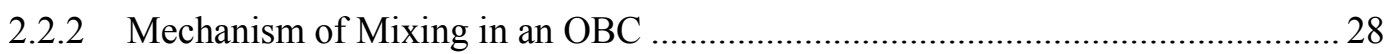

2.2.3 Characteristic Dimensions and Dimensionless Groups ........................................ 30

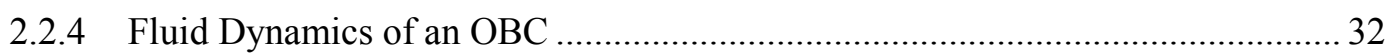

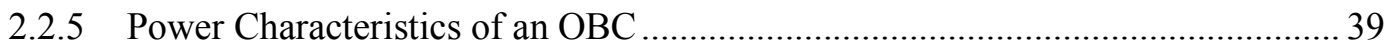

2.2.5.1 The Quasi-Steady State Model.............................................................. 42

2.2.5.2 The Acoustic Model ........................................................................... 44

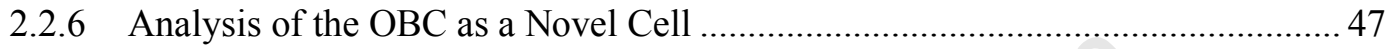

2.3 The Effect of Hydrodynamics on Particle-Bubble Contacting ......................................... 49

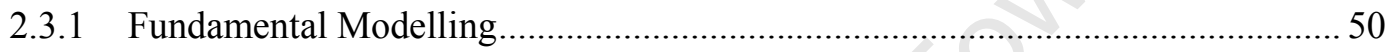

2.3.1.1 The Statistical Description of Turbulence............................................... 50

2.3.1.2 Particle-Bubble Collision under Turbulent Conditions..........................53

2.3.1.3 Particle-Bubble Collision/Interception Models ......................................... 67

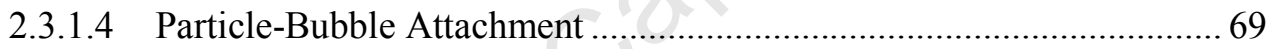

2.3.1.5 Particle-Bubble Detachment under Turbulence ................................... 70

2.3.2 Rheological Characteristics of Mineral Suspensions........................................... 74

2.3.2.1 Rheology Fundamentals ................................................................... 75

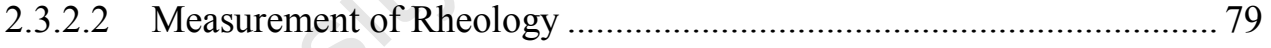

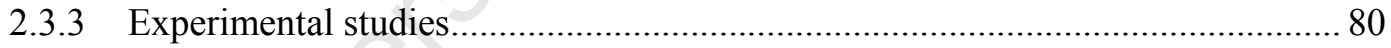

2.3.4 Summary of the Effect of Hydrodynamics on Particle-Bubble Contacting ......... 84

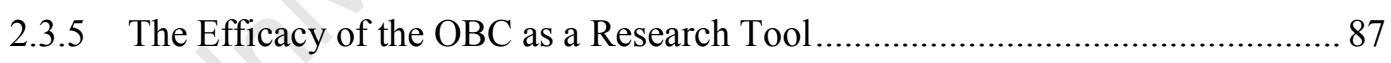

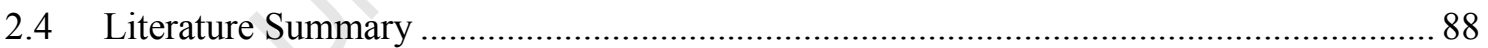

CHAPTER 3: MATERIALS AND METHODS.................................................................. 91

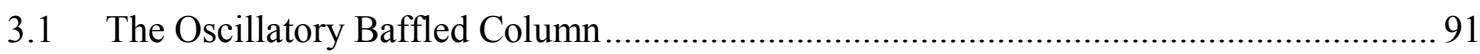

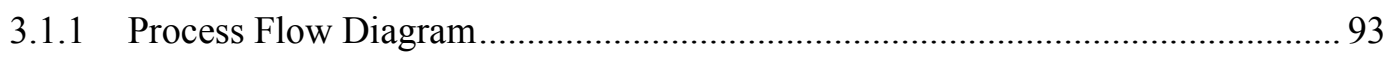

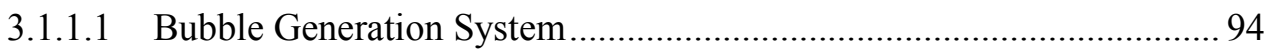

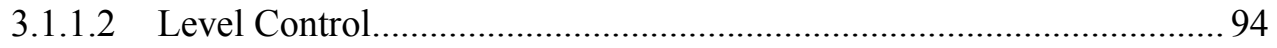

3.1.1.3 Pressure and Displacement Measurement ............................................. 95

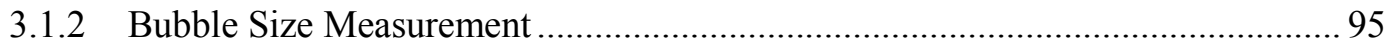

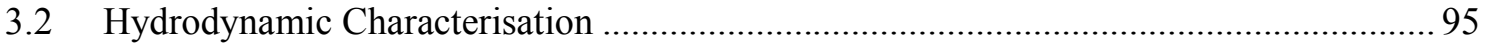




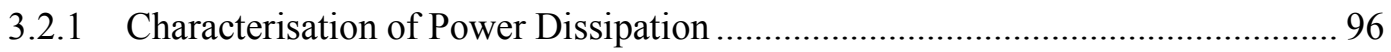

3.2.2 The Effect of Power Dissipation on Bubble Size ................................................. 97

3.2.3 The Effect of Operating Conditions on Gas Hold-Up ........................................... 99

3.2.4 The Effect of Operating Conditions on Mixing.................................................. 100

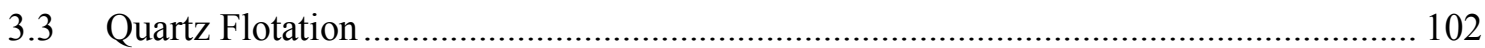

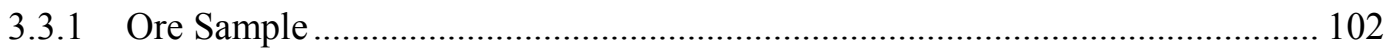

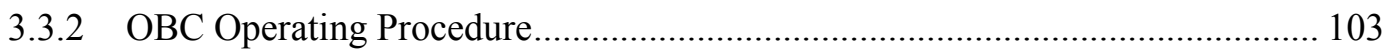

3.3.3 Experimental Programme _............................................................................. 104

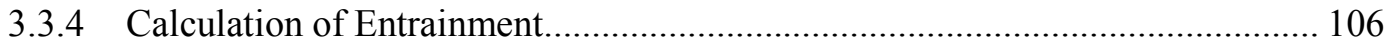

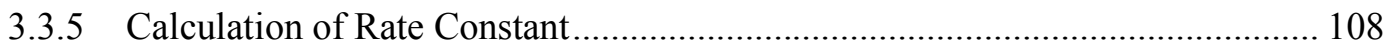

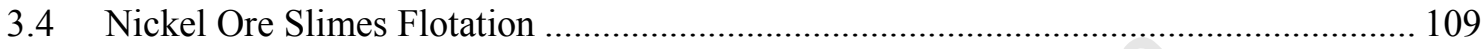

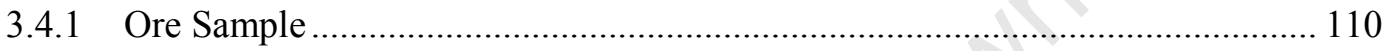

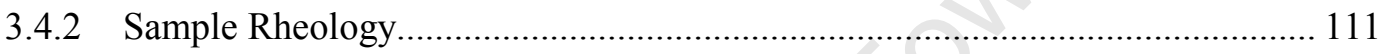

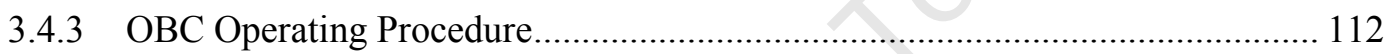

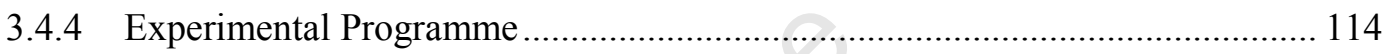

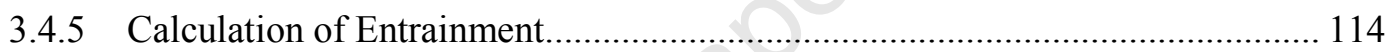

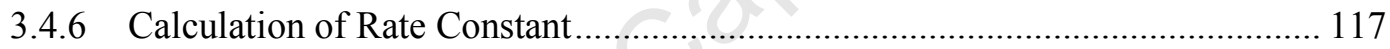

CHAPTER 4: HYDRODYNAMIC CHARACTERISATION............................................... 119

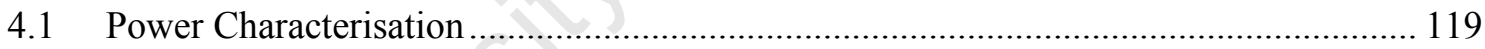

4.1.1 Ungassed Power Characteristics......................................................................... 120

4.1.1.1 Ratio of Inertial and Frictional Contributions ..................................... 121

4.1.1.2 Time-Averaged Power Dissipation .................................................... 123

4.1.1.3 Comparison with the Quasi-Steady State Model ................................ 124

4.1.1.4 Comparison with the Acoustic Model................................................. 125

4.1.2 Gassed Power Characteristics......................................................................... 128

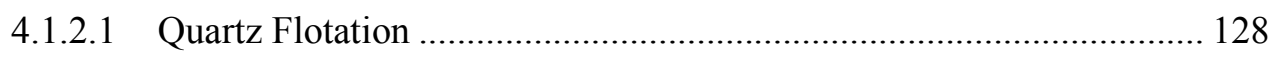

4.1.2.2 Nickel Ore Slimes Flotation .............................................................. 129

4.2 The Effect of Power Dissipation on Bubble Size ............................................................. 132

4.3 The Effect of Power Dissipation on Gas Hold-up ............................................................ 135

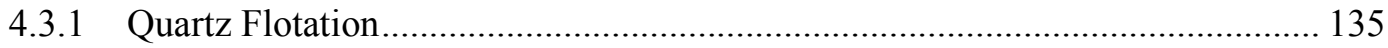

4.3.2 Nickel Ore Slimes Flotation ........................................................................... 137

4.4 The Effect of Power Dissipation on Mixing Characteristics ........................................ 138

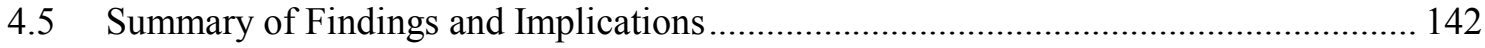


5.1 Flotation Response under Weakly Hydrophobic Conditions......................................... 145

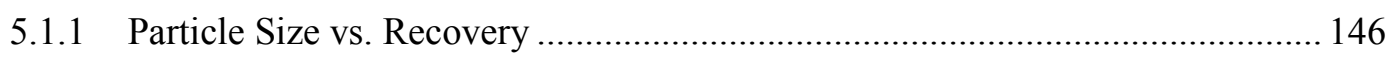

5.1.2 Behaviour of the Sub 3 Micron Size Fraction .................................................. 147

5.1.3 The Effect of Power Dissipation on Flotation Recovery ................................... 148

5.1.4 Summary of Findings and Actions Taken ...................................................... 152

5.2 Flotation Response under Moderately Hydrophobic Conditions .................................... 153

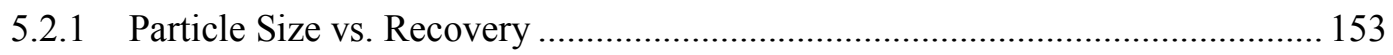

5.2.2 The Effect of Power Dissipation on the Flotation Recovery ……...................... 154

5.3 Rate Constants Obtained Using the Tanks-in-Series Model........................................... 157

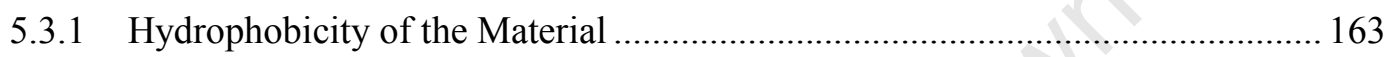

5.3.2 Non-Homogeneous Distribution of Shear Rate in Conventional Cells ............... 164

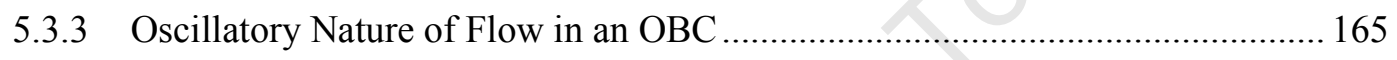

5.4 The Effect of Bubble Size \& Gas Hold-up on Flotation Kinetics..................................... 169

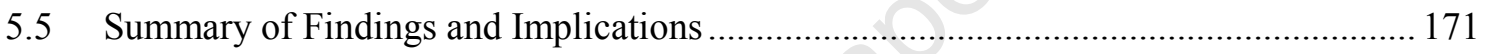

CHAPTER 6: NICKEL ORE SLIMES FLOTATION..................................................... 175

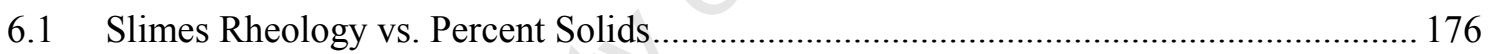

6.2 The Effect of Power Dissipation on the Rate of Flotation ............................................. 179

CHAPTER 7: SUMMARY \& CONCLUSIONS ................................................................. 187

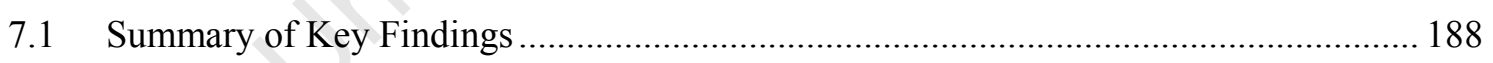

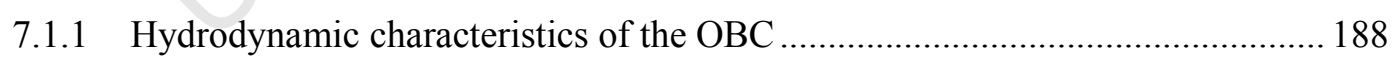

7.1.2 The Effect of Power Dissipation on Gas Phase Characteristics in the OBC ...... 189

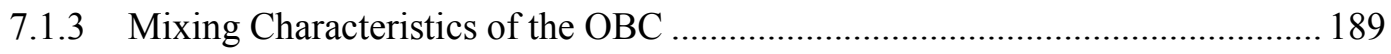

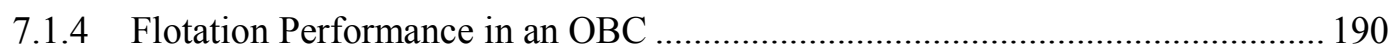

7.1.5 The Effect of Power Dissipation on the Flotation Rate ..................................... 190

7.1.6 Comparison of Flotation Trends with Previous Studies ..................................... 191

7.1.7 The Relationship between the Rate of Flotation and Slurry Rheology ............... 192

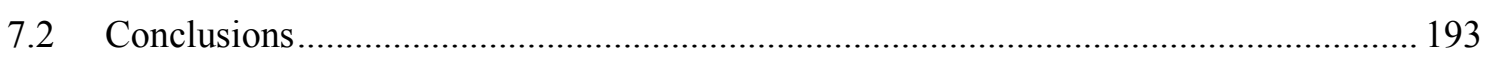

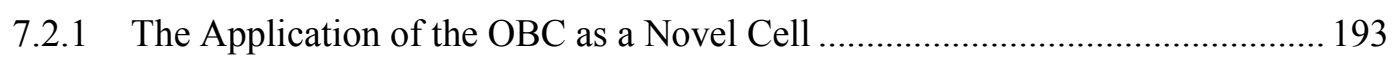

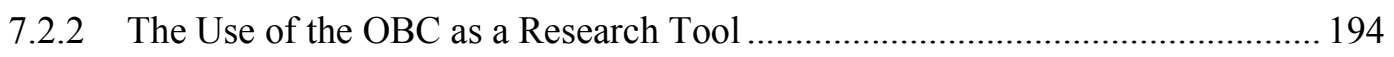


7.3 Recommendations for Future Work.

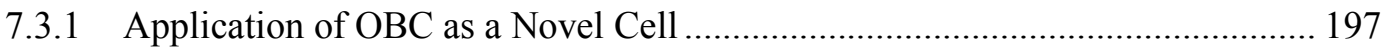

7.3.2 The Investigation of Hydrodynamics and Particle-Bubble Contacting .............. 198

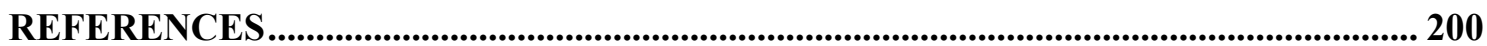

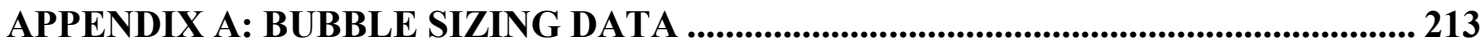

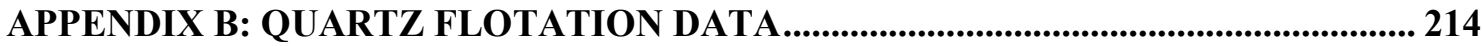

APPENDIX C: NICKEL ORE SLIMES FLOTATION ................................................... 219

APPENDIX D: DERIVATION OF EQUATION 2.26............................................................ 221 


\section{List of Figures}

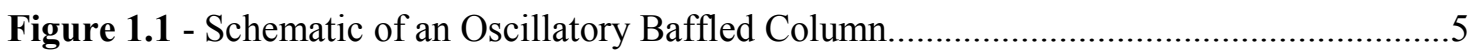

Figure 2.1 - Schematic of a Modern-Day Mechanical Cell (TankCell®, Outotec, 2008).......... 13

Figure 2.2 - Schematic of a Column Flotation Cell (McKay et. al., 1988) ................................ 15

Figure 2.3 - Schematic of the Static In-line Mixer Configuration Used in the MicroCell ${ }^{\mathrm{TM}}$.

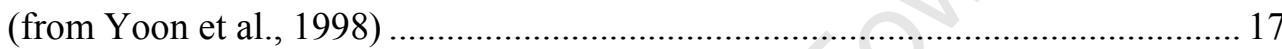

Figure 2.4 - Schematic of a Packed Flotation Column (from Rubinstein, 1995) ....................... 18

Figure 2.5 - Flotation Performance of an Agitated Column Cell relative to other Flotation

Cell Technologies as a Function of Particle Size (from Breytenbach, 1995)........ 20

Figure 2.6 - Flotation Performance of the Agitated Column as a Function of Specific Power Input for Flotation of $+106-150$ micron Quartz (from Breytenbach, 1995) and Flotation of Fine Pyrite (62\% passing 25 micron) (from Ityokumbul et al., 2000).......

Figure 2.7 - Schematic of a Jameson Flotation Cell (from Rubinstein, 1995)........................... 23

Figure 2.8 - Mixing Mechanism in an OBC (from Ni \& Jian, 2005) ......................................... 29

Figure 2.9 - Characteristic Dimensions of an Oscillatory Flow System .................................... 30

Figure 2.10 - Shear Rate Distribution within an OBC Oscillating at $5 \mathrm{~mm}$ and $2.5 \mathrm{~Hz}$, taken at the Point of Maximum Fluid Velocity $\left(\mathrm{Re}_{\mathrm{o}}=7850\right)($ Bakker, 2005).

Figure 2.11 - Shear Rate Distribution in a Bateman 120 litre Pilot Flotation Cell operating at

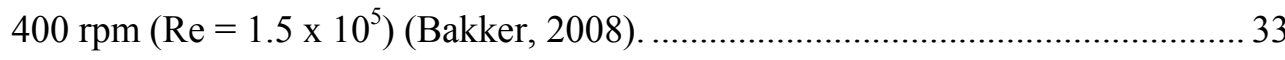

Figure 2.12 - Mechanism of Vortex Trapping in a Laminar Eddy (Tooby et al., 1977) ............ 36

Figure 2.13 - The Effect of Apparent Viscosity on the Ratio of Axial to Radial Velocities in an $\mathrm{OBC}$ for a Water-Glycerol and Water-CMC Mixture (Fitch et al., 2005)......... 38

Figure 2.14 - Schematic of the Components of Instantaneous Power Input to an OBC ............ 41

Figure 2.15 - Schematic Representation of a Turbulent Kinetic Energy Spectrum (from Deglon, 1998). 
Figure 2.16 - Stokes Number as a Function of Particle Diameter and Energy Dissipation Rate for Quartz Particles in Water $\left(\rho_{\mathrm{s}}=2700 \mathrm{~kg} / \mathrm{m}^{3}, \rho_{\mathrm{f}}=1000 \mathrm{~kg} / \mathrm{m}^{3}, \mu=1 \mathrm{cP}\right) . .58$

Figure 2.17 - Stokes Number as a Function of Bubble Diameter and Energy Dissipation Rate for Air Bubbles in Water $\left(\rho_{\mathrm{f}}=1000 \mathrm{~kg} / \mathrm{m}^{3}, \rho=1 \mathrm{cP}\right)$

Figure 2.18 - Model Predictions for the Collision Frequency of Two Particles of Equal Density as a Function of Stokes Number with One Particle having a Constant

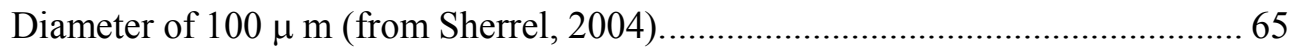

Figure 2.19 - Shear Stress vs. Shear Rate Curves for Different Fluids (Burdukova, 2007) ..... 75

Figure 2.20 - Apparent Viscosity of a Typical Pseudo Plastic Fluid compared with a Newtonian Fluid ..................................................................................... 78

Figure 2.21 - Schematic of a Typical Cup and Spindle Rheometer (Burdukova, 2007) .......... 79

Figure 3.1 - Schematic of the Oscillatory Baffled Column Flotation Cell................................. 92

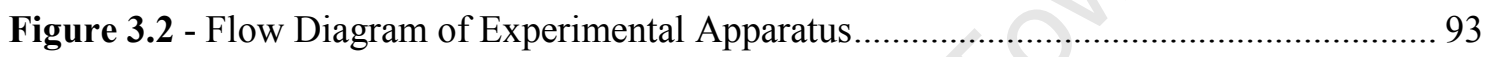

Figure 3.3 - Bubble Size Distribution Produced by the Static In-line Mixer............................. 99

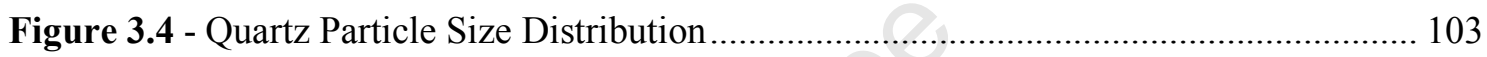

Figure 3.5 - Particle Entrainability as a Function of Particle Size (from Deglon, 1998) ......... 108

Figure 3.6 - Nickel Ore Slimes Particle Size Distribution ...................................................... 111

Figure 3.7 - Graph Illustrating the Difficulty in Estimating Entrainment at Low Water Recovery Using the Savassi Model.

Figure 3.8 - Graph Showing the Modelled Entrainment Mass Flow and the Total Mass Flow in the Concentrate Stream.

Figure 4.1 - Inertial and Frictional Components of the Overall Power Input for $\mathrm{x}_{\mathrm{o}}=5 \mathrm{~mm} \&$ $\mathrm{f}=2.5 \mathrm{~Hz}$

Figure 4.2 - Correlation between Ratio of Inertial to Frictional Forces in the Fluid and the Strouhal Number

Figure 4.3 - Time-Averaged Power Dissipation in the Agitated Zone of the OBC as a Function of Frequency and Amplitude.

Figure 4.4 - Comparison of Experimentally Determined Power Dissipation with the Predictions of the Quasi-Steady State Model.

Figure 4.5 - Correlation between Time-Averaged Power Dissipation and the Power Input Predicted by the Acoustic Model.

Figure 4.6 - Correlation Between Mixing Length and Strouhal Number. 127

Figure 4.7 - Parity Chart Comparing Power Dissipation for Ungassed Conditions and the Power Input during Quartz Flotation Tests 
Figure 4.8 - Parity Chart between the Power Measured under Gassed Conditions and the Power Correlation for Ungassed Power for Gas Hold-Up between $21.8 \%$ and $35.4 \%$

Figure 4.9 - Diagram Showing the Difference Between the Measured Pressure Signal and the Fitted Pressure Curve for the OBC Operating at $25 \mathrm{~mm}$ and $1.35 \mathrm{~Hz}$ with a Gas Hold-Up of $30.0 \%$

Figure 4.10 - The Effect of Power Dissipation on the Bubble Size in the OBC.

Figure 4.11 - The Relationship between Power Dissipation \& Gas Hold-up during Quartz Flotation Tests. (Open points denote $\mathrm{J}_{\mathrm{g}}=0.12 \mathrm{~cm} / \mathrm{s}$, Closed points denote $\mathrm{J}_{\mathrm{g}}=$ $0.21 \mathrm{~cm} / \mathrm{s})$ 136

Figure 4.12 - The Effect of Power Dissipation on Gas Hold-up during Nickel Ore Slimes Flotation Tests. $\left(\mathrm{J}_{\mathrm{g}}=0.64 \mathrm{~cm} / \mathrm{s}\right)$

Figure 4.13 - The Effect of Power Input on the Mixing Characteristics in the OBC relative to a Standard Column

Figure 4.14 - The Effect of the Number of Tanks-in-Series on the Recovery of a Cell with a Residence Time of 2.7 min for Different Values of Rate Constant.

Figure 4.15 - The Effect of the Number of Tanks-in-Series on the Overall Rate Constant Normalised Relative to the Rate Constant obtained at $\mathrm{N}=3$

Figure 5.1 - The Relationship between Particle Size and Recovery for the OBC Operating as a Standard Column Cell under Weakly Hydrophobic Conditions 146

Figure 5.2 - The Effect of Power Dissipation on the Recovery of Weakly Hydrophobic 3 - 17 micron Quartz Particles

Figure 5.3 - The Effect of Power Dissipation on the Recovery of Weakly Hydrophobic 17 - 36 micron Quartz Particles

Figure 5.4 - The Effect of Power Dissipation on the Recovery of Weakly Hydrophobic 36 - 76 micron Quartz Particles .

Figure 5.5 - The Effect of Power Dissipation on the Recovery of Weakly Hydrophobic 76 - 104 micron Quartz Particles ......

Figure 5.6 - Comparison of Recoveries Obtained in a Standard Column for the Weakly Hydrophobic and Moderately Hydrophobic Conditions with the Cell Residence Time and Air Flow Rate Substantially Reduced (c.f. Table 3.3).

Figure 5.7 - The Effect of Power Dissipation on the Recovery of Moderately Hydrophobic 3 - 17 micron Quartz Particles 
Figure 5.8 - The Effect of Power Dissipation on the Recovery of Moderately Hydrophobic 17 - 36 micron Quartz Particles

Figure 5.9 - The Effect of Power Dissipation on the Recovery of Moderately Hydrophobic 36 - 76 micron Quartz Particles.

Figure 5.10 - The Effect of Power Dissipation on the Recovery of Moderately Hydrophobic 76 - 104 micron Quartz Particles

Figure 5.11 - The Effect of Power Dissipation on the Flotation Rate Constant for Moderately Hydrophobic Quartz Particles at an Oscillation Amplitude of 10 $\mathrm{mm}$.

Figure 5.12 - Flotation Response Moderately Hydrophobic Quartz at an Amplitude of 5 mm using the Tanks-in-Series Model.

Figure 5.13 - Flotation Response Moderately Hydrophobic Quartz at an Amplitude of 10 $\mathrm{mm}$ using the Tanks-in-Series Model.

Figure 5.14 - Flotation Response Moderately Hydrophobic Quartz at an Amplitude of 25 mm using the Tanks-in-Series Model.

Figure 5.15 - Schematic of Fluctuating Velocity in an OBC and in a Conventional Cell 165

Figure 5.16 - Root Mean Squared Fluid Velocities Arising from Oscillatory Flow in the $\mathrm{OBC}$ as a Function of the Mean Power Input

Figure 5.17 - Normalised Rate Constant of the 17-36 micron Quartz Particles as a Function of the Root Mean Squared Baffle Cage Velocity 168

Figure 6.1 - Example of Rheogram obtained for Nickel Ore Slimes at High and Low Percent Solids

Figure 6.2 - The Effect of Solids Fraction on Yield Stress and Viscosity 178

Figure 6.3 - The Effect of Power Dissipation on the Flotation Rate Constant at $11.2 \%$ Solids

Figure 6.4 - The Effect of Power Dissipation on the Flotation Rate Constant at 4.6 \% Solids 180

Figure 6.5 - Comparison of rates constants obtained for nickel in MKO slimes flotation and 0-12 micron quartz particles in a quartz-amine system 182

Figure 6.6 - Comparison of Apparent Viscosities of the Different Slurries Tested 183 


\section{List of Tables}

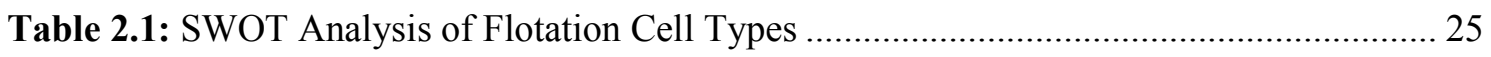

Table 2.2: Dimensionless Groups Describing Flow in an OBC (Ni \& Gough, 1997) ............... 30

Table 2.3: SWOT Analysis of the Application of an OBC as a Novel Flotation Cell

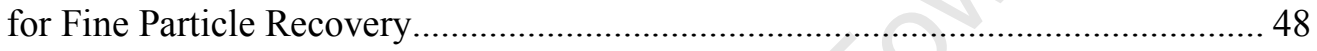

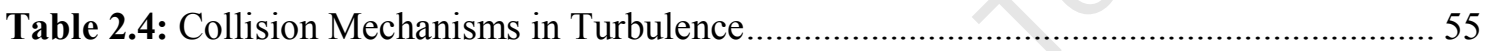

Table 2.5: Attachment and Detachment Forces on the Particle-Bubble Aggregate ................... 71

Table 2.6: Summary of Previous Experimental Studies on Hydrodynamics and Flotation Rate at Constant Bubble Size................................................................... 81

Table 3.1: Dimensionless Groups of OBC Design (Refer Table 2.2) ….................................. 92

Table 3.2: Experimental Conditions for Quartz Flotation Experiments................................... 106

Table 5.1: Relative Error in Recovery and Rate Constant per Particle Size Class.................... 154

Table A.1: Results of Bubble Size Analysis as function of Power Dissipation* ..................... 213

Table B.1: Weakly Hydrophobic Quartz Flotation Results................................................... 214

Table B.2: Moderately Hydrophobic Quartz Flotation Results for Column

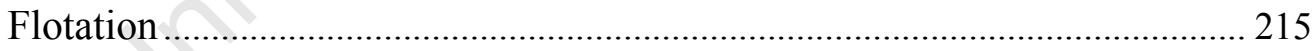

Table B.3: Moderately Hydrophobic Quartz Flotation Results for $5 \mathrm{~mm}$ Amplitude.

Table B.4: Moderately Hydrophobic Quartz Flotation Results for $10 \mathrm{~mm}$ Amplitude.

Table B.5: Moderately Hydrophobic Quartz Flotation Results for $25 \mathrm{~mm}$ Amplitude. 218

Table C.1: Normalised High Rheology Flotation Results 219

Table C.1: Normalised Low Rheology Flotation Results. 220 


\section{Nomenclature}

$a$

A

b

Bo'

$\mathrm{C}_{\mathrm{c}, \mathrm{i}}$

$\mathrm{C}_{\mathrm{i}}$

$\mathrm{C}_{\mathrm{o}}$

$\mathrm{C}(\mathrm{t})$

$d_{b}$

$d_{p}$

$\mathrm{D}$

$\mathrm{D}_{\mathrm{o}}$

$\mathrm{E}_{\mathrm{B}}$

$\mathrm{E}_{\mathrm{K}}$

$\mathrm{E}(\mathrm{t})$

$\mathrm{ENT}_{\mathrm{i}}$

f

$\mathrm{F}_{\mathrm{A}}$

$\mathrm{g}$

$\mathrm{G}_{\mathrm{fr}}$

h

J

$\mathrm{J}_{\mathrm{g}}$

$\mathrm{k}$

$\mathrm{K}_{\mathrm{i}}$
Acceleration $\left(\mathrm{m} / \mathrm{s}^{2}\right)$

Cross sectional area $\left(\mathrm{m}^{2}\right)$

Added mass coefficient (dimensionless)

Bond number (dimensionless)

Cunningham slip correction factor

Mass concentration of size class i $(\mathrm{g} / \mathrm{L})$

Drag coefficient of an orifice baffle

Measured conductivity signal (mV)

Bubble diameter (m)

Particle diameter (m)

Column diameter (m)

Orifice diameter $(\mathrm{m})$

Energy barrier ( $\mathrm{J})$

Kinetic energy $(\mathrm{J})$

Exit age distribution (dimensionless)

Entrainment fraction of size class $\mathrm{i}$

Frequency $(\mathrm{Hz})$

Force of adhesion (N)

Gravitational acceleration $\left(\mathrm{m} / \mathrm{s}^{2}\right)$

Gas flow rate $\left(\mathrm{m}^{3} / \mathrm{s}\right)$

Height (m)

Power (W)

Superficial gas velocity $(\mathrm{m} / \mathrm{s})$

Flotation rate constant $\left(\mathrm{min}^{-1}\right)$

Fitted parameter for fluid inertia 


\begin{tabular}{|c|c|}
\hline 1 & Mixing length (m) \\
\hline $\mathrm{L}$ & Column length (m) \\
\hline$m_{i}$ & Mass of size class i (g) \\
\hline $\mathrm{N}$ & Number of tanks-in-series \\
\hline $\mathrm{N}_{\mathrm{b}}$ & Number of bubbles per unit volume $\left(\mathrm{m}^{-3}\right)$ \\
\hline $\mathrm{N}_{\mathrm{p}}$ & Number of particles per unit volume $\left(\mathrm{m}^{-3}\right)$ \\
\hline $\mathrm{P}$ & Pressure $(\mathrm{Pa})$ \\
\hline $\mathrm{P}_{\mathrm{a}}$ & Probability of attachment \\
\hline $\mathrm{P}_{\mathrm{c}}$ & Probability of collision / interception \\
\hline$P_{d}$ & Probability of detachment \\
\hline $\mathrm{r}_{\mathrm{b}}$ & Bubble radius (m) \\
\hline $\mathrm{r}_{\mathrm{e}}$ & Eddy radius (m) \\
\hline $\mathrm{r}_{\mathrm{p}}$ & Particle radius (m) \\
\hline $\mathrm{R}_{\mathrm{i}}$ & Recovery of size class i \\
\hline $\mathrm{R}_{\mathrm{imp}}$ & Radius of impeller (m) \\
\hline $\mathrm{R}_{\mathrm{w}}$ & Water recovery \\
\hline $\operatorname{Re}_{\mathrm{n}}$ & Nett flow Reyolds number (dimensionless) \\
\hline $\mathrm{Re}_{\mathrm{o}}$ & Oscillatory Reyolds number (dimensionless) \\
\hline $\mathrm{S}$ & Fractional baffle free area \\
\hline $\mathrm{Sb}$ & Bubble surface area flux $\left(\mathrm{m}^{2} / \mathrm{m}^{2} . \mathrm{s}\right)$ \\
\hline $\mathrm{St}$ & Strouhal number (dimensionless) \\
\hline$t_{i}$ & Induction time (s) \\
\hline $\mathrm{T}$ & Absolute temperature $(\mathrm{K})$ \\
\hline $\mathrm{T}_{\mathrm{L}}$ & Lagrangian time integral scale (s) \\
\hline TKE & Turbulent Kinetic Energy $(\mathrm{J} / \mathrm{kg})$ \\
\hline $\mathrm{u}$ & Velocity (m/s) \\
\hline $\mathrm{U}$ & Velocity (m/s) \\
\hline$U^{\prime}$ & Turbulent fluctuating velocity $(\mathrm{m} / \mathrm{s})$ \\
\hline $\mathrm{U}_{\mathrm{b}}$ & Bubble rise velocity $(\mathrm{cm} / \mathrm{s})$ \\
\hline $\mathrm{U}_{\mathrm{osc}}$ & Oscillatory velocity of the fluid $(\mathrm{m} / \mathrm{s})$ \\
\hline $\mathrm{V}_{\text {cage }}$ & Baffle Cage Velocity (m/s) \\
\hline
\end{tabular}




$\begin{array}{ll}\mathrm{V}_{\text {cell }} & \text { Cell volume }\left(\mathrm{m}^{3}\right) \\ \mathrm{w}_{\text {accel }} & \text { Relative velocity between colliding particles }(\mathrm{m} / \mathrm{s}) \\ \mathrm{w}_{\text {conc }} & \text { Mass of water in the concentrate }(\mathrm{g}) \\ \mathrm{w}_{\text {feed }} & \text { Mass of water in the feed }(\mathrm{g}) \\ \mathrm{w}_{\text {shear }} & \text { Relative velocity between colliding particles }(\mathrm{m} / \mathrm{s}) \\ \mathrm{w}_{\text {tail }} & \text { Mass of water in the tail }(\mathrm{g}) \\ \mathrm{W}_{\mathrm{A}} & \text { Work of adhesion }(\mathrm{J}) \\ \mathrm{x}_{\mathrm{e}} & \text { Entrainment fraction } \\ \mathrm{x}_{\mathrm{o}} & \text { Amplitude of oscillation }(\mathrm{m}) \\ \mathrm{z}_{\mathrm{o}} & \text { Height of liquid above the contact area }(\mathrm{m}) \\ \mathrm{Z} & \text { Length of agitated zone }(\mathrm{m}) \\ \mathrm{Z}_{\mathrm{ij}} & \text { Volumetric rate of collision between } \mathrm{i} \text { and } \mathrm{j}\left(\mathrm{m}^{-3} \mathrm{~s}^{-1}\right) \\ \mathrm{Z}_{\mathrm{pb}} & \text { Volumetric rate of collision between particles and bubbles }\left(\mathrm{m}^{-3} \mathrm{~s}^{-1}\right) \\ \mathrm{Z}_{\mathrm{pb}}^{\prime} & \text { Volumetric rate of detachment }\left(\mathrm{m}^{-3} \mathrm{~s}^{-1}\right)\end{array}$

\section{Greek Letters}

$\begin{array}{ll}\dot{\gamma} & \text { Shear rate }\left(\mathrm{s}^{-1}\right) \\ \Gamma(\mathrm{N}) & \text { Gamma function } \\ \delta & \text { Phase lag between start of measurement and start of cycle (rad) } \\ \varepsilon & \text { Energy dissipation rate }(\mathrm{W} / \mathrm{kg}) \\ \eta & \text { Kolmogorov microscale of turbulence }(\mathrm{m}) \\ \theta & \text { Contact angle (degrees) } \\ \theta_{\mathrm{t}} & \text { Angle of tangency }(\mathrm{rad}) \\ \kappa_{\mathrm{B}} & \text { Boltzmann constant } \\ \mu & \text { Visocity }(\mathrm{Pa} . \mathrm{s}) \\ \mu_{\text {app }} & \text { Apparent viscosity }(\mathrm{Pa} . \mathrm{s}) \\ \rho & \text { Density }\left(\mathrm{kg} / \mathrm{m}^{3}\right) \\ \sigma & \text { Surface tension }(\mathrm{N} / \mathrm{m}) \\ \tau & \text { Residence time }(\mathrm{s})\end{array}$




\section{Subscripts}

$$
a
$$$$
\mathrm{b}
$$$$
\text { f }
$$$$
\mathrm{p}
$$$$
\text { i }
$$$$
\text { j }
$$

true
Bubble relaxation time (s)

Gas residence time (s)

Particle/bubble relaxation time (s)

Kolmogorov timescale

Particle relaxation time (s)

Slurry yield stress $(\mathrm{Pa})$

Kinematic viscosity $\left(\mathrm{m}^{2} / \mathrm{s}\right)$

Turbulent eddy viscosity $\left(\mathrm{m}^{2} / \mathrm{s}\right)$

Gas hold-up (\%)

Angle of capillary force (rad)

Angluar frequency ( $\mathrm{rad} / \mathrm{s})$

\section{Aggregate}

Bubble

Friction / Fluid

Particle

Inertia / particle class

Particle or bubble class

True flotation 


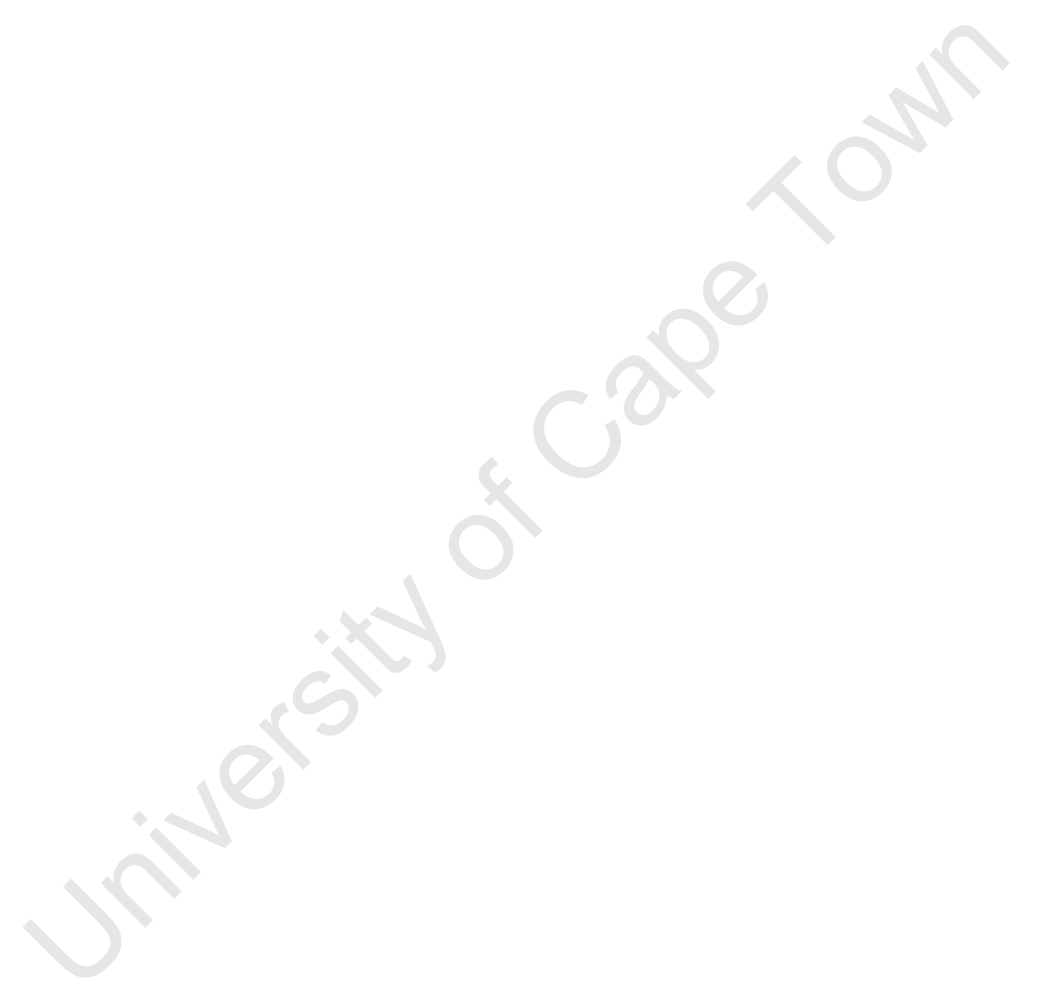




\section{Chapter 1}

\section{Introduction}

On its introduction to the minerals processing industry in 1905, froth flotation was heralded as one of the most significant technological developments of its time (Fuerstenau, 1999). The process was found to be a highly effective method of upgrading lower grade ores prior to smelting and leaching and therefore allowed the exploitation of a wide range of new lower grade ore bodies. Since then, flotation has become one of the most widely used mineral beneficiation techniques and is responsible for a large proportion of global metal production today.

While this technology has been highly effective over the past 100 years, it increasingly faces a new set of challenges. As the global supply of high grade, easily floatable ore bodies starts to dwindle, mining companies are increasingly being forced to turn to low grade, finely disseminated ore bodies, which are far more difficult to recover using conventional flotation technology. While much focus has been given to the development of ultra-fine grinding techniques which are able to liberate these minerals prior to flotation, it is still unclear whether conventional flotation technologies will be able to adequately cope with the fine grind sizes produced. In addition, as particle size decreases, the rheology of the slurry often becomes more complex, particularly in ores which contain fibrous or platy gangue material (Tangsathitkulchai \& Austin, 1988, Chang \& Powell, 1994). These rheological complexities can be detrimental to flotation 
cell hydrodynamics in stirred systems (Moore \& Cossor, 1995, Fangary et. al., 2000, Arratia et. al., 2006).

Although improved flotation chemistry will almost certainly aid the treatment of fine mineral slurries, it is believed that the greatest improvements will come from optimising the hydrodynamic environment in which flotation takes place. One possible solution is the development of a specialized flotation machine in which the hydrodynamic environment can be more effectively optimised for the flotation of fine particles. It is hypothesised that the development of such a machine will also facilitate a greater understanding of the effect of hydrodynamics on the flotation process, thereby informing the further optimisation of current flotation technologies.

The aim of this thesis is to address these issues by the introduction of a novel flotation cell, which is able to decouple and independently optimise the various sub-processes occurring in flotation. This type of system provides a more tightly controlled hydrodynamic environment in which flotation can take place, thus allowing the optimisation of hydrodynamics for fine particle flotation. The cell also produces an even distribution of shear rate in the fluid. This means that particles are exposed to a narrower range of shear rates, which can be more effectively optimised throughout the cell volume. This ability is also believed to enable the cell to effectively treat rheologically complex material, by enabling the slurry yield stress to be overcome at all points in the fluid. It is envisaged that such a design will therefore significantly improve the flotation rate of fine particles relative to current cell technologies and will also provide a unique environment in which to investigate the role of hydrodynamics in promoting particle-bubble contacting.

Over the last 50 years, several new flotation machine designs have been proposed. Historically, the industry has been slow to adopt these technologies, given the high costs of development and the risks of replacing current technologies. For this reason, the overwhelming majority of flotation worldwide still takes place in mechanically agitated or conventional cells. While the design of these cells has improved significantly over time, the basic cell design remains largely the same. Conventional cells consist of large 
tanks which are vigorously agitated by a central impeller. This impeller generally provides power inputs of between 1 and $3 \mathrm{~kW} / \mathrm{m}^{3}$, although power inputs of up to 12 $\mathrm{kW} / \mathrm{m}^{3}$ have been used in some fine particle applications. The power input provided by this impeller is responsible for three important sub-processes, which are essential for successful particle collection, viz.:

Solid Suspension: Solid particles must be suspended in the fluid.

Gas Dispersion: Air must be sheared into bubbles and dispersed across the cell.

Particle-Bubble Contacting: Particles must then collide with these bubbles with sufficient energy to overcome the energy barrier between them and attach to the surface. Once attached, the particle must be transported to the top of the flotation cell for removal without becoming detached by turbulent eddies in the fluid.

It is believed that the recovery of fine particles in conventional cells could be significantly improved if these three sub-processes could be optimised with respect to the power input to the fluid. Although the role which power input plays in solid suspension and gas dispersion can be relatively easily investigated and optimised in conventional flotation cells, the role which power input plays in promoting particlebubble contacting is considerably more difficult to quantify, since particle-bubble contacting is affected not only by the distribution of power dissipation in the cell but also by the bubble size and solids suspension characteristics. This interdependency makes independent investigation and optimisation of particle-bubble contacting extremely difficult.

To date, most studies have dealt with this dilemma by installing frits or spargers in laboratory scale Rushton turbine cells in order to generate bubbles independently of the level of agitation in the cell. While much useful information has been gained using this methodology, the studies are still limited by the substantial difference in power dissipation between the impeller zone and the bulk fluid of the cell. This disparity of power dissipation in stirred systems makes it difficult to attribute the observed flotation kinetics to turbulence in the impeller zone or turbulence in the bulk fluid. This makes extrapolation of the results to larger cells extremely difficult. 
As a result, most flotation cells are principally designed to meet criteria for effective gas dispersion and solid suspension (Arbiter, 1999, Weber et. al., 1999, Deglon, 2005). In terms of particle-bubble contacting, it is widely believed that fine particles are largely insusceptible to detachment and will therefore respond well to higher power inputs. However, the extent to which power inputs can be increased remains an open discussion since the relationship between power input and particle-bubble contacting remains unclear.

Some studies have proposed alternative designs in which the sub-processes of solid suspension, gas dispersion and particle-bubble contacting are more effectively decoupled, allowing independent optimisation of each process. One such design proposed in the literature was the agitated column (Breytenbach, 1995, Ityokumbul et. al., 2000), which consisted of a standard flotation column, agitated by a series of impellers down its length. Results indicated that the design was able to significantly improve fine particle recovery relative to both a column and batch flotation cell at comparable concentrate grades. Despite the potential which the technology showed, the design was never trialled industrially.

This study aims to build on these studies by the introduction of a similar, yet novel flotation device, the Oscillatory Baffled Column (OBC). It is proposed that this type of device allows the decoupling of bubble generation from particle-bubble contacting and thus allows the power dissipation in the cell to be optimised for fine particle recovery.

The major benefit of this type of device is its significantly different agitation mechanism. The OBC consists of a standard column which is fitted with a series of evenly spaced orifice baffles mounted perpendicular to the direction of flow. A sinusoidal motion is then superimposed on the nett flow by either pulsing the fluid through the baffles, using a piston or bellows, or by oscillating the baffles themselves using a piston and cam mounted above the column. A schematic of the system is shown in Figure 1.1: 


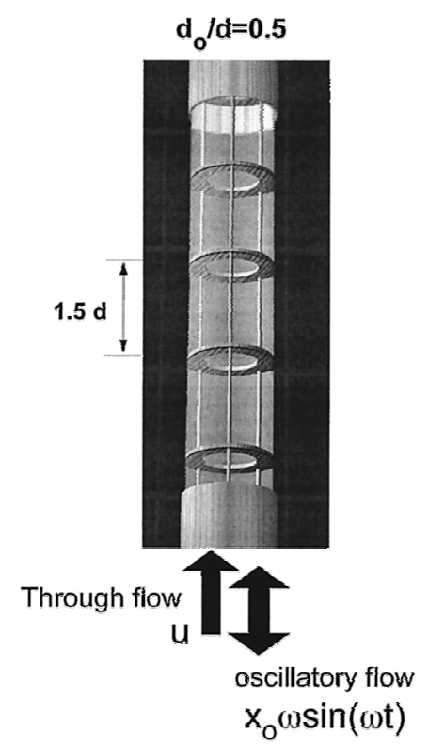

Figure 1.1 - Schematic of an Oscillatory Baffled Column (Harvey et al., 2001)

This design has been successfully applied to numerous industrial processes, particularly where performance is highly dependent on the shear rate in the fluid. These include biofermentation (Ni et. al., 1995, Gaidhani et. al., 2003), flocculation (Gao et. al., 1998), suspension polymerisation ( $\mathrm{Ni}$ et. al., 1999), protein refolding (Lee et. al., 2002), photocatalytic oxidation (Fabiyi \& Skelton, 1999, Gao et. al., 2003) and droplet breakage (Mignard et. al., 2003). This is because this type of agitation is able to produce a far more uniform shear rate distribution in the fluid than in a conventional stirred system, and allows the hydrodynamic environment to be tightly controlled since one is able to manipulate both the amplitude and frequency of power input to the cell.

It is proposed that these attributes, together with the cell's ability to decouple bubble generation and particle-bubble contacting, will allow the optimisation of the hydrodynamic environment for fine particle flotation, thereby substantially improving fine particle recoveries. The unique agitation mechanism and even distribution of shear in the cell is also believed to allow it to effectively treat rheologically complex mineral slurries which can be problematic in conventional stirred systems. 
In addition, the hydrodynamic environment in the $\mathrm{OBC}$ is distinctly different from that in a conventional system in that agitation is achieved by oscillating the fluid rather than stirring. This gives rise to a number of interesting research opportunities in terms of investigating the role which hydrodynamics play in promoting particle-bubble contacting. To date, most fundamental models of flotation indicate that particle-bubble contacting is controlled by the micro-turbulence in the fluid rather than the agitation mechanism directly. The nature of this micro-turbulent environment is governed by both the energy dissipation rate in the fluid and fluid viscosity. If this modelling premise is correct, one would expect similar flotation behaviour in different hydrodynamic environments, provided the micro-turbulent environment in each system was the same. Given the OBC's distinctly different agitation mechanism, it therefore provides a unique opportunity to evaluate this modelling premise and compare the trends obtained with the results of previous studies in stirred systems. It is believed that a comparison of this nature would provide specific insights into the effect of hydrodynamics on particlebubble contacting and thereby inform the further development of fundamental flotation models.

On the whole, the development of the $\mathrm{OBC}$ as a flotation cell has two major benefits to the current body of literature. Firstly, it provides a new and unique system for the improvement of fine particle flotation by decoupling and optimising the hydrodynamic environment in which flotation takes place. Furthermore, it is hypothesised that the unique attributes of the cell allow it to be used as a research tool to further enhance our understanding of the effect of hydrodynamics on particle-bubble contacting.

\subsection{Objectives of Thesis}

Given the current pressures on the flotation industry, it is proposed that a need exists for the development of a new flotation device which can effectively treat both fine material and rheologically complex ores slurries. It is believed that the development of such a device will also enhance our understanding of the role of the hydrodynamic environment in promoting particle-bubble contacting, and thus aid in the long term 
optimisation of existing flotation technologies. The objectives of this thesis are therefore twofold:

1. To evaluate the use of an Oscillatory Baffled Column as a novel flotation device for fine particle recovery as well as the recovery of particles in rheologically complex mineral slurries.

2. To evaluate the efficacy of the Oscillatory Baffled Column as a research tool for the investigation of the effect of the hydrodynamic environment on particle-bubble contacting.

In achieving these objectives, emphasis is placed on the comparison of the results obtained with those obtained in similar studies in stirred tank environments. This comparison provides insights into the role of hydrodynamics in promoting particlebubble contacting in different contacting environments. Furthermore, the flotation performance of the $\mathrm{OBC}$ is evaluated as a function of power dissipation at different slurry rheologies. This is because theory suggests that the combination of these two factors determines the micro-turbulent environment in the cell, as characterised by the size and intensity of the inertial and viscous dissipation sub-range eddies. This analysis allows an evaluation of the relative importance of fluid properties in determining the flotation response as a function of power input.

\subsection{Key Questions}

In order to achieve these objectives, the following key questions are posed:

1. What are the hydrodynamic characteristics of the OBC? How do they differ from conventional stirred systems? How well does the OBC approximate the conditions for which fundamental flotation models are developed?

2. How do the hydrodynamic characteristics of the $\mathrm{OBC}$ affect other flotation variables such as bubble size, gas hold-up and mixing in the cell? 
3. What is the effect of increasing the power dissipation on the particle-bubble contacting rate in the $\mathrm{OBC}$ ? How does it change as a function of particle size and hydrophobicity? How is this trend affected by changes in fluid properties such as rheology?

4. Is the OBC effective in the flotation of fine particles? Can this effectiveness be extended to systems exhibiting complex rheology?

5. To what extent are the particle-bubble contacting trends observed in the $\mathrm{OBC}$ consistent with those proposed by fundamental flotation models? Does the OBC provide additional insights into the nature of bubble-particle interactions which are not accounted for in the current models?

\subsection{Scope and Limitations}

The focus of this study is the use of the OBC as a novel hydrodynamic environment for enhanced particle-bubble contacting. The study will therefore exclude the effect of other factors such as pulp chemistry or froth effects on flotation rate since these factors can be effectively investigated in conventional flotation systems.

Furthermore, this study does not aim to develop new fundamental flotation models to explain the observed flotation responses. Rather, it aims to assess the extent to which the current fundamental flotation models are able to account for observed trends, and thereby highlight possible areas for future development.

\subsection{Plan of Development}

In order to achieve these objectives and evaluate these hypotheses, this thesis will first provide a review of the current range of flotation cell technologies. Specific areas for development are highlighted so as to contextualize the study. The existing body of knowledge on Oscillatory Baffled Columns is then reviewed with specific emphasis on the hydrodynamic and power characteristics of the cell. The existing body of knowledge on the effect of hydrodynamics on particle-bubble contacting is then reviewed from both a theoretical and experimental point of view. The trends predicted by theory and 
those obtained in practice are highlighted so as to provide a basis for the comparison of these trends with flotation data obtained in the OBC.

The experimental apparatus and methods are then described in Chapter 3 before Chapters 4, 5 and 6 report the findings of the experiments conducted and their implications. The OBC is first evaluated in terms of its power characteristics before the effect of operating conditions on flotation variables such as bubble size, gas hold-up and mixing are evaluated. The flotation performance of the cell is then evaluated using a quartz-amine slurry in order to benchmark the study against previous studies in stirred systems. The flotation trends are then discussed in terms of the expected trends from fundamental flotation models and compared with those obtained in previous studies.

The study is then extended to the flotation of a fine nickel ore slurry which is difficult to treat using conventional flotation technology due to its rheological complexity. This section aims to test the OBC's potential for engineering application as well as assess the effect of varying the micro-turbulent environment by varying the slurry rheology. The comparison of these results with the predictions of fundamental flotation models provides specific insights into the possible mechanisms underlying the flotation response.

The thesis then concludes by summarising the key findings of the study and their implications for flotation modelling, cell design and the application of $\mathrm{OBC}$ technology. Recommendations for future work are also made. 


\section{Chapter 2}

\section{Literature Review}

The following chapter reviews the literature relevant to this study. The current range of flotation cell technologies is reviewed and key areas for the development of novel flotation cell designs are identified. The characteristics and successes of Oscillatory Baffled Columns are then presented with specific emphasis on the fluid dynamic and power characteristics of the cells. The potential of these devices to improve fine particle recovery in flotation is then assessed. The body of knowledge on the effect of hydrodynamic parameters such as energy and rheology on particle-bubble contacting is then reviewed and the range of turbulent collision and detachment models reviewed. The chapter then reviews the various experimental studies on energy and particlebubble contacting which have been conducted in stirred systems. This provides a basis for comparison with the flotation trends obtained in the OBC in Chapters 5 and 6. The chapter then ends by assessing the OBC's potential as a research tool for investigating the effect of hydrodynamics on particle-bubble contacting.

\subsection{Flotation Cells}

Since the inception of froth flotation in 1905, numerous flotation machine designs have been proposed, particularly in the last 50 years. Traditionally, flotation has been performed in mechanically agitated tanks and the minerals processing industry has been 
particularly slow in its uptake of new cell technologies. The primary reason for this is that the development and testing of new flotation cell designs is costly and inherently risky to any mining company. Plant trials of large prototype cells are often costly, both in installation costs and in the potentially large losses of valuable mineral during testing. Despite this, many new flotation technologies have found increasing application in industry. The following section presents a brief overview of the major cell types; mechanical flotation cells, column flotation cells and novel flotation cells.

\subsubsection{Mechanical Flotation Cells}

Mechanical or "conventional" flotation cells are undoubtedly the industry standard for flotation. They consist of rectangular or rounded tanks which are vigorously agitated by a large impeller which is responsible for suspending solids and dispersing gas through the slurry. Many different cell designs have been used over the years but the most notable trend in design has been in the cell size. Conventional cell size has increased exponentially, particularly since the advent of rounded tank cells in the late 1980's. Flotation cells have increased from just $1 \mathrm{~m}^{3}$ in 1905 to up $300 \mathrm{~m}^{3}$ in present day applications (Fuerstenau, 1999). The driver for this trend is largely economic, with larger cells providing greater capacity at lower capital and maintenance costs. The relationship between cell size and metallurgical performance is still not well established, especially with respect to very large cells. However there is some evidence that cell size can be increased without the loss of metallurgical performance in some applications (Yianatosa et. al., 2007).

The impeller or rotor of the flotation cell generally provides power inputs of between 1 and $3 \mathrm{~kW} / \mathrm{m}^{3}$, although energy levels of up to $12 \mathrm{~kW} / \mathrm{m}^{3}$ have been used in some fine particle applications. A schematic of a modern mechanical flotation cell is shown in Figure 2.1: 


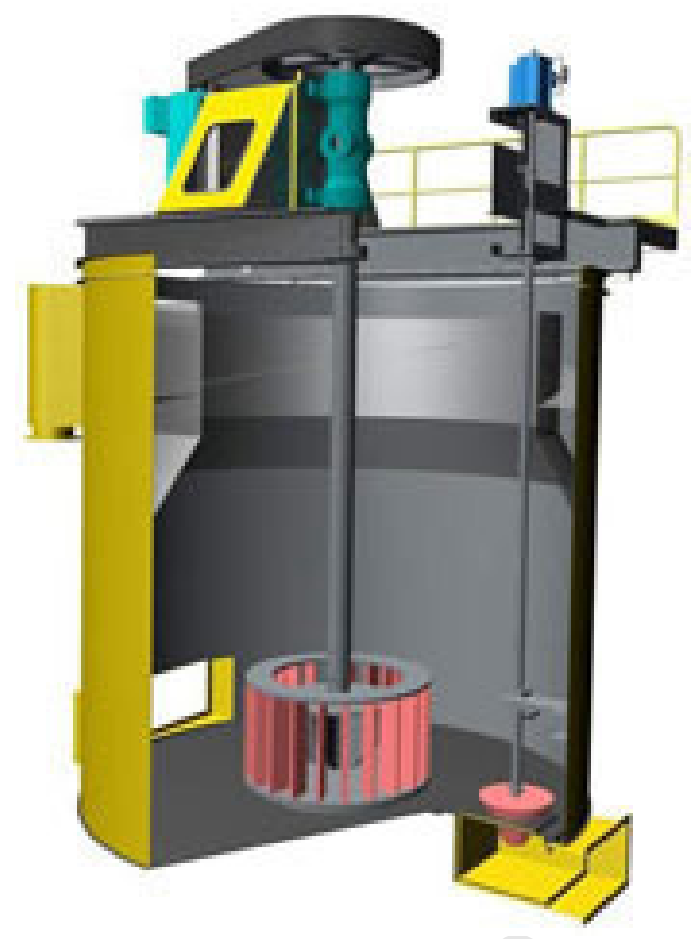

Figure 2.1 - Schematic of a Modern-Day Mechanical Cell (TankCell囚, Outotec, 2008)

Advantages of mechanical flotation cells include capacity and reliability. However, the processes of solid suspension, gas dispersion and particle-bubble contacting in this cell are inextricably interdependent and therefore difficult to optimise. In addition a large disparity of energy levels exists in the fluid. Power dissipation is concentrated around the impeller with low dissipation rates in the bulk of the fluid (Schubert, 1999). This makes optimisation of the cell hydrodynamics for particle-bubble contacting virtually impossible, since the concentration of energy around the impeller usually results in high collision and detachment rates near the impeller and low collision rates in the bulk of the fluid (Koh \& Schwarz, 2003).

Despite these weaknesses, the robustness of the design has meant that mechanical cells overwhelmingly dominate in industrial applications, despite competition from several other cell technologies. Numerous studies have however shown that these cells may have considerable weaknesses in the flotation of fine particles since the flotation rate decreases significantly with decreasing particle size (Ahmed \& Jameson, 1985, Deglon, 
1998, Feng \& Aldrich, 1999, Pyke, 2004). This weakness is particularly acute in cases where slurries contain minerals which exhibit complex rheological behaviour (Deglon et. al., 2007, Nel et. al., 2007). High slurry viscosities cause a damping of the fluid turbulence which, according to flotation theory, decreases the collision rates in the cell (Schubert, 1999). Furthermore, the high slurry yield stress often encountered in fine ore slurries has been shown to result in "cavern formation" around the impeller (Moore \& Cossor, 1995, Fangary et. al., 2000, Arratia et. al., 2006) which can have an extremely negative effect on gas dispersion (Deglon et al., 2007). It is speculated that these problems are likely to become more acute in future, with mining companies seeking to recover ever finer and more complex ore types, thus providing greater scope for the application of novel cell technologies.

\subsubsection{Column Flotation Cells}

One of the first alternative cell designs to be developed was the column flotation cell which was first successfully trialled in the late 1960's (Boutin \& Wheeler, 1967). This design was based on the chemical engineering concept of a counter-current mass transfer system and was the first attempt to decouple the sub-processes of solid suspension, gas dispersion and particle-bubble contacting. The cell consists of a large column, usually 12 to $15 \mathrm{~m}$ high and approximately 1 to $5 \mathrm{~m}$ in diameter, where slurry is introduced near the top of the column and bubbles at the bottom of the column. The result is a quiescent, counter-current contacting system, as shown in the Figure 2.2: 


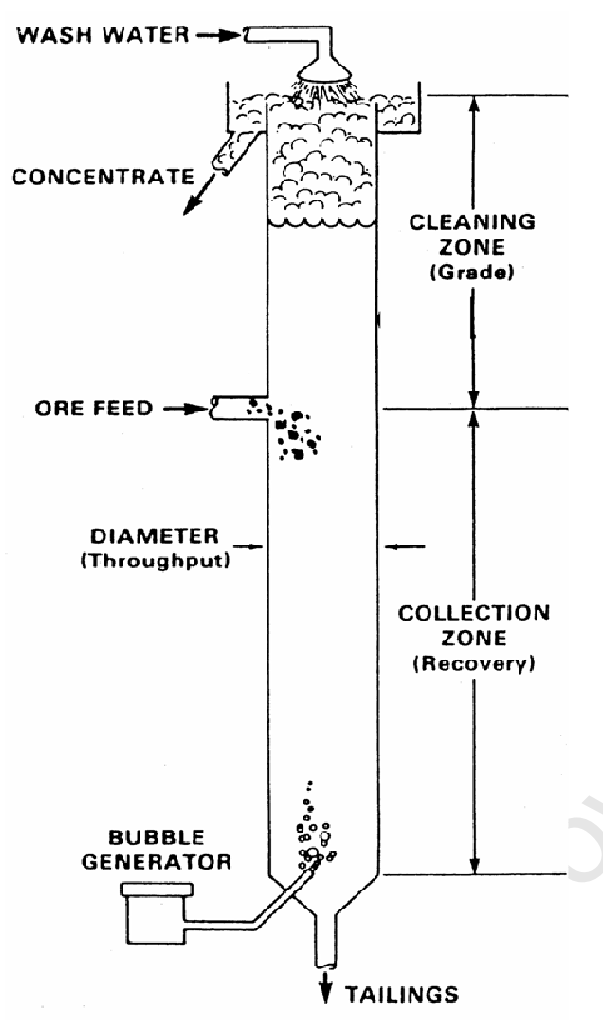

Figure 2.2 - Schematic of a Column Flotation Cell (McKay et. al., 1988)

The advantages of these devices include low installation and operating costs as well as the ability to operate at high froth depths, thus allowing significantly higher grade concentrates to be produced compared to a conventional cell.

The technology was slow to gain favour industrially and it was not until 1981 that the first commercial flotation column was installed at Les Gaspe (Quebec, Canada) for Molybdenum cleaning. It was found that one stage of column flotation was able to replace seven stages of conventional cell cleaning (Finch \& Dobby, 1990). Since then, the technology has gained popularity, particularly in base metal and coal applications.

Although column cells have achieved many successes industrially, they still suffer from two inherent flaws. Firstly, coarse particles have large settling velocities and therefore tend to have a shorter residence time in the cell, making them more difficult to recover. Secondly, fine particles generally lack the inertia to overcome the streamlines around 
bubbles and are therefore less likely to collide with approaching bubbles. Based on the particle Stokes number, it can be shown that particles in a quiescent system will follow fluid streamlines if (Jameson et. al., 1977):

$$
\frac{\rho_{p} d_{p}^{2} U_{b}}{9 \mu d_{b}} \square 1
$$

If one applies this equation to quartz particles being floated in a dilute suspension of water with a bubble size of $0.6 \mathrm{~mm}$ and assuming a bubble rise velocity of $7 \mathrm{~cm} / \mathrm{s}$ (Nguyen \& Schulze, 2004), the above inequality reduces to:

$$
d_{p} \square 175 \mu m
$$

For an order of magnitude estimate, one may assume that particles with a diameter less than 20 microns tend to follow the streamlines around bubbles and are therefore less likely to collide with approaching bubbles. Thus the fine particle fraction in most column cells is thought to have a reduced probability of collision.

For this reason, numerous modifications to column design have been proposed in order to improve fine and coarse particle recovery. One such technology is the static in-line mixer, which is used in the MicroCell ${ }^{\mathrm{TM}}$ flotation column (Yoon \& Luttrell, 1989, Yoon et. al., 1998). This apparatus improves contacting rates by reducing the bubble size in the column. The device works by pumping slurry out of the base of the column and contacting it with air in a high shear static mixer where a fine bubble-slurry mixture is formed. This mixture is then pumped back into the column where the bubbles rise quiescently through the system. A schematic of the apparatus is illustrated in the Figure 2.3: 


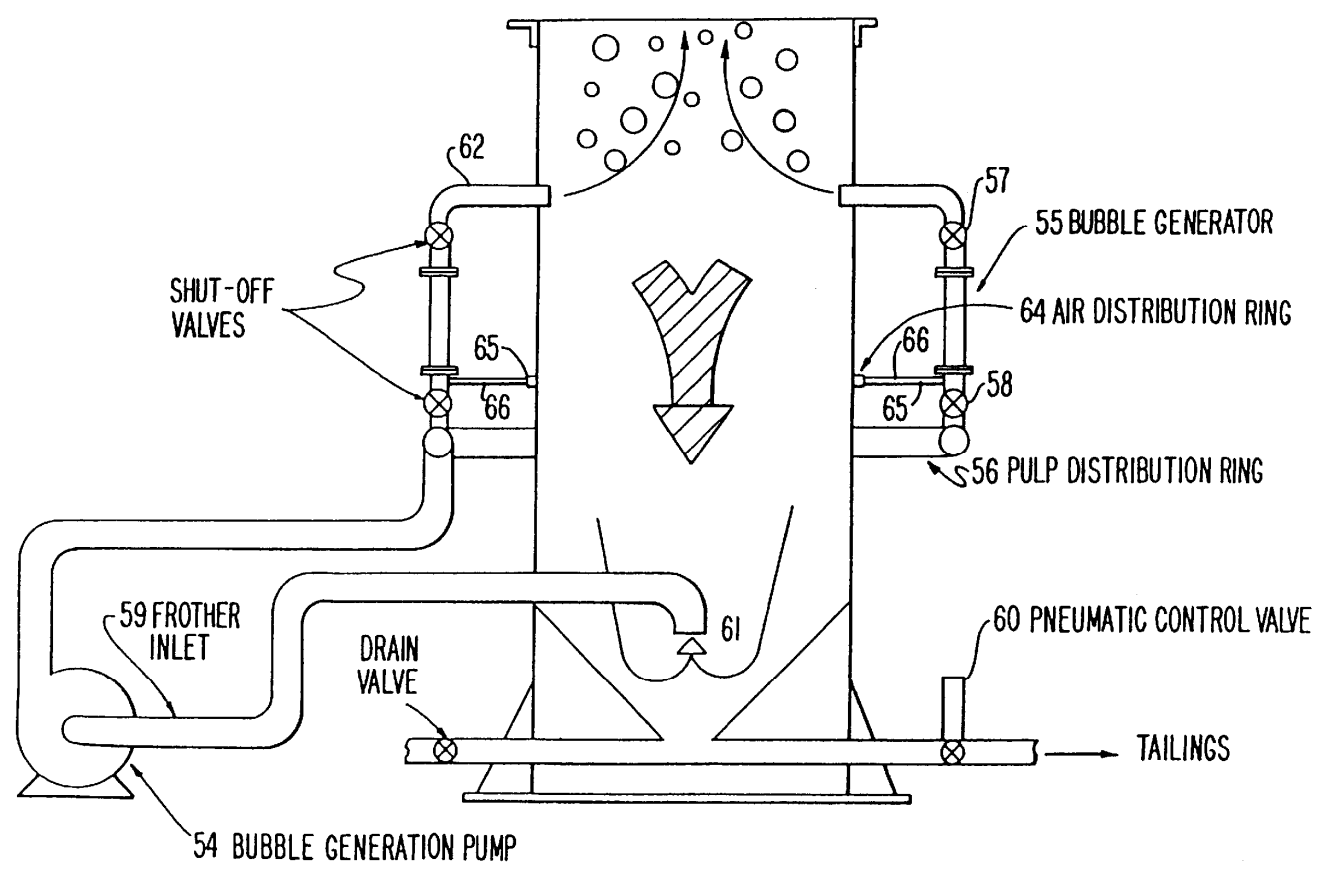

Figure 2.3 - Schematic of the Static In-line Mixer Configuration Used in the MicroCell ${ }^{\mathrm{TM}}$. (from Yoon et al., 1998)

Other improvements to column design include the addition of baffles to improve mixing and promote more intimate particle-bubble contact. Baffling has been placed in a number of configurations over the years, from vertical (Finch \& Dobby, 1990), to horizontal (Kawatra \& Eisele, 1993), to more sophisticated arrangements ( $\mathrm{Gu} \&$ Chiang, 1999). One notable extension of this principle is the development of the Packed Flotation Column (Yang, 1988). This column makes use of a series of tightly packed corrugated sheets which provide a tortuous path for both rising bubbles and descending particles, thus improving mixing and providing more intimate particle-bubble contact. The packing also enables a virtually unlimited froth depth which allows it to achieve exceptionally high upgrade ratios (Mohanty \& Honaker, 1999). A schematic of the packing configuration is shown in the Figure 2.4: 


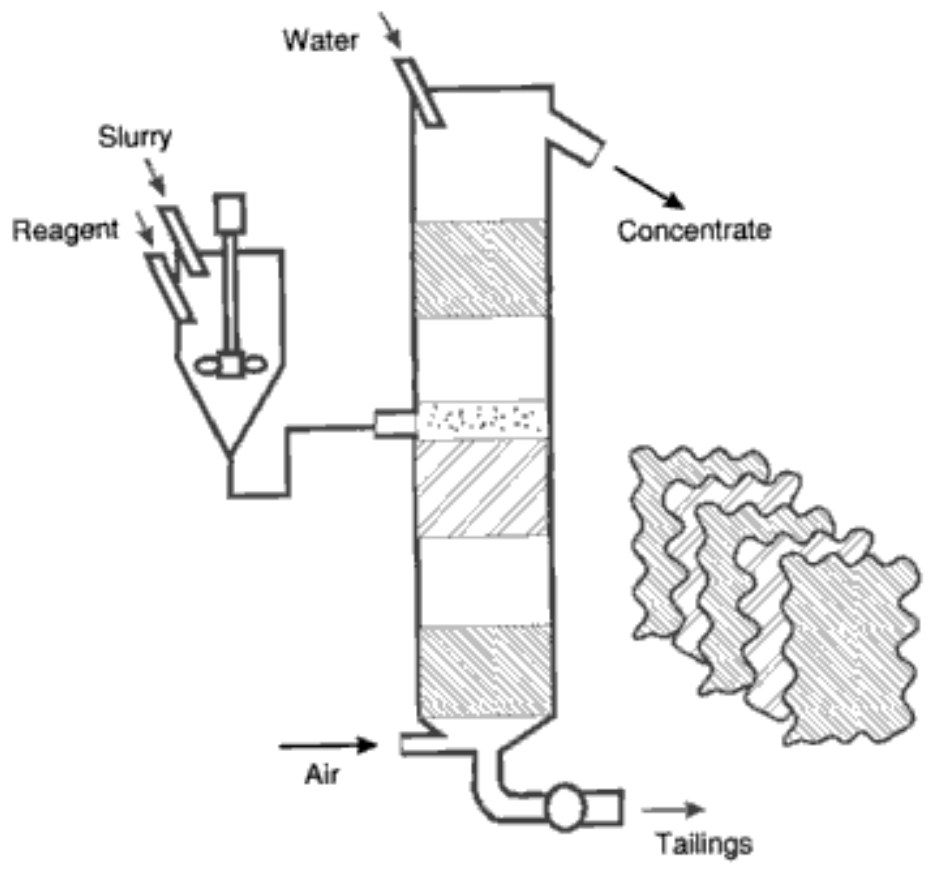

Figure 2.4 - Schematic of a Packed Flotation Column (from Rubinstein, 1995)

Although these improvements have achieved some successes, fine and coarse particle recovery remain an issue in most flotation columns today. For this reason most columns tend to find application as cleaners, rather than roughers, where they can be operated in a recycle configuration with mechanical cells to prevent the loss of valuable material.

\subsubsection{Novel Flotation Cells}

It is these problems with both fine and coarse particle recovery that have been the focus of many subsequent novel cell developments. Most of these systems attempt to improve the fine and coarse particle recovery by increasing the shear rate in the contacting zone, or by further decoupling the collection and cleaning functions of the cell. The following section presents a review of some of the major types of novel cells and highlights the designs which have found particular industrial success. 


\subsubsection{Agitated Columns}

One of the first attempts to improve fine and coarse particle recovery in column flotation was the introduction of agitated flotation columns. One of the first examples of this was the HydroChem column (Schneider \& Van Weert, 1988), which consisted of a standard flotation column fitted with a series of alternating impellers and spinning discs down its length. The configuration was designed to create a tanks-in-series type mixing profile down the column length. This column achieved some success but failed to improve coarse particle recovery due to the high detachment rates associated with spinning discs. The researchers noted an optimum impeller rotation speed of $250 \mathrm{rpm}$ when treating a chalcopyrite ore.

Later, Harris et. al. (1992) tested a hybrid flotation cell, which consisted of a Leeds batch cell design combined with a 2 inch column section fitted to the top of the cell. This design was found to significantly improve coarse particle recovery relative to a standard column cell and was able to produce a much higher grade than a batch flotation cell.

This work was then extended by Breytenbach (1995), who evaluated the performance of a mechanically agitated column cell on quartz flotation. This cell consisted of a standard flotation column fitted with 4 impellers down its length. It was evaluated relative to the performance of other cell technologies, viz. a standard column cell, a Jameson cell and a batch flotation cell. Some key results of this study are shown in Figure 2.5: 


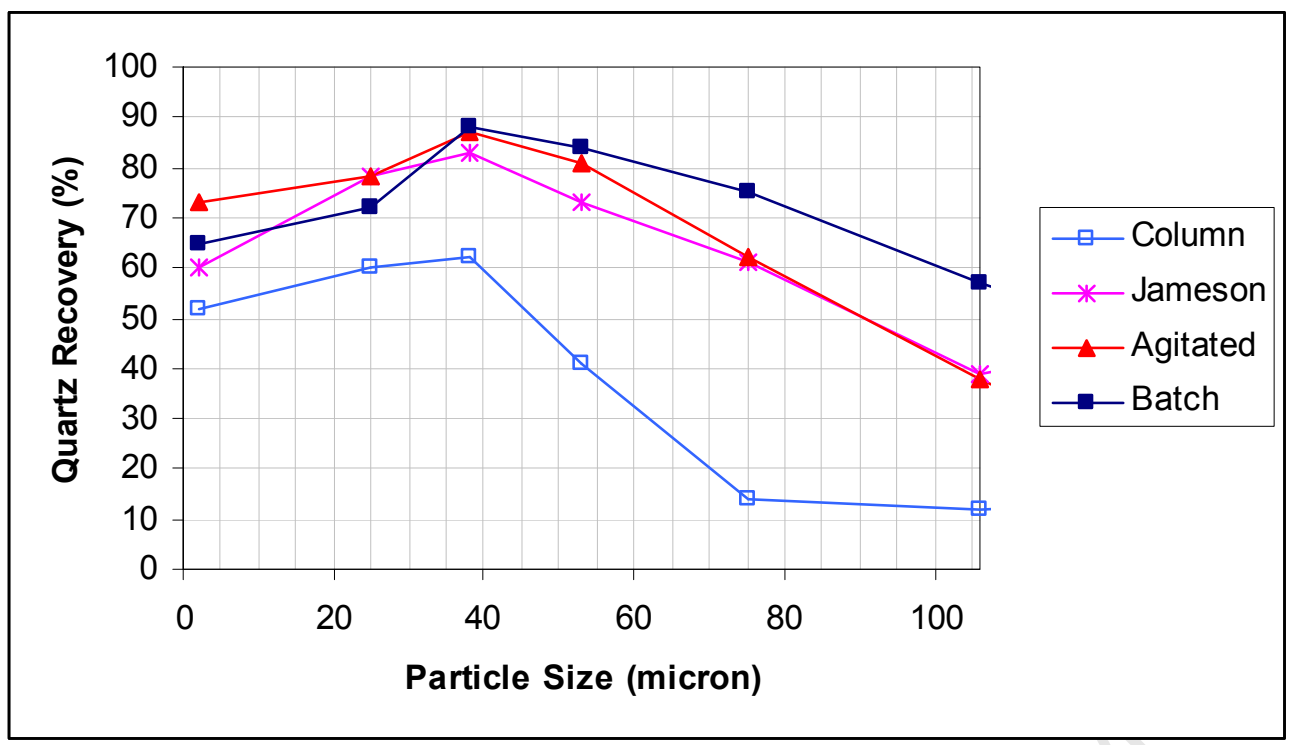

Figure 2.5 - Flotation Performance of an Agitated Column Cell relative to other Flotation Cell Technologies as a Function of Particle Size (from Breytenbach, 1995).

Figure 2.5 shows that the agitated column was able to produce good flotation recoveries relative to the other flotation cell types, particularly for fine particles. This improved recovery was attributed to enhanced particle-bubble contacting under agitated conditions. Figure 2.5 also illustrates the comparatively low recoveries obtained in a standard column for fine and coarse particles due to the weaknesses discussed in section 2.1.2.

Based on these findings, Ityokumbul et. al. (2000) investigated the performance of the same agitated column in the flotation of a fine gold-bearing pyrite ore, with a particle size distribution of $62 \%$ passing 25 microns. A comparison between the flotation results of Breytenbach (1995) and Ityokumbul et. al. (2000), as a function of power input to the system, is shown in Figure 2.6*:

\footnotetext{
${ }^{*}$ Specific power inputs are calculated based on the electrical power draw of the motor at a given agitation speed (from Ityokumbul et. al., 2000).
} 


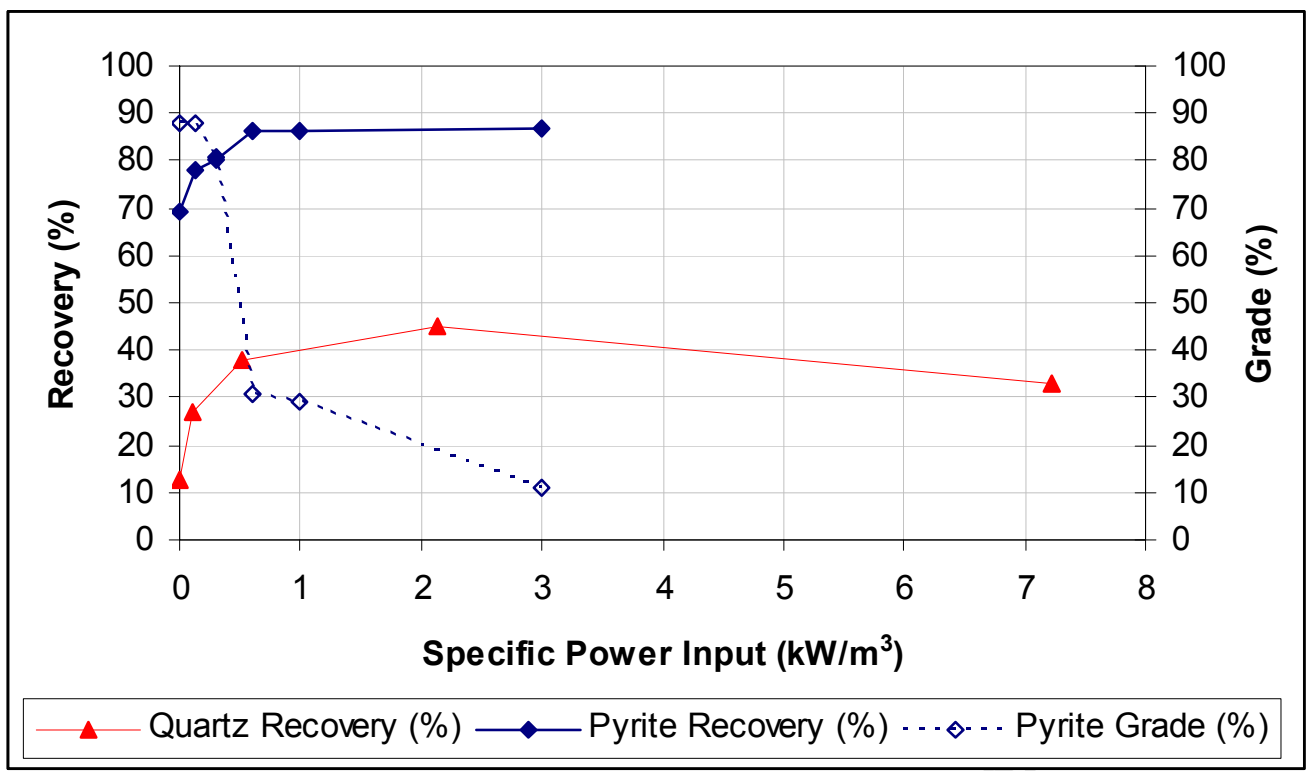

Figure 2.6 - Flotation Performance of the Agitated Column as a Function of Specific Power Input for Flotation of +106-150 micron Quartz (from Breytenbach, 1995) and Flotation of Fine Pyrite (62\% passing 25 micron) (from Ityokumbul et al., 2000).

Figure 2.6 shows that the addition of energy to the system produced a substantial increase in the flotation recovery relative to a standard column for both quartz and fine pyrite flotation. As power input was increased beyond approximately $1 \mathrm{~kW} / \mathrm{m}^{3}$, the recovery started to level off and eventually decreased as the power input was increased to $7.2 \mathrm{~kW} / \mathrm{m}^{3}$. Interestingly, the concentrate grade was found to decrease sharply at a power input of $0.31 \mathrm{~kW} / \mathrm{m}^{3}$. This was most likely due to an increase in entrainment under these conditions and it was therefore concluded that a power input of $0.31 \mathrm{~kW} / \mathrm{m}^{3}$ was optimal.

The agitated column was therefore concluded to be highly successful in the flotation of fine pyrite particles and was able to produce a $13 \%$ increase in recovery relative to a column flotation cell at comparable concentrate grade.

Despite this evidence of significant improvements in flotation rate, agitated columns have yet to be tested industrially. Some exceptions were the earlier column designs such as the WEMCO/Leeds cell (Degner \& Sabey, 1988) and the OK cell (Ulan et. al., 1991) 
of the late 1980's and early 1990's which incorporated agitation into their designs. These cells were applied in limited instances but were distinctly different in their design compared with the aforementioned agitated columns, in that the impeller was located at the base of the column as in a conventional cell. The role of the impeller was more for promoting better gas dispersion and solid suspension than for optimising particle-bubble contacting. For this reason the cells only achieved limited success and were eventually replaced by conventional cell technologies.

Given the significant improvements in fine particle recovery achievable using agitated columns, it is believed that a distinct opportunity still exists for the application of this technology to industrial slurries. It is speculated that such a column, combined with a modern micro-bubble generation apparatus, such as a static mixer, may be able to achieve exceptional fine particle performance by optimising the energy input independently of gas dispersion effects.

\subsubsection{Reactor/Separator Type Cells}

Another major development in flotation cell design was the development of "reactorseparator" type cells. These cells work by separating the contacting and cleaning functions of the cell, thus allowing independent optimisation of each function. The literature is filled with many examples of such designs such as the Flotaire Cell (Gruber \& Kelahan, 1988), the Pneumatic Cell (Changgen \& Bahr, 1992), the Contact cell (Amelunxen, 1993) and the LM flotation cell (Xinghau, 1998), but perhaps the bestknown and most successful design is the Jameson cell (Jameson, 1988), shown in Figure 2.7: 


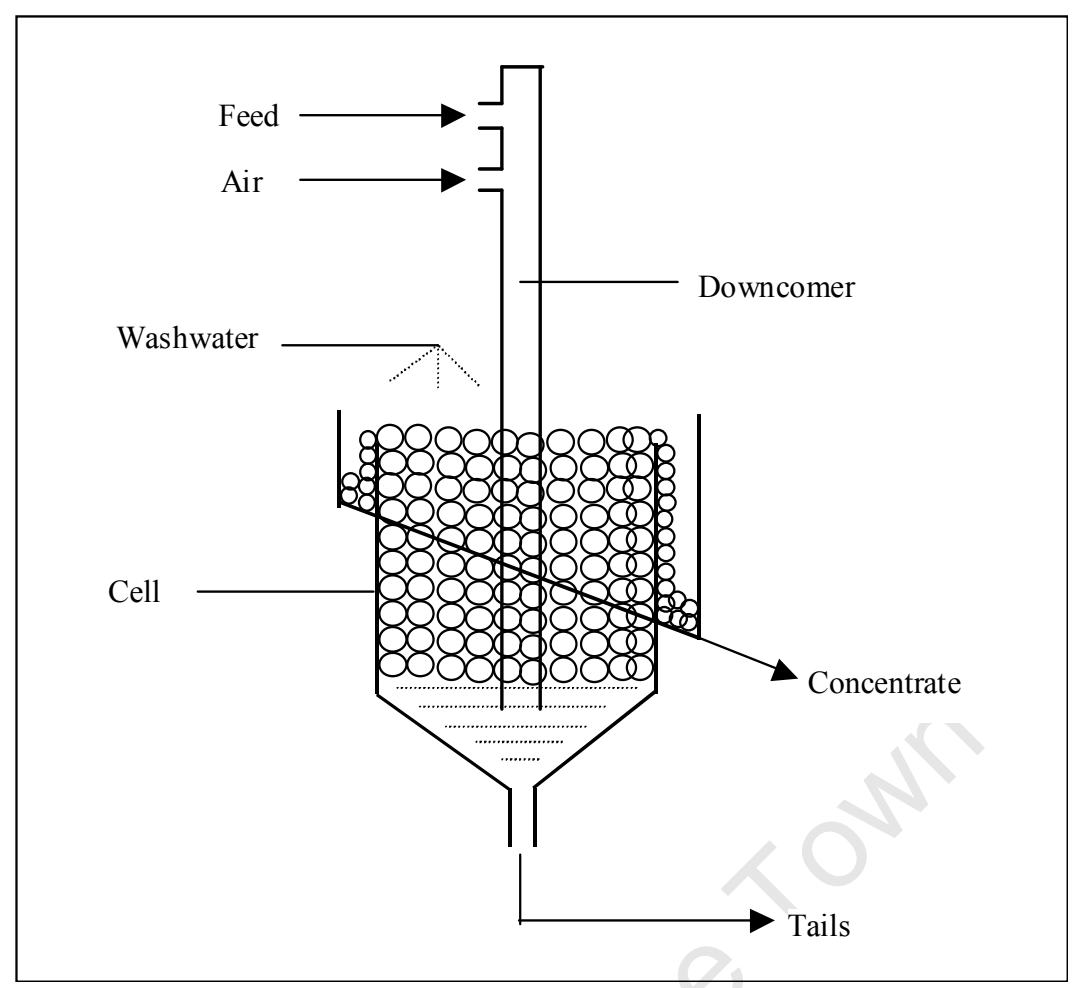

Figure 2.7 - Schematic of a Jameson Flotation Cell (from Rubinstein, 1995).

The cell consists of one or more vertical downcomer tubes through which slurry and entrained air is introduced at high velocity. This results in a highly turbulent, high gas hold-up contacting zone in which bubbles and particles are intensely mixed. The slurry is then rapidly ejected into a quiescent disengagement zone where a deep froth is formed to yield a high grade concentrate.

Since the first installation at Mount Isa Mines in 1986, the Jameson cell has achieved many successes and there are currently more than 228 installations worldwide (Cowburn et. al., 2005). The design has also been extensively developed since its first introduction and modern Jameson cells consist of up to 20 parallel downcomers incorporating sophisticated slurry injection systems which maximise air entrainment (Jameson, 2007).

Jameson cells have been shown to be particularly effective in fine coal flotation, and are able to produce high throughputs when compared with MicroCell $^{\mathrm{TM}}$ and Packed 
Column technologies (Mohanty \& Honaker, 1999). Their weakness lies in the short contacting time of the slurry in the downcomer, which is usually of the order of 10 seconds. This means that weakly hydrophobic particles only have a limited opportunity to successfully contact bubbles and be recovered. The cell design is therefore thought to be best suited to the flotation of highly hydrophobic material such as coal and chalcopyrite. In addition the cells require a highly stable feed rate and good control systems to prevent fluctuations in performance. This weakness has however been somewhat mitigated in recent designs by incorporating a slurry recycle to stabilise the feed rate.

\subsubsection{Other Novel Devices}

Many other novel flotation devices have been suggested over the years - mostly in the waste water treatment and paper deinking industries where flotation is used for the removal of waste products. Comprehensive reviews of such technology are given in Finch \& Hardie (1999) and Rubio et. al. (2002). Notable examples include the cells such as the Centrifloat ${ }^{\circledR}$ and the Air-Sparged Hydrocyclone (Ye et. al., 1988), which make use of the shear rate under a centrifugal flow field to induce greater particlebubble contacting. Other novel techniques include electro flotation and dissolved air flotation for the generation of microbubbles, and even cavitation air flotation which attempts to seed bubbles directly onto particle surfaces.

\subsubsection{SWOT Analysis of Flotation Cell Types}

In order to assess the efficacy of the various flotation cell designs reviewed in this chapter, the following section analyses each cell's effectiveness in fine particle recovery using the SWOT Analysis technique. The SWOT analysis is not exhaustive but serves to contextualise agitated columns relative to other cell technologies. The analysis is summarised in Table 2.1: 


\begin{tabular}{|c|c|c|c|c|}
\hline 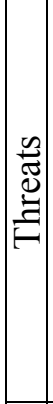 & 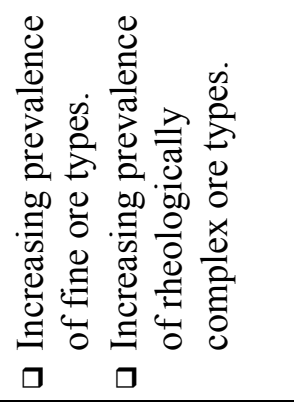 & 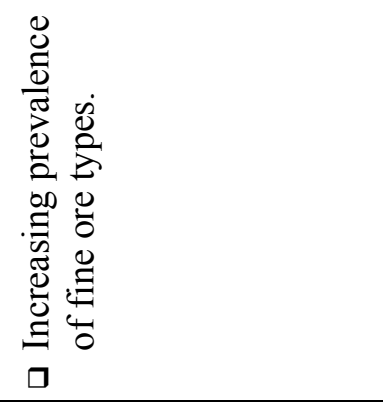 & 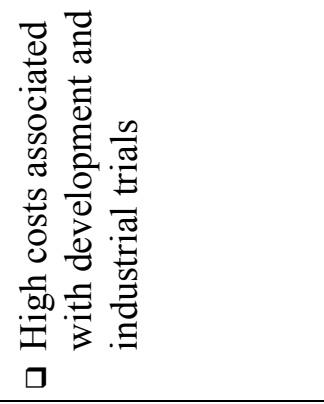 & 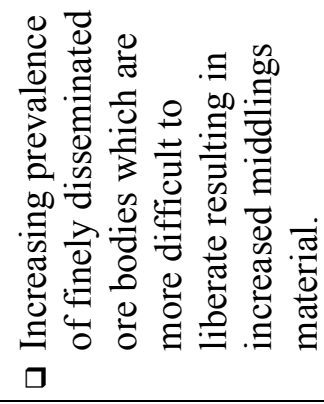 \\
\hline 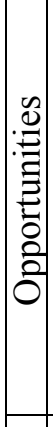 & 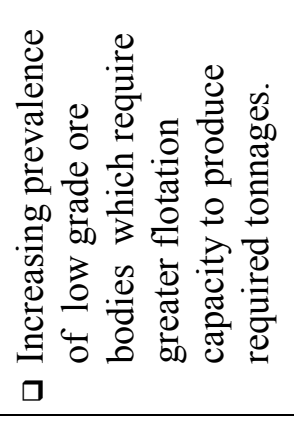 & 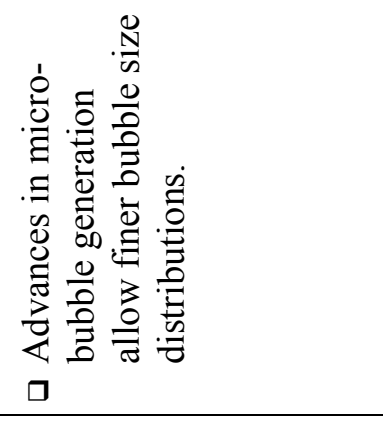 & 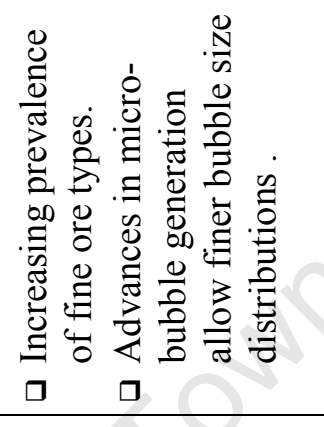 & 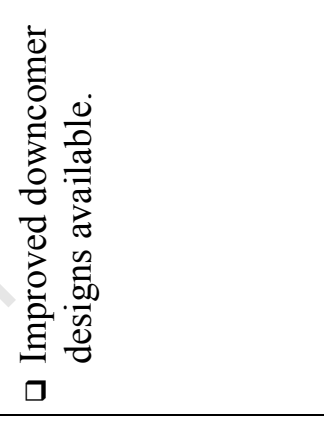 \\
\hline & 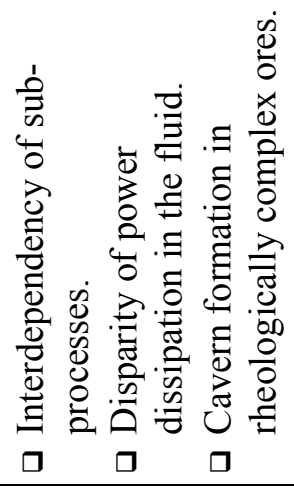 & 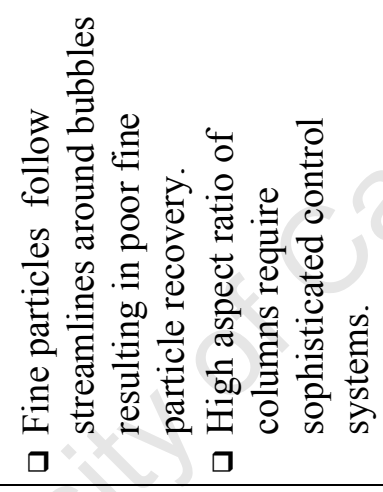 & 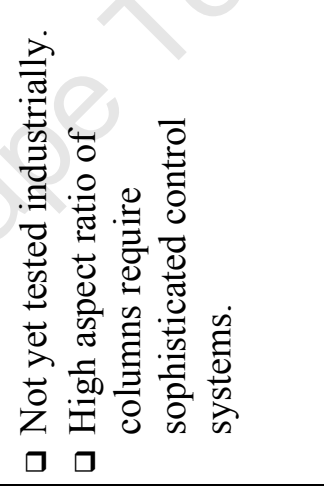 & 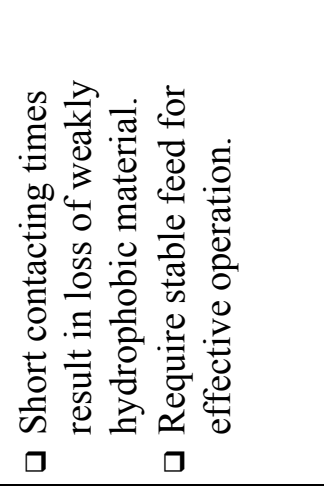 \\
\hline 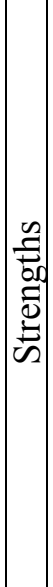 & 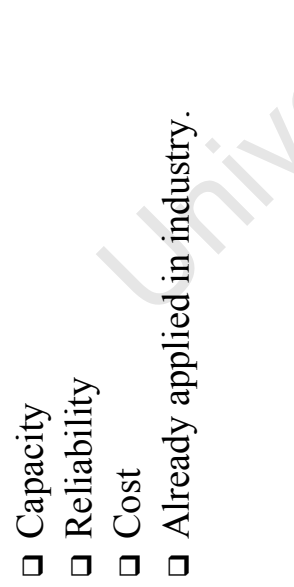 & 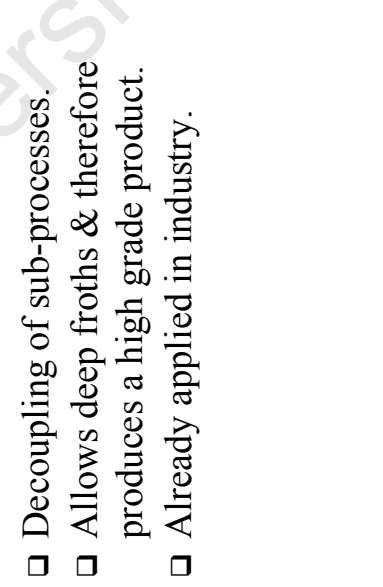 & 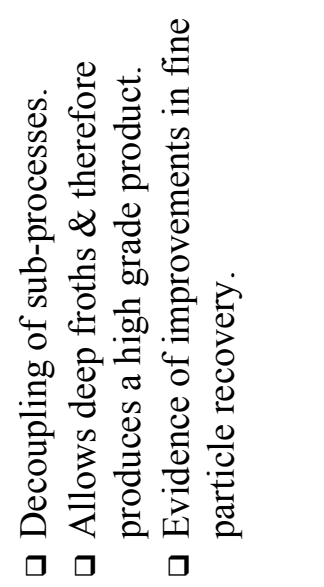 & 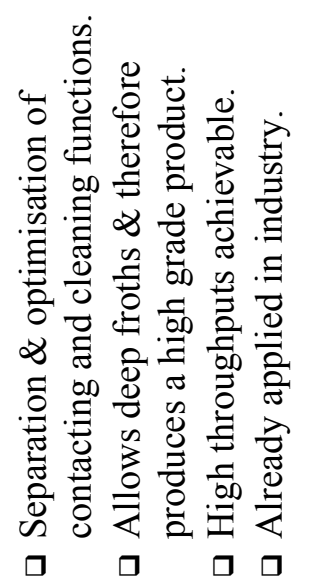 \\
\hline & 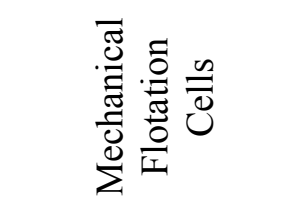 & 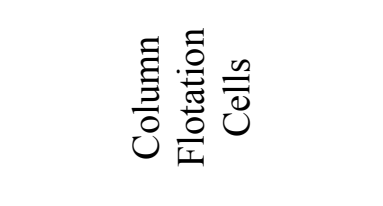 & 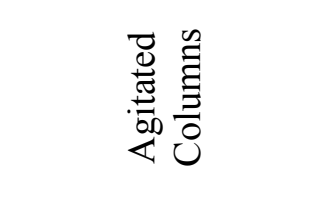 & 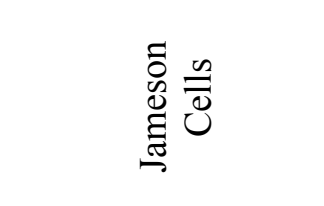 \\
\hline
\end{tabular}


The analysis in Table 2.1 illustrates that the two most prevalent flotation cell designs, the mechanical cell and the column flotation cell, are under threat from the increasing prevalence of fine mineral slurries in modern-day flotation applications. While reactorseparator cells can be effective in the flotation of fine mineral slurries, they are limited by the short contacting time available for particle-bubble contacting and are therefore best suited to the treatment of highly hydrophobic material which readily attaches to bubble surfaces. However as mining companies are forced to turn to increasingly low grade, fine disseminated ore types, the liberation of valuable minerals becomes more difficult, leading to an increased prevalence of weakly hydrophobic middlings particles.

The work of Breytenbach (1995) and Ityokumbul et. al. (2000) has provided evidence that the agitated column design is effective in the treatment of fine mineral slurries and may be able to cope with the fine material which is becoming increasingly prevalent in the minerals processing industry. Despite this evidence, these cell types have yet to be trialled industrially and the costs associated with their development may be prohibitive in some applications.

This thesis aims to build on the strengths of the agitated column design, by introducing a new cell design to the flotation literature. This cell also incorporates the agitated column's ability to decouple the sub-processes of bubble generation and particle-bubble contacting. However, it employs a different mode of agitation which has been highly successful in other industrial applications, particularly where performance is dependent on the shear rate in the fluid. These cells are known as Oscillatory Baffled Columns (OBC's) and have been applied extensively in industries such as bio-processing, pharmaceuticals and solvent extraction. The following section reviews the key features of Oscillatory Baffled Columns (OBC's) in terms of their mixing behaviour, fluid dynamics and power characteristics, before assessing their potential strengths and weaknesses in application as novel flotation cells. 


\subsection{Oscillatory Baffled Columns}

\subsubsection{Background}

Oscillatory Baffled Columns are a relatively new type of mixing device which have been studied in many multi-phase mixing applications. The OBC design consists of a standard cylindrical column section with a series of evenly spaced orifice plates mounted down its length. A sinusoidal motion is then superimposed on the fluid, either by pulsing the fluid, using a piston or bellows, or by oscillating the plates themselves through the fluid, using a piston and cam. This design allows considerable variability of the power input to the cell, since one is able to vary not only the frequency of the power input but the amplitude as well.

The origins of this device can be traced back to the reciprocating plate columns developed in the nuclear industry for liquid-liquid extraction (Van Dijck, 1935). These columns were widely studied in the 1950's and many design variations have been noted in the literature (Sege \& Woodfield, 1954, Karr, 1959). The most common design adjustment was the baffle plate design which usually consisted of sieve plates with multiple holes in their cross section.

These plates remained popular for many years until studies in the early 1980's started to show that oscillatory flow through sharp edged ducts were able to produce strong vortex rings which could provide effective radial mixing (Knott \& Mackley, 1980). This led to the replacment of the traditional sieve plates with a series of evenly spaced orifice baffles (Brunold et. al., 1987), leading to the development of OBC's as they are known today.

The late 1980's and 1990's saw a flourish of research to characterise OBC's and illustrate their strengths and weaknesses. Many papers published data on the flow patterns and mixing characteristics that could be achieved in the system (Brunold et. al., 1989, Dickens et. al., 1989, Mackley \& Ni, 1991, Mackley \& Ni, 1993). This literature showed that OBC's could exhibit highly plug flow conditions at low frequencies and amplitudes and could also provide good global mixing characteristics at high 
frequencies and amplitudes. This improved plug flow performance allowed significant enhancements to heat transfer (Mackley et. al., 1990, Mackley \& Stonestreet, 1995), mass transfer (Jaeger, 1997, Ni et. al., 1997, Thomas \& Narayanan, 2002) as well as chemical reaction applications (Ni \& Mackley, 1993, Stonestreet \& Harvey, 2002, Skelton et. al., 2005).

The significant control and variability of the hydrodynamic environment in the $\mathrm{OBC}$ has allowed the design to be successfully applied to numerous industrial processes, particularly where performance is highly dependent on the shear rate in the fluid. These include bio-fermentation (Ni et. al., 1995, Gaidhani et. al., 2003), flocculation (Gao et. al., 1998), suspension polymerisation (Ni et. al., 1999), protein refolding (Lee et. al., 2002), photocatalytic oxidation (Fabiyi \& Skelton, 1999, Gao et. al., 2003) and droplet breakage (Mignard et. al., 2003).

OBC's are currently gaining much popularity in bio-processing applications due their ability to provide good mixing under relatively low shear conditions. They are also finding extensive application in the pharmaceuticals industry where their excellent plug flow characteristics are being exploited to allow continuous production of pharmaceutical products at extremely small scales.

\subsubsection{Mechanism of Mixing in an OBC}

The mechanism of mixing in an $\mathrm{OBC}$ can be understood with the help of the following diagram: 


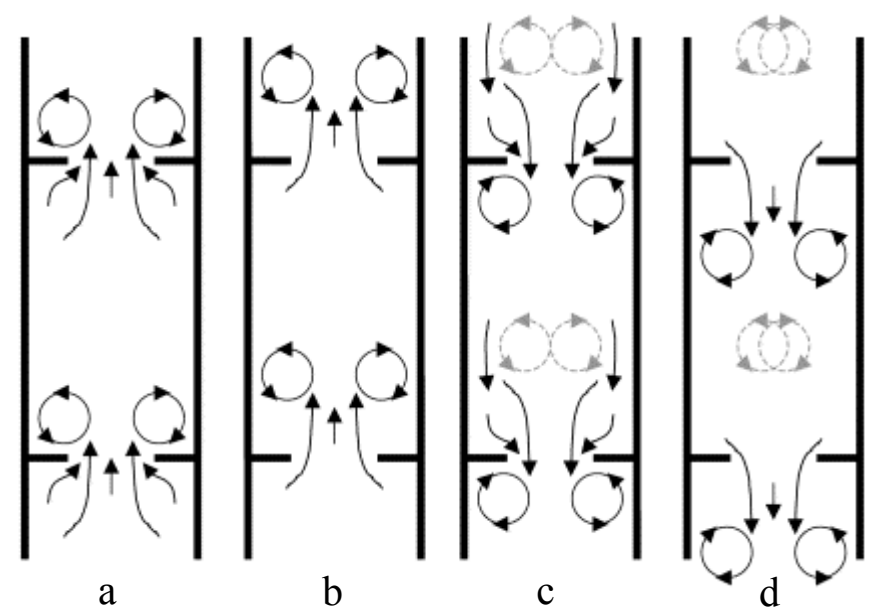

$$
\begin{aligned}
& \mathrm{a}=\text { Start of up stroke } \\
& \mathrm{b}=\text { Maximum velocity in up stroke } \\
& \mathrm{c}=\text { Start of down stroke } \\
& \mathrm{d}=\text { Maximum velocity in down stroke }
\end{aligned}
$$

Figure 2.8 - Mixing Mechanism in an OBC (from Ni \& Jian, 2005)

When fluid is accelerated through the baffle constriction, tore-shaped vortices are formed behind the baffles. As the fluid starts to decelerate, these vortices shed and are swept into the centre of the baffle cavity by the jet of incoming fluid. The vortex then begins to weaken and acts as an obstacle to the incoming jet of fluid during the down stroke (c.f. Figure 2.8).

Recent studies by Fitch (2003) have indicated that the amplitude of oscillation controls both the size of the vortices formed and the distance which they are propagated into the inter-baffle cavities. The frequency, in contrast, affects the maximum velocity obtained within the vortices but does not affect their size or propagation.

Over the years, these complex mixing patterns have been characterised by researchers as a function of a number of dimensionless numbers. The following section details the structure and formulation of these numbers. 


\subsubsection{Characteristic Dimensions and Dimensionless Groups}

Figure 2.9 illustrates the key parameters used to characterise flow in an OBC:

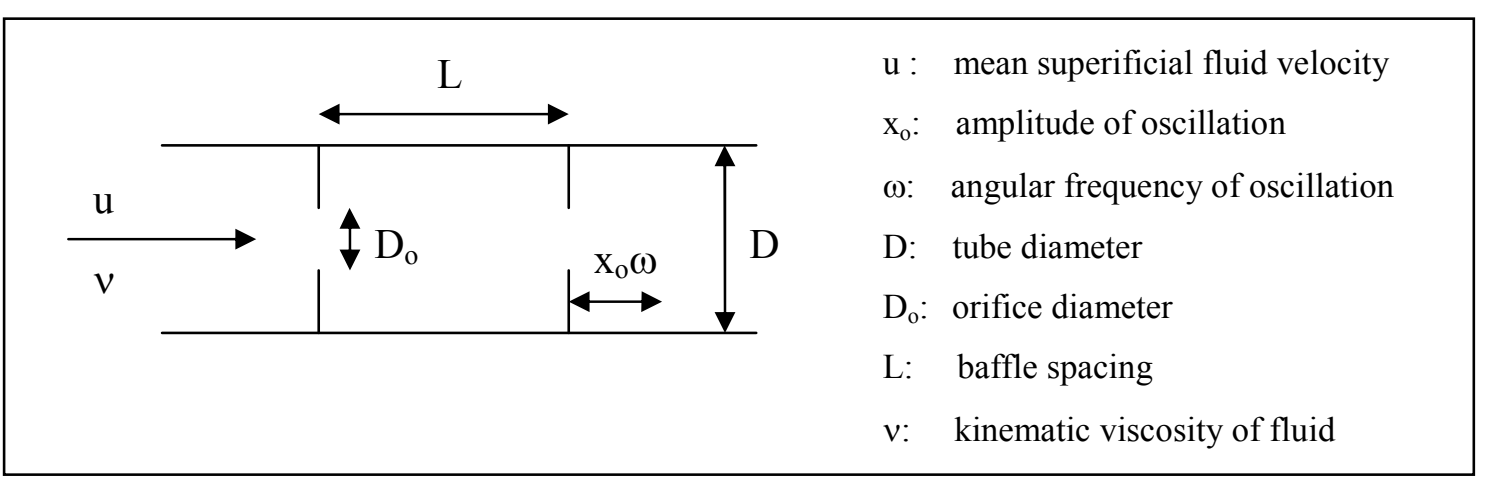

Figure 2.9 - Characteristic Dimensions of an Oscillatory Flow System

As can be seen from the above figure, there are a total of 7 variables in oscillatory baffled flow (OBF). These include 3 operating parameters $\left(\mathrm{u}, \mathrm{x}_{0}, \omega\right), 3$ geometric parameters $\left(D, D_{0}, L\right)$ and 1 fluid parameter $(v)$. These dimensions can be grouped into 5 dimensionless numbers which characterise oscillatory flow:

Table 2.2: Dimensionless Groups Describing Flow in an OBC (Ni \& Gough, 1997)

\begin{tabular}{|l|l|}
\hline Net flow Reynolds' number: & $\operatorname{Re}_{n}=\frac{D \cdot u}{v}$ \\
\hline Oscillatory Reynolds' number: & $\operatorname{Re}_{o}=\frac{D \cdot x_{0} \cdot \omega}{v}$ \\
\hline Strouhal Number: & $S t=\frac{D}{4 \pi \cdot x_{o}}$ \\
\hline Baffle Spacing: & $(L / D)$ \\
\hline Baffle Free Area: & $S=\left(\frac{D_{o}}{D}\right)^{2}$ \\
\hline
\end{tabular}


Here, the oscillatory Reynolds number is a measure of the intensity of mixing, and the Strouhal number is the inverse of amplitude and is an indication of the effective eddy propagation through the system (Ni \& Gough, 1997). Thus the smaller values of St, indicate greater propagation of eddies into the inter-baffle cavities.

The baffle spacing and baffle free area are design parameters which are given as heuristics in the literature. Baffle spacings generally range between 1 and 2 column diameters and baffle free area, S, is usually in the range of 0.2 - 0.5 (Harvey et. al., 2001). Brunold et. al. (1989) showed that a baffle spacing of 1.5 times the tube diameter and a baffle free area of 0.4 gave optimal mixing conditions between adjacent baffles.

Harvey et. al. (2001) outlined some distinctive flow regimes which are thought to occur in the $\mathrm{OBC}$ as a function of oscillatory Reynolds number. In contrast to steady flows where flow separation occurs at nett flow Renolds number of 2100, flow separation in oscillatory flow occurs at an oscillatory Reynolds number between 50 and 100. For $\operatorname{Re}_{\mathrm{o}}$ in the range of $50-500$, a "soft" mixing pattern is generated in which the flow is axisymmetric and relatively ordered in nature. These conditions produce low shear rates in the fluid and good plug flow behaviour. As the oscillatory Reynolds number is increased, the flow starts to become more chaotic and becomes non-axisymmetric from $\mathrm{Re}_{\mathrm{o}} \sim 1600$ (Ni et. al., 2000). From $\mathrm{Re}_{\mathrm{o}}=5000$, the flow becomes intensely mixed and starts to approximate continuous stirred tank reactor (CSTR) behaviour.

The following section will examine the fluid dynamic behaviour of OBC's more closely, with specific emphasis on the shear rate distribution in the fluid and how this is affected by fluid parameters such as viscosity and yield stress. This is because the shear rate in the fluid is thought to be a particularly important driver of particle-bubble contacting in flotation. The results of studies examining the behaviour of particles under oscillatory flow are also reviewed. 


\subsubsection{Fluid Dynamics of an OBC}

Recent improvements in computational power have allowed considerable advances in the fields of Computational Fluid Dynamics (CFD) and Particle Image Velocimetry (PIV). These advances have in turn provided researchers with powerful tools to investigate the fluid dynamics of OBC's and map them in terms of their velocity and shear rate distributions. The shear rate distribution within a flotation cell is considered to be of particular importance in its design, since the shear rate is thought to affect the collision and detachment rates within the cell, as will be discussed in section 2.3.

Recently, Bakker (2005) developed a CFD model of an OBC using Large Eddy Simulation (LES). The experimental data of Ni et. al. (2000) was used to validate the model and good agreement was obtained between the observed and simulated flow patterns. Figure 2.10 shows a snapshot of the results of the simulation showing the spatial distribution of shear rates in the fluid. Note that only the central region between the adjacent baffles simulates the agitated zone of an OBC.
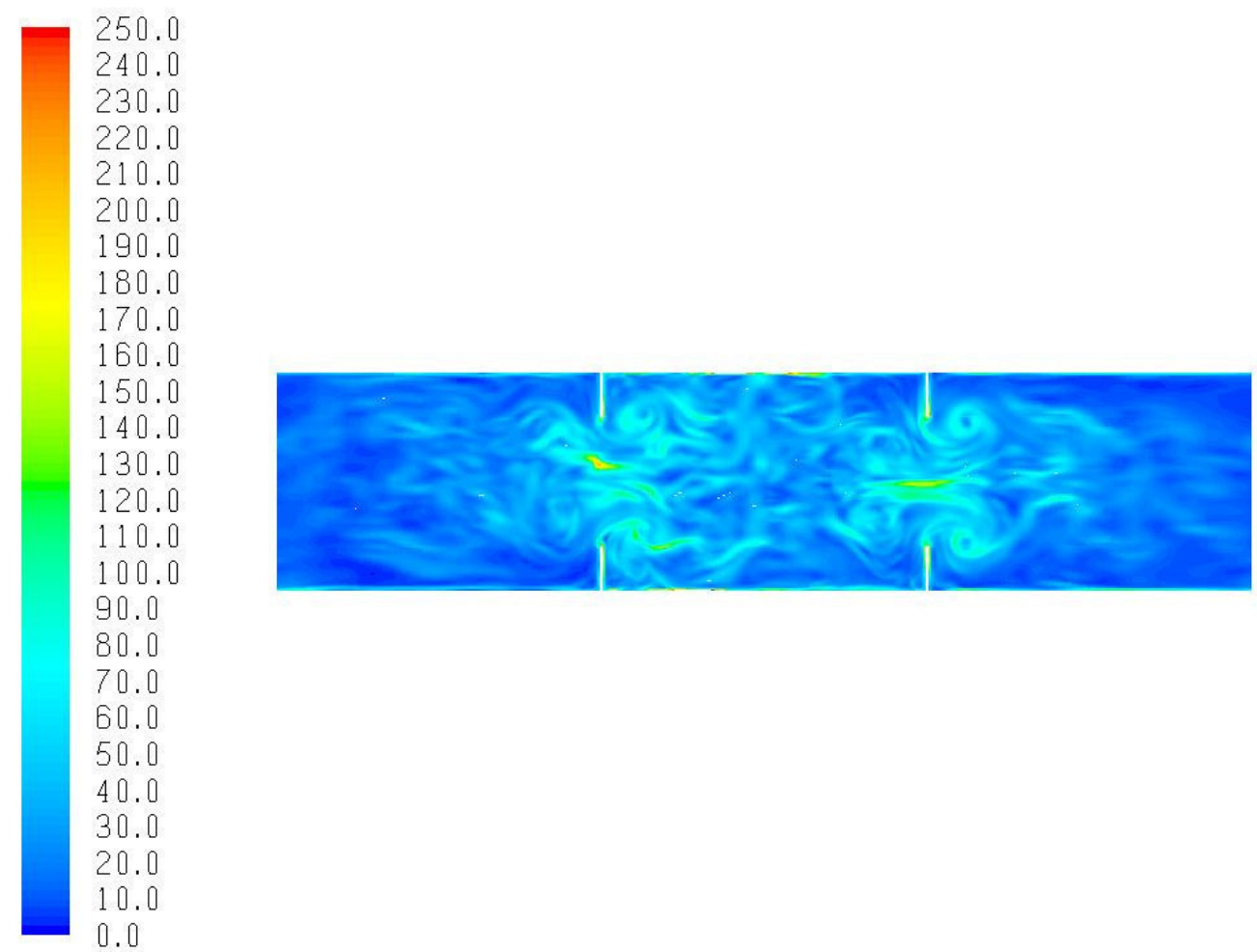

Figure 2.10 - Shear Rate Distribution within an OBC Oscillating at $5 \mathrm{~mm}$ and $2.5 \mathrm{~Hz}$, taken at the Point of Maximum Fluid Velocity $\left(\mathrm{Re}_{\mathrm{o}}=\mathbf{7 8 5 0}\right)$ (Bakker, 2005). 
As can be seen in Figure 2.10, the OBC provides a particularly even distribution of shear rate through the fluid, particularly in the inter-baffle cavities. While some spatial variation can be observed, with shear rates being concentrated in the "jet" and "vortex" structures of the flow, the overall variance of shear rate through the fluid is not large. These results are in good agreement with the results of $\mathrm{Ni}$ et. al. (2000), who found similar flow patterns under different oscillatory conditions. This is in stark comparison to the shear rate distribution obtained in conventional stirred system, such as the example shown in Figure 2.11.
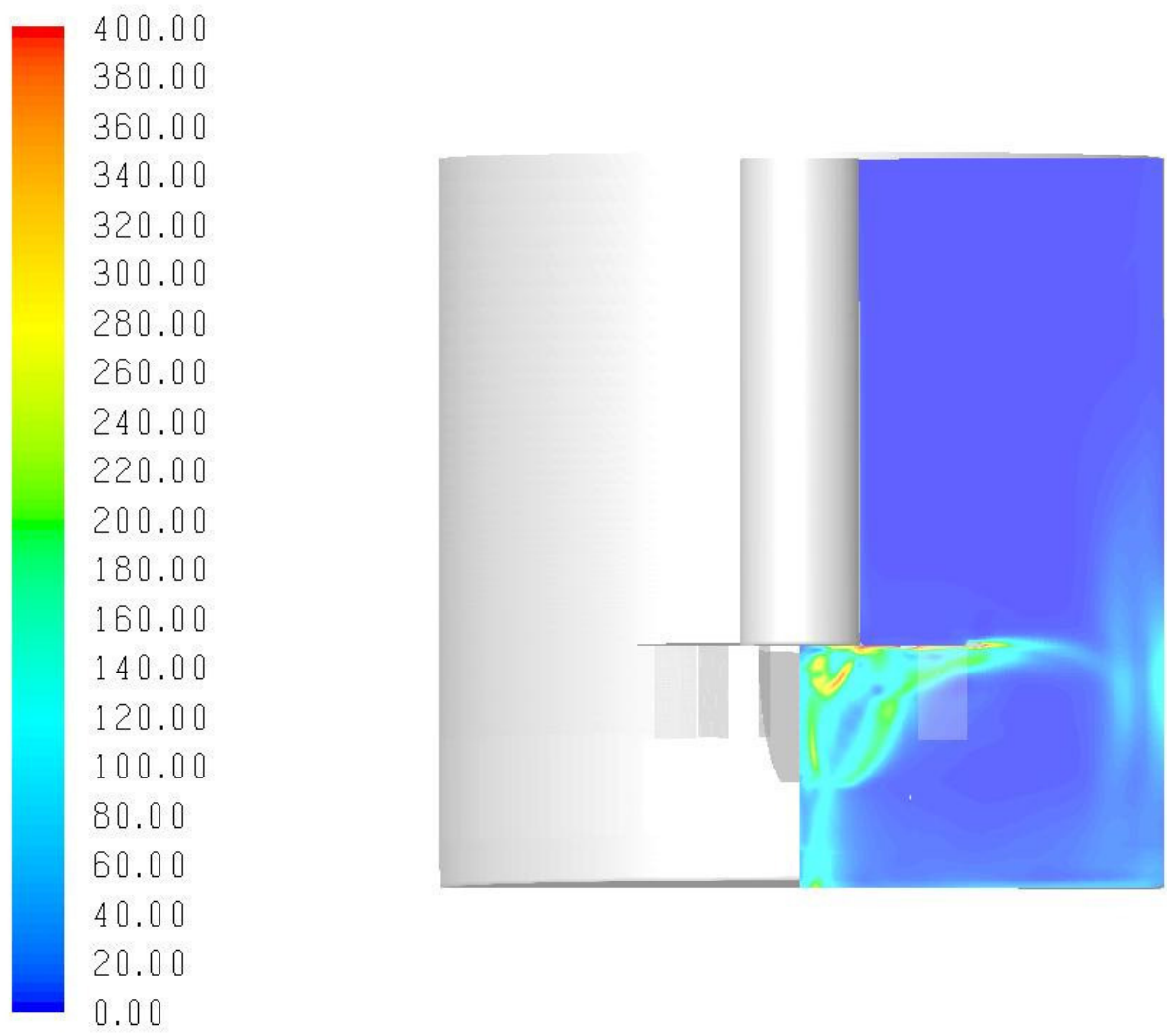

Figure 2.11 - Shear Rate Distribution in a Bateman 120 litre Pilot Flotation Cell operating at $400 \mathrm{rpm}\left(\operatorname{Re}=1.5 \times 10^{5}\right)($ Bakker, 2008).

Figure 2.11 shows the considerable disparity of the shear rate distribution within a conventional stirred system, where the shear rate is concentrated in a small region around the impeller. The rest of the fluid in contrast is relatively quiescent and under 
very low shear conditions. Comparison of Figures 2.10 and 2.11 clearly illustrates that the shear rate distribution with an $\mathrm{OBC}$ is considerably more evenly distributed compared with a conventional cell.

It should be noted that Figures 2.10 and 2.11 are not intended to be a direct quantitative comparison between the cells but rather aim to illustrate the difference in flow structures which apply in either system. These flow structures are largely determined by the geometry and agitation mechanism of the cell rather than the intensity of the agitation. Similar examples of these flow patterns have been reported by numerous researchers (Fitch \& Ni, 2003, Koh \& Schwarz, 2003, Tiitinen, 2003, Chew et. al., 2004). Although the magnitude of the shear rates observed certainly varies with varying agitation levels, the overall structure of the shear rate distribution in each system does not change dramatically. Figures 2.10 and 2.11 therefore illustrate that the shear rate produced under oscillatory flow conditions is relatively evenly distributed through the volume of the cell, rather than being concentrated in a relatively small volume around the impeller.

This is further supported by other studies in mechanical flotation cells have shown that the energy dissipation rate at the rotor ranges between 5 and 200 times the average power input to the cell (Schubert, 2007). This disparity leads to an enormous difficulty in optimising the particle-bubble contacting rate in the cell, since particles and bubbles are exposed to an enormously wide range of shear conditions with only a small volume of the cell exhibiting optimal shear conditions. In contrast the OBC provides a considerably more even distribution of shear rate in the fluid, meaning that particles are exposed to a much narrower range of shear rates. The OBC also provides considerable control over the magnitude of the shear rate distribution since one is able to vary both the amplitude and frequency of oscillation. This allows the shear rate in the fluid to be more readily optimised for the promotion of effective particle-bubble contacting.

Ni et. al. (2000) investigated the shear rate distribution in an OBC using PIV and derived the following correlations for the mean shear rate as a function of oscillatory Reynolds number and the mean energy dissipation rate: 


$$
\begin{array}{ll}
\bar{\gamma}=6 \times 10^{-4}\left(\operatorname{Re}_{o}\right)^{1.2} & (/ \mathrm{s}) \quad 251 \leq \mathrm{Re}_{o} \leq 4021 \\
\bar{\gamma}=42.6 \cdot \bar{\varepsilon}^{0.4} & (/ \mathrm{s})
\end{array}
$$

However, despite the homogeneity of the shear rates within the OBC, CFD simulations have indicated that the flow is in fact highly anisotropic with large velocity variations in the axial and radial directions compared with the tangential directions. This is an important deviation from ideal behaviour and may have implications for the OBC's efficacy as a research tool.

Chew et. al. (2004) were the first to compare the shear rate distributions in both an OBC and an impeller driven stirred tank using CFD simulation. Their simulations considered not only the spatial variation of shear rate, but also the temporal distribution of shear rates encountered by particles in the fluid. To obtain the temporal shear rate distribution, they numerically simulated the path of inert $1 \mu \mathrm{m}$ carbon particles through each system. The results indicated that for a stirred tank, particles spend the majority of their residence time in low shear bulk regions of the cell where the fluid velocities are low. When particles enter the high shear impeller zone, they were quickly ejected by the high fluid velocities produced in this zone. In contrast, particles in an OBC spent comparatively little time in the regions of lowest shear in the cell and were found to spend most of their residence time in the regions of higher shear, such as the vortices generated behind the baffles. This is an important finding as it shows that the shear distribution observed within the fluid is not necessarily the same as the shear rate distribution to which particles and bubbles may be exposed.

This finding is supported by experimental studies on particle suspension under oscillatory flow (Mackley et. al., 1993, Levesley \& Bellhouse, 1997). These studies showed that particles can in fact be retained in suspension, sometimes indefinitely, by the trapping of particles within vortices generated under oscillatory flow. Furthermore, it has been noted in studies on gas hold-up in OBC's that the oscillation through the 
orifice plates could result in bubbles being trapped within the vortices produced downstream of the baffles (Baird et. al., 1996).

This effect, known as vortex trapping, is fairly well studied and has been demonstrated experimentally for both bubbles and particles (Tooby et. al., 1977). It can be shown that particles with a particular settling velocity can be trapped indefinitely by following a fixed path within a vortex. This path is determined by the point where the local fluid velocity is equal and opposite to the particle settling velocity. This is illustrated in the Figure 2.12:

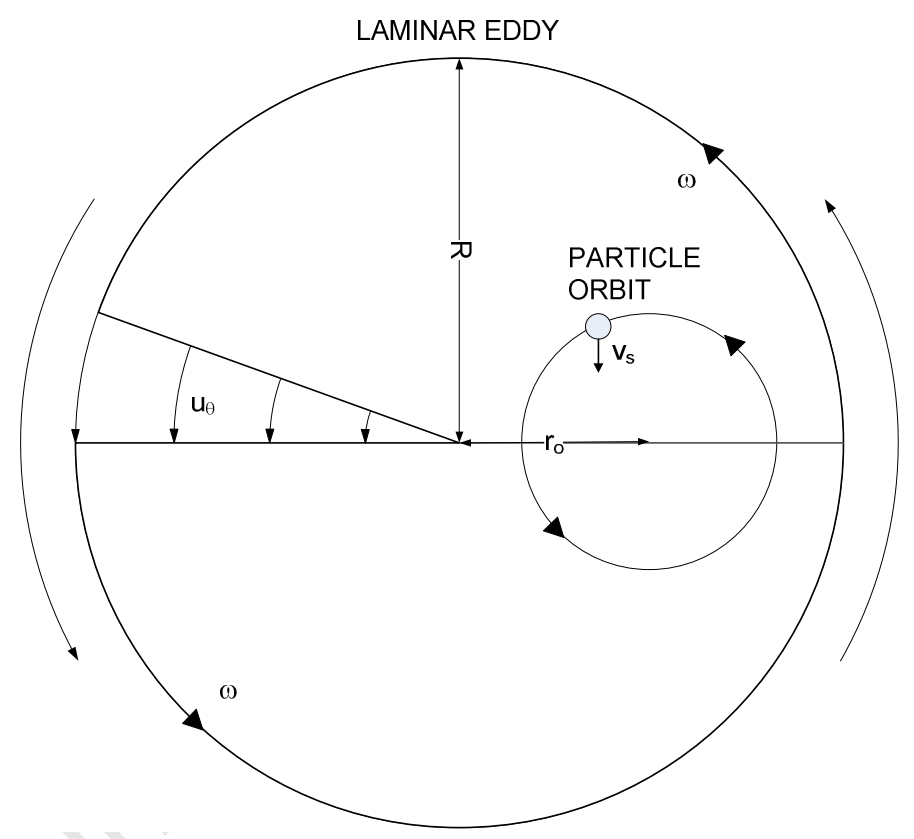

Figure 2.12 - Mechanism of Vortex Trapping in a Laminar Eddy (Tooby et al., 1977)

Figure 2.12 shows the theoretical particle orbit within a centrifugal flow field, such as a vortex under oscillatory flow. The centre of this orbit occurs at a radius given by the ratio of the terminal settling velocity $\left(\mathrm{v}_{\mathrm{s}}\right)$ and the angular velocity $(\omega)$. Thus, if the particle is neutrally buoyant, it will rotate about the centre of the eddy. The radius of the orbit path is set by the initial conditions but the particle may slowly spiral inwards or outwards over time depending on particle size and density. 
If this effect is indeed present in OBC's as indicated by a significant body of experimental studies (Mackley et al., 1993, Baird et al., 1996, Levesley \& Bellhouse, 1997), it may have an effect on the flotation behaviour of an OBC. Particles and bubbles may become trapped in high concentrations within vortices where higher shear rates are experienced. This preferential concentration of particles and bubbles would therefore have an effect on the particle-bubble contacting rate, as has been demonstrated in particle-particle collision systems (Reade \& Collins, 2000).

Interestingly, Fitch et. al. (2005) found that the flow patterns observed in an OBC are relatively unaffected by changes in the yield stress and viscosity of the fluid. This is in stark contrast to findings in conventional stirred systems which indicate the formation a "turbulent cavern" around the impeller when fluid yield stress is increased (Moore \& Cossor, 1995, Fangary et al., 2000, Arratia et al., 2006). The findings in an OBC however indicate little effect of viscosity on the mixing pattern for viscosities as high as $8 \mathrm{cP}$. It was only at a viscosity of $70 \mathrm{cP}$ that the results showed a significant damping of the vortices generated. For non-Newtonian, high yield stress fluids, Fitch et. al. (2005) approximated the fluid behaviour using an apparent viscosity fitted with the power-law index. Similar results were found, where the flow patterns were largely unaffected at a volume-averaged viscosity of $15 \mathrm{cP}$, but were significantly damped when the average viscosity was increased to $25 \mathrm{cP}$.

To quantify this effect, a velocity ratio in the cell was defined, as the ratio of axial to radial velocities. The results are illustrated in Figure 2.13. 


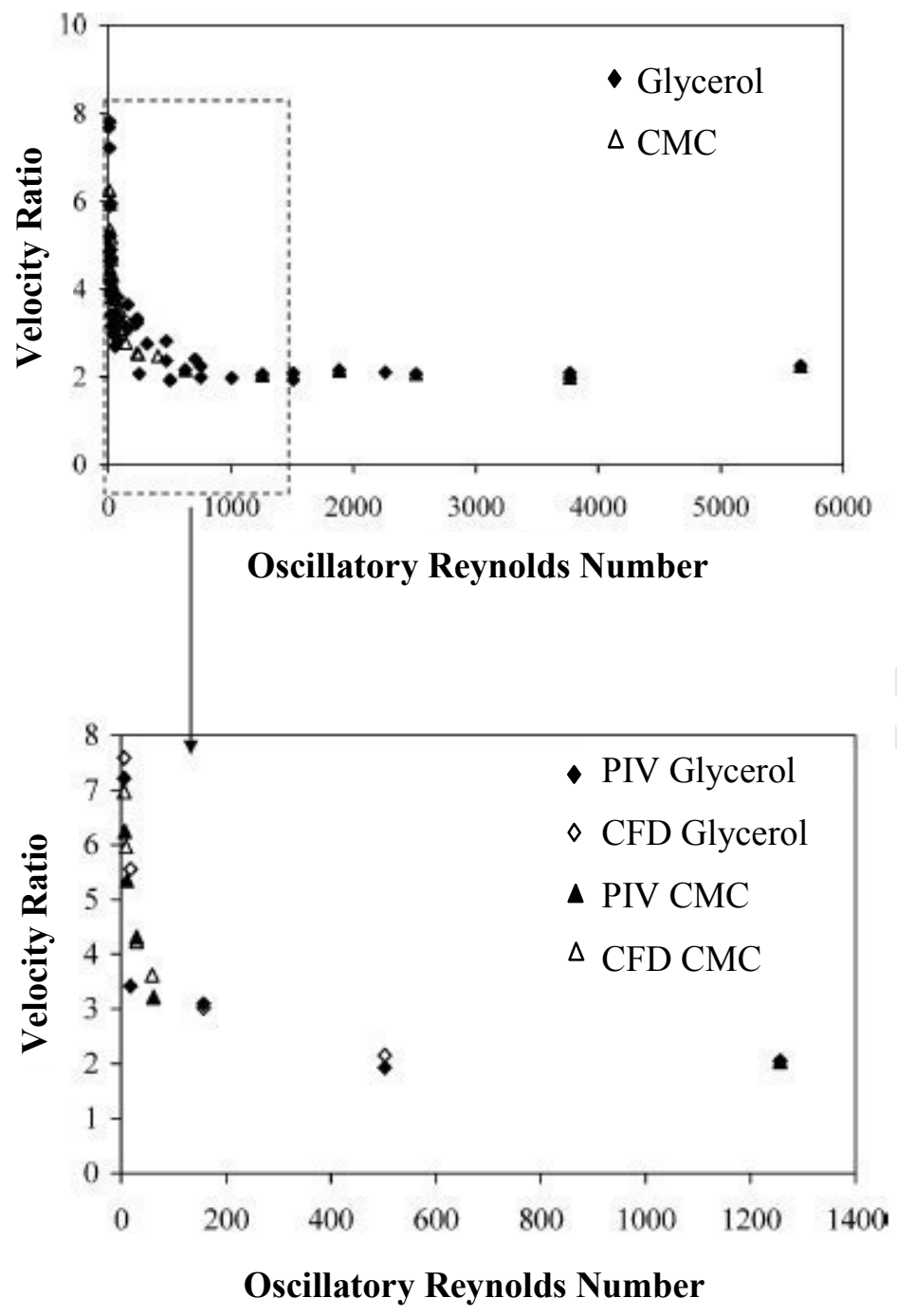

Figure 2.13 - The Effect of Apparent Viscosity on the Ratio of Axial to Radial Velocities in an OBC for a Water-Glycerol and Water-CMC Mixture (Fitch et al., 2005)

As shown in Figure 2.13, the velocity ratio was found to be constant at approximately 2 for all settings where the oscillatory Reynolds number was greater than 1000 . This indicates that the flow patterns were unaffected by the significant changes in the fluid rheology. However, as the Reynolds number decreased below 1000, the velocity ratio rose sharply to 7.5 at $\mathrm{Re}_{\mathrm{o}}=6$, indicating that the radial velocities had substantially decreased and the mixing pattern in the cell was almost completely damped out.

This independence of the flow patterns in an $\mathrm{OBC}$ to large changes in rheology for $\mathrm{Re}_{\mathrm{o}}$ $>1000$ is in stark contrast to conventional flotation cells where the flow behaviour has 
been shown to vary considerably with large changes in slurry rheology. The slurry high yield stresses have been found to lead to cavern formation around the impeller (Moore \& Cossor, 1995, Fangary et al., 2000, Arratia et al., 2006) which can have an extremely negative effect on gas dispersion (Deglon et al., 2007). The independence of the OBC's flow patterns on rheology for $\mathrm{Re}_{\mathrm{o}}>1000$ indicates that this type of cell may be effective in the flotation of rheologically complex ore types.

\subsubsection{Power Characteristics of an OBC}

The major difference between the power characteristics of an $\mathrm{OBC}$ and those of a stirred system is the oscillatory nature of the power input in an OBC. To illustrate this, let us consider an $\mathrm{OBC}$ where the baffle cage is oscillated sinusoidally through the fluid with time. For this system, one can write the following equations for the displacement, velocity and acceleration of the plate stack:

$$
\begin{aligned}
& x=-x_{o} \cdot \cos (\omega \cdot t) \\
& u=x_{o} \cdot \omega \cdot \sin (\omega \cdot t) \\
& a=x_{o} \cdot \omega^{2} \cdot \cos (\omega \cdot t)
\end{aligned}
$$

The force exerted by the oscillation of the cage must overcome both the fluid's inertia and the frictional resistance as it is agitated. This force can be measured experimentally as a pressure drop across the plate stack. This pressure drop is given by the sum of its inertial and frictional components, viz. (Jealous \& Johnson, 1955):

$$
\Delta P=\Delta P_{i}+\Delta P_{f}
$$

Here, the pressure drop due to inertia is a function of the fluid mass multiplied by the acceleration of the baffle cage. The pressure drop due to friction is proportional to the velocity of the baffle cage squared. The resulting pressure wave therefore takes the form of the following equation: 
$\Delta P=\Delta P_{i, \max } \cdot \cos (\omega \cdot t)+\Delta P_{f, \text { max }} \cdot \sin (\omega \cdot t) \cdot|\sin (\omega \cdot t)|$

where $\Delta \mathrm{P}_{\mathrm{i}, \max }$ and $\Delta \mathrm{P}_{\mathrm{f}, \max }$ are the maximum inertial and frictional pressure drops respectively. The instantaneous power input to the $\mathrm{OBC}$ is then given by the product of this pressure drop, the velocity of the baffle cage and the cross sectional area of the column (Hafez \& Prochazka, 1974):

$J=\left(\frac{\pi \cdot D^{2}}{4}\right) \cdot \Delta P \cdot v_{\text {cage }}$

where $\mathrm{J}$ is the instantaneous power input to the fluid, $\Delta \mathrm{P}$ is the pressure drop across the baffle cage and $v_{\text {cage }}$ is the velocity of the baffle cage. Combining these equations, one obtains the following equation for the instantaneous power input to the system:

$J=\frac{\pi D^{2}}{4} \cdot\left(\Delta P_{i, \max } \cdot x_{o} \cdot \omega \cdot \cos (\omega \cdot t) \cdot \sin (\omega \cdot t)+\Delta P_{f, \max } \cdot x_{o} \cdot \omega \cdot|\sin (\omega \cdot t)|^{3}\right)$

Since the power oscillates with time, the time-averaged power dissipation is then given by averaging the power over each cycle of oscillation:

$J_{a v e}=\frac{\int_{0}^{T} J \cdot d t}{\int_{0}^{T} d t}$

where $\mathrm{T}$ is the period of oscillation.

Thus, the instantaneous power input to an $\mathrm{OBC}$ is cyclic and is modelled as the sum of two individual components; inertia and friction. In a conventional stirred system, only 
the frictional component is present since the system operates at steady state. The relationship of these two components in an OBC is illustrated in the Figure 2.14:

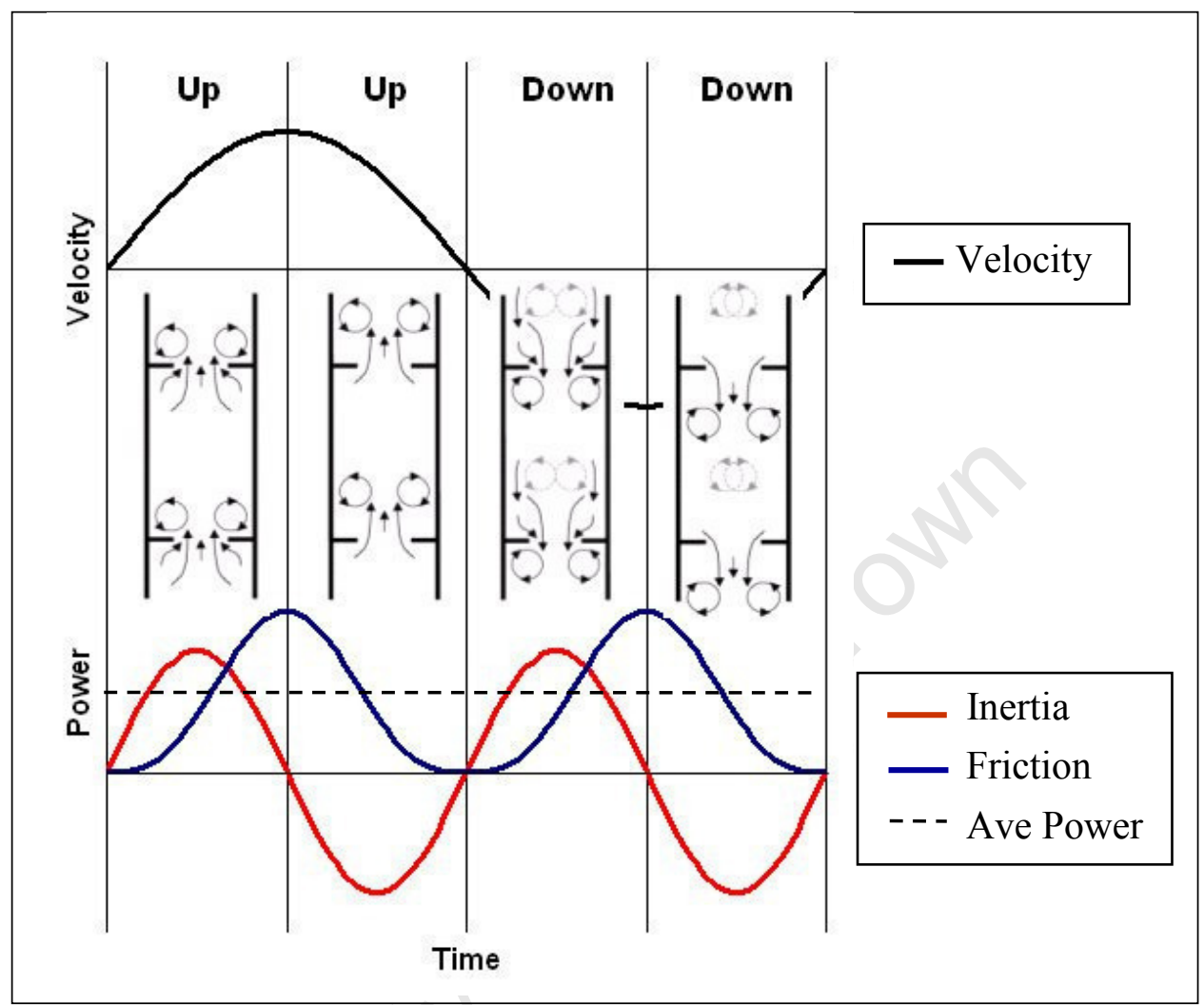

Figure 2.14 - Schematic of the Components of Instantaneous Power Input to an OBC

At the start of the upward stroke in an $\mathrm{OBC}$, power is required to accelerate the fluid through the baffle constriction. The inertial power requirement therefore increases initially with this acceleration but decreases to zero as the fluid reaches its maximum velocity. At this point, the fluid possesses sufficient inertia to continue on its upward path without added energy input and the kinetic energy of the fluid is recovered as the fluid decelerates. The same is true on the downward stroke, where energy is required to accelerate the fluid inertia to its maximum velocity, before being recovered as the fluid decelerates in the final part of the cycle. The time-averaged power required to sustain this oscillation in inertia is zero since the fluid does not undergo any net acceleration. 
In contrast, the power required to overcome fluid friction remains positive throughout the cycle and is proportional to the cube of fluid velocity. Note that since the average power input required to maintain the oscillation in fluid inertia is zero, the timeaveraged power input to the cell is affected only by the frictional component of power input. This is logical since only the friction in the fluid gives rise to the dissipation of energy in the system as heat.

This inertial component of the power input therefore gives rise to the significant oscillatory motion of the fluid but does not contribute to the overall power dissipation in the system. The OBC therefore produces significantly more fluid movement per unit power dissipation than would be obtained in a non-oscillatory system.

Over the years, researchers have derived various models to account for the inertial and frictional contributions to the instantaneous power input. The following section presents a brief summary of the two most commonly used models; the quasi-steady state model and the acoustic model.

\subsubsection{The Quasi-Steady State Model}

The first attempt to model power consumption in an oscillatory flow system was made by Jealous \& Johnson (1955). They derived equations for instantaneous pressure and power input as a function of time and compared their results with experimental data.

For the inertial pressure drop they assumed no interference between the fluid and the adjacent plates, meaning that the inertial pressure drop is given simply by the fluid mass multiplied by the acceleration.

$$
\Delta P_{i}=\rho \cdot Z \cdot x_{o} \cdot \omega^{2} \cos (\omega \cdot t)
$$

where $\mathrm{Z}$ is the height of fluid being pulsed in the system. 
In order to derive an equation for frictional pressure drop, they assumed that the instantaneous frictional pressure drop under oscillatory flow is equal to the frictional pressure drop produced under steady state conditions (quasi steady state assumption). This enabled them to use the well known correlation for frictional pressure drop through an orifice plate at steady state:

$$
\Delta P_{f}=\frac{\rho \cdot u^{2}}{2} \cdot \frac{\left(1-S^{2}\right)}{C_{o}^{2} \cdot S^{2}}
$$

where $\mathrm{C}_{\mathrm{o}}$ is the drag coefficient for an orifice plate under steady state conditions, which is usually taken as 0.6 but can be determined more accurately experimentally. By substituting in an equation for the fluid velocity in an oscillatory flow system, they obtained:

$$
\Delta P_{f}=\frac{n \cdot \rho \cdot\left(1-S^{2}\right)}{2 \cdot C_{o}^{2} \cdot S^{2}} \cdot x_{o}^{2} \cdot \omega^{2} \cdot|\sin (\omega \cdot t)| \cdot \sin (\omega \cdot t)
$$

The instantaneous power input to the system is then given by substituting these equations into equation 2.13 to give:

$$
J_{\text {quasi SS }}=\frac{\pi \cdot D^{2}}{4} \cdot\left(\rho \cdot Z \cdot x_{o}^{2} \cdot \omega^{3} \cdot \cos (\omega \cdot t) \cdot \sin (\omega \cdot t)+\frac{n \cdot \rho \cdot\left(1-S^{2}\right)}{2 \cdot C_{o}^{2} \cdot S^{2}} \cdot x_{o}^{3} \cdot \omega^{3} \cdot\left|\sin ^{3}(\omega \cdot t)\right|\right)
$$

The two terms on the right hand side of equation 2.19 represent the inertial and frictional contributions of power respectively. This model became known as the "quasisteady state" model. 
Now, the time-averaged power dissipation is given by integrating the above equation with respect to time. Note that on integration, the inertial term reduces to zero and the average power input is given by:

$$
\bar{J}_{\text {quasi SS }}=\left(\frac{1}{6}\right) \cdot \frac{n \cdot \rho \cdot D^{2} \cdot\left(1-S^{2}\right)}{C_{o}^{2} \cdot S^{2}} \cdot x_{o}^{3} \cdot \omega^{3}
$$

Thus, the time-average power input is only a function of frictional contribution of the power input and is not affected by the fluid inertia (c.f. Figure 2.14).

\subsubsection{The Acoustic Model}

More recently, Baird \& Stonestreet (1995) derived new expressions for inertial and frictional pressure drop under oscillatory flow and compared their results with experimental data. Their experiments focused on higher frequencies (up to $14 \mathrm{~Hz}$ ) than in previous work. They considered inertia in an OBC to be equivalent to oscillating a central plug of fluid, with length $\mathrm{Z}$ and diameter $\mathrm{D}_{\mathrm{o}}$. They then added a fitted parameter $\mathrm{K}_{\mathrm{i}}$ to account for interference between the baffles:

$\Delta P_{i}=\rho \cdot\left(\frac{Z \cdot K_{i}}{S}\right) \cdot x_{o} \cdot \omega^{2} \cdot \cos (\omega \cdot t)$

Frictional pressure drop was derived from the acoustic resistance of a single thin orifice. This is given by (Panton \& Goldman, 1976):

$$
\Delta P_{f}=3 \cdot \rho \cdot(v \cdot \omega)^{0.5} \cdot u
$$

where $u$ refers to the velocity through the orifice itself, given by $x_{0} \cdot \omega \cdot \sin (\omega \cdot t) / S$. This equation was found to apply at low sound intensity (amplitude) and high frequency (i.e. 
when $\left.\mathrm{u} /(v \cdot \omega)^{0.5}<3\right)$. The kinematic viscosity in equation 2.22 was replaced with the larger turbulent eddy viscosity, given by:

$v_{e}=l^{2} \cdot \omega$

where $l$ is the mixing length corresponding to the average distance travelled by turbulent eddies.

Combining equations 2.22 and 2.23 , the following equation is obtained:

$\Delta P_{f}=\frac{3 \cdot n \cdot \rho \cdot l}{S} \cdot x_{o} \cdot \omega^{2} \cdot \sin (\omega \cdot t) \cdot|\sin (\omega \cdot t)|$

The mixing length, $l$, is not known a priori and must be fitted to experimental data. This parameter is expected to be of the same order as tube diameter but is believed to be a function of the cell and baffle geometry. For this reason, the same value of mixing length was used for fitting of all the pressure responses. It is speculated however that if mixing length is indeed a measure of the average distance propagated by turbulent eddies, it may be more appropriate to fit the mixing length as a function of amplitude since amplitude controls the distance which eddies are propagated into the inter-baffle cavities (Fitch, 2003).

Thus Baird \& Stonestreet's model consists of 2 adjustable parameters; one for fluid inertia and one for friction. This model became known as the "acoustic model" due to its derivation from the acoustic resistance of orifice plates. It was found that this model fitted well for low amplitudes and high frequencies ( 1 to $5 \mathrm{~mm}$ and 3 to $14 \mathrm{~Hz}$ ), while the quasi-steady state model remained applicable for large amplitude and low frequency combinations ( 5 to $30 \mathrm{~mm}, 0.5$ to $2 \mathrm{~Hz}$ ). 
Once again, instantaneous power dissipation is the product of the instantaneous pressure drop across the baffle cage and the velocity:

$$
J_{\text {acoustic }}=\frac{\pi \cdot D^{2}}{4}\left(\rho \cdot\left(\frac{Z \cdot K_{i}}{S}\right) \cdot x_{o}^{2} \cdot \omega^{3} \cdot \cos (\omega \cdot t) \cdot \sin (\omega \cdot t)+\frac{3 \cdot n \cdot \rho \cdot l}{S} \cdot x_{o}^{2} \cdot \omega^{3} \cdot\left|\sin ^{3}(\omega \cdot t)\right|\right)
$$

The time-averaged power dissipation is then given by:

$$
\bar{J}_{\text {acoustic }}=\frac{\rho \cdot n \cdot D^{2} \cdot l \cdot x_{o}^{2} \cdot \omega^{3}}{S}
$$

The above formula is in contradiction to the formula quoted by Baird et. al. (1996), who added a coefficient of $(3 \pi / 8)$. This coefficient is believed to arise from an error in the integration of the sine cubed term in equation 2.25. This mistake would be difficult to notice however since any error in the coefficient would be taken up by the fitting of the mixing length parameter. However if one first fits the pressure signal and then calculates the power input, the error becomes apparent. The full derivation of equation 2.26 is given in Appendix D.

Thus power dissipation in an OBC consists of two different regimes; a "quasi-steady state" regime at high amplitude, in which friction is well approximated by the steady state frictional pressure drop, and an "acoustic" regime at low amplitudes where friction is approximated by the frictional resistance of sound waves.

Also important to note is that power input in an $\mathrm{OBC}$ is the sum of 2 components; an inertial component which accelerates and decelerates the fluid, and a frictional component which dissipates energy. On a time-averaged basis, only the frictional energy loss contributes to the total power input. 


\subsubsection{Analysis of the $\mathrm{OBC}$ as a Novel Cell}

The preceding section has reviewed the numerous attributes of the $\mathrm{OBC}$ design which have made it effective in other industrial applications. Table 2.3 summarises the potential strengths and weaknesses of the $\mathrm{OBC}$ in application as a novel flotation device for fine particle recovery. This analysis is performed using the SWOT analysis technique detailed in section 2.1.4 and may be compared with the SWOT analysis of other cell types which is shown in Table 2.1. 
Table 2.3: SWOT Analysis of the Application of an OBC as a Novel Flotation Cell for Fine Particle Recovery

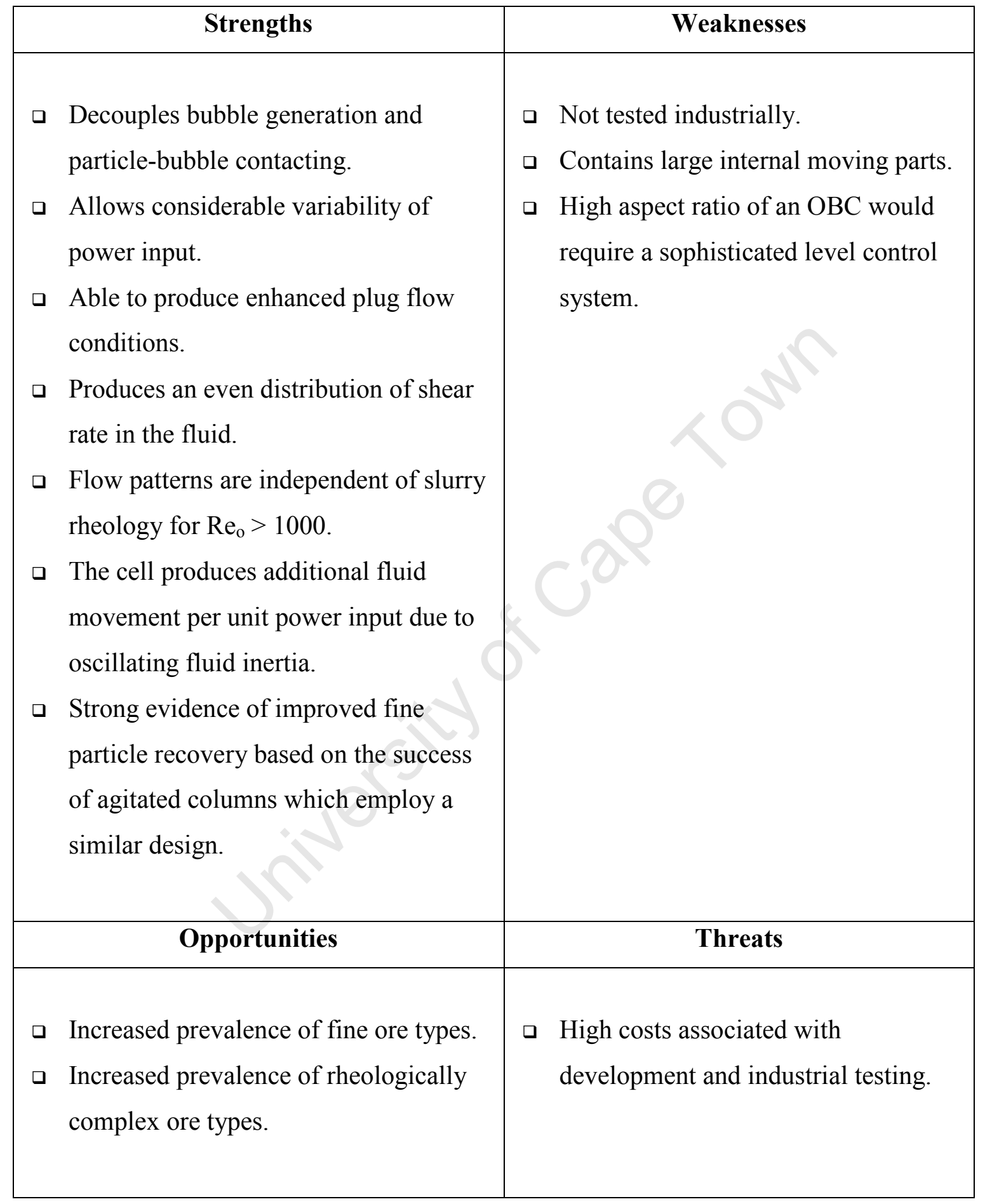


Table 2.3 indicates that the $\mathrm{OBC}$ has a number of attributes which would be highly advantageous if it were applied as a novel cell for fine particle recovery. The cell's ability to decouple the bubble generation and particle-bubble contacting functions of the cell, together with the enhanced variability of power input, makes it an ideal environment in which to optimise the hydrodynamic conditions for the recovery of fine particles. The unique agitation mechanism of the $\mathrm{OBC}$ also produces a more even distribution of shear rate in the fluid and flow patterns which are largely independent of slurry rheology for $\mathrm{Re}_{\mathrm{o}}>1000$. This strongly suggests that the cell may have considerable potential in the treatment of rheologically complex ore types.

Furthermore, as noted in Chapter 1, the OBC's unique hydrodynamic environment provides an interesting opportunity to investigate the role which hydrodynamics play in promoting particle-bubble contacting. The following section details the current understanding of the role of hydrodynamics in determining particle-bubble contacting and examines both the fundamental models which have been developed and the various experimental studies which have been conducted. The section ends with a summary of the effect of the key variables which affect the flotation rate and a SWOT analysis of the $\mathrm{OBC}$ as research tool for the investigation of the effect of hydrodynamics on particle-bubble contacting.

\subsection{The Effect of Hydrodynamics on Particle-Bubble Contacting}

Due to the complex nature of fluid mechanics, the role which hydrodynamics play in particle-bubble contacting in turbulent systems has largely been restricted to the development of fundamental models. These models are usually based on the statistical description of turbulence developed by Kolmogorov (1941). Experimental validation of these models has been extremely difficult since their application requires a detailed knowledge of the fluid flow environment. For this reason, much work is being conducted into the development of CFD models of flotation cells. It is hoped that the incorporation of fundamental flotation models into a CFD simulation environment will provide a powerful tool for optimising flotation cell hydrodynamics. However the development of such a simulation is no trivial task since few of the fundamental models 
have been experimentally validated. The advent of this type of simulation therefore seems to be some years off, although significant advances have been reported in the literature in recent years (Koh \& Schwarz, 2003, Koh \& Schwarz, 2007).

To date, most of the experimental investigations into hydrodynamics and particlebubble contacting have been conducted in stirred tank environments, which provide a particularly uneven distribution of energy dissipation rates. While much useful information has been gained, these studies are extremely limited in their ability to attribute improvements to contacting in the bulk of the cell or improvements in the impeller zone. This makes extrapolation of the results to larger systems very difficult.

The following sections will review the current understanding of role which hydrodynamics play in promoting particle-bubble contacting in order to provide a basis for the interpretation of flotation data in the OBC. In this review, emphasis is given to contacting in turbulent systems and quiescent systems are not considered.

\subsubsection{Fundamental Modelling}

\subsubsection{The Statistical Description of Turbulence}

The mechanical agitation of fluid in a flotation cell results in the transfer of kinetic energy from the agitation device to the fluid. This kinetic energy results in the formation of large scale eddies in the fluid. The overall amount of energy contained in the system is balanced by a frictional dissipation of energy by viscous forces.

The statistical description of turbulence describes the mechanism by which the fluid kinetic energy is dissipated by friction. The model was first derived by Kolmogorov (1941) and is well documented in the literature. The following overview is derived from Hinze (1959), Deglon (1998) and Davidson (2003).

The statistical description of turbulence considers turbulence to consist of a spectrum of differently sized eddies. Energy is continually transferred from the larger eddies to the 
smaller ones through turbulent shear stress and it is these smaller eddies that eventually dissipate all the energy as heat. The distribution of kinetic energy contained within each eddy size is known as the turbulent kinetic energy spectrum, and is shown schematically in the following figure:

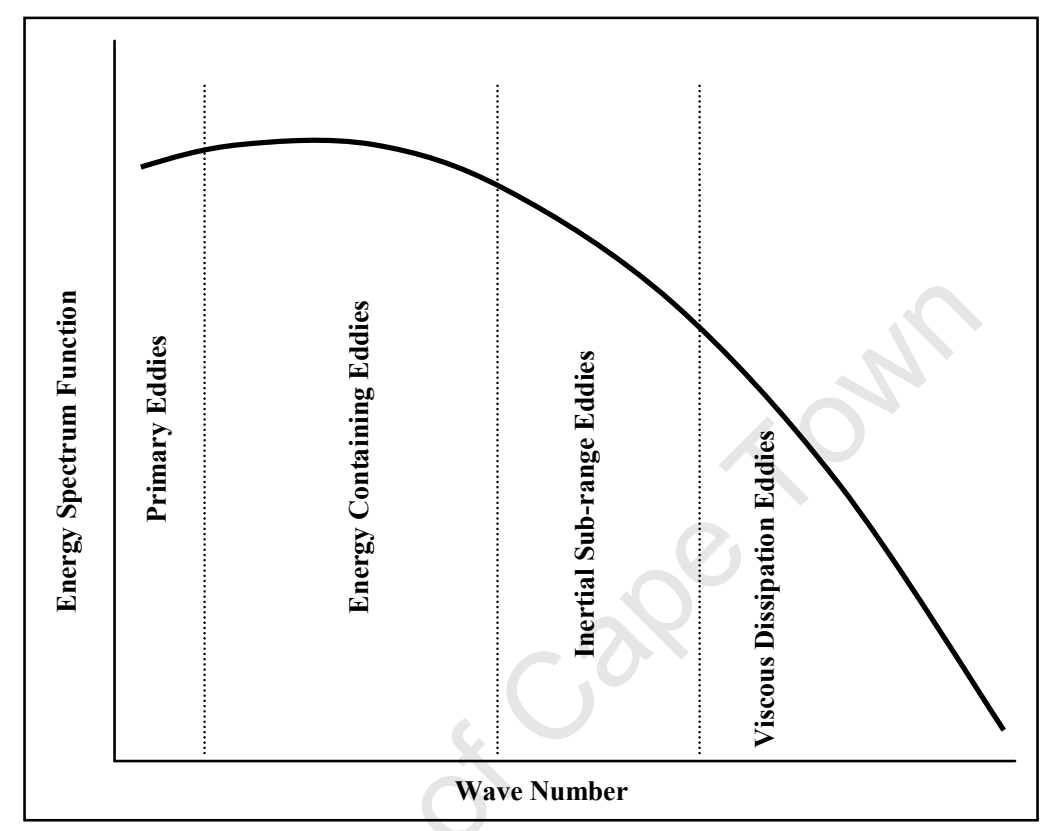

Figure 2.15 - Schematic Representation of a Turbulent Kinetic Energy Spectrum (from Deglon, 1998)

Eddy size is inversely proportional to eddy wave number, and thus the largest eddies occur on the extreme left of the energy spectrum. These eddies are generally known as the "primary eddies" and are thought to be of similar scale to the cell geometry. These eddies then transfer their energy to smaller eddies known as the "energy containing eddies" which contain the bulk of the turbulent kinetic energy in the fluid. As eddy size decreases further, the amount of energy transferred becomes large compared to the rate of change of energy within each eddy. The energy contained in these eddies therefore remains approximately constant regardless of the bulk fluid motion. These eddies are known as the "inertial sub-range eddies" and are defined as being small enough for their energy to be statistically independent of the bulk flow, but large enough to transfer a 
significant amount of their energy via inertial forces. These eddies require a large total kinetic energy to form and are therefore only present in fully turbulent flow. The energy from these eddies is then transferred to the smallest class of eddies, known as the "viscous dissipation eddies", in which the bulk of system's energy is dissipated as heat. These eddies are small enough that the viscous forces in the fluid dominate and the flow within them is essentially laminar.

The scale of these smallest eddies is dependent on both the rate of energy being dissipated within them and the viscous forces responsible for the dissipation. Kolmogorov (1941) derived the following equations to characterise the length and time scales on which dissipation occurs:

$$
\begin{aligned}
& \eta=\left(\frac{v^{3}}{\varepsilon}\right)^{1 / 4} \\
& \tau_{k}=\left(\frac{v}{\varepsilon}\right)^{1 / 2}
\end{aligned}
$$

These scales are known as the Kolmogorov length scale $(\eta)$ and Kolmogorov time scale $\left(\tau_{\mathrm{k}}\right)$ and are functions of the kinematic viscosity of the fluid $(v)$ and the energy dissipation rate $(\varepsilon)$. Eddies with dimensions $r_{e} \cong(4$ to 6$) \eta$ are thought to be laminar and eddies with $r_{e} \geq(12$ to 15$) \eta$ are thought to be turbulent.

The fluid velocity under turbulent conditions is often characterised as having a mean and fluctuating component. The fluctuating component is the result of the action of all eddies within the turbulent kinetic energy spectrum.

$U=\bar{U}+U^{\prime}$ 
The fluctuating velocity therefore provides a useful way of characterising the turbulent kinetic energy spectrum. For this reason, the turbulent kinetic energy is often estimated as the sum of the kinetic energy of the three fluctuating velocity components, viz.

$$
T K E=\frac{1}{2} \cdot\left(\overline{U_{x}^{\prime 2}}+\overline{U_{y}^{\prime 2}}+\overline{U_{z}^{\prime 2}}\right)
$$

For isotropic turbulence, the fluctuating components of velocity are considered to be approximately equal in all directions and thus the above equation reduces to:

$T K E \cong \frac{3}{2} \cdot \overline{U^{\prime 2}} \quad$ (isotropic turbulence)

Thus, for isotropic turbulence, the turbulent characteristics of the flow can be described by the energy dissipation rate $(\varepsilon)$, the mean square fluctuating velocity $\left(\overline{U^{\prime 2}}\right)$ and the kinematic viscosity of the fluid $(v)$.

It is on this assumption of isotropy that most of the turbulent collision models are based. The following section will review the major developments in the use of turbulence theory to predict collision and detachment rates under turbulent flow conditions.

\subsubsection{Particle-Bubble Collision under Turbulent Conditions}

Turbulent collision has been a subject of interest in numerous research fields from the aggregation of solid particles, to the coalescence of droplets and bubbles, to the collision of particles and bubbles in flotation. In most cases, the models have been formulated on a volumetric basis, based on the work of von Smoluchowski (1917) who derived equations for particle-particle collisions using a population balance approach. In flotation applications, the models have generally taken the following form (Schulze, 1993): 
$\frac{d N_{p}}{d t}=-k \cdot N_{P}=-Z_{p b} \cdot N_{b} \cdot N_{p} \cdot P_{c} \cdot P_{a} \cdot\left(1-P_{d}\right)$

Here $N_{p}$ and $N_{b}$ refer to the number of particles and bubbles respectively. $P_{c}, P_{a}$ and $P_{d}$ are the probabilities of collision, attachment and detachment respectively. These probabilities account for the probability of a particle successfully intercepting the bubble and attaching to it, without becoming destabilised by detaching forces. $Z_{\mathrm{pb}}$ refers to number of collisions per unit volume and time, which is generally given by a complex function of particle size, bubble size, energy dissipation rate, the mean square fluctuating velocity and the kinematic viscosity of the fluid. The derivation of the collision frequency is dependent on the mode of collision, which can occur by the mechanisms shown in Table 2.4. 
Table 2.4: Collision Mechanisms in Turbulence

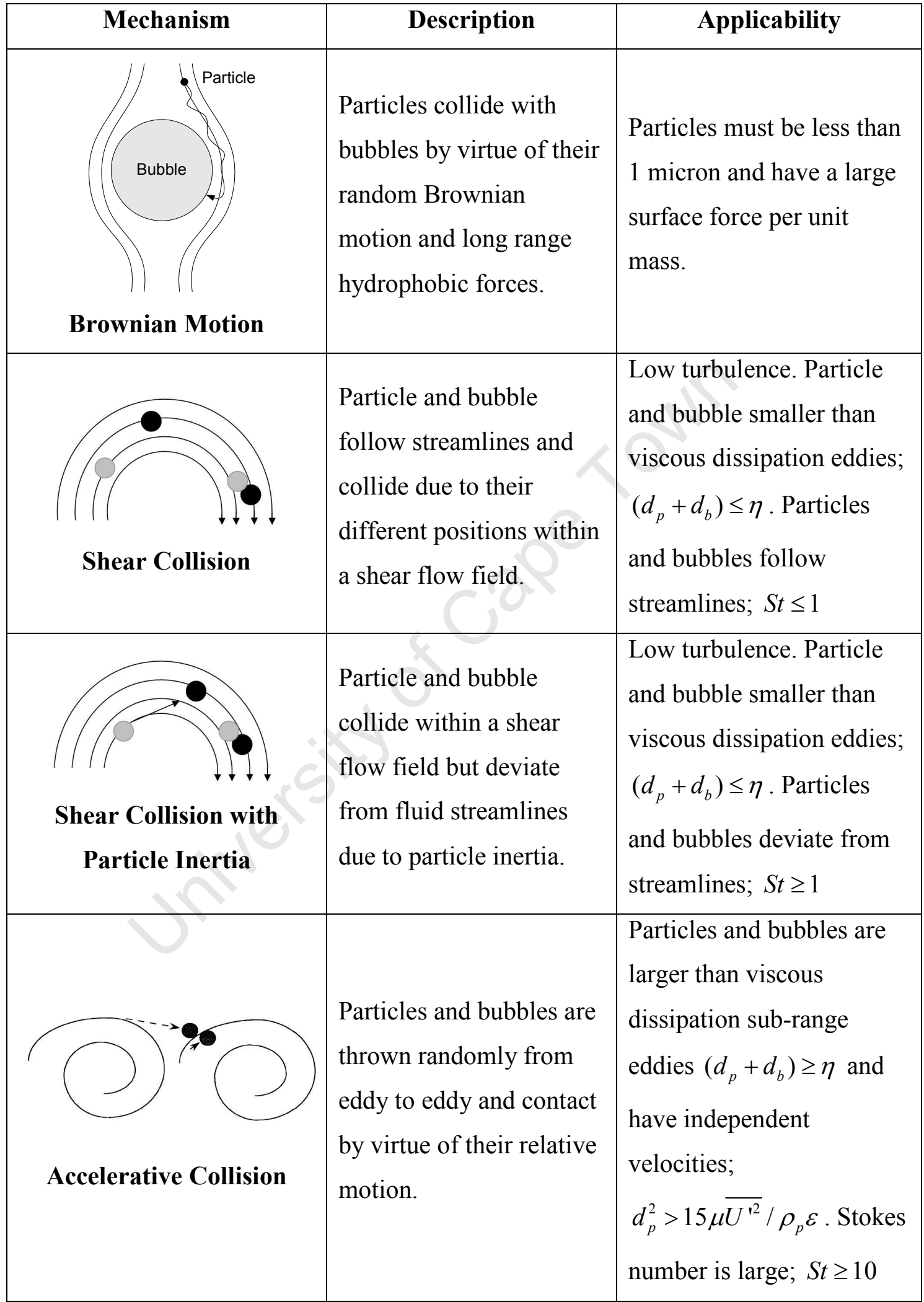


Brownian motion is considered to only have an effect on particle-bubble contacting for particles of less than 1 micron. This is considerably less than the range of particles sizes usually encountered in most flotation applications and is therefore only of limited interest in flotation research. For this reason, the development of models to describe collision by Brownian motion has been largely limited to particle-particle collisions, where the calculation of collision frequency is generally based on Fick's Law of diffusion. The diffusion coefficient of the particle is given by the Stokes-Einstein equation, viz.

$D_{i}=\frac{\kappa_{B} \cdot T}{6 \pi \cdot \mu \cdot r_{p}}$

where $D_{i}$ is the diffusivity of particle size class $i$ due to Brownian motion. $\kappa_{B}$ is the Boltzmann constant, $\mathrm{T}$ is the absolute temperature and $\mu$ is the fluid viscosity. The resulting collision frequency between two bodies colliding due to their Brownian motion is therefore given by (Smoluchowski, 1917):

$Z_{i j}=\frac{2 \cdot \kappa_{B} \cdot T}{3 \cdot \mu} \cdot\left(\frac{1}{r_{i}}+\frac{1}{r_{j}}\right)\left(r_{i}+r_{j}\right)$

Recently, Nguyen et. al. (2006) investigated factors affecting the flotation of submicron particles, and derived equations to describe the effect of Brownian motion and surface forces - such as van der Waals forces, electric double layer forces and hydrophobic forces - on the particle collection efficiency. Both the experimental and theoretical results indicated that particles exhibit a minimum collection efficiency at a particle size of approximately $100 \mathrm{~nm}$. Above this particle size, the collection efficiency was dominated by particle-bubble interception effects but increased significantly below this size due to the increased influence of Brownian motion and colloidal forces. The study therefore suggests that the effect of Brownian motion is only significant for particles less than approximately $100 \mathrm{~nm}$ and is therefore only of limited interest in most industrial flotation applications. 
The major collision mechanisms of interest in flotation are therefore the shear and accelerative collision mechanisms. For these mechanisms, the mode of collision is dependent on eddy size and the tendency of particles and bubbles to follow streamlines in the fluid.

The tendency of particles to follow streamlines is characterised by the Stokes number (St), defined as the ratio of particle relaxation time to Kolmogorov timescale, viz.

$$
S t=\frac{\tau_{i}}{\tau_{k}}=\frac{\tau_{i}}{\sqrt{v / \varepsilon}}
$$

Here, the particle relaxation time $\left(\tau_{\mathrm{i}}\right)$ is defined as the time for a particle to slow down from $\mathrm{V}_{0}$ to $\mathrm{V}_{0} / \mathrm{e}$ if the particle is projected at a velocity $\mathrm{V}_{0}$ into a stagnant fluid. Relaxation time can be calculated by the following equation:

$$
\tau_{i}=\frac{C_{c, i} \cdot\left(2 \rho_{i}+\rho_{f}\right) \cdot r_{i}^{2}}{9 \mu}
$$

where $\mathrm{C}_{\mathrm{c}, \mathrm{i}}$ is the Cunningham slip correction factor, equal to 1 for liquid systems (Kruis \& Kusters, 1997). If St $\square$ 1, particles will follow fluid streamlines but if $S t \square 1$, particles will be relatively unaffected by fluid motion. The following figures show the range of Stokes numbers of typical particles and bubbles in flotation. 


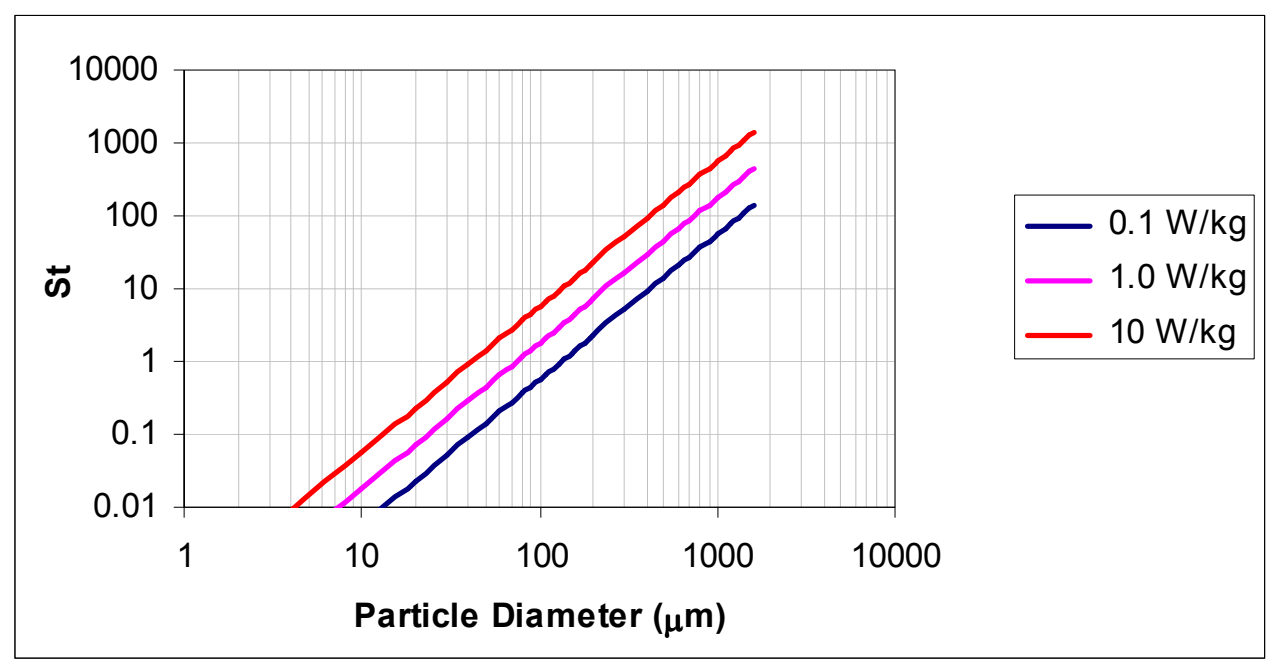

Figure 2.16 - Stokes Number as a Function of Particle Diameter and Energy Dissipation Rate for Quartz Particles in Water $\left(\rho_{s}=2700 \mathrm{~kg} / \mathrm{m}^{3}, \rho_{\mathrm{f}}=1000 \mathrm{~kg} / \mathrm{m}^{3}, \mu=1 \mathrm{cP}\right)$

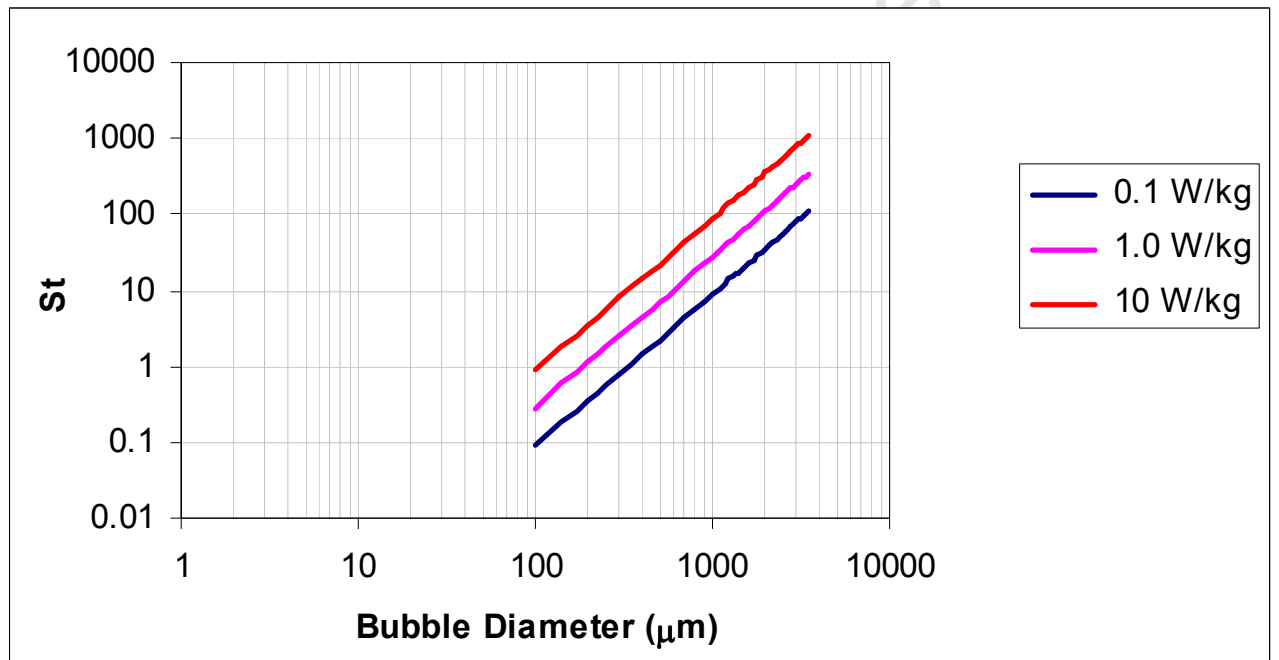

Figure 2.17 - Stokes Number as a Function of Bubble Diameter and Energy Dissipation Rate for Air Bubbles in Water $\left(\rho_{\mathrm{f}}=1000 \mathrm{~kg} / \mathrm{m}^{3}, \mu=1 \mathrm{cP}\right)$

Figures 2.15 and 2.16 illustrate that for a typical flotation energy dissipation rate of 1 $\mathrm{W} / \mathrm{kg}$, particles smaller than 75 micron have $S t \leq 1$, and therefore follow the fluid streamlines, while bubbles greater than 200 micron have $S t \geq 1$ and therefore deviate from fluid streamlines. This tendency to either follow fluid streamlines or deviate from them largely determines the collision mechanism which applies. 
For particles and bubbles with $S t \square$ 1, particles follow streamlines within the viscous dissipation sub-range eddies and the shear collision mechanism is thought to apply. As the particle relaxation time becomes more significant and Stokes number increases, particles start to deviate from fluid streamlines and contacting rates increase. As the Stokes number is further increased, particles and bubbles are no longer contained within the viscous eddies and are thrown randomly from eddy to eddy before colliding. Under these conditions, the accelerative mechanism is thought to apply.

Von Smoluchowski (1917) was the first to develop a collision model for particleparticle collision in shear flow field.

$$
Z_{\text {shear }}=\frac{4}{3} \cdot\left(d_{i}+d_{j}\right)^{3} \cdot\left(\frac{d U}{d y}\right)
$$

where $\mathrm{dU} / \mathrm{dy}$ is the velocity gradient or shear rate in a laminar flow field. This equation assumes that particles follow streamlines and contact by virtue of the differences in their relative positions within the flow field. Camp \& Stein (1943) later built on this model by substituting the velocity gradient with the mean shear rate within a viscous dissipation sub-range eddy, given by the inverse of the Kolmogorov timescale. This yielded the following equation:

$$
Z_{\text {shear }}=\frac{4}{3} \cdot\left(d_{i}+d_{j}\right)^{3} \cdot\left(\frac{\varepsilon}{v}\right)^{1 / 2}
$$

Saffman \& Turner (1956) re-derived the Smoluchowski equation for turbulent conditions and added a second inertial term which accounts for the relative motion between particles and the suspending fluid, and thus made the equation applicable to particles and bubbles with $S t \geq 1$ : 
$Z_{\text {shear }+ \text { inertia }}=\left(\frac{8 \pi}{3}\right)^{1 / 2} \cdot\left(r_{i}+r_{j}\right)^{2} \cdot\left[3 \cdot\left(1-\frac{\rho_{p}}{\rho_{f}}\right)^{2} \cdot\left(\tau_{i}-\tau_{j}\right)^{2} \cdot \overline{\left(\frac{D U}{D t}\right)^{2}}+\frac{1}{3} \cdot\left(r_{i}+r_{j}\right)^{2} \cdot \frac{\varepsilon}{v}\right]^{1 / 2}$

where $\overline{(D U / D t)^{2}}$ is the average acceleration of eddies in the viscous dissipation subrange, given by:

$\overline{(D U / D t)^{2}}=1.16 \cdot \varepsilon^{3 / 2} \cdot v^{-1 / 2} \quad$ (viscous dissipation sub-range)

Note that for the above equation to apply, particles and bubbles must be contained with the viscous dissipation sub-range eddies. This equation is therefore applicable to moderately turbulent flow where the viscous dissipation sub-range eddies are large compared with the particle and bubble size. If one assumes no particle inertial effects (i.e. $S t \square 1$ ), equation 2.39 reduces to:

$Z_{\text {shear }}=\sqrt{\frac{8 \pi}{15}} \cdot\left(d_{i}+d_{j}\right)^{3} \cdot\left(\frac{\varepsilon}{v}\right)^{1 / 2}$

Abrahamson (1975) was the first to consider collision processes in vigorously turbulent fluids. Under these conditions, the viscous dissipation eddies become too small to contain particles and bubbles. Rather particles are randomly catapulted between turbulent eddies such that their velocities are completely independent of one another. He derived the following equation for such conditions, based of the elementary kinetic theory of gases:

$$
Z_{\text {accelerative }}=\sqrt{8 \pi} \cdot\left(r_{i}+r_{j}\right)^{2} \cdot \sqrt{\left(\overline{U_{i}^{\prime 2}}+\overline{U_{j}^{\prime 2}}\right)} \quad \text { (for } S t \square 1 \text { ) }
$$

This model describes the motion of the particle and bubble as having normally distributed fluctuating velocities for which the variance is given by the mean squared 
fluctuating velocity $\left(\overline{U_{i}^{\prime 2}}\right)$. This assumption of a Gaussian distribution of velocity is only valid in the inertial sub-range of turbulence (Bloom \& Heindel, 2002).

The application of this model relies on the independence of the particle and bubble velocity. In order for this to be true, the following condition must be satisfied (Abrahamson, 1975):

$d_{p}^{2}>15 \mu \cdot \overline{U^{\prime 2}} / \rho_{p} \cdot \varepsilon$

For a typical flotation cell, where the viscosity is approximately that of water $\left(1 \mathrm{x} 10^{-3}\right.$ Pa.s), particle density is $2700 \mathrm{~kg} / \mathrm{m}^{3}$, the mean squared fluctuating velocity is approximately $0.25 \mathrm{~m}^{2} / \mathrm{s}^{2}$ and the energy dissipation rate is of the order of $3 \mathrm{~W} / \mathrm{kg}$, this equates to a minimum particle size of 680 microns. This is well out of range of the typical flotation size, and in fact, it would take an energy dissipation rate of greater than $100 \mathrm{~W} / \mathrm{kg}$, to bring this figure to within range of typical flotation particles. Furthermore, the model assumes an infinite particle Stokes number. While this gives a reasonable approximation for $\mathrm{St}>10$, the equation tends to over predict collision rates for $\mathrm{St}<10$ (Sherrel, 2004). Thus the use of the Abrahamson collision model is not strictly valid in flotation applications.

Despite these difficulties in meeting the constraints of the Abrahamson model, it has been used extensively to predict collision rates in turbulent flotation systems (Schubert, 1999, Bloom \& Heindel, 2002, Pyke et. al., 2003, Sherrel, 2004, Ralston et. al., 2005). Bloom \& Heindel (2002) however argued that if the bubble rise velocity is included in the calculation of the fluctuating velocity in equation 2.43 , the condition for independent bubble velocities is satisfied under most flotation conditions.

The major difference in application of the Abrahamson model has been in the calculation of the particle and bubble fluctuating velocities. Levins \& Glastonbury (1972) were the first to derive an equation for particle motion in a turbulent fluid by assuming single frequency oscillations: 
$\frac{\overline{U_{p}^{\prime 2}}}{\overline{U_{f}^{\prime 2}}}=\frac{a \cdot T_{L}+b^{2}}{a \cdot T_{L}+1}$

where: $\quad a=\frac{36 \mu}{\left(2 \rho_{i}+\rho_{f}\right) \cdot d_{p}^{2}} \quad$ and $\quad b=\frac{3 \rho_{f}}{2 \rho_{i}+\rho_{f}}$

where $T_{L}$ is the Lagrangian time integral scale.

Abrahamson (1975) later built on this model by substituting the following equation for the Lagrangian time scale in the inertial sub-range of turbulence.

$T_{L}=0.7 \cdot \overline{U_{f}^{\prime 2}} / \varepsilon \quad$ (inertial sub-range only)

The Levins and Glastonbury equation could then be simplified by assuming it was applied to solid particles in a gas for which $b \cong 0$ and $a=1 / \tau_{p}$. This leads to the following equation:

$\overline{U_{p}^{\prime 2}}=\frac{\overline{U_{f}^{\prime 2}}}{1+1.5 \cdot \tau_{p} \cdot \varepsilon / \overline{U_{f}^{\prime 2}}} \quad$ (for $S t \square$ 1, inertial sub-range only)

Later, Liepe \& Mockel (1976) derived the following model for root mean squared fluctuating velocity from experimental work at intermediate Stokes numbers. This model is therefore thought to be more appropriate for flotation systems and has been commonly used together with the Abrahamson model in flotation literature (Schubert, 1999, Bloom \& Heindel, 2002, Pyke et al., 2003, Ralston et al., 2005).

$$
\sqrt{\overline{U_{i}^{\prime 2}}}=0.4 \cdot \frac{\varepsilon^{4 / 9} \cdot d_{i}^{7 / 9}}{v^{1 / 3}} \cdot\left(\frac{\rho_{i}-\rho_{f}}{\rho_{f}}\right)^{2 / 3} \quad \text { (intermediate } \mathrm{St} \text { ) }
$$


This model has recently been verified experimentally by Brady et. al. (2005). The results showed that the model closely predicts the fluctuating velocities of 80 micron glass beads under grid turbulence. Although the model was not derived for bubbles, it has been widely applied to bubbles in flotation literature (Schubert, 1999, Pyke et al., 2003, Ralston et al., 2005, Koh \& Schwarz, 2006). However, the results of Brady et. al. (2005) showed experimentally that the model greatly over predicted bubble fluctuating velocity for $1.2 \mathrm{~mm}$ bubbles.

An alternative equation for bubble fluctuating velocity is the model derived by Lee \& Erickson (1987):

$\overline{U_{b}^{\prime 2}}=C_{o} \cdot\left(\varepsilon \cdot d_{b}\right)^{2 / 3}$

where $\mathrm{C}_{\mathrm{o}}$ is given as 2 by Batchelor (1951). This model was however also shown to over predict the fluctuating velocity of $1.2 \mathrm{~mm}$ bubbles (Brady et al., 2005). Despite this, this model has been used in some modelling applications (Sherrel, 2004).

According to the results of Brady et. al. (2005), the Abrahamson model in fact gives the best approximation of bubble fluctuating velocity, due to the high bubble Stokes number. The Liepe and Mockel model provides the best approximation of particle fluctuating velocity for $80 \mu \mathrm{m}$ particles since it is derived for intermediate Stokes numbers. To date, these two models have not been used together in a simulation framework.

The major weakness of the above models is that they apply only to either the viscous or the inertial sub-range of turbulence. A more concise model should be able to account for collisions in both regimes as well as predict collision rates in the intermediate regimes. This problem has been considered by many researchers such as Williams \& Crane (1983), Yuu (1984) and most recently Kruis \& Kusters (1997). These models included an added mass term for particle motion in a liquid environment which is particularly relevant in flotation applications. The models have however only been derived for 
particle-particle interactions and are not yet applicable to heterogeneous collisions. The Kruis and Kusters model is similar in form to the Abrahamson model but differs in its calculation of fluctuating velocity, viz.

$\beta_{\text {shear }+ \text { accel }}=\sqrt{\frac{8 \pi}{3}} \cdot\left(r_{i}+r_{j}\right)^{2} \cdot \sqrt{w_{\text {shear }}^{2}+w_{\text {accel }}^{2}}$

$\frac{w_{\text {shear }}^{2}}{\mathrm{v}_{f}^{2}}=0.238 \cdot b\left(\frac{\mathrm{v}_{i}^{2}}{\mathrm{v}_{f}^{2}} \cdot \frac{\theta_{i}}{C_{c, i}}+\frac{\mathrm{v}_{j}^{2}}{\mathrm{v}_{f}^{2}} \cdot \frac{\theta_{j}}{C_{c, j}}+\sqrt{\frac{\theta_{i} \cdot \theta_{j}}{C_{c, i} \cdot C_{c, j}} \frac{\mathrm{v}_{i}^{2} \cdot \mathrm{v}_{j}^{2}}{\mathrm{v}_{f}^{2}}}\right)$

$\begin{aligned} & \frac{w_{\text {accel }}^{2}}{\mathrm{v}_{f}^{2}}=3 \cdot(1-b)^{2} \cdot \frac{\gamma}{\gamma-1} \cdot\left[\left(\theta_{i}+\theta_{j}\right)-\frac{4 \theta_{i} \cdot \theta_{j} \cdot \sqrt{\frac{1+\theta_{i}+\theta_{j}}{\left(1+\theta_{i}\right) \cdot\left(1+\theta_{j}\right)}}}{\left(\theta_{i}+\theta_{j}\right)}\right] \\ &\left(\frac{1}{\left(1+\theta_{i}\right)\left(1+\theta_{j}\right)}-\frac{1}{\left(1+\gamma \cdot \theta_{i}\right) \cdot\left(1+\gamma \cdot \theta_{j}\right)}\right)\end{aligned}$

where $\mathrm{w}_{\mathrm{i}}$ is the relative fluctuating velocity between the two particles, $\mathrm{b}$ is the added mass coefficient, $\gamma$ is the turbulence constant and $\theta_{\mathrm{i}}$ is the dimensionless particle relaxation time, given by the following equations:

$$
\begin{aligned}
& b=\frac{3 \rho_{f}}{2 \rho_{i}+\rho_{f}} \\
& \gamma=\frac{2 \cdot T_{L}^{2}}{(15 \cdot v / \varepsilon)^{4}} \\
& \theta_{i}=\frac{\tau_{i}}{T_{L}}
\end{aligned}
$$


The Kruis and Kusters model is able to predict a combined shear and accelerative mechanism of collision and gives predictions inbetween the Abrahamson and Saffman $\&$ Turner models as shown in the following figure:

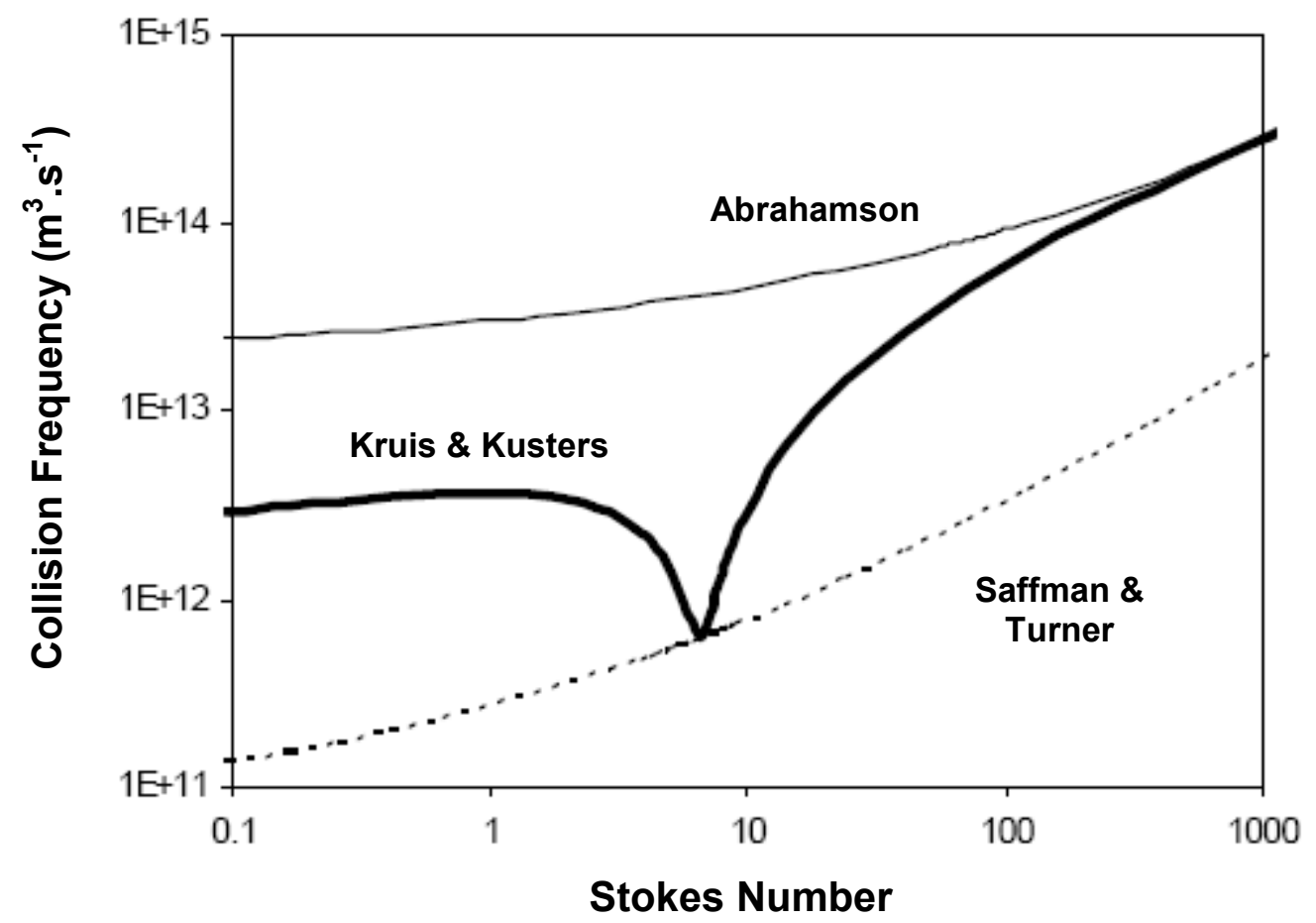

Figure 2.18 - Model Predictions for the Collision Frequency of Two Particles of Equal Density as a Function of Stokes Number with One Particle having a Constant Diameter of $100 \mu \mathrm{m}$ (from Sherrel, 2004).

Figure 2.18 shows that the collision rate predicted for the accelerative mechanism is approximately two orders of magnitude higher than that predicted for the shear mechanism. By incorporating added mass effects, Kruis \& Kusters obtained collision efficiencies higher than that of Saffman \& Turner, which reduced to the Abrahamson model at high particle Stokes numbers. The dip at an intermediate Stokes number of approximately 10 corresponds to the point where both particles have equal particle relaxation time, and collision is therefore entirely shear gradient driven. As the difference in Stokes number increases, the collision frequency increases due to increased relative motion of particles. 
The major weakness of the Kruis and Kusters model is that it is developed for particleparticle collision and only allows one particle density to be input into the added mass coefficient. The model therefore cannot be applied to flotation systems, given the vast difference in density between particles and bubbles. This therefore defines a task for the future development of flotation modelling.

Thus at present, only two major models exist for particle-bubble collision under turbulent conditions; the Saffman \& Turner model for low energy dissipation rates and low particle and bubble Stokes numbers, and the Abrahamson model for high energy dissipation rates and particle and bubble Stokes numbers. These have both been incorporated into CFD modelling (Koh \& Schwarz, 2006), with the constraint for their application being the condition of independent velocities (c.f. Equation 2.43). Theoretically it seems that neither model is truly applicable since particle Stokes numbers tend to be less than 1 for most flotation applications while bubble Stokes numbers are usually greater than 10 (c.f. Figures 2.7 and 2.8). Thus a combination model seems most appropriate for flotation applications, although no combination model has yet been developed for particle-bubble interactions. From the findings of this review, it would seem most appropriate to use the Abrahamson model for collision frequency since the Saffman \& Turner model is unable to account for the random motion of bubbles at high Stokes number. For most fine particles, St $<1$ and therefore the particle fluctuating velocity should be equal to the fluid fluctuating velocity. The bubble fluctuating velocity should be estimated from the Abrahamson model for $\mathrm{St}>$ 10, based on the experimental findings of Brady et. al. (2005). This would result in the following equation:

$$
Z_{p b}=\sqrt{8 \pi} \cdot\left(r_{p}+r_{b}\right) \cdot \sqrt{\overline{U_{f}^{\prime 2}}+\frac{\overline{U_{f}^{\prime 2}}}{1+1.5 \cdot \tau_{b} \cdot \varepsilon / \overline{U_{f}^{\prime 2}}}} \quad\left(\mathrm{St}_{\mathrm{p}}<1 ; \mathrm{St}_{\mathrm{b}}>10\right)
$$

However, this model still has the limitations that the assumption of a Guassian distribution of fluctuating velocity is only valid in the inertial sub-range of turbulence 
and only the bubble satisfies the condition of independent velocity under most practical conditions (Equation 2.43). Thus although much research is still required in developing models to describe particle-bubble interactions, the above model would seem to be most appropriate given the range of models which have been developed so far.

\subsubsection{Particle-Bubble Collision/Interception Models}

The turbulent collision frequency models in Section 2.3.1.2 account for the number of collision events occurring per unit time per unit volume. However these models do not include a description of the probability that a collision event will result in a successful interception of the particle with the bubble. Particles are small relative to bubbles and often follow the streamlines around bubbles rather than directly colliding with them. This problem has been extensively considered in quiescent systems, where numerous models have been derived based on laminar stream functions rather than the statistical description of turbulence. Studies in turbulent collision systems have subsequently accounted for interception effects by simply multiplying the turbulent collision rate $\left(\mathrm{Z}_{\mathrm{pb}}\right)$ by the probability of successful collision derived for a quiescent system $\left(\mathrm{P}_{\mathrm{c}}\right)$ to yield an overall rate of successful collisions (c.f. Equation 2.32). The following section provides a brief overview of some of the quiescent interception models developed.

Sutherland (1948) derived a collision probability model for quiescent systems based on the stream functions for potential flow. The model defined a critical approach radius within which the particle must approach the bubble in order to collide with it. The probability of collision was given by the ratio of critical approach cross sectional area to bubble cross sectional area and had the following form:

$$
P_{c, s u}=\frac{3 \cdot d_{p}}{d_{b}}
$$

This model assumes that particle inertia is negligible (i.e. St $<<1$ ), and that the bubble is a rigid sphere with streamlines defined by potential flow (i.e. $\operatorname{Re}_{b}>>1$ ). 
Later Gaudin (1957) derived a similar model based on the Stokes' regime of flow around the bubble surface $\left(\operatorname{Re}_{\mathrm{b}}<<1\right)$.

$$
P_{c, g}=\frac{3}{2} \cdot\left(\frac{d_{p}}{d_{b}}\right)^{2}
$$

This model is thought to be most appropriate for small bubbles less $100 \mu \mathrm{m}$ where the Stokes' regime of flow applies (Yoon, 2000). Later, Yoon \& Luttrell (1989) developed a model which incorporates a more generalized stream function for intermediate flow conditions.

$$
P_{c, Y-L}=\left(\frac{3}{2}+\frac{4 \cdot \mathrm{Re}^{0.72}}{15}\right) \cdot\left(\frac{d_{p}}{d_{b}}\right)^{2}
$$

When the Reynolds number is low, the expression reduces to Gaudin collision model for Stokes' flow and when the Reynolds number is high, the expression reduces to the Sutherland model for potential flow.

The major weakness of these collision models is that they do not account for particle inertia and inherently assume an immobile bubble surface. For this reason, Dai et. al. (1998) derived a more rigorous model for collision efficiency, based on the Sutherland collision model, which accounts for the mobility of the bubble surface and the effects of particle inertia, viz.

$P_{c, G S E}=P_{c, s u} \cdot \sin ^{2} \theta_{t} \cdot \exp \left[3 K_{3} \cdot \cos \theta_{t} \cdot\left(\ln \frac{3}{P_{c, s u}}-1.8\right)-\frac{9 K_{3}\left(\frac{2}{3}+\cos ^{3} \theta_{t}-\cos \theta_{t}\right)}{2 \cdot P_{c, s u} \cdot \sin ^{2} \theta_{t}}\right]$

where $P_{c, s u}$ is the Sutherland collision efficiency (c.f. Equation 2.56) and $\theta_{t}$ is the angle of tangency given by: 
$\theta_{t}=\arcsin \left[2 \cdot \beta \cdot\left(\sqrt{1+\beta^{2}}-\beta\right)\right]^{1 / 2}$

$\mathrm{K}_{3}$ and $\beta$ are dimensionless numbers, defined as:

$K_{3}=\frac{2 \cdot v_{b} \cdot\left(\rho_{p}-\rho_{f}\right) r_{p}^{2}}{9 \cdot \mu \cdot r_{b}}$

$\beta=\frac{2 \cdot P_{c, s u} \cdot f}{9 \cdot K_{3}}$

where $\mathrm{f}$ is a numerical factor characterising the hydrodynamic interaction between the particle and the bubble surface at short range.

This model has become known as the Generalised Sutherland Equation (GSE) and is thought to provide the best approximation of collision probabilities in flotation systems based on single bubble-particle experiments (Dai et. al., 2000).

\subsubsection{Particle-Bubble Attachment}

Particle-bubble attachment is the primary selective step in flotation. In order to successfully attach to a bubble, a particle must be sufficiently hydrophobic such that the time required to form a stable contact angle between the bubble and particle (induction time) is less than the time for which the particle and bubble are in contact (sliding time).

Based on this approach, Sutherland (1948) derived an equation for the sliding time based on the fluid stream function around a spherical bubble under potential flow conditions. This was then combined with the induction time $\left(t_{i}\right)$ to yield an expression for the probability of attachment, viz. 
$P_{a}=\sec h^{2}\left(\frac{3 \cdot v_{b p} \cdot t_{i}}{2 \cdot d_{b}}\right)$

where $\mathrm{v}_{\mathrm{bp}}$ is the relative velocity between the bubble and particle.

Recently, other researchers such as Yoon \& Mao (1996) and Sherrel \& Yoon (2005) have used an alternative modelling approach, based on the energy barrier between the bubble and particle, calculated using extended DLVO theory. Using this approach, the probability of attachment can be expressed in the following form:

$P_{a}=\exp \left(\frac{E_{B}}{E_{k}}\right)$

where $E_{B}$ denotes the energy barrier produced by surface forces such as van der Waals forces and electric double layer forces, which needs to be overcome for attachment to occur. $\mathrm{E}_{\mathrm{k}}$ denotes the energies available in the system to overcome this energy barrier.

\subsubsection{Particle-Bubble Detachment under Turbulence}

As shown in equation 2.32, particle-bubble detachment is usually accounted for as a probability function which describes the likelihood of the particle-bubble aggregate being ruptured by turbulent detachment forces directly after attachment. There is little consensus on the mechanism of detachment and the disruption of particle-bubble aggregates has been considered to occur as a result of a number of different detachment forces. These include centrifugal forces (Schulze, 1993), large-scale eddy fluctuations (Sherrel, 2004), hydrodynamic pressure fluctuations (Lu et. al., 1995) and collision of aggregates with eddies of similar size (Bloom \& Heindel, 2002). The following section will briefly review the structure of these different approaches.

Schulze (1993) approached the problem of modelling particle-bubble detachment using a force balance to determine a maximum flotation particle size. The net attachment and detachment forces were characterized by the Bond Number (Bo') which is the ratio of 
detaching to attaching forces. The probability of detachment was then assumed to be exponentially distributed based on experimental studies:

$P_{d}=\exp \left(1-\frac{1}{B o^{\prime}}\right) \quad$ and $\quad \mathrm{Bo}^{\prime}=\frac{F_{\text {detach }}}{F_{\text {attach }}}$

where the forces of attachment are the capillary and hydrostatic pressure forces. The forces of detachment are the apparent weight of the particle, the acceleration of the particle by the fluid and the capillary pressure of the gas bubble on the contact area of the particle. The calculation of these forces is made assuming spherical particles and is summarised by the following table:

Table 2.5: Attachment and Detachment Forces on the Particle-Bubble Aggregate

\begin{tabular}{|c|c|c|c|c|}
\hline \multirow{3}{*}{ 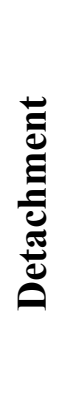 } & \multirow{3}{*}{ 递 } & Apparent Weight & $F_{w t}=\frac{4}{3} \cdot \pi \cdot R_{p}^{3} \cdot\left(\rho_{p}-\rho_{l}\right) \cdot g$ & $(2.66)$ \\
\hline & & Particle Acceleration & $F_{a c c}=\frac{4}{3} \cdot \pi \cdot R_{p}^{3} \cdot \rho_{p} \cdot a$ & $(2.67)$ \\
\hline & & $\begin{array}{c}\text { Capillary Pressure on } \\
\text { Particle }\end{array}$ & $F_{\sigma} \approx \pi \cdot R_{p}^{2} \cdot\left(\sin ^{2} \varphi\right) \cdot\left(\frac{2 \cdot \sigma}{R_{b}}-2 \cdot R_{b} \cdot \rho_{f} \cdot g\right.$ & $(2.68)$ \\
\hline \multirow{2}{*}{ 晜 } & \multirow{2}{*}{$\underbrace{0}_{0}$} & $\begin{array}{l}\text { Capillary Force on 3- } \\
\text { Phase Contact Line }\end{array}$ & $F_{c a}=-2 \pi \cdot R_{p} \cdot \sigma \cdot \sin \varphi \cdot \sin (\varphi+\theta)$ & $(2.69)$ \\
\hline & & Hydrostatic Pressure & $F_{h y d}=R_{p}^{2} \cdot\left(\sin ^{2} \varphi\right) \cdot \rho_{f} \cdot g \cdot z_{0}$ & $(2.70)$ \\
\hline
\end{tabular}

where:

$$
\begin{aligned}
& a=\text { Acceleration of particle by external fluid forces } \\
& \sigma=\text { surface tension } \\
& \varphi=\text { angle of capillary force } \\
& \theta=\text { contact angle } \\
& z_{0}=\text { height of liquid above the contact area }
\end{aligned}
$$


The particle acceleration term $(a)$ in equation 2.67 accounts for the effect of turbulence on the particle-bubble aggregate. The acceleration is approximated by the centrifugal acceleration of vortices of similar size to the particle-bubble aggregate. For the inertial sub-range of turbulence, this is given by:

$a \approx 1.9 \cdot \varepsilon^{2 / 3} /\left(R_{p}+R_{b}\right)^{1 / 3} \quad$ (inertial sub-range)

For aggregates in the viscous dissipation sub-range of turbulence, it is given by:

$a \approx 0.52 \cdot \varepsilon^{3 / 4} / v^{1 / 4} \quad$ (viscous dissipation sub-range)

Other researchers such as Sherrel \& Yoon (2005) have used an energy barrier approach together with the kinetic energy of detachment to calculate the detachment probability, viz.

$P_{d}=\exp \left(-\frac{W_{A}+E_{B}}{E_{k-D}}\right)$

where $\mathrm{W}_{\mathrm{A}}$ is the work of adhesion of the particle to the bubble, $\mathrm{E}_{\mathrm{B}}$ is the energy barrier between the disengaging particle and bubble, given by DLVO theory and $E_{k-D}$ is the kinetic energy of detachment. Interestingly, Sherrel \& Yoon (2005) considered the energy for detachment to arise not from the small scale eddies in the fluid, but rather from the large scale eddies, since these eddies contain the bulk of the turbulent kinetic energy. The kinetic energy of detachment is therefore given by the fluctuating velocity of these eddies, viz.

$E_{k-D}=\frac{1}{2} \cdot\left(m_{1}+m_{2}\right) \cdot \overline{U_{\text {large }}^{\prime 2}}$

For a stirred tank environment, this velocity was estimated using the tip speed of the impeller squared: 
$\overline{U_{\text {large }}^{\prime 2}}=\left(R_{\text {Imp }} \cdot \omega\right)^{2}$

Lu et. al. (1995) used a similar approach, defining an energy barrier which must be overcome for detachment to occur. However the dominant disruption action of turbulent flow was considered to be the hydrodynamic pressure fluctuations under turbulence. Following this methodology, the mean disruptive stress experienced by an aggregate is given by:

$$
\sqrt{\overline{\Delta P^{\prime 2}}}=1.0 \cdot \overline{U_{f}^{\prime 2}} \approx 2 \cdot \rho \cdot\left(\varepsilon \cdot d_{a}\right)^{2 / 3}
$$

The strength of the aggregate based on the inertial sub-range was then estimated using the expression developed by Muhle \& Domasch (1991). These were combined into a critical Weber number, analogous to bubble break-up where for $\mathrm{We}>1$, pressure fluctuations were sufficient to disrupt aggregates. The Weber number is given by:

$$
W e=\frac{2 \cdot \rho_{a} \cdot \varepsilon^{11 / 12} \cdot d_{a}^{5 / 3} \cdot d_{p}^{2}}{F_{A} \cdot v^{3 / 4}}
$$

where $F_{A}$ is the force of adhesion, calculated from the energy barrier between the bubble and particle. Based on their calculations, the Weber number for quartz particles with an amine collector in the impeller zone was found to exceed 1 for particles from 40-76 micron at all $\mathrm{pH}$ levels. The Weber number also exceeded 1 for 10-20 micron particles provided that the $\mathrm{pH}$ was greater than 10 .

Bloom \& Heindel (2002) were the first to consider particle-bubble detachment as a detachment frequency rather than a probability of detachment. Their proposed model was adapted from similar work in particle aggregation by Mika \& Fuerstenau (1968), where the disruption of flocs was thought to be caused by eddies of comparable size to 
the flocculation aggregates. Using this approach, they modelled contacting as the sum of a forward and reverse reaction, viz.

$$
\frac{d N_{p}}{d t}=-Z_{p b} \cdot N_{b} \cdot N_{p} \cdot P_{c} \cdot P_{a}+Z_{p b}^{\prime} \cdot N_{p b} \cdot P_{d}
$$

where $Z^{\prime}{ }_{p b}$ is the volumetric rate of detachment in the system and $\mathrm{N}_{\mathrm{pb}}$ is the number of attached bubbles per unit volume. While this equation is novel in its form, the application of it is difficult since it requires an estimation of the number of attached bubbles in the system $\left(\mathrm{N}_{\mathrm{pb}}\right)$ and therefore requires a complex population balance-type modelling approach to account for the state of individual bubbles in the system. The detachment frequency is given by the following equation:

$$
Z_{p b}^{\prime}=\frac{\sqrt{C_{1}} \cdot \varepsilon^{1 / 3}}{\left(d_{p}+d_{b}\right)^{2 / 3}} \quad \text { (inertial sub-range only) }
$$

where $C_{1}$ is an empirical value between 1.61 and 2.33 . This particular model gives a much weaker dependence of detachment on energy dissipation compared with other models which indicate that detachment follows energy to the power of between $2 / 3$ and $11 / 12$.

\subsubsection{Rheological Characteristics of Mineral Suspensions}

The previous section has shown that the particle-bubble contacting rate in flotation cells is highly dependent on the fluid micro-turbulent environment, as characterised by the local energy dissipation rate and the viscosity of the fluid. Important to note however is that the current range of fundamental flotation models are derived for Newtonian fluid conditions, on which the statistical description of turbulence is based. While this may be a reasonable assumption at low solids fractions, the rheology of mineral suspensions in most industrial applications tends to exhibit non-Newtonian behaviour. The following section presents a brief overview of the modelling of slurry rheology in industrial mineral slurries as well as some of the equipment used to measure rheological 
characteristics. This overview is derived from Johnson et. al. (2000), He et. al. (2004) and Burdukova (2007).

\subsubsection{Rheology Fundamentals}

Rheology is study of the deformation and flow of fluid and suspensions. A fluid's rheological characteristics define the fluid's resistance to deformation when a shear stress force is applied. For Newtonian fluids, the relationship between the applied shear stress and shear rate is linear and is characterised by the viscosity of the fluid, given by:

$\mu=\frac{\tau_{s}}{\dot{\gamma}}$

where $\tau_{\mathrm{s}}$ is the shear stress in $\mathrm{Pa}$ and $\gamma$ is the shear rate in the fluid in $\mathrm{s}^{-1}$.

For many fluids however, the relationship between shear stress and shear rate is highly non-linear and is typically characterised by a graph of shear stress vs. shear rate, known as a rheogram. Some examples of the rheograms of different fluid types are shown in Figure 2.19.

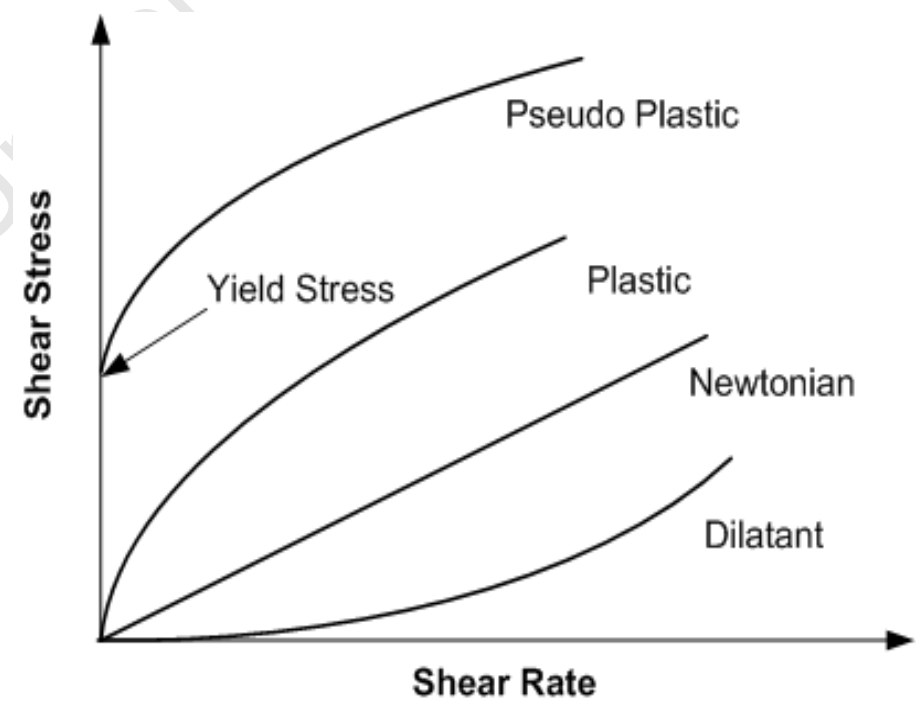

Figure 2.19 - Shear Stress vs. Shear Rate Curves for Different Fluids (Burdukova, 2007) 
Most industrial mineral slurries exhibit pseudo plastic rheological characteristics and are characterised by a non-zero y-intercept on the rheogram, known as a yield stress. This yield stress is defined as the minimum shear stress which must be applied to the fluid in order for deformation to take place and is a characteristic property of pseudoplastic fluids.

The magnitude of the slurry viscosity and yield stress varies widely between different slurries and is largely determined by the strength of the inter-particle surface forces in the suspension. As the inter-particle forces increase, for example as the zeta potential of the particles approaches zero, particles tend to aggregate, thereby increasing the slurry's resistance to deformation and increasing the slurry yield stress and viscosity. Slurry yield stress and viscosity are also known to increase with increasing percent solids due to the increased number of inter-particle interactions. Yield stress and viscosity also decrease with decreasing particle size due to the larger overall particle surface area available for interaction (He et al., 2004). Particle shape also plays an important role in determining rheological characteristics, since more fibrous or platy minerals exhibit a greater surface area per unit volume than other minerals. These minerals are therefore more prone to interacting with one another in the mineral suspension, thereby increasing the slurry yield stress and viscosity.

The yield stress of a mineral suspension is a particularly difficult property to measure since it requires an infinitesimal shear rate be applied to the fluid. This value is therefore usually inferred from rheological curves using rheological models. The simplest and most widely used of these models is the Bingham model, which is given by the following equation:

$\tau_{s}=\mu_{B} \cdot \dot{\gamma}+\tau_{B}$

where $\mu_{\mathrm{B}}$ is the Bingham viscosity, or gradient of the shear stress - shear rate curve, and $\tau_{\mathrm{B}}$ is the Bingham yield stress, or y-intercept. This model is useful for characterising pseudo-plastic fluids, particularly at high shear rates $\left(\gamma>50^{-1}\right)$, where the relationship 
between shear stress and shear rate closely approximates linearity. The Bingham model does however tend to overestimate the yield stress of the slurry, although it still provides a useful relative measure of yield stress when comparing different slurry types.

At lower shear rates $\left(\gamma<50^{-1}\right)$, the shear stress - shear rate relationship of pseuso-plastic fluids starts to deviate from linearity, leading other researchers to develop models which more closely approximate this behaviour. One such example is the Casson model which makes use of a square-root relationship to more accurately represent the shear stressshear rate relationship at low shear rate:

$\sqrt{\tau_{s}}=\sqrt{\tau_{C}}+\sqrt{\mu_{C} \cdot \dot{\gamma}}$

While this model does give a more accurate estimate of the true slurry yield stress, it is most commonly used at low shear rates where the Bingham model tends to over predict the shear stress. At higher shear rates however, the Bingham model generally provides an adequate representation of the shear stress - shear rate relationship.

Another common approach to the modelling of non-Newtonian fluids, is to define an apparent viscosity of the fluid. This apparent viscosity characterises the ratio of the shear stress to shear rate at a given point on the rheogram and is therefore a strong function of the shear rate applied to the fluid. Figure 2.20 shows the typical relationship obtained between the apparent viscosity of a pseudo plastic fluid and the shear rate in the fluid. This is compared with a Newtonian fluid to emphasise the non-linearity of the apparent viscosity in a pseudo-plastic fluid. 


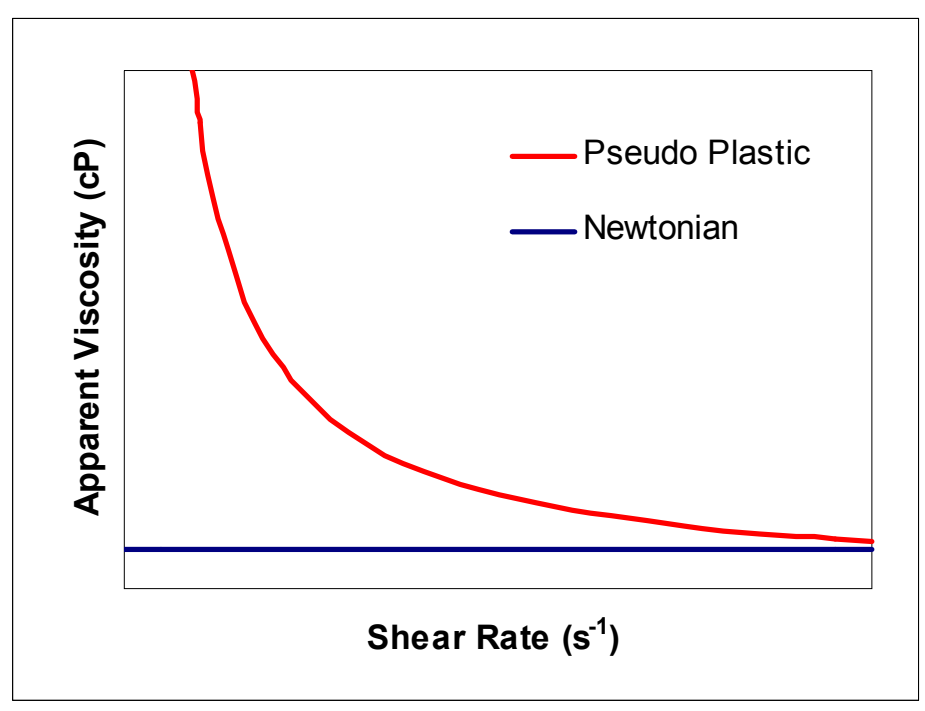

Figure 2.20 - Apparent Viscosity of a Typical Pseudo Plastic Fluid compared with a Newtonian Fluid

Figure 2.19 illustrates that for a typical pseudo-plastic fluid the ratio of shear stress to shear rate (the apparent viscosity) increases asymptotically as shear rate decreases. The apparent viscosity tends to infinity as the shear rate approaches zero. It can therefore be seen that the effect of the slurry yield stress is most apparent on the rheological behaviour of the fluid at low values of shear rate.

As was detailed in Section 2.3.1, the current range of fundamental models describing the relationship between rheology and particle-bubble contacting are derived for Newtonian systems and the description of particle-bubble contacting under nonNewtonian conditions has yet to be derived. However, it is speculated that the apparent viscosity may be used in place of the Newtonian viscosity when applying the fundamental models, as this quantity adequately defines the relationship between shear stress and shear rate at a given point in the fluid. The drawback of this approach is that it requires a detailed knowledge of the shear rate distribution in the cell in order to map the apparent viscosity distribution in the cell. This information is generally not readily available and relies on computationally intensive techniques such as CFD modelling. 


\subsubsection{Measurement of Rheology}

The rheology of mineral slurry is generally measured using rotational rheometers. These devices consist of a cup and spindle arrangement where a known shear rate is applied to the fluid by rotating the spindle and the resulting shear stress is measured using a torque sensor fitted to the shaft of the spindle. The geometry of these devices varies widely according to the required sensitivity of the measurement, but typical rheometers consist of either a single or double gap arrangement.

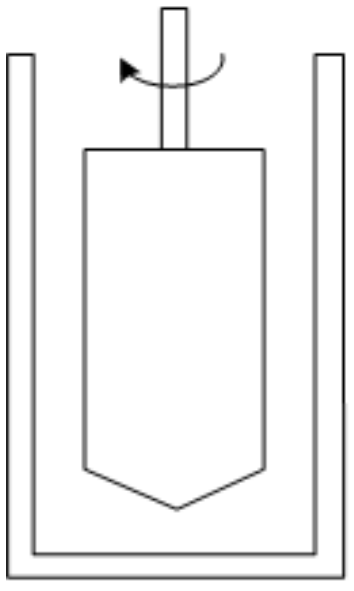

Single Gap

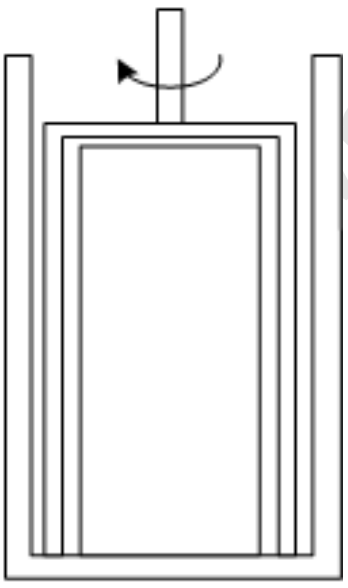

Double Gap

Figure 2.21 - Schematic of a Typical Cup and Spindle Rheometer (Burdukova, 2007)

Single gap rheometers are usually sufficient for measurement of highly viscous material where the shear stress at a given shear rate is large and therefore the torque transmitted to the shaft of the spindle is sufficient to be accurately measured. For less viscous material however, a double gap rheometer may be required. This arrangement allows a a greater torque to be transmitted to the shaft of the spindle by providing substantially more surface area in contact with the slurry. This increased torque can then be measured to a greater level of accuracy.

Several other on-line devices have been devised over the years in order to provide an on-line measurement of slurry viscosity. These devices generally consist of a spindle which is inserted into a slurry pipe for on-line measurement of shear stress. The 
drawback of these devices is that they are only able to measure the shear stress at a single shear rate point and therefore only obtain the apparent viscosity at a given shear rate, rather than the entire rheogram. These devices do however provide a useful relative measure of slurry viscosity which may be used for control purposes in industrial processes.

Rheology is an increasing important area of flotation research, particularly as minerals processing operations start to employ ultra-fine grinding techniques in order liberate valuable minerals. As the fineness of grind increases, so the slurry yield stress and viscosity increase, with detrimental effects on flotation cell hydrodynamics (Arratia et al., 2006, Deglon et al., 2007, Nel et al., 2007). Furthermore, as was shown in section 2.3.1, increased slurry viscosity inhibits particle-bubble contacting rates by damping the turbulence in the fluid. This is anticipated to decrease the flotation kinetics and necessitate the use of more sophisticated flotation techniques.

\subsubsection{Experimental studies}

Until now, experimental investigations into the effect of hydrodynamics on particlebubble contacting have been limited to conventional impeller-driven flotation cells. In most cases a bench scale Rushton turbine driven cell of $+/-2.25$ litres has been used. A summary of these studies is given in Table 2.3. While much useful information has been gained, the studies are still limited by the significant difference in energy dissipation rate between the impeller zone and the bulk fluid of an impeller-driven cell. This means that the studies are limited in their ability to attribute the observed flotation kinetics to turbulence in the impeller zone or turbulence in the bulk fluid, making extrapolation of the results to larger cells extremely difficult. 
Table 2.6: Summary of Previous Experimental Studies on Hydrodynamics and Flotation Rate at Constant Bubble Size

\begin{tabular}{|l|l|l|l|l|}
\hline \multicolumn{1}{|c|}{ Author } & \multicolumn{1}{|c|}{ Energy Range } & \multicolumn{1}{|c|}{ Ore } & Hydrophobicity & Particle Size \\
\hline $\begin{array}{l}\text { Ahmed \& } \\
\text { Jameson (1985) }\end{array}$ & $\begin{array}{l}0.01 \text { to } 2.5 \\
\mathrm{~W} / \mathrm{kg}^{*}\end{array}$ & $\begin{array}{l}\text { Latex, } \\
\text { Quartz, } \\
\text { Zircon }\end{array}$ & $\begin{array}{l}\text { Moderate } \\
\text { collector dosage }\end{array}$ & $<42$ micron \\
\hline Deglon (1998) & $\begin{array}{l}0.1 \text { to } 1.9 \\
\mathrm{~W} / \mathrm{kg}\end{array}$ & Quartz & $\begin{array}{l}\text { Low collector } \\
\text { dosage }\end{array}$ & $<32$ micron; \\
& $\begin{array}{l}0.45 \text { to } 1.8 \\
\mathrm{~W} / \mathrm{kg}\end{array}$ & $\begin{array}{l}\text { Quartz } \\
\text { Chalcopyrite, } \\
\text { Galena }\end{array}$ & $\begin{array}{l}\text { Advancing } \\
\text { contact angle of } \\
53^{\circ} \text { to } 80^{\circ}\end{array}$ & $<75$ micron \\
\hline Pyke (2004) & $\begin{array}{l}1.25 \text { to } 12.6 \\
\mathrm{~W} / \mathrm{kg} ;(0.15 \text { to } \\
1.8 \mathrm{~W} / \mathrm{kg})^{*}\end{array}$ & $\begin{array}{l}\text { Quartz } \\
\text { Advancing } \\
\text { contact angle of } \\
80^{\circ}\end{array}$ & $<100$ micron \\
\hline Newell (2006) & & \\
\hline
\end{tabular}

Ahmed \& Jameson (1985) were the first to investigate the effect of hydrodynamics on particle-bubble contacting. They used a batch impeller-driven stirred tank in which bubbles could be generated independent of the turbulence in the cell. In their investigation they considered bubble size, particle size, particle density as well as impeller speeds. Their results showed an up to 50 fold increase in rate constant when bubble size was decreased from $665 \mu \mathrm{m}$ to $75 \mu \mathrm{m}$. Interestingly, in the case of the denser particles (quartz \& zircon), the benefit of this smaller bubble size was diminished as the impeller speed was increased due to increased detachment. It was therefore concluded that for these dense particles, optimal flotation conditions were at the minimum bubble size and lowest impeller speed for solid suspension.

* Back-calculated using a power number of 5.5 for a six-bladed Rushton turbine (Rushton et. al., 1950). 
In general, it was found that rate constant increased with particle size. However, for the denser particles (zircon), the rate constant went through a maximum with particle size due to detachment of coarser material. At a constant bubble size of $665 \mu \mathrm{m}$, an increase in impeller speed led to an increase in rate constant. However, for the finer bubble sizes where detachment forces are stronger, increases in impeller speed produced only marginal increases in rate constant and in some cases, decreased the rate constant. Thus it was shown that the optimal recovery point was a function of not only particle size but bubble size as well.

Deglon (1998) investigated the flotation response of quartz as a function of hydrodynamic factors such as power dissipation and bubble size for a 2.25 litre batch flotation cell. At constant bubble size and using a Rushton turbine, an increase in flotation kinetics was obtained when increasing power input from $0.1 \mathrm{~W} / \mathrm{kg}$ to 0.65 $\mathrm{W} / \mathrm{kg}$, for both sub 32 micron and sub 100 micron quartz particles. As power input was further increased, the flotation rate decreased sharply in both size fractions, due to increased particle-bubble detachment. This resulted in an optimum flotation power input which was noted to be lower than that required for effective bubble break-up. It was therefore suggested that the energy conditions required for optimal particle-bubble contacting were in fact significantly lower than that required to produce small bubbles. The study was however conducted at low particle hydrophobicities which may partly account for the significant detachment rates observed.

Pyke (2004) investigated the effect of agitation and hydrophobicity on the flotation of quartz, chalcopyrite and galena particles. He found that when the contact angle of quartz was increased from $73^{\circ}$ to $80^{\circ}$ (advancing contact angle) at $0.45 \mathrm{~W} / \mathrm{kg}$, the overall rate of flotation increased dramatically for all particle sizes, particularly for the coarser particle sizes as the rate of detachment decreased significantly. When increasing the power input from $0.45 \mathrm{~W} / \mathrm{kg}$ to $1.45 \mathrm{~W} / \mathrm{kg}$, the flotation rate of quartz particles with an advancing contact angle of the $73^{\circ}$ decreased due to increased detachment for all particle size fractions. This indicates that for particles of this hydrophobicity, the system was largely detachment dominated. When the hydrophobicity was increased to $80^{\circ}$, the flotation rates of the fine particles remained constant and only the rate of particles 
greater than 30 micron decreased as power input was increased from 0.45 to $1.80 \mathrm{~W} / \mathrm{kg}$, due to detachment. This shows that hydrophobicity has a large effect on the detachment rate of particles, particularly for coarse particles.

In contrast, the flotation of chalcopyrite particles was found to be improved by increased power input for all particle size ranges despite the lower range of contact angles measured $\left(56^{\circ}\right.$ and $\left.76^{\circ}\right)$. This indicates that there must be an additional effect of ore type on the flotation rate over and above the hydrophobicity of the particles.

Newell (2006) investigated the effect of hydrodynamics on quartz flotation in three different size flotation cells driven by Rushton turbines. The investigation centred on energy inputs between 1.25 and $12.6 \mathrm{~W} / \mathrm{kg}$. He found that for quartz with a contact angle of $80^{\circ}$, the rate constant increased steadily with energy up to $4.1 \mathrm{~W} / \mathrm{kg}$ before remaining constant until $12.6 \mathrm{~W} / \mathrm{kg}$. One would therefore expect to obtain optimal flotation performance somewhere between 4.1 and $12.6 \mathrm{~W} / \mathrm{kg}$. This value would be an order of magnitude higher than the value obtained by Deglon (1998) and Pyke (2004). The reason for this discrepancy is largely unclear, however calculation shows that there is a large discrepancy between the power numbers obtained in each system.

In general, all studies have shown an increase in the flotation rate with increasing particle size, except at high power inputs and gas rates, where the rate constants of the coarser particles ( +60 micron) where found to plateau or decrease slightly, due to increased detachment.

Overall, the studies of Ahmed \& Jameson (1985), Deglon (1998) and Pyke (2004) seem to indicate that the point at which detachment starts to dominate in the system may in fact be lower than that required to produce small bubbles. This would indicate that the high energy dissipation rates obtained at the impeller are highly effective in producing small bubbles but result in substantial particle-bubble detachment. This seems to suggest that optimal conditions for particle-bubble contacting may in fact be found in a region slightly further away from the impeller where dissipation rates are somewhat lower. This was also shown in the results of Deglon (1998) who found that in the 
absence of gas dispersion effects, a Rushton turbine impeller provided improved flotation performance compared to a spinning disc, since the energy dissipation in the fluid was distributed over a larger volume of the cell. This hypothesis is supported by the findings of recent CFD simulations which show a negative attachment rate close to the impeller due to large detachment rates in the impeller zone (Koh \& Schwarz, 2007).

\subsubsection{Summary of the Effect of Hydrodynamics on Particle-Bubble Contacting}

The preceding section has reviewed the current understanding of the effect of hydrodynamic factors such as energy dissipation rate and viscosity on the particlebubble contacting rate in turbulent flotation systems. It can be seen that the hydrodynamics of the fluid affect both the rate of collision and the rate of detachment of particles from bubbles. The rate of collision is thought to increase with increasing energy dissipation rate to the power of 0.5 for a shear mechanism of collision (c.f. Equation 2.41), and to the power of 0.44 for an accelerative mechanism of collision (c.f. Equation $2.42 \& 2.47)$. However, as the energy dissipation rate in the system is further increased, this increased rate of collision is counterbalanced by an increase in the probability of detachment of particles from bubbles. This probability of detachment is often characterised by an exponential decay function which starts to dominate the kinetics at higher energy dissipation rates, resulting in a decrease in the overall flotation rate with increasing power input. This results in an optimum flotation power input which increases with decreasing particle size and increasing particle hydrophobicity.

In terms of viscosity, the statistical description of turbulence predicts that an increase in the fluid viscosity results in a damping of the micro-turbulence in the fluid. This is characterised by an increase in the size of the viscous dissipation eddies and a decrease in the mean shear rate contained within these eddies. This, in turn, leads to a decrease in the particle-bubble contacting rate with increasing viscosity to the power of 0.5 for a shear mechanism (c.f. Equation 2.41), and to the power of approximately 0.33 for an accelerative mechanism (c.f. Equation $2.42 \& 2.47$ ). 
The description of particle-bubble contacting under non-Newtonian conditions has yet to be derived. However, it is speculated that the apparent viscosity may be used in place of the Newtonian viscosity when applying the fundamental models as this quantity adequately defines the relationship between shear stress and shear rate at a given point in the fluid. The drawback of this approach is that it requires a detailed knowledge of the shear rate distribution in the cell in order to map the apparent viscosity distribution in the cell.

The particle-bubble contacting rate is also a strong function of the bubble size, which affects all sub-processes including the collision rate, the probability of collision and the probability of detachment. An investigation into the effect of hydrodynamics on particle-bubble contacting is therefore best performed at a constant bubble size so as to distinguish the direct effect of hydrodynamics on the contacting rate from the indirect effect of the bubble size of the flotation rate. For this reason, most experimental studies on energy and particle-bubble contacting have been performed using specialised frits for bubble generation. These frits produce a fine bubble size which effectively mitigates further bubble break-up by the impeller. This means that trends in flotation rate can be attributed to changes in hydrodynamics rather than changes in the bubble size characteristics.

The particle-bubble contacting rate is also thought to be proportional to the number of bubbles per unit volume in the cell (c.f. Equation 2.32). This number density of bubbles can be calculated using the following equation (Pyke et al., 2003).

$$
N_{b}=\frac{6 \cdot G_{f r}}{\pi \cdot d_{b}^{3} \cdot V_{c e l l}} \cdot \tau_{g}
$$

where $G_{f r}$ is the gas flow rate, $V_{\text {cell }}$ is the volume of the cell and $\tau_{\mathrm{g}}$ is the gas residence time in the cell, given by (Deglon et. al., 1999): 


$$
\tau_{g}=\frac{\phi \cdot V_{\text {cell }}}{J_{g} \cdot A}
$$

where $\phi$ is the gas hold-up, $\mathrm{J}_{\mathrm{g}}$ is the superficial gas velocity and $\mathrm{A}$ is the cross sectional area of the cell.

By combining equations 2.32, 2.80 and 2.81, we see that the framework used by fundamental flotation models (c.f. Equation 2.32) predicts that the flotation rate $(\mathrm{k})$ is direct proportional to the gas hold-up $(\phi)$. This relationship arises from the volumetric basis on which the model framework is derived. It is however very difficult to verify this relationship experimentally in a conventional system, since the gas hold-up is intimately related to the bubble size in the flotation cell.

An increasing body of literature has shown that in fact a strong relationship exists between the flotation rate constant and the bubble surface area flux in the cell (Gorain et. al., 1997, Deglon et al., 1999, Heiskanen, 2000). This body of evidence has led many researchers to develop models for flotation on a flux basis rather than a volumetric basis (Franzidis \& Manlapig, 1999, Deglon, 2002, Harris et. al., 2002). In systems such as a conventional cell, these two approaches appear to be largely equivalent since an increase in the bubble surface area flux is generally associated with an increase in the gas hold-up and vice versa.

In an OBC however, studies on single bubble trajectories have shown that the residence time of bubbles in the cell is not only affected by the bubble surface area flux, but also by the reversing flow which is generated under oscillatory flow conditions (Oliveira et. al., 2003). The OBC may therefore provide a useful means of varying the gas hold-up in the system independently of the bubble size produced. Such an investigation is considerably more difficult in a conventional system and the OBC may therefore be able to provide additional insights into the validity of these modelling approaches. 


\subsubsection{The Efficacy of the OBC as a Research Tool}

Section 2.3 has presented a review of the effect of hydrodynamics on the rate of particle-bubble contacting in flotation. It can be seen that the range of fundamental models in the literature are largely independent of the mode of power input to the system and are dependent only on the energy dissipation rate produced as a result of that power input and the viscosity of the fluid. Until now, the effect of power dissipation on particle-bubble contacting has only been investigated in stirred systems where the disparity of power dissipation in fluid limits one's ability to attribute trends to changes in the impeller zone or changes in the bulk zone of the cell.

Given the OBC's unique mode of agitation, it is believed that a distinct opportunity exists for the investigation of the effect of hydrodynamics on particle-bubble contacting in a markedly different environment. This provides an opportunity to evaluate the relative importance of the mode of agitation in determining the particle-bubble contacting rate versus the importance of the micro-turbulent environment produced as a result of that agitation. A unique attribute of the $\mathrm{OBC}$ is that the flow patterns in the fluid are thought to be independent of the slurry rheology for $\operatorname{Re}_{\mathrm{o}}>1000$. As shown in section 2.3.1, rheology has a significant impact on the micro-turbulent environment in the fluid. It is believed that this independence of the flow patterns on slurry rheology provides a unique opportunity to vary the micro-turbulent environment independently of the large scale eddies and mixing in the fluid. It is believed that an investigation of this kind will therefore enhance our understanding of the role which micro-turbulence plays in promoting particle-bubble contacting.

In addition, the OBC's more even distribution of shear allows the extraction of trends in flotation rate with power dissipation, without the added complication of the disparity of power dissipation which occurs in stirred systems. This attribute is believed to make the OBC a more appropriate environment in which to evaluate the effect of power dissipation on particle bubble contacting. 
It should be noted however that the flow in an OBC is highly anisotropic and inherently unsteady with time. The oscillatory motion of the fluid has also been shown to produce complex particle-bubble trapping effects which are not present in conventional systems and introduce added complications of their own. This oscillatory nature of the flow may give rise to alternative mechanisms of contacting not yet proposed in the literature, meaning that the direct application of fundamental flotation models to this system may not be valid. The isolation of such an alternative mechanism would however highlight some of the shortfalls of the current fundamental models and thereby inform their future development.

\subsection{Literature Summary}

This chapter has provided an overview of the existing body of knowledge in three key areas which are relevant to this study. These are the development of novel flotation cells, the characteristics of Oscillatory Baffled Columns and the modelling and investigation of the effect of hydrodynamics on particle-bubble contacting.

It was shown that the two most widely used flotation cell technologies, the mechanical cell and the column cell, are under threat from the increasing prevalence of fine mineral slurries, since fine particle recovery remains an issue in these flotation systems. Due to this challenge, a number of novel cells have been developed over the years. It was shown that one such promising design for improving fine particle recovery was the agitated column cell, which had the ability to optimise the hydrodynamic environment for effective particle-bubble contacting of fine particles. Despite the evidence of improvements in flotation rate, this cell has yet to be tested industrially.

The review then detailed the development and characteristics of Oscillatory Baffled Columns, which are similar in design to the agitated column, but make use of an alternative agitation mechanism. These cells also possess a number of attributes which have made them highly effective in other industrial applications. It was hypothesised that the even distribution of shear rate in the fluid, together with the variability of power input, would make this type of cell highly effective as a novel flotation device for fine 
particle recovery. Furthermore, the device was shown to be able to produce bulk flow patterns which are independent of fluid rheology for $\operatorname{Re}_{0}>1000$. It was hypothesised that this attribute would make the OBC effective in the flotation of slurries exhibiting complex rheology.

It was also noted that the OBC possesses several attributes which would make it an effective research tool for evaluating the effect of power input on particle-bubble contacting. The current understanding of the effect of hydrodynamic parameters such as energy dissipation rate and viscosity on particle-bubble contacting was then reviewed, both in terms of the fundamental models which have been developed, and in terms of the experimental studies which have been conducted. It was shown that the particlebubble contacting rate is thought to be controlled by the micro-turbulent environment of the fluid, which is characterised by the inertial and viscous dissipation sub-range eddies. The characteristics of these eddies are determined by the distribution of the energy dissipation rate and the apparent viscosity in the fluid and are therefore thought to be independent of the mode of power input to the fluid.

Given the OBC's unique agitation mechanism, it was hypothesised that this cell presents a distinct opportunity to investigate the effect of the hydrodynamic environment on the particle-bubble contacting rate in a system employing an alternative mode of power input. It is anticipated that such an investigation would highlight the relative importance of the mode of agitation in determining the particle-bubble contacting rate and thereby inform the further development of fundamental flotation models.

Based on the findings of this review, the literature seems to support the hypothesis that the $\mathrm{OBC}$ will be effective as a novel cell for fine particle recovery, given its similar design to the agitated column together with its flexibility of power input and even distribution of shear rate. Furthermore, the literature suggests that the cell may also be effective in the treatment of rheologically complex ores, due to the independence of bulk flow patterns on slurry rheology. The hypothesis that the OBC can be used as a research tool for evaluating the effect of power input on particle-bubble contacting also 
seems to have merit, since the OBC's alternative mechanism of agitation may provide new insights in the effect of the hydrodynamic environment on particle-bubble contacting which could not be obtained in conventional stirred systems.

In order to test these hypotheses, it seems appropriate to first evaluate the OBC's flotation performance using quartz, in order to provide a basis for comparison of this study with previous studies in stirred systems (c.f. Table 2.6). Following the methodology of these studies, this investigation should ideally be conducted at constant bubble size so as to clarify the direct effect of power dissipation on particle-bubble contacting, independent of bubble size effects.

The cell has also been reported to provide good mixing conditions under low oscillatory Reynolds number conditions which may affect the overall recoveries obtained. Furthermore, the gas hold-up in the cell is also likely to be affected by the increased reversing flow which is obtained in an $\mathrm{OBC}$, and may have an effect of the flotation kinetics. The investigation should therefore be coupled with an investigation of the effect of cell operating conditions on characteristics such as mixing, bubble size and gas hold-up in order to inform the interpretation of flotation results.

Given the hypothesised effectiveness of the $\mathrm{OBC}$ in treating rheologically complex mineral slurries, the study should then be extended to evaluating the OBC's performance in the flotation of a fine, rheologically complex nickel ore slimes, which is difficult to treat using conventional cell technology. This test work would also provide an opportunity to vary the micro-turbulent environment independently of the large scale eddies and flow patterns in the cell. This offers an opportunity to assess the relative importance of micro-turbulence in determining the particle-bubble contacting rate, and assess the degree to which the flotation response is affected by changes in slurry rheology. 


\section{Chapter 3}

\section{Materials and Methods}

The following chapter details the materials and methods used in the characterisation and flotation testing of the Oscillatory Baffled Column. The design of the OBC is first described before the test procedures and methods of calculation are outlined.

\subsection{The Oscillatory Baffled Column}

The Oscillatory Baffled Column consists of a standard PVC column section, fitted with a moving baffle-cage which can be operated at a variety of frequencies and amplitudes. The baffle cage is made up of 6 stainless steel orifice plates which are oscillated by a piston and cam located above the cell. The piston is driven by a $0.37 \mathrm{~kW}$ variable speed motor and has an adjustable yoke to vary the amplitude of oscillation. A schematic of the cell is given in Figure 3.1: 


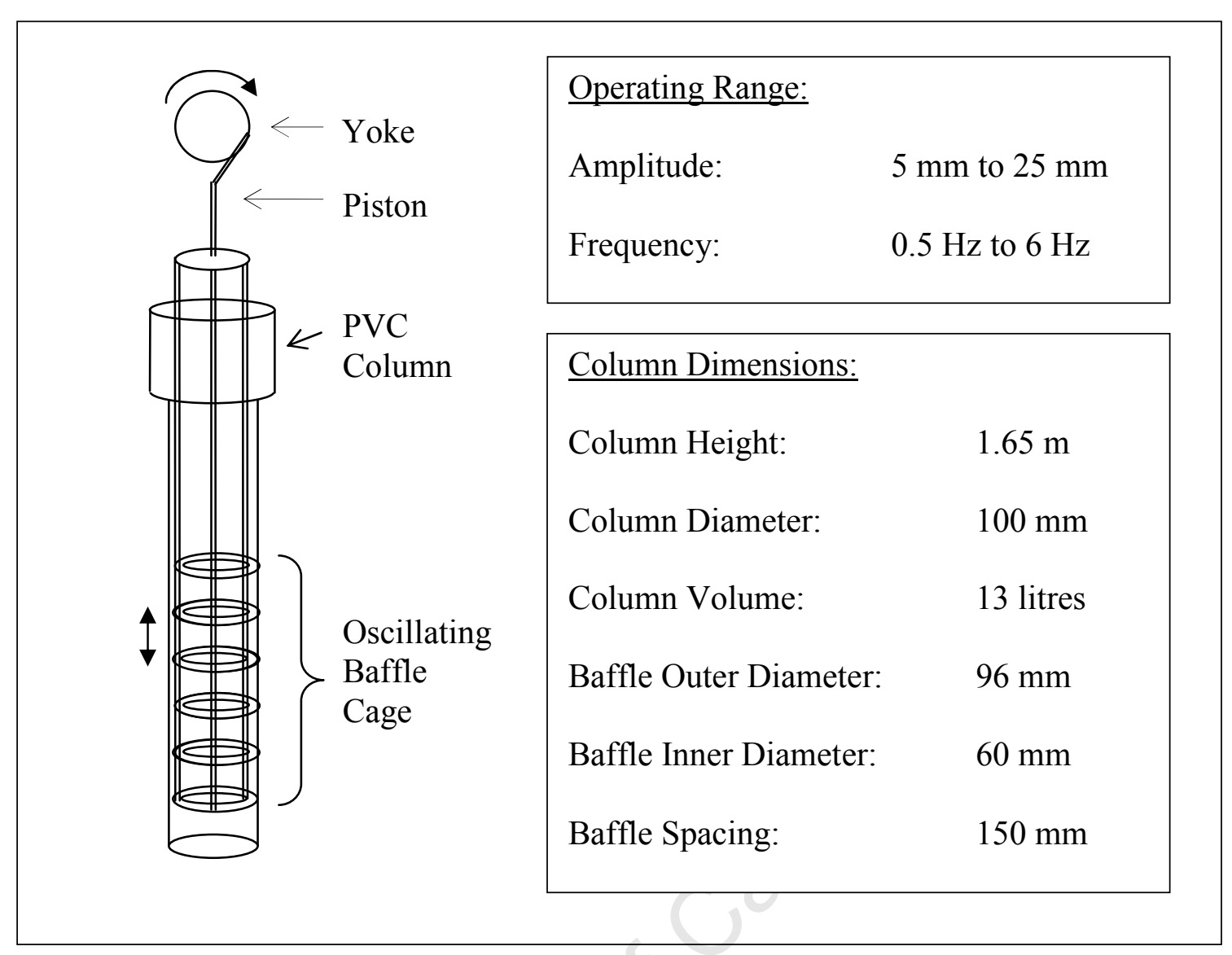

Figure 3.1 - Schematic of the Oscillatory Baffled Column Flotation Cell

The baffle spacing and fractional open area are designed to fall within range of the heuristics for optimal mixing given by Harvey et. al. (2001). The range of dimensionless groups is shown in the following table:

Table 3.1: Dimensionless Groups of OBC Design (Refer Table 2.2)

\begin{tabular}{|l|l|}
\hline Oscillatory Reynolds' Number: & $3080 \leq \mathrm{Re}_{o} \leq 46250$ \\
\hline Strouhal Number: & $0.3 \leq S t \leq 1.6$ \\
\hline Baffle Spacing: & $(L / D)=1.5$ \\
\hline Baffle Free Area: & $S=0.44$ \\
\hline Power Input & $0.6 \mathrm{~W} / \mathrm{m}^{3} \leq \bar{\varepsilon} \leq 1682 \mathrm{~W} / \mathrm{m}^{3}$ \\
\hline
\end{tabular}


This range of oscillatory Reynolds numbers is in the upper range of oscillatory Reynolds numbers compared with other OBC studies and is well above the axisymmetric regime of flow $\left(\operatorname{Re}_{\mathrm{o}}<1600\right)$. This high range of $\mathrm{Re}_{\mathrm{o}}$ enables the column to operate within range of the power dissipation values of conventional flotation cells. The Strouhal number is also fairly low compared with most studies. The larger amplitude to column diameter ratio allows for greater power inputs as well as greater eddy propagation through the fluid, resulting in a more uniform energy dissipation rate through the system than would be achieved at lower amplitudes.

For this experimental set-up, the agitated volume of the cell was estimated as being half a baffle spacing above and below the highest and lowest baffle respectively, giving a total agitated volume of 7.07 litres.

\subsubsection{Process Flow Diagram}

The column is set up in continuous operation in order to allow steady-state conditions and provide a more realistic indication of the cell performance in industrial applications. A flow diagram of the apparatus is given in Figure 3.2.

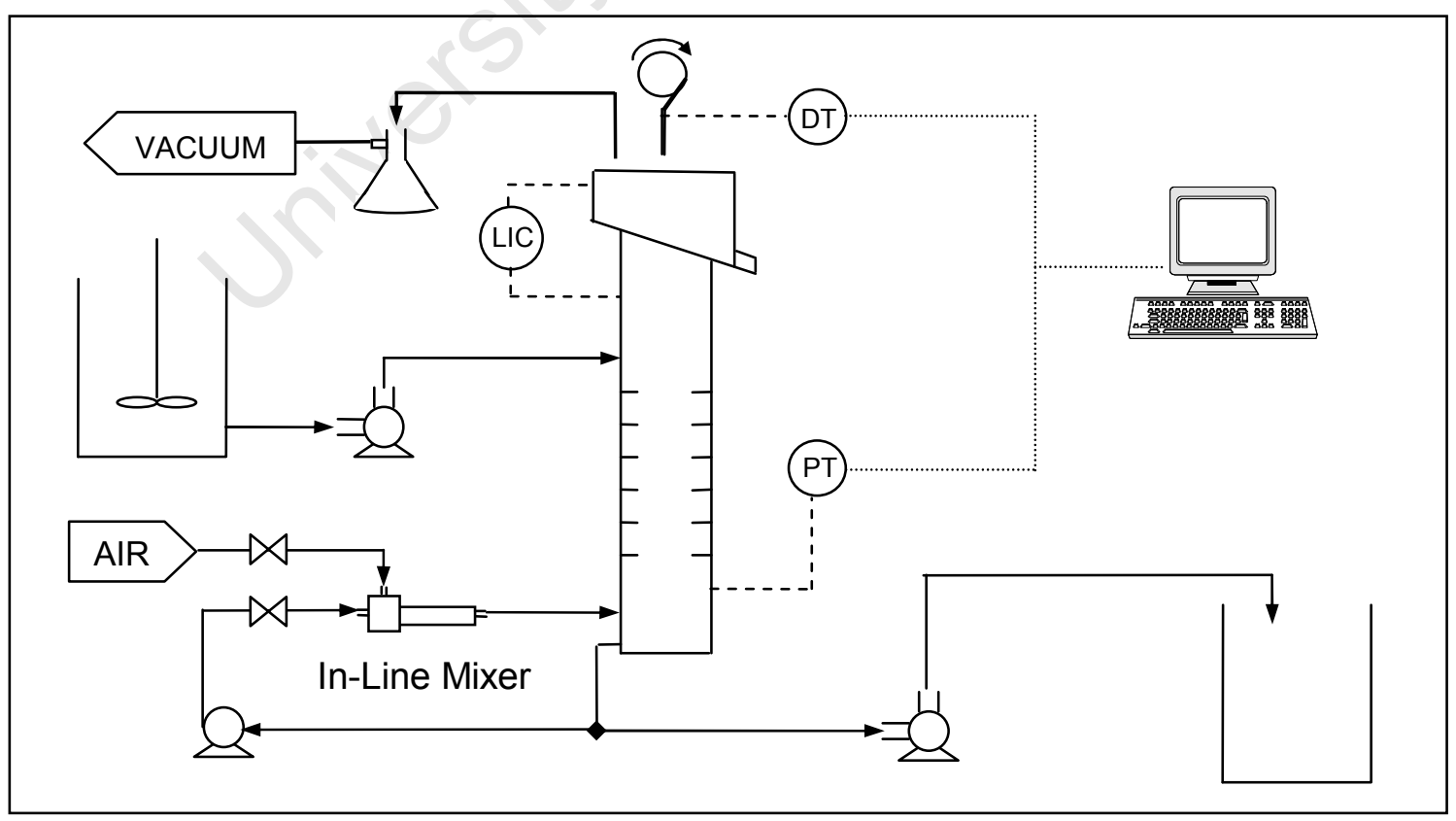

Figure 3.2 - Flow Diagram of Experimental Apparatus 
Slurry is fed continuously from a 550 litre feed tank to a point above the baffle cage and is withdrawn from the base of the column. In order to minimise froth effects on the overall recovery, concentrate is vacuumed continuously during sampling from a few millimetres above the pulp-froth interface using a froth suction system. This technique was also used by Ahmed \& Jameson (1985) and Deglon (1998).

\subsubsection{Bubble Generation System}

Bubbles are generated in the column by means of a static in-line mixer as used in the micro-bubble flotation column (Yoon \& Luttrell, 1989) and the JKMRC High Bubble Surface Area Flux Flotation Cell (Vera et. al., 1999). This apparatus produces bubbles by drawing slurry from the base of the column and pumping it through the in-line mixer. The mixer consists of two concentric steel tubes; a larger outer tube carrying the slurry and a smaller inner tube carrying the air. The inner tube is perforated and covered by porous rubber tubing. Air is introduced at pressure and sparged radially into the passing slurry where it is sheared into a swarm of fine bubbles by turbulent eddies in the slurry. The resulting air-slurry mixture is reintroduced to the base of the column through a distribution nozzle. Air flow and slurry recycle rate are controlled by throttle valves upstream of the static mixer. The final bubble size distribution is controlled by the air flow rate, the recycle flow rate and the frother concentration in the slurry.

\subsubsection{Level Control}

Level control is achieved by a potentiometric system, consisting of 2 Nickel-Chrome wire electrodes, each $1 \mathrm{~m}$ in length, mounted on the inside of the column. A potential difference is applied across one of the wires and the resulting potential difference of the second wired is then measured. This system generates a linear output (0 to $4 \mathrm{~V}$ ) proportional to the level of the froth/liquid interface. This signal is sent to a PI controller which adjusts the speed of the tails pump accordingly to maintain the desired set point. Although the measurement is sensitive to $\mathrm{pH}$ and conductivity in solution, in practice the system is able hold a desired set point for an extended period of time. 


\subsubsection{Pressure and Displacement Measurement}

The column is fitted with a Wika S-11 pressure transducer for the measurement of the pressure drop across the baffle cage and a Temposonics R-Series displacement transducer to measure the displacement of the baffle cage with time. These two measurements are used to calculate both the power input and the gas hold-up in the cell during flotation tests (see section 3.2 for details of calculation). The pressure transducer is mounted in a specialized port at the base of the column one baffle spacing below the lowest baffle in the column. The displacement transducer is fixed onto the mounting of the column and attached to the piston by a custom-made brace. The sensors each produce a $4-20 \mathrm{~mA}$ output signal which is amplified and sent through a set of low pass filters in order to eliminate high frequency noise. The signals are then transferred to an analogue-to-digital card from where they can be read using the computer software, LabView@.

\subsubsection{Bubble Size Measurement}

Bubble size distributions are measured using the UCT Bubble Analyser. The column is fitted with two custom-made ports for the bubble analyser capillary allowing bubbles to be measured at two different positions in the column, viz. the centre of the lowest baffle and the centre of the $5^{\text {th }}$ baffle from the bottom. The method of using this type of bubble size measurement is well documented (Randall et. al., 1989; Tucker et. al., 1994).

\subsection{Hydrodynamic Characterisation}

In order to inform the interpretation of flotation data, tests were first performed to evaluate the effect of the $\mathrm{OBC}$ operating conditions on key variables which are thought to affect flotation. Of particular importance to this study was the characterisation of power dissipation in the $\mathrm{OBC}$ since power dissipation is a key parameter in determining the hydrodynamic environment for particle-bubble contacting. The effect of operating conditions on factors such as bubble size, gas hold-up and mixing was also evaluated since fundamental flotation models predict that these variables have an impact on 
flotation kinetics. This information was then used to inform the interpretation of flotation data in chapters 5 and 6 .

The characterisation was performed in two stages, with the ungassed power dissipation, bubble size and the mixing characteristics being investigated prior to flotation in wateronly operation, and the gassed power dissipation and gas hold-up being measured during flotation testing. The following section describes the experimental methods and data analysis procedures that were used during this test work.

\subsubsection{Characterisation of Power Dissipation}

Since one of the key focuses of the study is the role which power dissipation plays in promoting particle-bubble contacting in the $\mathrm{OBC}$, it was essential to fully characterise the power behaviour of the system as a function of its operating variables. This information could then be used to calculate the average power dissipation in the cell during flotation testing.

The power dissipation in the $\mathrm{OBC}$ was calculated using the method first derived by Hafez \& Prochazka (1974). This method makes use of a momentum balance over the plate stack where the power is given by the product of the instantaneous force of the baffles on the fluid and the velocity of the baffle cage. The theory behind this analysis is detailed in Section 2.2.5. To achieve the measurement, pressure and displacement signals were recorded experimentally both prior to flotation in a water-only system and during each flotation test. The data was then exported to Matlab where it was fitted with the following equations:

$$
\begin{aligned}
& D(t)=-x_{o} \cdot \cos (\omega \cdot t+\delta) \\
& P(t)=\Delta P_{i, \text { max }} \cos (\omega \cdot t+\delta)+\Delta P_{f, \text { max }} \sin (\omega \cdot t+\delta) \cdot|\sin (\omega \cdot t+\delta)|
\end{aligned}
$$


where $\Delta \mathrm{P}_{\mathrm{i}, \max }$ and $\Delta \mathrm{P}_{\mathrm{f} \text {,max }}$ are the maximum inertial and frictional pressure drops respectively and $\delta$ is a lag term to account for differences between the start of the cycle and the start of the measurement. This equation assumes that the pressure is instantaneously transmitted from the baffles to the base of the column with negligible lag time inbetween. The validity of this assumption for high gas hold-up is discussed in section 4.1.2.2.

To calculate the instantaneous power input, the fitted pressure signal was multiplied by the cage velocity as per the following equation (Hafez \& Prochazka, 1974):

$$
J=\left(\frac{\pi D^{2}}{4}\right) \cdot \Delta P \cdot x_{o} \cdot \omega \cdot \sin (\omega \cdot t+\delta)
$$

where $\mathrm{J}$ is the instantaneous power input to the fluid, $\Delta \mathrm{P}$ is the pressure drop across the baffle cage and $x_{0} \cdot \omega \cdot \sin (\omega \cdot t+\delta)$ is the calculated velocity of the baffle cage. Since the power oscillates with time, the time-averaged power dissipation is given by:

$$
J_{\text {ave }}=\frac{\int_{0}^{T} J \cdot d t}{\int_{0}^{T} d t}
$$

The resulting time-averaged power inputs were compared with various models from the literature in order to validate the measurement technique. Correlations were also drawn up to predict future power inputs. The results of this comparison are detailed in Section 4.1.1.

\subsubsection{The Effect of Power Dissipation on Bubble Size}

As detailed in Section 2.3.3, bubble size has a significant impact on all the subprocesses on flotation, including the collision rate, probability of collision and 
probability of detachment. Since the focus of this study is on the effect of hydrodynamic parameters such as power dissipation and viscosity on flotation kinetics, independent of the effect of bubble size, it was necessary to assess the effect of power dissipation on the bubble size in the agitated zone. Ideally, it would be best to obtain a constant bubble size distribution with changing power dissipation as this would mean that changes in flotation kinetics in the cell could be directly attributed to changes in the hydrodynamic environment rather than changes in gas phase characteristics. The aim of this test work was therefore to evaluate the extent to which bubble break-up had been mitigated in the agitated zone of the cell.

The effect of power dissipation on bubble size was investigated by measuring the bubble size using the top bubble sizing port (i.e. the centre of the $5^{\text {th }}$ baffle from the bottom). The bubble size was first quantified without baffles and appropriate static mixer operating settings were selected so as to provide a consistent and fine bubble size distribution which would be relatively insusceptible to further bubble break-up in the agitated zone. The final static mixer settings chosen were an air rate of $2 \mathrm{l} / \mathrm{min}$, a recycle rate of $13 \mathrm{l} / \mathrm{min}$ and a frother dosage of $10 \mathrm{ppm}$ Dowfroth 250 . These settings were found to produce a mean bubble size of $0.62 \pm 0.03 \mathrm{~mm}$, with the following bubble size distribution: 


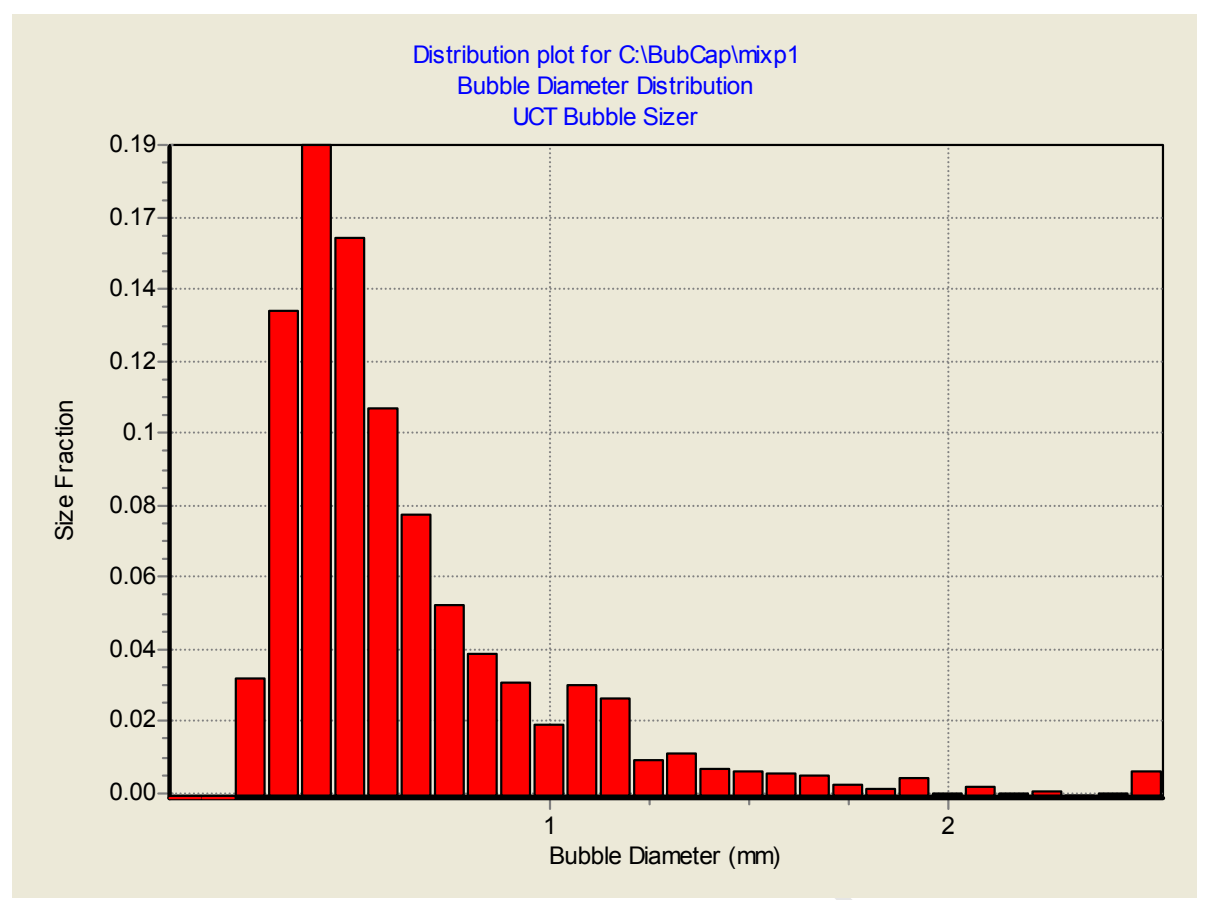

Figure 3.3 - Bubble Size Distribution Produced by the Static In-line Mixer.

(Frother Dosage $=10 \mathrm{ppm}$, Recycle Rate $=13 \mathrm{l} / \mathrm{min}$, Air Flow Rate $=2 \mathrm{l} / \mathrm{min}$ )

These settings correspond to a superficial gas velocity $\left(\mathrm{J}_{\mathrm{g}}\right)$ of $0.42 \mathrm{~cm} / \mathrm{s}$ and a bubble surface area flux $(\mathrm{Sb})$ of $18.4 \mathrm{~s}^{-1}$. This is low compared with conventional columns which typically operate at $1<\mathrm{J}_{\mathrm{g}}<2 \mathrm{~cm} / \mathrm{s}$ and $20<\mathrm{Sb}<60 \mathrm{~s}^{-1}$ (Vera et al., 1999). However these settings ensure a small bubble distribution which is relatively insusceptible to breakage in the agitated zone.

The baffles were then replaced and the bubble size was evaluated over a range of frequencies and amplitudes so as to produce a wide range of power dissipation levels between 17 and $1682 \mathrm{~W} / \mathrm{m}^{3}$.

\subsubsection{The Effect of Operating Conditions on Gas Hold-Up}

As shown in Section 2.3, the fundamental flotation models predict that the flotation rate is directly proportional to the gas hold-up (c.f. Equations $2.32,2.80 \& 2.81$ ). In order to account for the potential effect of gas hold-up on flotation kinetics, it was necessary to quantify the gas hold-up in the column at each of the flotation conditions tested. Gas 
hold-up was therefore measured as part of the standard operating procedure during flotation tests.

The gas hold-up in the $\mathrm{OBC}$ was calculated using the average pressure measured at the base of the column during flotation testing. This method is commonly used to determine gas hold-up in continuous flotation systems. The addition of gas to the system produced a decrease in the average density of the air-slurry mixture and therefore a decrease in the static pressure measured at the base of the cell. It can be shown that the gas hold-up in the cell is related to the ratio of the static pressure under gassed conditions to the static pressure under ungassed conditions, as shown in the following equation:

$\phi=1-\frac{\overline{\Delta P}}{\rho_{\text {slurry }} \cdot g \cdot h}$

Pressure measurements were recorded during each flotation test and the percent solids in the tails stream was used to calculate the slurry density in the cell. The gas hold-up in the system was then calculated using equation 3.5.

\subsubsection{The Effect of Operating Conditions on Mixing}

As shown in Section 2.2, OBC's are able to produce good plug flow conditions at low frequencies and amplitudes and good global mixing characteristics at high frequencies and amplitudes. It was thought that the change in mixing characteristics may have an effect on the overall recoveries obtained within the cell. In order to investigate the flotation kinetics independently of mixing effects, it was therefore necessary to quantify the nature of the mixing in the cell as a function of the cell operating conditions and the sensitivity of the flotation kinetics to mixing effects. These tests were performed under the same operating conditions as those used during the moderate hydrophobicity flotation tests (c.f. Table 3.3). This information could then be used to account for the mixing effects in the flotation data, allowing an evaluation of the effect of power input on the flotation kinetics more directly. 
The effect of power input on mixing was quantified using the pulse injection method (Danckwerts, 1953). To achieve this, the feed line was injected with a tracer of $20 \mathrm{ml}$ of $100 \mathrm{~g} / 1 \mathrm{NaCl}$ and the conductivity of the tails solution was measured and logged using a FLV90 TPS meter. The resulting conductivity signal was normalised to the area underneath the conductivity curve according to the following formula (Levenspiel, 1962):

$$
E(t)=\frac{C(t)}{\int_{0}^{\infty} C(t) \cdot d t}
$$

where $\mathrm{E}(\mathrm{t})$ is known as the "exit age distribution" and $\mathrm{C}(\mathrm{t})$ denotes the measured conductivity signal. The shape of the $E(t)$ curve was then fitted with a tanks-in-series model, as per the method of Yianatos (2007):

$$
E(t)=\frac{t^{N-1} \cdot e^{-(t \cdot N / \tau)}}{(\tau / N)^{N} \cdot \Gamma(N)}
$$

where $\tau$ is the mean residence time and $\mathrm{N}$ is the equivalent number of perfectly mixed tanks in series. $\Gamma(\mathrm{N})$ is known as the Gamma function and is equivalent to an $(\mathrm{N}-1)$ ! term but is able to account for non-integer solutions of $\mathrm{N}$. The Gamma function is given by the following equation:

$$
\Gamma(N)=\int_{0}^{\infty} t^{N-1} e^{-t} d t
$$

Using these equations, the value of $\mathrm{N}$ in equation 3.7 was calculated for each set of the moderately hydrophobic flotation conditions, as outlined in Appendix B. 


\subsection{Quartz Flotation}

Quartz was selected as the test mineral for flotation in order to benchmark the study against previous experimental studies on power input and flotation kinetics (Breytenbach, 1995, Deglon, 1998, Pyke, 2004, Newell, 2006). The major advantage of using quartz is that it is a homogeneous ore type which does not have any problematic chemical effects, such as oxidation, which would complicate the analysis of flotation data.

Many researchers have used methylation techniques in order to render their quartz particles hydrophobic (Pyke, 2004, Sherrel, 2004, Newell, 2006), however given the large size of sample required in this study, the use of an amine collector was considered to be more appropriate. Two types of amine collector were used in this study; hexadecyl pyridinium chloride (HPYC), as used by Breytenbach (1995) and Deglon (1998), and dodecylamine (DDA) which is a common amine collector which has been well characterised in terms of its adsorption behaviour (Smith \& Scott, 1990).

\subsubsection{Ore Sample}

Since the primary aim of the study was to evaluate the $\mathrm{OBC}$ as a novel cell for fine particle recovery, a relatively fine grind size of sub 106 microns was selected for the flotation test work. A $300 \mathrm{~kg}$ sample of No. 2 Foundry Sand $\left(>99.6 \% \mathrm{SiO}_{2}\right)$ was obtained from Consol Industrial Minerals in Cape Town. The sample was milled in a $0.42 \mathrm{~m}$ diameter continuous ball mill, which was operated in closed circuit with a 106 micron screen to provide a product with the following particle size distribution: 


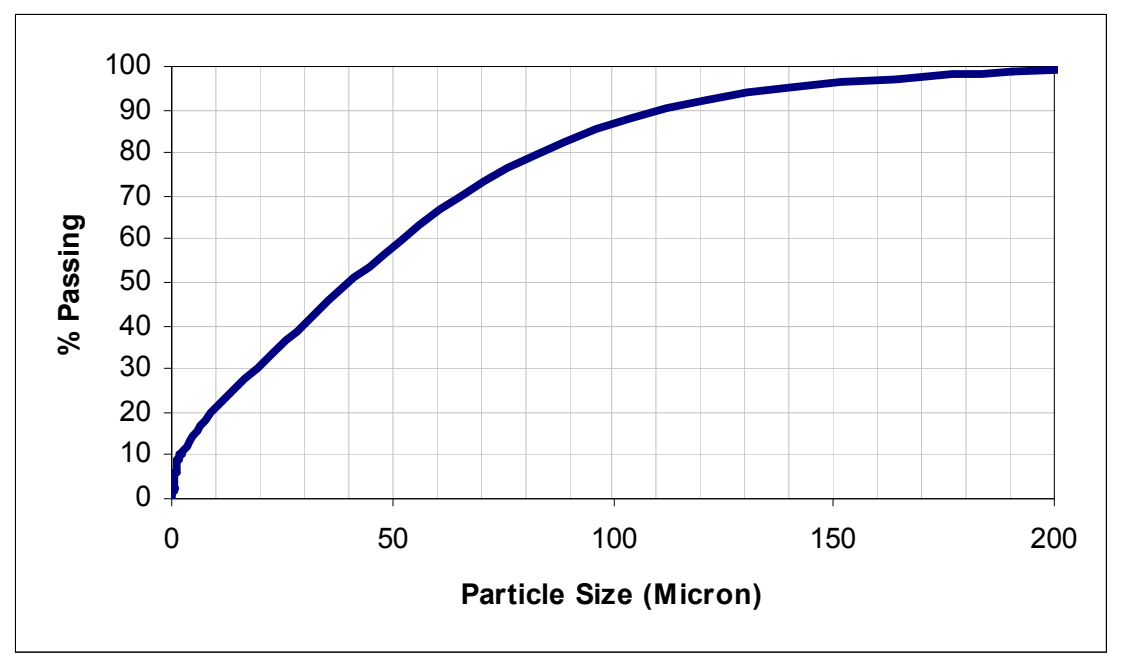

Figure 3.4 - Quartz Particle Size Distribution

Particle size was measured using a Malvern Mastersizer ${ }^{\mathrm{TM}}$ to give an accurate indication of particle size at fine particle sizes. This method uses laser diffraction to determine the particle size and therefore gives a slightly larger reading than that obtained from a screen.

As shown in Figure 3.4, the sample contained $50 \%$ passing 40 micron material and thus contained a large fraction of fine material.

After milling, the sample was calcined in a large pottery kiln at $600{ }^{\circ} \mathrm{C}$ for 8 hours in order to remove any organic material on the surface of the quartz. Many researchers have also utilised acid washing techniques to further cleanse the quartz surface of any inorganic material prior to flotation. However, previous work by Breytenbach (1995) indicated little added effect of acid washing on the floatability of No. 2 Foundry Sand if the sample had been calcined. For this reason, acid washing was not considered to be necessary for the pre-treatment of the quartz.

\subsubsection{OBC Operating Procedure}

Slurry was first made up in the 550 litre feed tank. The tank was filled with a known volume of water and dosed with an appropriate mass of amine collector before the 
solids were added. The $\mathrm{pH}$ was carefully controlled at an appropriate level using $\mathrm{HCl}$ and $\mathrm{NaOH}$ as the $\mathrm{pH}$ modifiers. The slurry was allowed to condition for $10 \mathrm{~min}$ before $10 \mathrm{ppm}$ of Dowfroth 250 was added. Slurry was then conditioned for a further $5 \mathrm{~min}$ before flotation testing began.

At start-up, the OBC was filled with water and the air flow and recycle pump turned on and set to the appropriate level. A feed sample was taken from the feed line before the slurry was pumped into the $\mathrm{OBC}$ at the selected feed rate. The level controller was set to an appropriate conductivity setting such that a froth depth of $10 \mathrm{~mm}$ was obtained. The rig was then allowed to operate for 3 residence times in order to achieve steady state before samples were taken. Concentrate samples were vacuumed directly from the pulpfroth interface into a conical flask for a period of 1.5 minutes. Simultaneously tails samples were collected for a period of 2.5 minutes and the displacement and pressure signals were recorded for power and gas hold-up determination. The amplitude and frequency were then varied appropriately and the column was allowed to regain steady state for 1.5 residence times. Using this technique, the feed tank provided sufficient volume to test 3 different operational conditions per tank before the impeller began to entrain air into the feed tank. The feed to the column was then switched off and a second feed sample collected. At this point, the column was then flushed with a water feed to remove any solids from the recycle line before being emptied and shut down.

\subsubsection{Experimental Programme}

The flotation response of quartz in the $\mathrm{OBC}$ was evaluated as a function of frequency and amplitude at two different levels of particle hydrophobicity. Relatively low collector dosages were chosen in order to simulate the response of the slow floating material which would be found in industrial mineral slurries.

The investigation was carried out in two stages. The first set of experiments was conducted at a collector dosage of $1.56 \times 10^{-5} \mathrm{~mol} / 1$ Hexadecylpyridium Chloride (HPYC) and a $\mathrm{pH}$ of 6 which provided the same collector surface coverage as Deglon (1998). This work was performed as a preliminary scoping study, which evaluated the flotation 
response over a broad range of power dissipations and provided a basis for comparison of the $\mathrm{OBC}$ results with results obtained in a stirred system. The flotation trends obtained at this low collector dosage were then evaluated and used to inform the conditions required for a second round of more comprehensive flotation testing. For the second round of testing, a substantially larger collector dosage of $1 \times 10^{-4}$ mol/l dodecylamine at $\mathrm{pH} 7$ was selected in order to investigate the change in response due to increased hydrophobicity. This represents a relatively large collector dosage for the quartz-amine system but is still sufficiently low to remain below the solubility limit of dodecylamine (Smith \& Scott, 1990). While higher hydrophobicities can be obtained using various quartz methylation techniques, it was decided that this range of hydrophobicities would be more appropriate to evaluate the flotation of slow floating particles. The flotation response of these particles was also evaluated over a more focussed range of power dissipations based on the results of the preliminary flotation tests.

Increasing the collector dosage was found to increase the rate of flotation of particles in the column and therefore the overall recovery. To compensate for this, the air rate and feed rate were adjusted in order to maintain overall recoveries in the vicinity of $90 \%$. The mass fraction of solids in the feed was also adjusted in order to maintain an excess of bubbles in the system and thereby maintain first order kinetics. The experimental settings are summarised in Table 3.3: 
Table 3.2: Experimental Conditions for Quartz Flotation Experiments

\begin{tabular}{|c|c|c|}
\hline Setting & $\begin{array}{c}\text { Low } \\
\text { Hydrophobicity }\end{array}$ & $\begin{array}{c}\text { Moderate } \\
\text { Hydrophobicity }\end{array}$ \\
\hline \hline Collector Type & HPYC & Dodecylamine \\
Collector Dosage & $1.6 \times 10^{-5} \mathrm{~mol} / 1$ & $1 \times 10^{-4} \mathrm{~mol} / 1$ \\
$\mathrm{pH}$ & 6 & 7 \\
Air Rate & $11 / \mathrm{min}$ & $0.551 / \mathrm{min}$ \\
$\%$ Solids & $2.5 \%$ & $1.0 \%$ \\
Residence Time & $5.8 \mathrm{~min}$ & $2.8 \mathrm{~min}$ \\
Recycle Rate & $61 / \mathrm{min}$ & $61 / \mathrm{min}$ \\
\hline
\end{tabular}

Using the above settings, a total of 13 tests were conducted at the low hydrophobicity settings and 23 tests at the moderate hydrophobicity settings. Tests were conducted at amplitudes of 5, 10 and $25 \mathrm{~mm}$, and frequencies between 0.5 and $5.3 \mathrm{~Hz}$ in order to produce power dissipations between 2.0 and $1010 \mathrm{~W} / \mathrm{m}^{3}$. For the moderate hydrophobicity settings, a more focussed power dissipation range of 0 to $200 \mathrm{~W} / \mathrm{m}^{3}$ was selected based on the findings of the weakly hydrophobic flotation tests. Further detail of the experimental conditions used during flotation tests is given in Appendix B.

\subsubsection{Calculation of Entrainment}

Entrainment in the OBC was accounted for using a similar approach to Deglon (1998), where the mass entrained is given by the following equation:

$$
\dot{m}_{i, \text { entrained }}=x_{e} \cdot C_{i, p u l p} \cdot \dot{V}_{\text {conc }}
$$

where $\dot{m}_{i, \text { entrained }}$ is the mass flow rate of size class $\mathrm{i}$ which is recovered to the concentrate by entrainment. $\mathrm{C}_{\mathrm{i} \text {,pulp }}$ is the concentration of size class $\mathrm{i}$ in the pulp and $\dot{V}_{c o n c}$ is the volumetric flow of water in the concentrate. $\mathrm{x}_{\mathrm{e}}$ is the entrainment fraction 
which was measured experimentally by Deglon (1998) by conducting collector-less flotation tests. It can then be shown that the recovery by true flotation is given by:

$R_{i, \text { true }}=R_{i}-x_{e} \cdot \frac{C_{i, \text { tail }}}{C_{i, \text { feed }}} \cdot R_{w}$

where $R_{i}$ is the total recovery of size fraction $i$ and $R_{w}$ is the recovery of water to the concentrate.

In order to obtain the entrainment factor, $\mathrm{x}_{\mathrm{e}}$, the entrainment curve obtained by Deglon (1998) for sub-100 micron quartz, using the same froth suction technique, was used. This data was then fitted using the following equation adapted from Savassi et. al. (1998):

$$
\begin{aligned}
& E N T_{i}=\frac{1.54}{\exp \left(2.292\left(x_{i} / \xi\right)^{A D J}\right)+\exp \left(-2.292\left(x_{i} / \xi\right)^{A D J}\right)} \\
& A D J=1+\frac{\ln \left(\delta_{e}\right)}{\exp \left(x_{i} / \xi\right)}
\end{aligned}
$$

where $\mathrm{x}_{\mathrm{i}}$ is the particle size in microns and $\delta_{\mathrm{e}}$ and $\zeta$ are fitted parameters calculated as 0.72 and 138.0 respectively. The fit obtained is shown in Figure 3.5: 


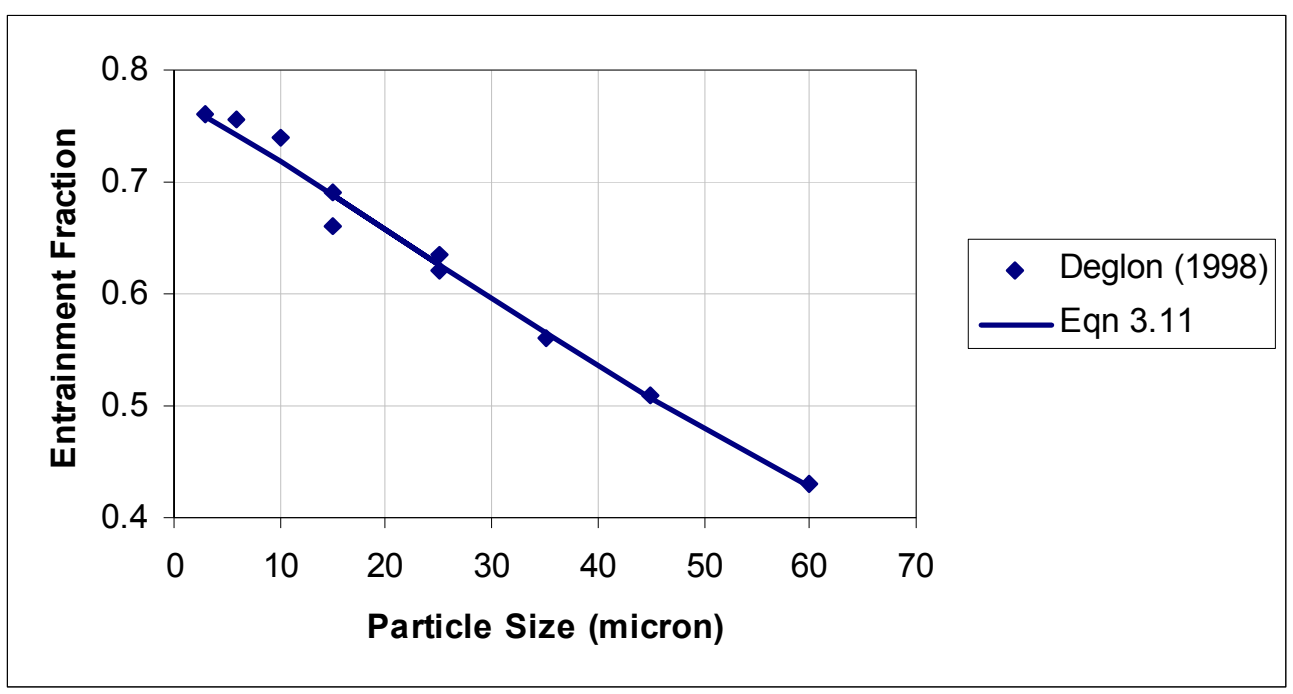

Figure 3.5 - Particle Entrainability as a Function of Particle Size (from Deglon, 1998)

The calculated mass recovered by entrainment in each test was found to be small relative to the total mass recovered due to the low percent solids used in each test. In general, entrainment accounted for less than $10 \%$ of the total recovery for the sub 20 micron material and less than $1 \%$ of the recovery of the remaining material.

Since entrainment only accounted for up to $10 \%$ of the overall recovery, a $10 \%$ error in the recovery by entrainment would result in a maximum error of only $1 \%$ in the overall recovery. At a recovery of $71.6 \%$ (the maximum recovery obtained in the 3 to 17 micron size fraction), this translates into a $4.1 \%$ error in the calculated rate constant.

\subsubsection{Calculation of Rate Constant}

Numerous studies have indicated that the OBC is able to produce both enhanced plug flow conditions at low frequencies and amplitudes and good global mixing characteristics under high frequency and amplitude conditions. A sensitivity analysis was carried out to assess the sensitivity of the flotation rate to changes in mixing and the results are shown in Section 4.4. The calculations show that the rate constant is strongly affected by the mixing characteristics if the recovery in the cell is large (in the vicinity of $90 \%$ ). Based on the findings of the preliminary flotation tests at weakly hydrophobic 
conditions, it was decided to incorporate mixing effects into the analysis of the moderately hydrophobic flotation tests. The flotation rate constant for the quartz flotation tests was therefore calculated using the tanks-in-series model to account for changes in mixing conditions as a function of operating conditions, viz. (Levenspiel, 1962):

$k_{i}=\frac{N}{\tau} \cdot\left[\left(\frac{1}{\left(1-R_{i, \text { true }}\right)}\right)^{1 / N}-1\right]$

where $\mathrm{N}$ is the number of tanks-in-series given by equation 3.7.

It should be noted however that the use of the tanks-in-series model assumes that particles in the cell perfectly follow the fluid and therefore have the same residence time distribution. This assumption seems valid for particles with Stokes numbers less than 1 (c.f. Equation 2.35). For quartz particles in a system with a mean energy dissipation rate of $1 \mathrm{~kW} / \mathrm{m}^{3}$, this equates to particles of less than 75 microns (c.f. Figure 2.16).

\subsection{Nickel Ore Slimes Flotation}

After the flotation behaviour of the OBC had been characterised using quartz, the study was extended to an investigation of the OBC's performance in the flotation of a fine, rheologically complex ore slurry. The aim of this test work was to evaluate the OBC's performance as a novel cell for a fine, rheologically complex ore type where it may have the potential for niche application. Furthermore, the independence of the OBC's bulk flow patterns on slurry rheology provided an ideal opportunity to vary the microturbulent environment independently of the bulk flow conditions. It was hypothesised than an investigation of this kind would allow further insights into the effect of the micro-turbulent environment on the flotation kinetics and inform the OBC's efficacy as a research tool. 
The following section details the nature of the ore slurry as well as the experimental methods used during flotation testing. The method of entrainment and rate constant calculation is also described.

\subsubsection{Ore Sample}

The testing of the $\mathrm{OBC}$ as a novel cell and research tool required a fine and rheologically complex industrial ore slurry, in order to evaluate the hypotheses of this study. The rheological complexity of ore slurries usually originates from the gangue minerals present in the ore sample, which may be fibrous or platy in nature. Such behaviour is often found in ores containing serpentinised olivine gangue, which forms minerals such as Lizardite, Antigorite and Chrysotile. These minerals are particularly prevalent in the Komatiitic nickel sulphide ore deposits of Western Australia (Hoatson et. al., 2006). For this reason, a fine mineral slurry from a West Australian Komatiite nickel operation was selected, which is known for the rheologically complex behaviour of its mineral slurries.

The operation is located on the nickel bearing Agnew-Wiluna Greenstone Belt of Western Australia and processes ore containing finely disseminated pentlandite and millerite grains, interstitial to serpentinised olivines, such as Lizardite, Antigorite and Chrysotile. The fibrous nature of the gangue minerals in this ore, particularly Chrysotile, leads to high viscosities and yield stresses. These complicate cell hydrodynamics and are thought to lead to turbulence damping (Schubert, 1999) and even possible cavern formation around impellers (Arratia et. al., 2006) which can be detrimental to gas dispersion in impeller driven cells (Deglon et. al., 2007). In order to facilitate processing, the final mill product is split into 3 size fractions using two stages of hydrocyclones. The overflow from the second stage of hydrocyclones is known as the "slimes" stream and exhibits the greatest rheological complexity given its fine grind size and high volume fraction of Chrysotile fibres. This was the stream used for flotation testing of the $\mathrm{OBC}$. 
During testing, the slimes stream had a feed composition of approximately $0.35 \% \mathrm{Ni}$, $41.0 \% \mathrm{MgO}, 0.44 \% \mathrm{~S}$ and $3.8 \% \mathrm{Fe}$ with the nickel giving an indication of the sulphide minerals and $\mathrm{MgO}$ giving an indication of the phylosilicate gangue material. The particle size distribution of the stream is shown in Figure 3.6:

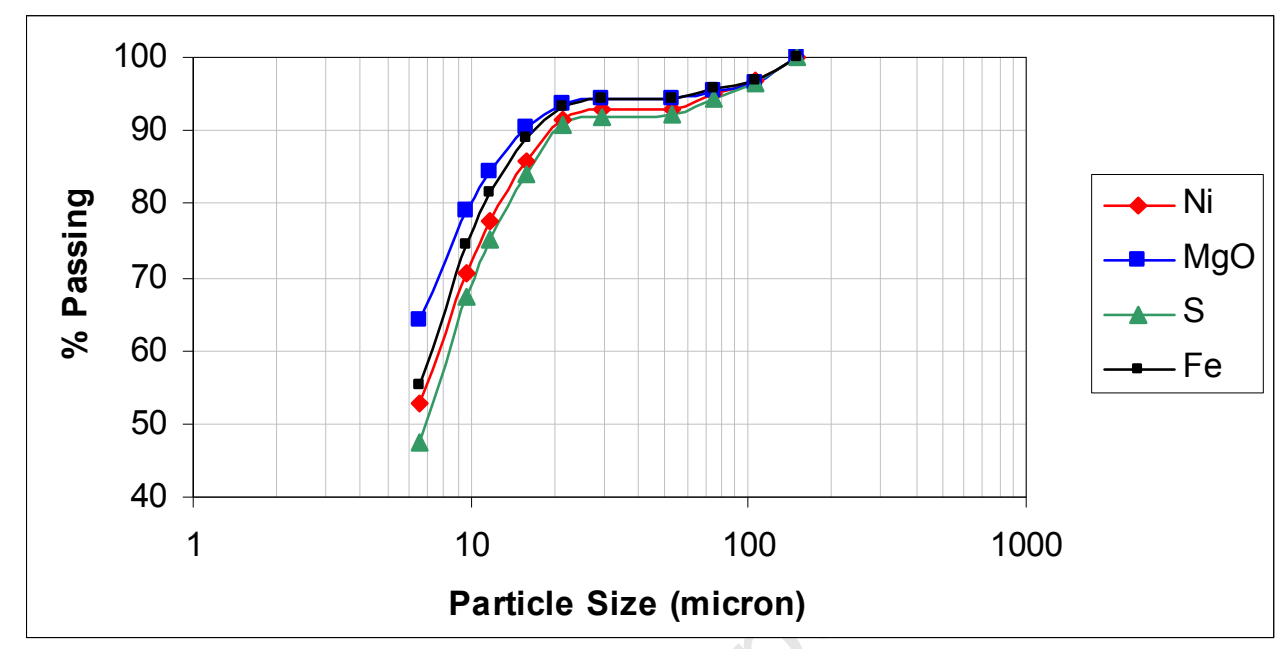

Figure 3.6 - Nickel Ore Slimes Particle Size Distribution

Figure 3.6 shows that the sample was exceptionally fine with a p 80 of approximately 10 microns obtained from cyclosizer results. At this fine grind size, more than $85 \%$ of the nickel sulphide particles are fully liberated of gangue material.

\subsubsection{Sample Rheology}

The fineness of grind in this stream, together with the fibrous nature of the gangue minerals, results in a high yield stress and viscosity of the slurry. The rheology of the stream, together with the percent solids, was monitored over a two week period during flotation tests. Rheology tests were carried out using a Bohlin Visco 88 which consists of a standard cup and spindle apparatus with a volume of $17 \mathrm{ml}$. A known shear rate was then induced on the slurry sample and the resulting torque was measured. The bottom 4 speed settings were not used as the measured torque under these conditions was below the instrument sensitivity of $0.5 \mathrm{mN}$.m. The rheology was first measured on a sample collected directly from the plant, before the sample was diluted using plant 
water and re-measured to obtain a change in the the slurry rheology. Results were fitted with the Bingham plastic model as shown in the following equation:

$\tau_{y}=\mu_{b} \dot{\gamma}+\tau_{b}$

where $\tau_{\mathrm{y}}$ is the shear stress, $\gamma$ is the shear rate, $\mu_{\mathrm{b}}$ is the Bingham viscosity and $\tau_{\mathrm{b}}$ is the Bingham yield stress. Regression lines were found to be statistically linear at greater than the $99 \%$ confidence interval, although better fits were obtained at low percent solids tests than at high percent solids tests. This was due to a slight concave curvature in the high percent solids tests, which led to an increased error in the fitted viscosity and yield stress. For the high percent solids tests, the standard error in viscosity and yield stress was $16 \%$ and $20 \%$ respectively where as the standard error in viscosity for the low percent solids was $3 \%$.

To give an indication of the relative difference in rheological behaviour between settings, the apparent viscosity was also calculated as a function of the shear rate. The apparent viscosity is defined as the ratio of shear stress to shear rate, viz.

$\mu_{a p p}=\frac{\tau_{y}}{\dot{\gamma}}$

This information was however only useful for a qualitative comparison since the exact shear rate distribution within the $\mathrm{OBC}$ as a function of operating conditions was unknown.

\subsubsection{OBC Operating Procedure}

The OBC was set up on site and was operated in semi-batch mode using a 750 litre feed tank in a similar manner to the quartz flotation tests. Slurry for the feed tank was obtained directly from the stage 2 cyclone overflow stream using the in-line shark-fin 
cutter already installed on site. The slurry was gravity fed from the sample cutter to a set of four 250 litre plastic drums and transferred to the feed tank using a pneumatic diaphragm pump.

A 1000 litre tank of process water was also collected from the plant and used to dilute the slurry in the feed tank for the low percent solids test conditions.

Once the slurry had been transferred to the feed tank, a feed sample was collected for analysis by disconnecting the feed line to the OBC and pumping slurry into a sample bucket. The sample was then re-agitated and a $17 \mathrm{ml}$ sub-sample was taken for rheological characterisation.

Slurry was then pumped into the $\mathrm{OBC}$ at a feed rate $5.0 \mathrm{l} / \mathrm{min}$. The column was filled to the lip and the air rate and recycle rate set to $31 / \mathrm{min}$ and $17 \mathrm{l} / \mathrm{min}$ respectively. Based on previous bubble sizing studies conducted in water-only operation, these settings provide a bubble surface area flux of $26.0 \mathrm{~s}^{-1}$. The level controller was then set to an appropriate conductivity setting such that a desired froth depth was obtained. The rig was allowed to operate for 3 residence times in order to achieve steady state. Concentrate and tails samples were collected for 3 and 1.5 minutes respectively although the froth suction technique was not used as this led to excessive entrainment. Instead a constant froth depth of $10 \mathrm{~cm}$ was maintained during flotation tests as preliminary tests had indicated that froth recoveries obtained were of the order of 90 to $100 \%$ at this froth depth. This froth depth was therefore considered to be a good compromise between maximising the froth recovery and minimising the mass of material recovered to the concentrate by entrainment.

Displacement and pressure data was also recorded for power and froth depth determination. Using this technique, the feed tank provided sufficient volume to test six different operational conditions per tank before the impeller began to entrain air into the feed tank. The column was then shut down and a second feed sample collected for assay and rheological characterisation. 
Using the above procedure, approximately 320 litres of slurry remained in the feed tank after testing. The tank was refilled to the 750 litre mark using plant process water in order to dilute the slurry and thereby decrease its yield stress and viscosity. The test procedure was then repeated using the more dilute slurry (+/- $4.5 \%$ solids) and the results compared with the higher percent solids slurry ( $+/-11.2 \%$ solids).

\subsubsection{Experimental Programme}

Preliminary flotation tests were first carried out as part of a broader study in which the cell was operated as a standard column cell. The column was operated on 12 different days and some feed variation was therefore obtained. This information was used as the baseline for comparison of further tests conducted on the $\mathrm{OBC}$ with the baffles inserted.

The effect of power dissipation on the nickel ore slimes flotation rate was then evaluated for power dissipation between 20 and $1330 \mathrm{~W} / \mathrm{m}^{3}$ at an amplitude of $25 \mathrm{~mm}$ using a single tank of feed to prevent any feed fluctuations. Tests were carried out at a percent solids of $11.2 \%$ and $4.5 \%$. Since the xanthate collectors chemisorb onto the surface of the minerals, this change in percent solids is unlikely to have affected particle hydrophobicity.

\subsubsection{Calculation of Entrainment}

The contribution of entrainment to the overall recovery was calculated using a method adapted from Savassi et. al. (1998) where an entrainment factor is defined as follows:

$$
E N T_{i}=\frac{m_{i, \text { entrained }} / w_{\text {conc }}}{m_{i, \text { pulp }} / w_{\text {pulp }}}
$$

where $\mathrm{m}_{\mathrm{i} \text {,entrained }}$ is the mass flow of particles recovered by entrainment, $\mathrm{w}_{\text {conc }}$ is the mass flow of water in the concentrate and $\mathrm{m}_{\mathrm{i}, \text { pulp }} / \mathrm{w}_{\text {pulp }}$ is the concentration of particles of class 
"i" in the pulp just below the pulp-froth interface. For this study, the feed characteristics were used since the feed was injected a few centimetres below the froth zone of the cell.

To calculate the entrainment factor, $\mathrm{ENT}_{\mathrm{i}}$, equation 3.15 is rearranged to yield the following relationship:

$$
m_{i, \text { entrained }}=E N T_{i}\left(\frac{m_{i, \text { feed }}}{w_{\text {feed }}}\right) w_{\text {conc }}
$$

This relationship generates a straight line through the origin when plotting $\mathrm{m}_{\text {entrained }} \mathrm{vs}$. $\left(\mathrm{m}_{\mathrm{i}, \text { feed }} / \mathrm{w}_{\text {feed }}\right) * \mathrm{~W}_{\text {conc }}$ with the slope of the line yielding the entrainment factor. While this is a useful approximation at high water recoveries, several researchers have reported that the true relationship is in fact parabolic below a certain minimum water recovery (Engelbrecht \& Woodburn, 1975, Bisshop \& White, 1976, Lynch et. al., 1981). This leads to overestimation of the mass entrained at low water recovery and therefore an error in the mass recovered by true flotation, particularly in systems where a large proportion of material is recovered by entrainment (as in nickel ore slimes). This concept is illustrated in Figure 3.7:

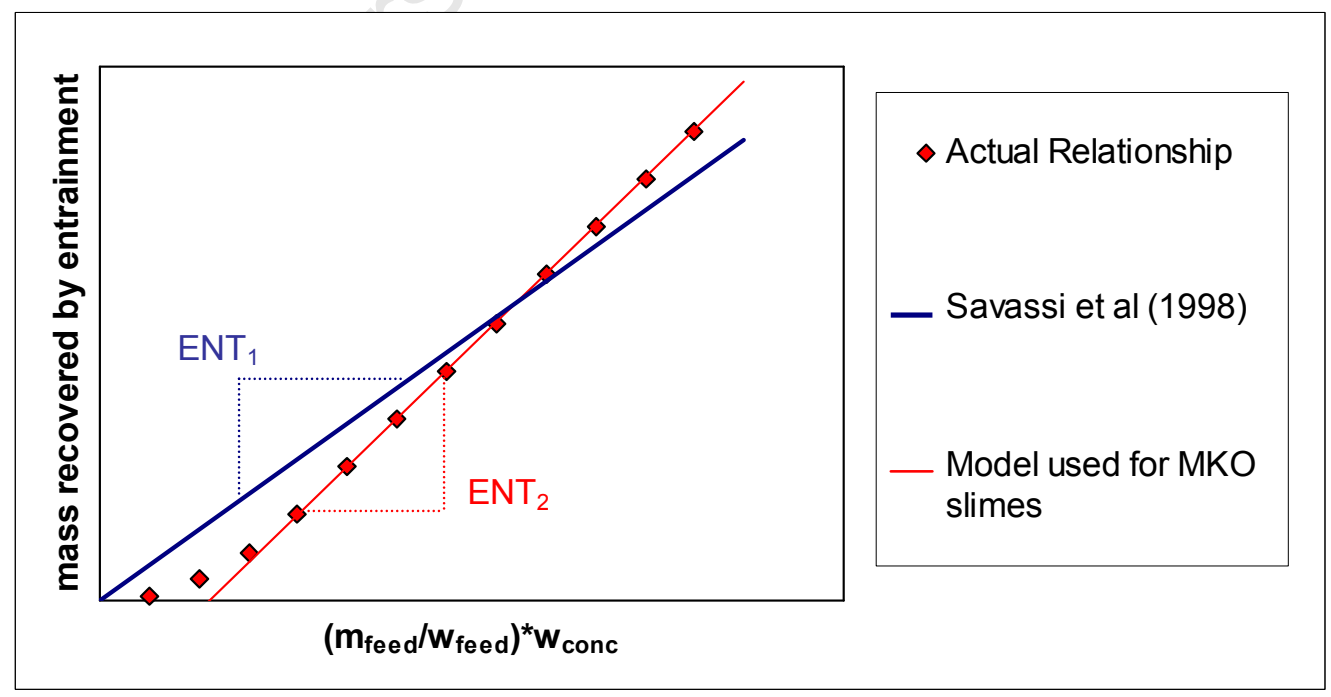

Figure 3.7 - Graph Illustrating the Difficulty in Estimating Entrainment at Low Water Recovery Using the Savassi Model. 
This subtle weakness in the Savassi model when applied to nickel ore slimes led to the need to modify the calculation to improve the entrainment estimate. To do this, it was assumed that the mass vs. water \& pulp density relationship passes through the same xintercept as the total mass of concentrate vs. mass of water \& pulp density relationship. In this way, the calculated mass entrained is always less than the total mass of concentrate and no negative recoveries are calculated. The final entrainment model used is shown in the Figure 3.8.

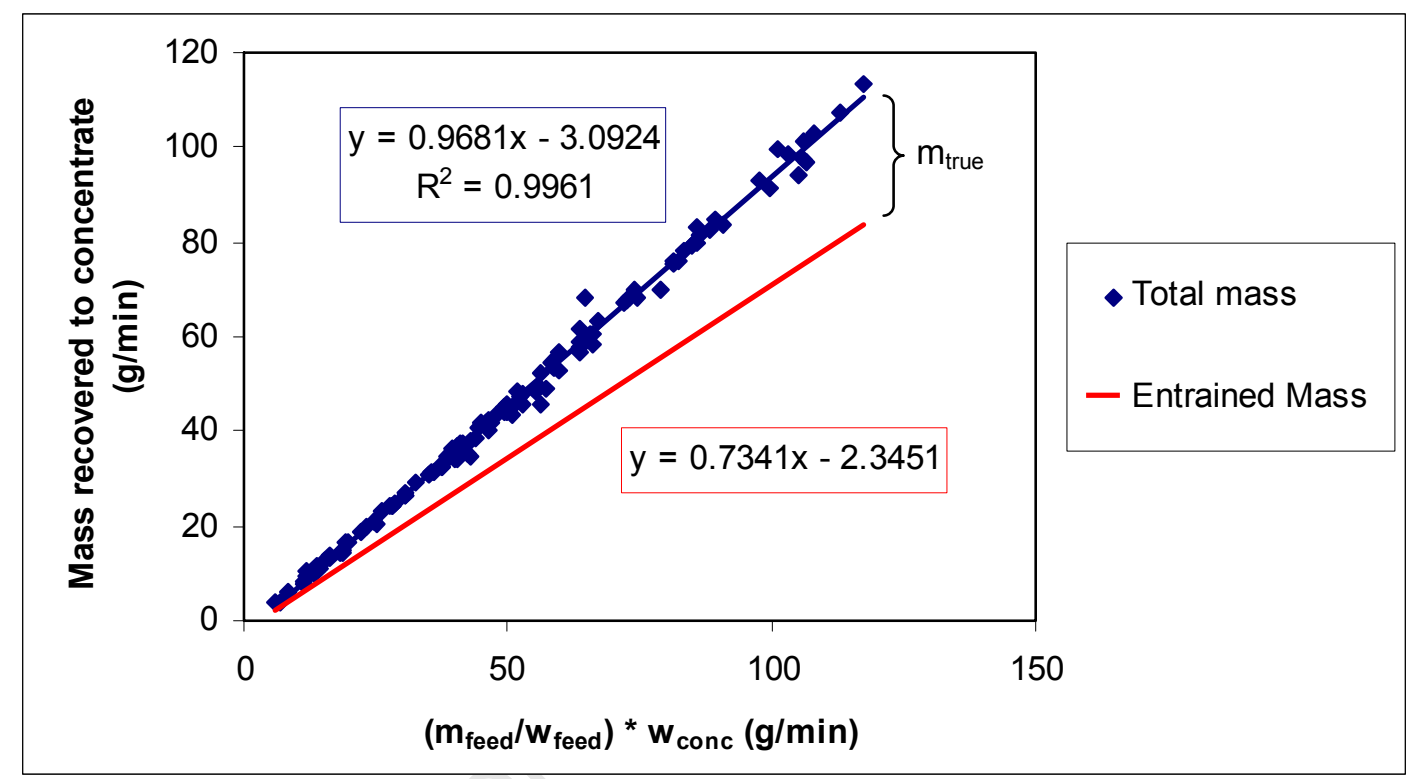

Figure 3.8 - Graph Showing the Modelled Entrainment Mass Flow and the Total Mass Flow in the Concentrate Stream.

Note that in Figure 3.8, both lines pass through the same $\mathrm{x}$-intercept of $3.19 \mathrm{~g} / \mathrm{min}$. This is added as the first constraint on the entrainment curve. A second constraint is then required to obtain the gradient of the entrainment curve (i.e. the ENT factor).

Since the feed did not contain a non-floatable gangue mineral which could be used as a tracer, it was necessary to assume a fraction of floatable gangue in the feed. For the purposes of this calculation, the maximum fraction of floatable gangue was taken to be $7 \%$, corresponding to the maximum recovery of $\mathrm{MgO}$ after 5 minutes obtained during 
previous batch flotation tests. Using this constraint, the slope of the entrainment line could be determined such that the maximum recovery of $\mathrm{MgO}$ by true flotation in any test was no greater than $7 \%$.

This method therefore allowed the mass recovered by entrainment to be calculated based on the mass of water in the concentrate (c.f. Figure 3.8). The mass recovered by true flotation was then obtained by subtracting the mass recovered by entrainment from the total mass in the concentrate.

\subsubsection{Calculation of Rate Constant}

The recovery by true flotation was then obtained using the well known equation for the first-order rate constant in a single CSTR (Levenspiel, 1962):

$$
k=\frac{1}{\tau} \cdot\left(\frac{R_{i, \text { true }}}{1-R_{i, \text { true }}}\right)
$$

It was not considered necessary to use the tanks-in-series model for the nickel slimes flotation data since the recovery in these flotation tests was significantly lower than the quartz flotation tests $(\mathrm{R}<50 \%)$. As will be shown in Section 4.4., the flotation rate constant is not sensitive to changes in mixing conditions for recoveries in this region.

The resulting rate constant was then normalised against the average flotation rate obtained in a standard flotation column to provide a basis for the comparison of results. 


\section{Chapter 4}

\section{Hydrodynamic Characterisation}

This chapter details the effect of OBC operating conditions on key variables which are thought to affect flotation performance. Of particular importance to this study were the power characteristics of the system since power dissipation is a key variable in determining the cell hydrodynamics. The bubble size and gas hold-up were also characterised as these parameters are thought have an effect on the flotation kinetics in terms of the fundamental flotation models. The mixing characteristics of the cell were also investigated since the $\mathrm{OBC}$ system is well known for its enhanced mixing conditions.

\subsection{Power Characterisation}

In Chapter 2 it was shown that power dissipation plays an important role in promoting particle-bubble contacting. Given the OBC's unique agitation mechanism it was necessary to first characterise the cell's power behaviour and validate the observed behaviour against model predictions from the literature. The following section first details the characterisation of power in the $\mathrm{OBC}$ in ungassed operation, before detailing the change in this behaviour when gas is added to the system. 


\subsubsection{Ungassed Power Characteristics}

As discussed in Section 2.2.4, the power characteristics of an OBC differ from those of a conventional cell in that the power input is cyclic and oscillates with time. The overall power input to the cell is given by the sum of an inertial and frictional component of power input (Jealous \& Johnson, 1955). In order to calculate the power input to the cell, the procedure detailed in section 3.2.1 was followed. This analysis uses a curve fitting technique to quantify the magnitude of the inertial and frictional contributions to the power input. An example of the power relationship obtained is shown in Figure 4.1:

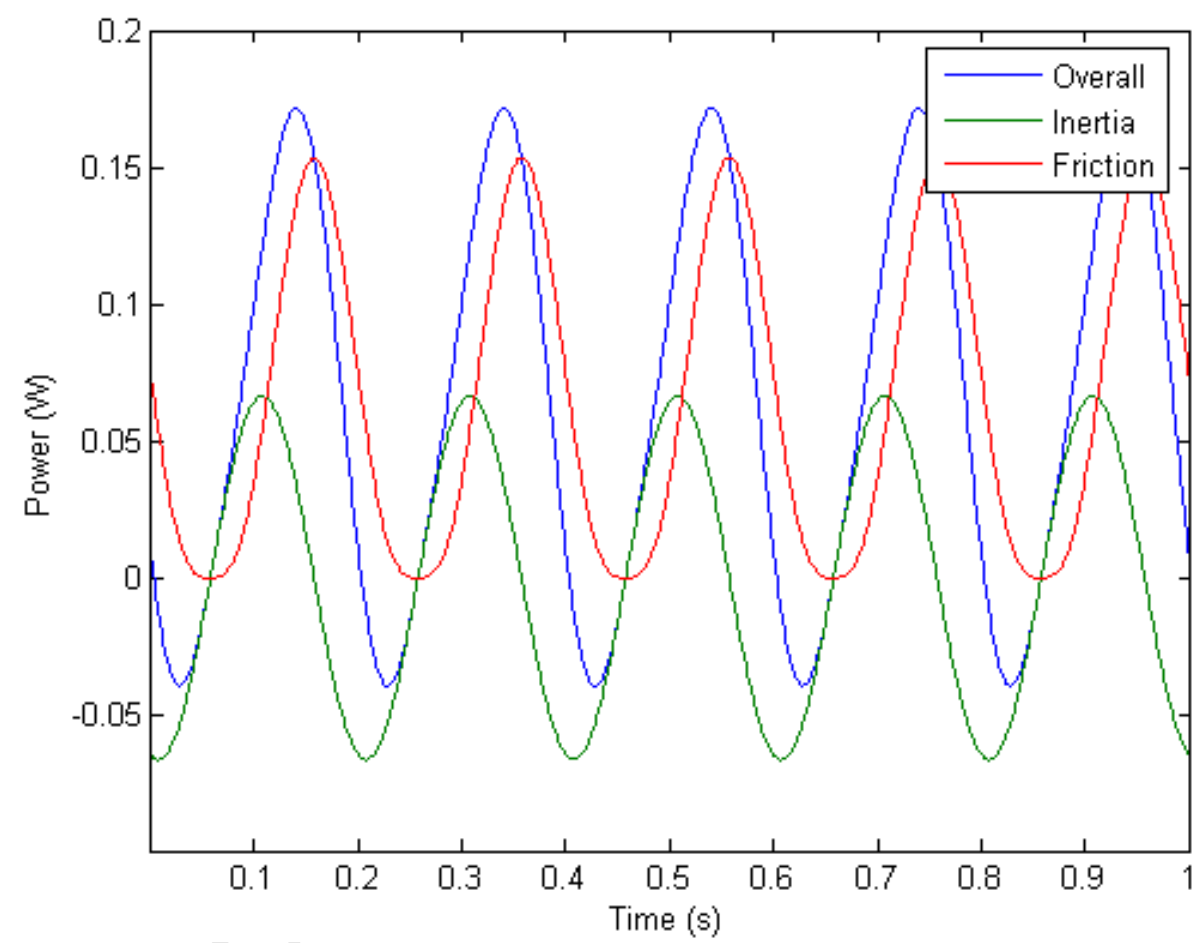

Figure 4.1 - Inertial and Frictional Components of the Overall Power Input for $x_{0}=5 \mathrm{~mm} \&$ $f=2.5 \mathrm{~Hz}$

As can be seen in Figure 4.1, both the frictional and inertial components of power contribute to the total power input. However, the mean contribution of the inertial component is zero. This means that the time-averaged power input to the cell is given by the mean of the frictional component of power and is independent of the inertial component of power input. 
This inertial component of power input is responsible for accelerating the fluid back and forth through the orifice baffles and gives rise to the oscillatory motion of the fluid. This oscillatory motion means that during flotation, particles and bubbles are also continually oscillated back and forth through the orifice baffles, and experience an additional 'oscillatory component' of their velocity. This additional 'oscillatory component' of velocity is large despite the comparatively low power dissipations in the cell. It is speculated that this additional component of velocity may have an effect on the particlebubble contacting rate. This point is discussed further in Chapter 5 when analysing the quartz flotation data.

Interesting to note in Figure 4.1, is that at certain points in the cycle, the overall power input to the system is negative. This phenomenon occurs at a point in the cycle, just after the baffle has reached its maximum velocity. As the baffles start to decelerate, the fluid velocity becomes greater than the baffle velocity and the fluid starts to exert a force on the baffles so as to move them to their maximum or minimum displacement. This is in agreement with the findings of studies on power in oscillatory flow systems (Jealous \& Johnson, 1955, Hafez \& Prochazka, 1974, Baird \& Stonestreet, 1995).

\subsubsection{Ratio of Inertial and Frictional Contributions}

The analysis of the overall power curve also allows a comparison between the relative amplitudes of the inertial power input and frictional power input. The ratio of these two values is equivalent to the ratio of the maximum inertial forces to the maximum frictional forces in the fluid. This ratio is found to be strongly dependent on the Strouhal number (Equation 2.5) as shown in Figure 4.2: 


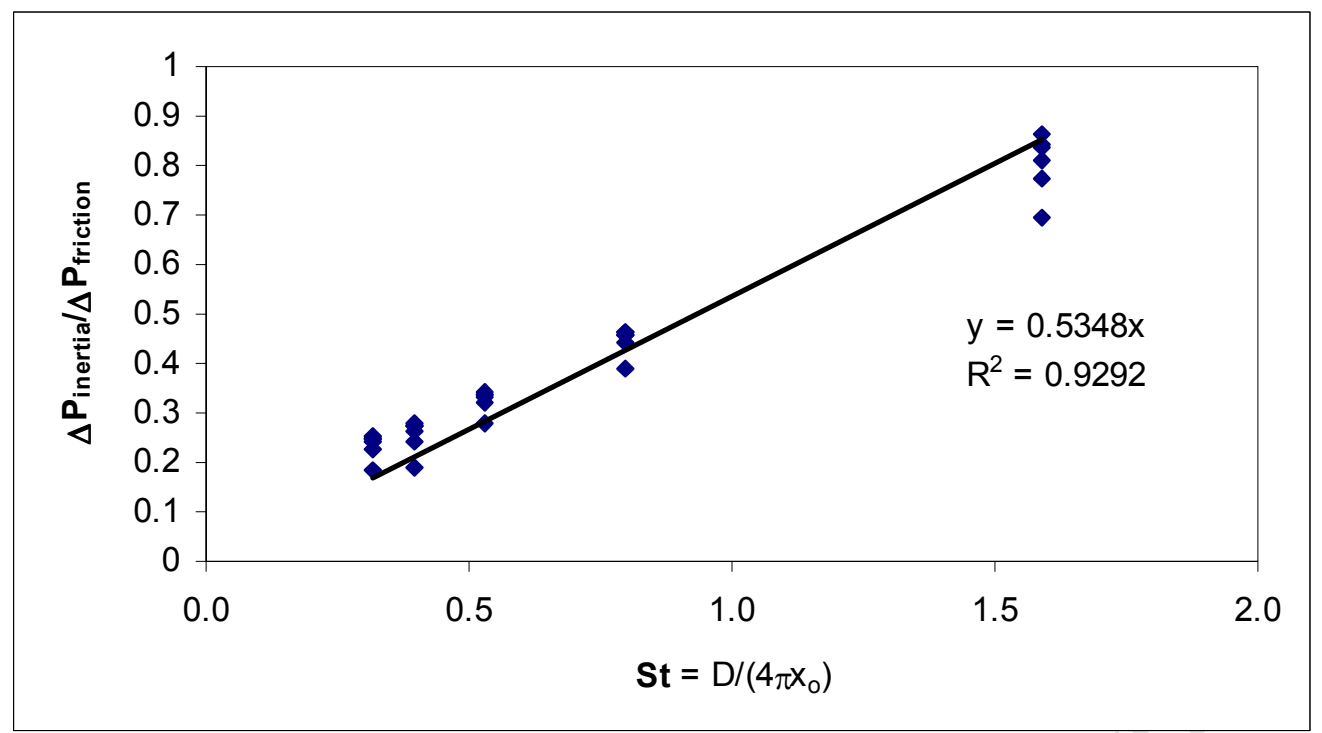

Figure 4.2 - Correlation between Ratio of Inertial to Frictional Forces in the Fluid and the Strouhal Number

Figure 4.2 shows a linear relationship between the ratio of inertial to frictional forces and the Strouhal number (inverse amplitude). This observation was also noted by Hafez \& Prochazka (1974). This relationship arises since in order to produce a desired power input at low amplitude, one must operate at a higher frequency of oscillation. This larger frequency means that the fluid inertia is more rapidly accelerated and decelerated during operation, requiring larger inertial forces at low amplitude (high St). At high amplitude (low St), the oscillation is more gentle and the inertial forces in the fluid are minimised.

In a conventional cell, no inertial forces are present since the bulk motion of the fluid is approximately steady with time. The best approximation of turbulence in a conventional cell is therefore obtained at high amplitude (low $\mathrm{St}$ ) where the inertial component of force is minimised. The possible effect of these forces on the flotation kinetics is further discussed in Chapter 5. 


\subsubsection{Time-Averaged Power Dissipation}

The time-averaged power dissipation to the $\mathrm{OBC}$ was calculated using equation 3.4. Figure 4.3 illustrates the wide range of power dissipation which can be achieved.

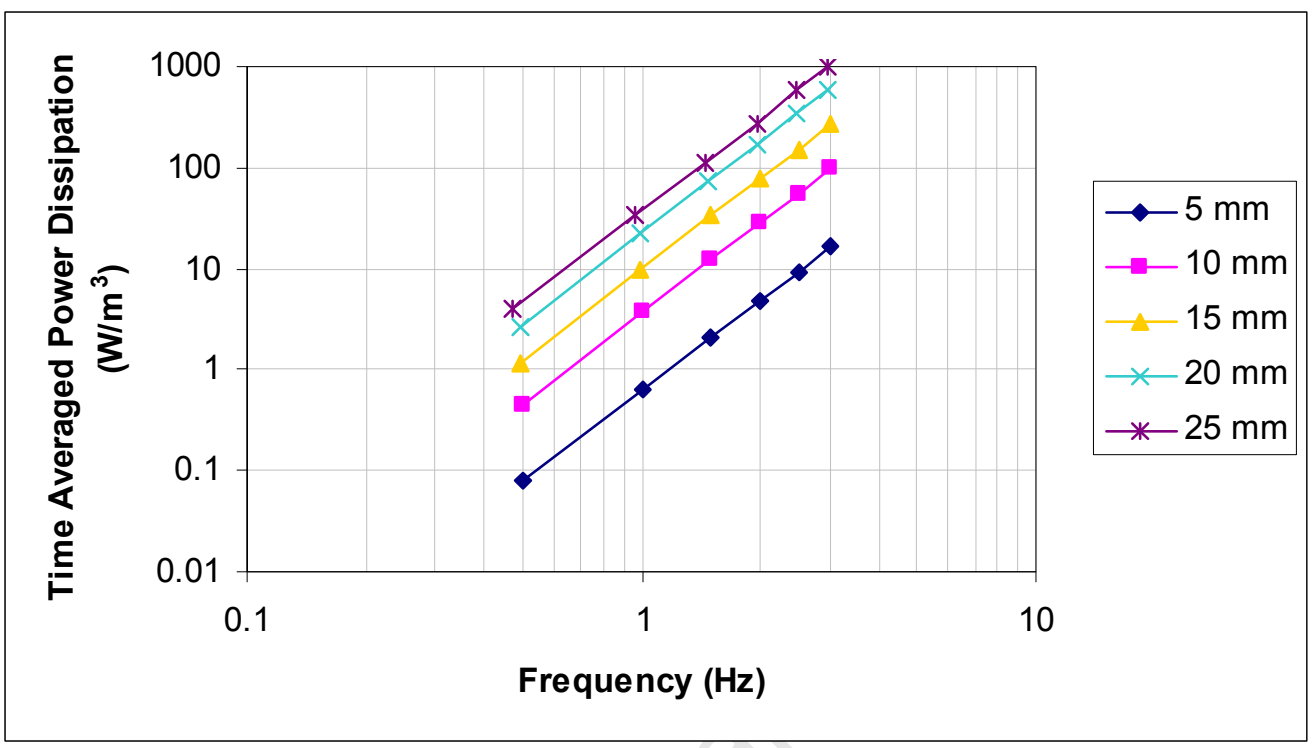

Figure 4.3 - Time-Averaged Power Dissipation in the Agitated Zone of the OBC as a Function of Frequency and Amplitude

Figure 4.3 shows that the time-averaged power dissipation in the $\mathrm{OBC}$ increases exponentially with both frequency and amplitude. Power dissipation in the cell ranges from just $0.64 \mathrm{~W} / \mathrm{m}^{3}$ at $5 \mathrm{~mm}$ and $1 \mathrm{~Hz}$, up to $1010 \mathrm{~W} / \mathrm{m}^{3}$ at $25 \mathrm{~mm}$ and $3 \mathrm{~Hz}$. This constitutes an intermediate power dissipation range since conventional flotation cells range between $700 \mathrm{~W} / \mathrm{m}^{3}$ and $3000 \mathrm{~W} / \mathrm{m}^{3}$ in most applications. The $\mathrm{OBC}$ is also able to run at significantly lower power inputs compared to conventional cells where the gas dispersion and solid suspension sub-processes would otherwise become the limiting processes.

The following two sections compare the results of the time-averaged power dissipation with the theoretical predictions of the two models from the literature; the quasi-steady state model and the acoustic model. This comparison serves to validate the measured 
power behaviour against model predictions and allows the prediction of power dissipation as a function of operating conditions.

\subsubsection{Comparison with the Quasi-Steady State Model}

The quasi-steady state model is given by the following equation (Jealous \& Johnson, 1955):

$\bar{J}_{\text {quasi SS }}=\left(\frac{1}{6}\right) \frac{n \cdot \rho \cdot D^{2} \cdot\left(1-S^{2}\right)}{C_{o}^{2} \cdot S^{2}} \cdot x_{o}^{3} \cdot \omega^{3}$

This model is based on the assumption that the frictional resistance to oscillatory flow can be approximated by the frictional resistance to flow at steady state, and is thus able to predict the power dissipation without using fitted parameters. The derivation of this model is detailed in Section 2.2.5.1.

In order to calculate the frictional resistance, the drag coefficient $\left(\mathrm{C}_{\mathrm{o}}\right)$ in equation 4.1 was first measured by passing a steady state flow through the column and measuring the resulting pressure drop across the baffle cage. The value of $\mathrm{C}_{\mathrm{o}}$ was then calculated using Equation 2.17 and was found to be $0.775 \pm 0.154$. The predicted mean power could then be calculated using equation 4.1 . Figure 4.4 shows a comparison of the experimentally measured power input with the quasi-steady state model predictions. 


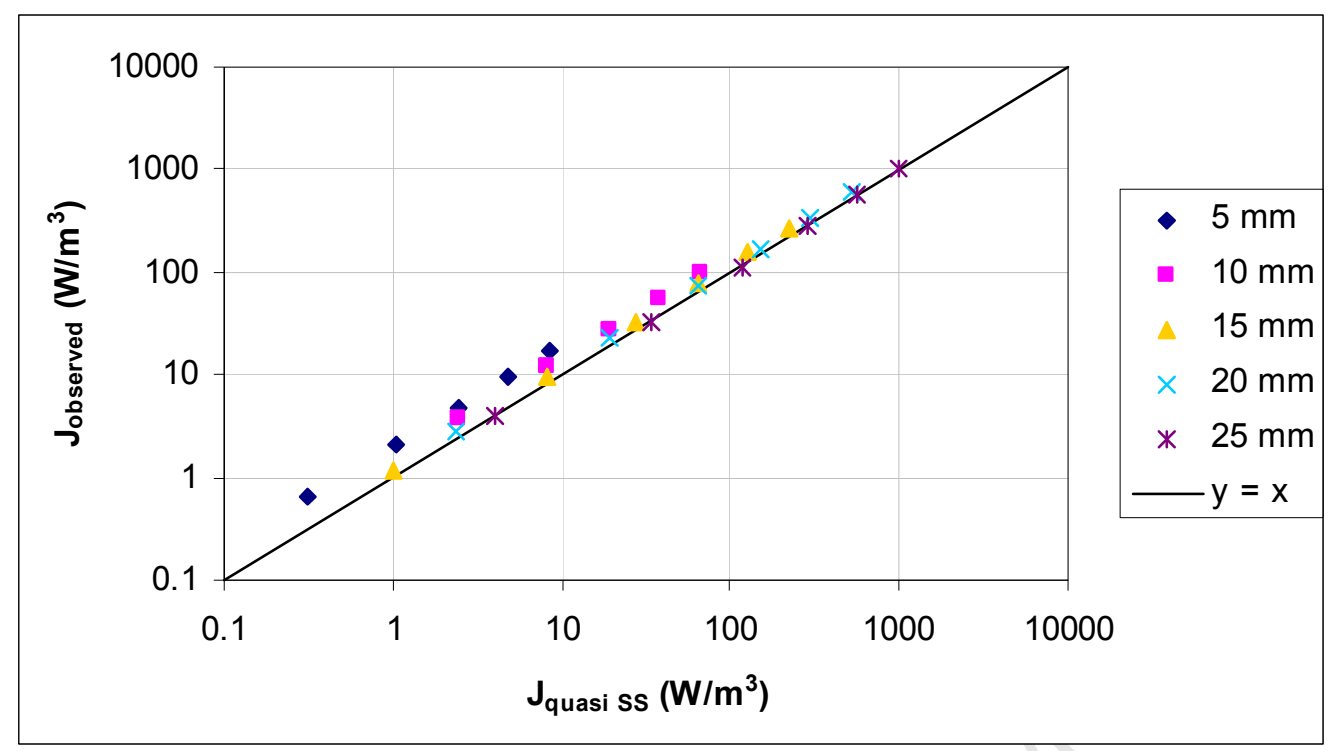

Figure 4.4 - Comparison of Experimentally Determined Power Dissipation with the Predictions of the Quasi-Steady State Model.

Figure 4.4 shows that the quasi-steady state model produces a reasonable prediction of the power dissipation in the $\mathrm{OBC}$, particularly for the higher amplitudes. As the amplitude decreases however, the model starts to under predict the measured power dissipation. For an amplitude of $25 \mathrm{~mm}$, the model prediction is within $4.0 \%$ of the experimentally determined values, but this discrepancy increases up to $109 \%$ for an amplitude of $5 \mathrm{~mm}$. Thus although the quasi-steady state model provides a reasonable approximation for the $25 \mathrm{~mm}$ amplitude, it significantly under predicts the power dissipation for amplitudes of less than $25 \mathrm{~mm}$. This result is consistent with the findings of many previous studies on oscillatory flow, particularly those in which low amplitudes and high frequencies were used (Hafez \& Baird, 1978, Baird \& Stonestreet, 1995).

\subsubsection{Comparison with the Acoustic Model}

The acoustic model is given by the following equation (Baird \& Stonestreet, 1995):

$\bar{J}_{\text {acoustic }}=\frac{\rho \cdot n \cdot D^{2} \cdot l \cdot x_{o}^{2} \cdot \omega^{3}}{S}$ 
This model estimates the frictional resistance as being equivalent to the acoustic resistance of sound waves through an orifice plate, and is thought to be more appropriate for low amplitude, high frequency oscillation (c.f. Section 2.2.5.2). The model makes use of a turbulent mixing length $(l)$, which is believed to be of the order of the column diameter but must be fitted to experimental data. This additional fitted parameter allows significantly more accurate data fits to be obtained, as illustrated by the results in Figure 4.5.

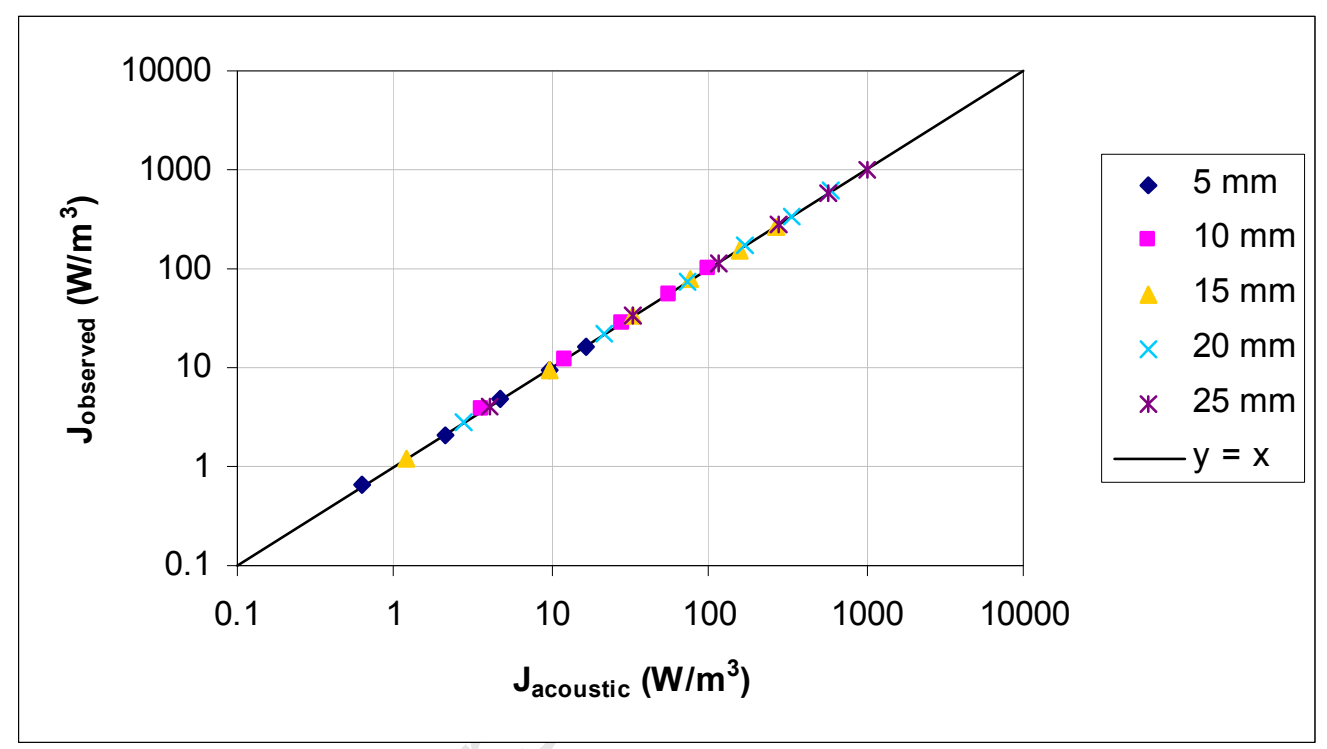

Figure 4.5 - Correlation between Time-Averaged Power Dissipation and the Power Input Predicted by the Acoustic Model.

Figure 4.5 shows that the acoustic model is able to accurately predict the power dissipation behaviour of the $\mathrm{OBC}$ for both high and low amplitudes. The mixing length, $l$, in equation 4.2 is theoretically defined as the average distance travelled by turbulent eddies in the fluid. However, in this case, the mixing length is simply a fitted parameter and may not necessarily be equivalent to the actual displacement of turbulent eddies. The mixing length was however found to correlate well with the inverse of the Strouhal number (c.f. Equation 2.5) due to its link to eddy propagation: 


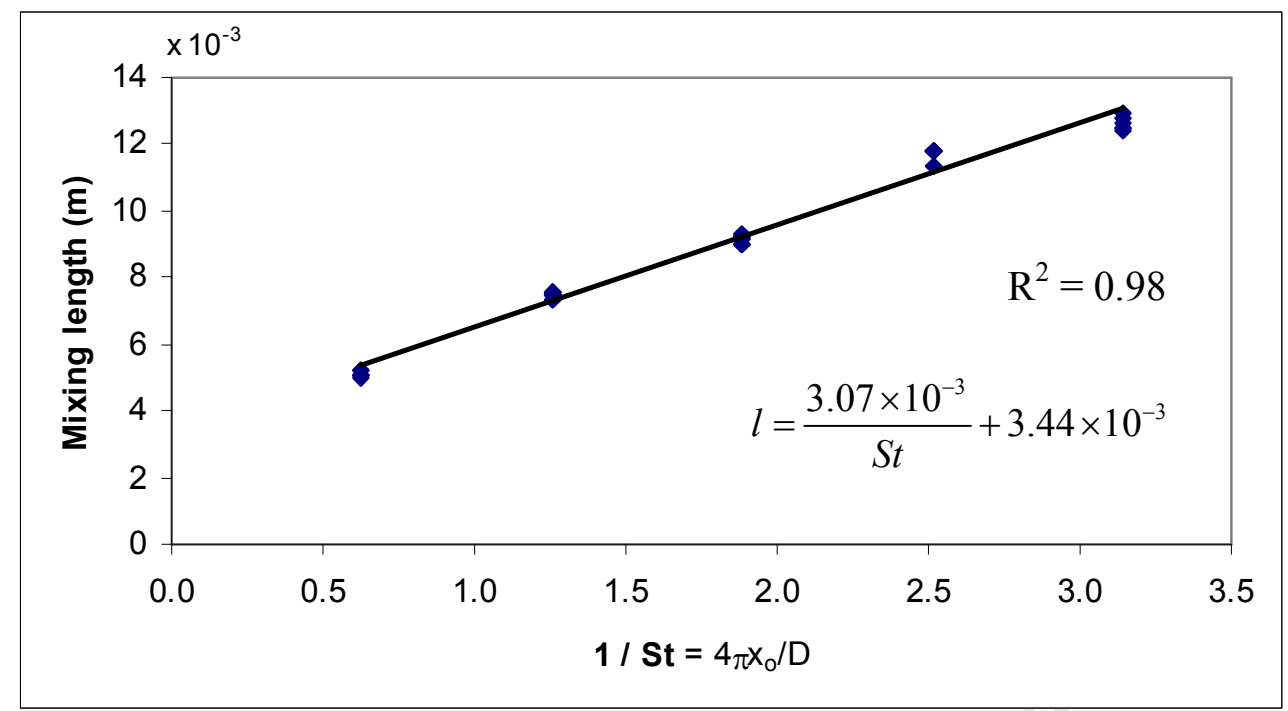

Figure 4.6 - Correlation Between Mixing Length and Strouhal Number

As was noted in section 2.2.2, recent studies by Fitch (2003) have indicated that the amplitude of oscillation controls the distance which eddies are propagated into the interbaffle cavities. The trend in Figure 4.6 shows strong correlation between the mixing length and the Strouhal number (dimensionless amplitude) indicating that turbulent eddies are indeed transported further at higher values of amplitude, regardless of the power dissipation in the fluid. The trend in Figure 4.6 therefore appears to be theoretically correct even though the mixing length was determined by fitting the data.

Interestingly, the values of mixing length obtained were found to be significantly lower than the column diameter $(100 \mathrm{~mm})$. These values were however within range of the mixing lengths reported by previous researchers. For example, Baird \& Stonestreet (1995) reported a mixing length of $7 \mathrm{~mm}$ for a $12 \mathrm{~mm}$ diameter OBC, and Baird et. al. (1996) reported a mixing length of $9 \mathrm{~mm}$ for a $150 \mathrm{~mm}$ diameter OBC. This suggests that the mixing length may in fact not be a strong function of the column diameter, as has been speculated by previous researchers.

Using the correlation in Figure 4.6 together with equation 4.2, one is able to predict the time-averaged power dissipation in the $\mathrm{OBC}$ to within $7 \%$ for ungassed operation. This 
provides a good indication that the results obtained using the Hafez \& Prochazka technique are valid in terms of the mean power dissipation obtained.

\subsubsection{Gassed Power Characteristics}

Power dissipation was also measured during each flotation test using the method outlined in Section 3.2.1. The results were then compared with the predictions of the correlation derived for ungassed conditions in order to evaluate the change in characteristics due to gas addition. The following section details the effect of gas addition on the measured power dissipation during both the quartz and nickel ore slimes flotation tests.

\subsubsection{Quartz Flotation}

For most conventional flotation systems, the power dissipation is thought to decrease with increasing gas hold-up. As will be detailed in Section 4.3.1, the gas hold-ups during quartz flotation were low compared with most flotation cells $(<5.1 \%)$. Under these conditions, gas addition was found to have little effect on the time-averaged power dissipation in the cell, as shown in the Figure 4.7: 


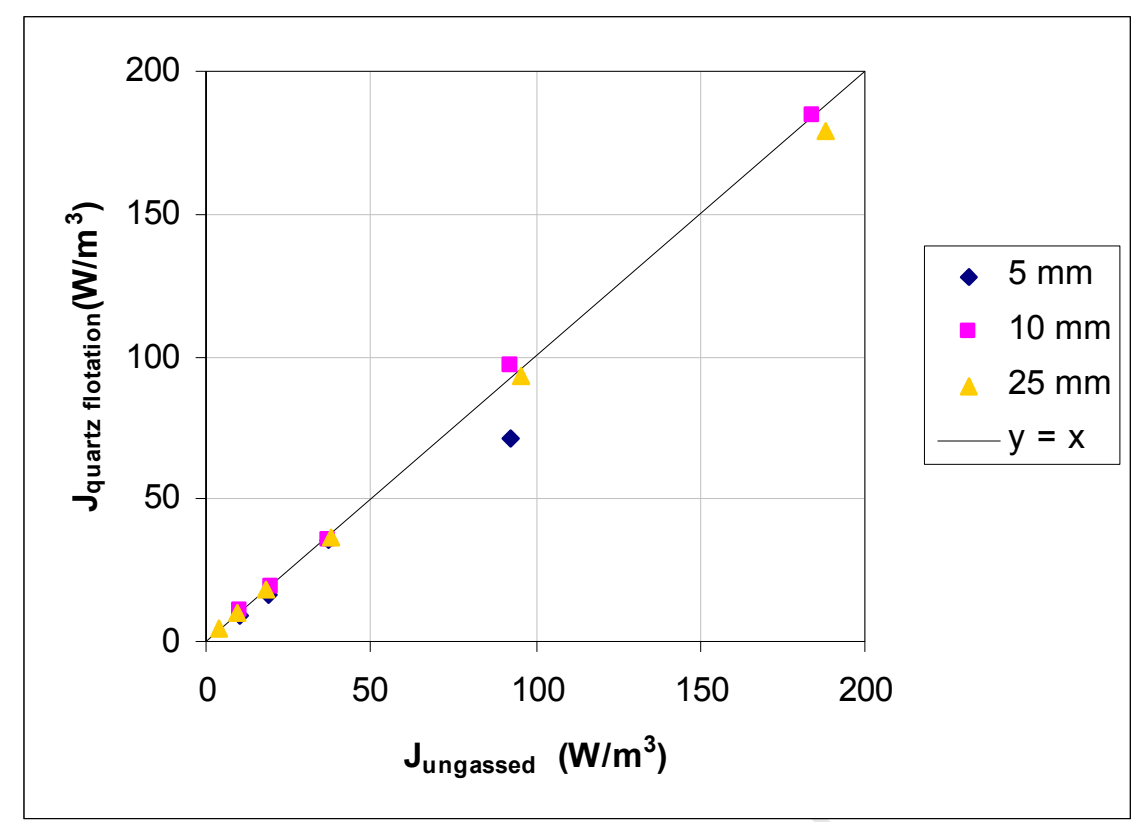

Figure 4.7 - Parity Chart Comparing Power Dissipation for Ungassed Conditions and the Power Input during Quartz Flotation Tests

Figure 4.7 illustrates the good agreement obtained between the ungassed power correlation's predictions and the measured power dissipation in the OBC. This is in agreement with the findings of Baird et. al. (1996), who found little effect of gas holdup on the power dissipation behaviour of a similar OBC for gas hold-ups up to $6.5 \%$.

\subsubsection{Nickel Ore Slimes Flotation}

When the OBC was trialled on nickel ore slimes, significantly higher gas hold-ups of between $21.8 \%$ and $35.4 \%$ were obtained. The resulting power behaviour started to deviate from the ungassed behaviour as illustrated in Figure 4.8: 


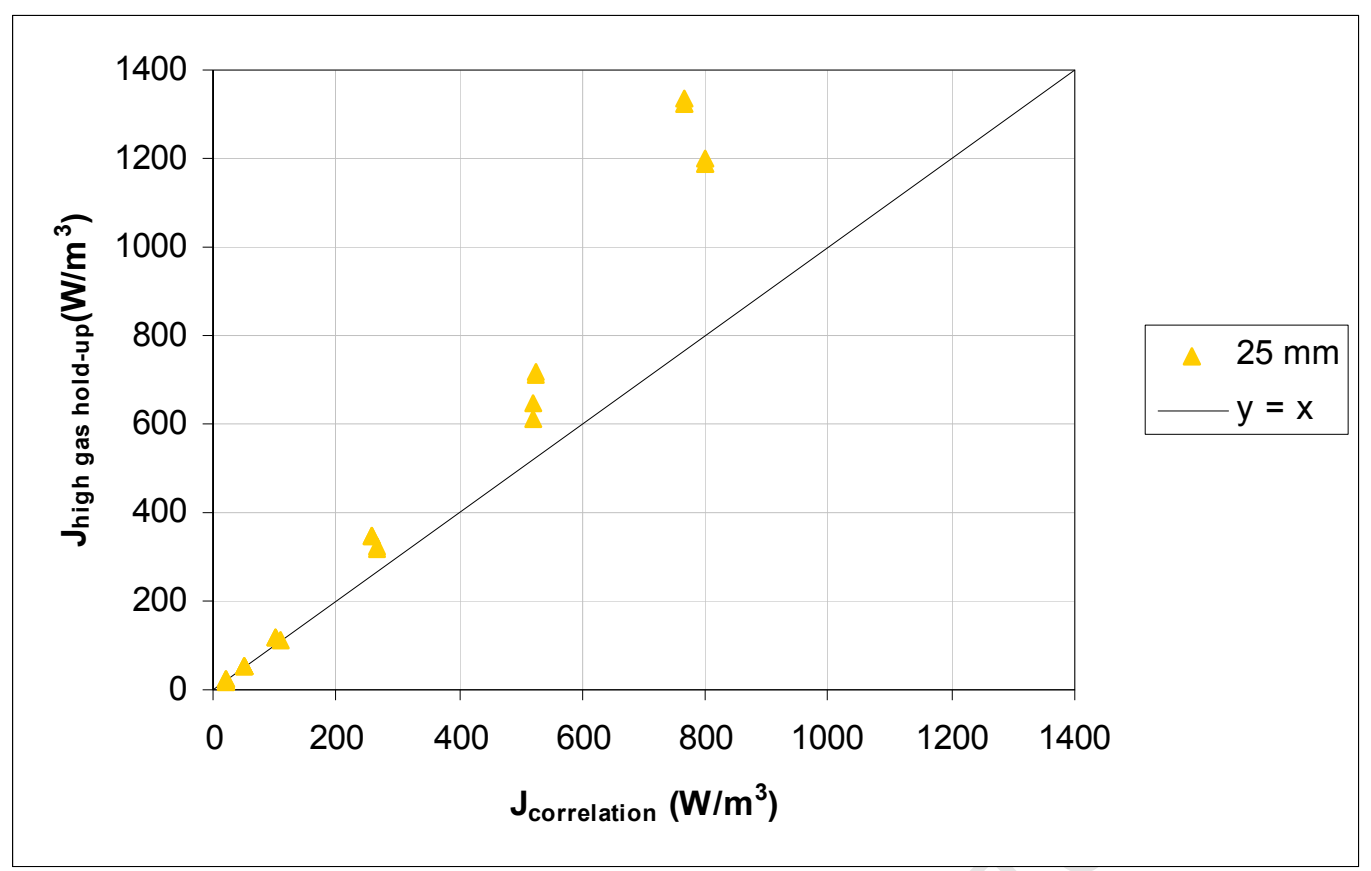

Figure 4.8 - Parity Chart between the Power Measured under Gassed Conditions and the Power Correlation for Ungassed Power for Gas Hold-Up between $21.8 \%$ and $35.4 \%$

As shown in Figure 4.8, the correlation for ungassed power dissipation greatly under estimates the power dissipation obtained under high gas hold-up conditions, particularly for power inputs of greater than $200 \mathrm{~W} / \mathrm{m}^{3}$. The reason for this behaviour is believed to be a result of an experimental error due to the compression of the gassed slurry under inertial forces.

As discussed in section 2.2.5, the overall pressure signal is thought to arise from an inertial and frictional component. The inertial component is modelled as proportional to the acceleration of the baffle cage and the frictional component is proportional to the cage velocity squared. This modelling technique therefore relies on the instantaneous transmission of the pressure wave from the baffles to the pressure transducer. This is a reasonable assumption in systems containing a single incompressible fluid, where the pressure waves travel unimpeded through the fluid at the speed of sound. In a gassed system however, the fluid environment is no longer incompressible and gas bubbles in the system are able to expand and contract as the pressure wave passes through them. It 
is hypothesised that this effect could have caused the error in the power calculation at high power input and gas hold-up.

This is illustrated in the Figure 4.9, where the measured pressure and the fitted pressure curve (c.f. Equation 3.2) are plotted as a function of the displacement of the plate stack. The time-averaged power dissipation in these diagrams is proportional to the area bounded by the curves (Baird \& Garstang, 1967):

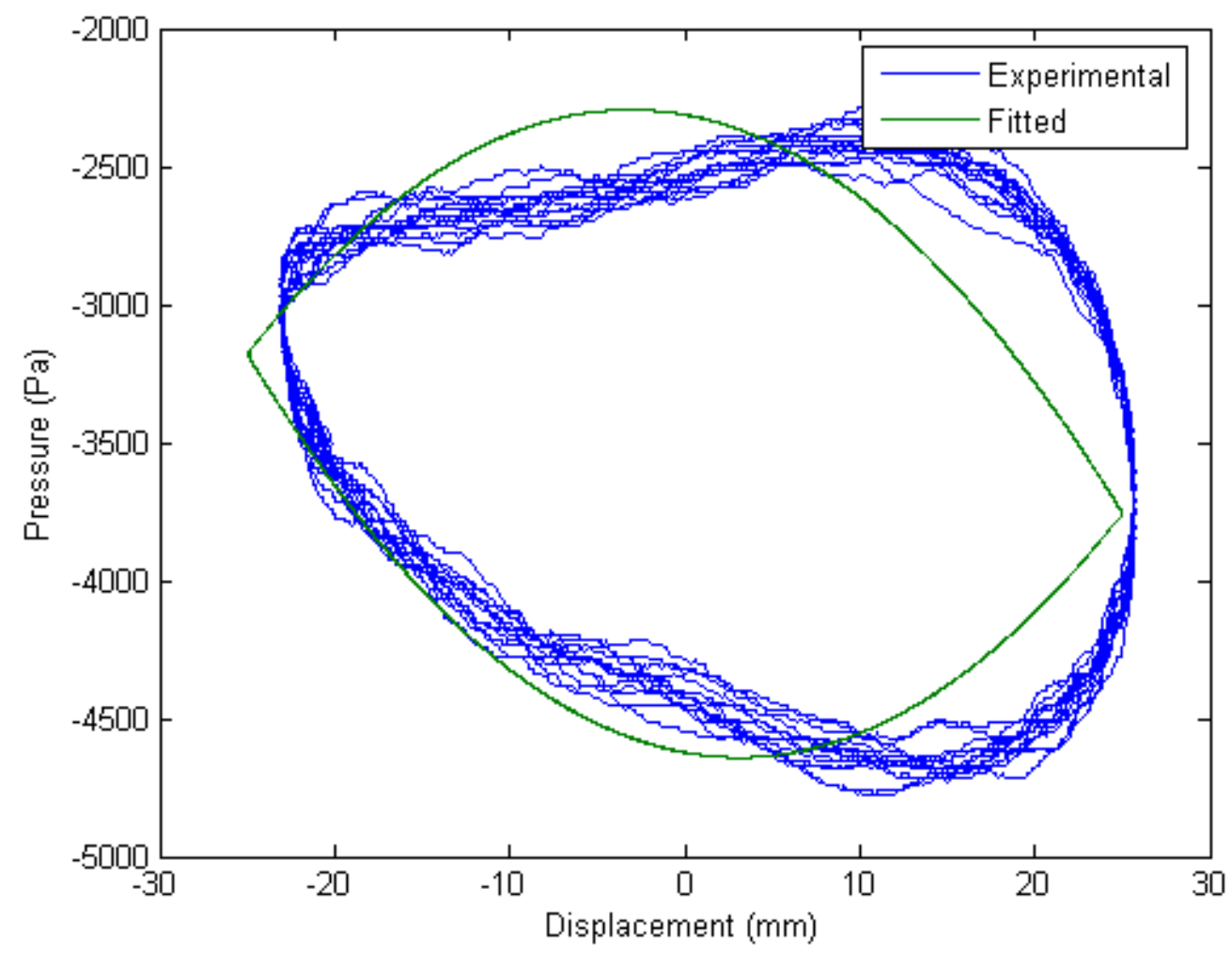

Figure 4.9 - Diagram Showing the Difference Between the Measured Pressure Signal and the Fitted Pressure Curve for the OBC Operating at $25 \mathrm{~mm}$ and $1.35 \mathrm{~Hz}$ with a Gas HoldUp of $30.0 \%$

Figure 4.9 shows that the pressure behaviour in the OBC under high gas hold-up conditions deviates significantly from modelled behaviour. Similar behaviour was noted by Baird et. al. (1996) who noted that under gassed conditions, significant deviations in the pressure signal occurred, particularly at the points of largest acceleration of the plate 
stack. It was speculated that this deviation may be due to an additional virtual mass of water moving with each bubble which may have affected the inertia term in the fitted pressure equation.

Although the shape of the curve was affected by gas addition for all flotation tests, the average power dissipation (proportional to the area bounded by the curve) remains approximately the same, particularly at low gas hold-ups. For the case of nickel ore slimes flotation however, the area bound by the curve increases slightly, indicating that additional energy is dissipated by the presence of bubbles in the system. For this reason, the time-averaged power dissipation of the OBC operating at high gas hold-ups was calculated from the measured pressure signal rather than the fitted signal as this signal is thought to be more representative of the actual power dissipation in the system. The mathematical description of the pressure waves in an OBC under high gas hold-up conditions is however clearly a complex problem which requires significant further research. The development of an improved power model for high gas hold-up systems is however beyond the scope of this project.

\subsection{The Effect of Power Dissipation on Bubble Size}

As was shown in Section 2.3, bubble size is thought to have an effect on all flotation sub-processes. Following the methodology of other experimental studies on power input (Ahmed \& Jameson, 1985, Deglon, 1998, Pyke et. al., 2003, Newell, 2006), it was desirable to obtain a constant bubble size distribution with increasing power dissipation. This would mean that changes in flotation kinetics could be attributed to changes in power dissipation rather than changes in gas phase characteristics. It was therefore important to quantify the effect of power dissipation on bubble size, since changes in bubble size may have an indirect effect on the flotation kinetics as a function of power dissipation.

Figure 4.10 shows the effect of power dissipation on the bubble size in the OBC. Bubble size is reported in terms of the arithmetic mean since this is the value best suited for inclusion in the fundamental flotation models. 


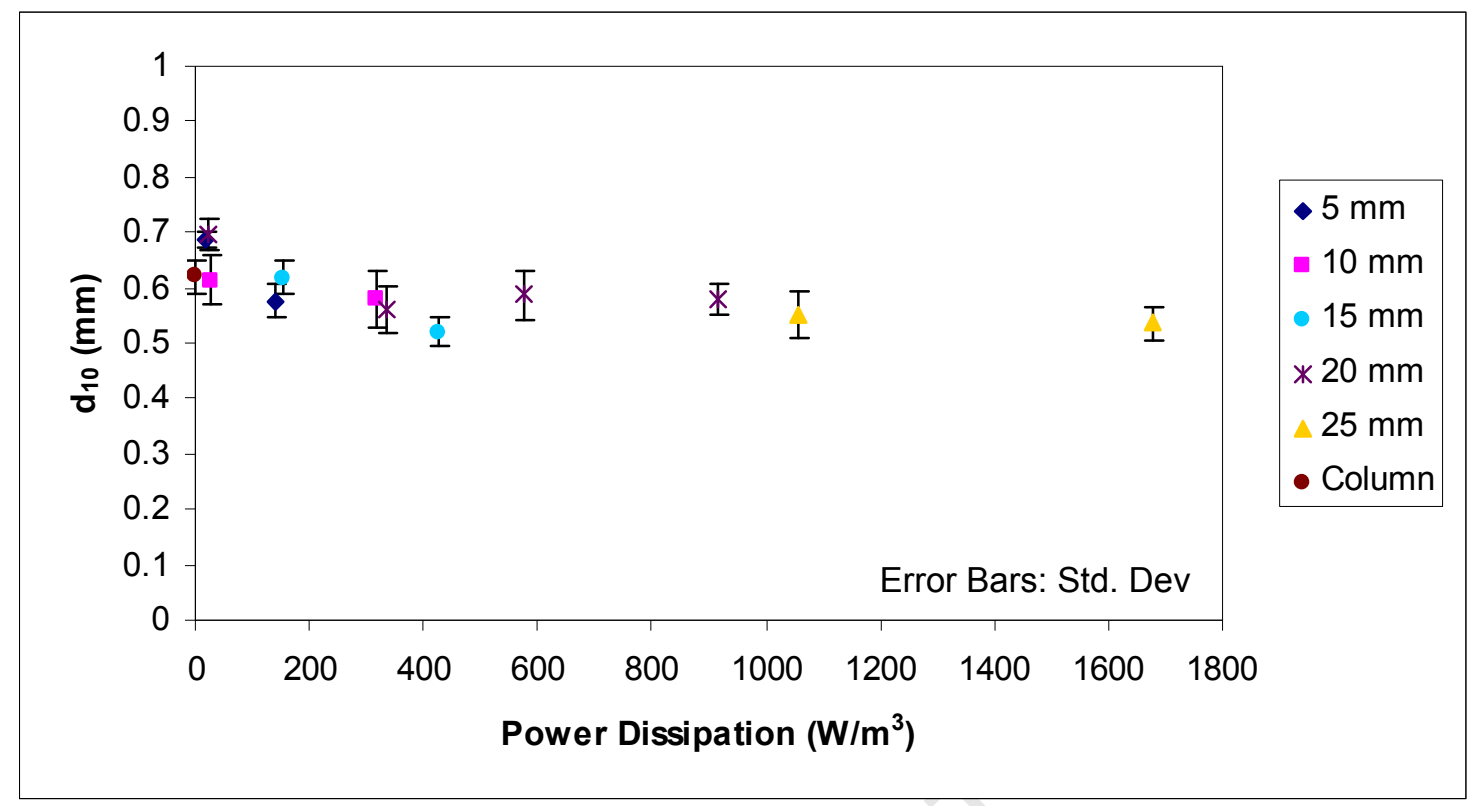

Figure 4.10 - The Effect of Power Dissipation on the Bubble Size in the OBC

Figure 4.10 shows that the mean bubble size in the $\mathrm{OBC}$ is largely unaffected by power dissipation even when power dissipation is varied by almost two orders of magnitude. Overall, the bubble size remains relatively constant with a mean of $0.60 \mathrm{~mm}$ and a standard deviation of $0.05 \mathrm{~mm}$. A slight increase in the bubble size was measured at the lower power inputs of 17 and $21 \mathrm{~W} / \mathrm{m}^{3}$, most likely due to a slight coalescence of bubbles behind the baffles. The lack of significant bubble breakage with increasing power dissipation is thought to be due a combination of the fine bubble size generated by the static mixer and the low power dissipations obtained in the agitated zone. This range of power dissipation is substantially lower than that found in the impeller zone of most conventional cells, which range from 5 to 200 times the average power dissipation in the cell (Schubert, 1999).

Bubble break-up under turbulence is often characterised by the critical Weber number, which is defined as the ratio of disruptive stresses to stabilising elastic stresses which are experienced by bubbles under turbulent flow conditions, viz.(Hinze, 1955) 
$W e_{c}=\frac{2 \cdot \rho \cdot \varepsilon^{2 / 3} \cdot d_{\max }^{5 / 3}}{\sigma}$

where $\mathrm{We}_{\mathrm{c}}$ is the critical Weber Number, usually between 0.9 and 4.7 (Parthasarathy et. al., 1991), $\sigma$ is the surface tension of the bubble in $\mathrm{N} / \mathrm{m}$ and $\mathrm{d}_{\max }$ is the maximum stable bubble size under the given conditions in metres. Assuming a surface tension of 0.07 $\mathrm{N} / \mathrm{m}$ and the minimum critical Weber number of 0.9 , equation 4.3 indicates that the maximum stable bubble size for the largest power dissipation $\left(1682 \mathrm{~W} / \mathrm{m}^{3}\right)$ is approximately $1.6 \mathrm{~mm}$. This is well beyond the range of bubble sizes measured and indicates that this range of power dissipations is unlikely to have had an effect on the bubble size. The lack of change in the bubble size with added power dissipation is therefore in line with theoretical expectations.

The slight increase in the bubble size measured at the lower power inputs of $17 \mathrm{~W} / \mathrm{m}^{3}$ and $28 \mathrm{~W} / \mathrm{m}^{3}$ was most likely due to coalescence of larger bubbles as they rise through the agitated zone. Under these settings the baffle cage velocity was low compared with the bubble rise velocity, resulting in some of the larger bubbles collecting behind the baffles plates at some points in the cycle. This resulted in an increased probability of coalescence of bubbles at each baffle, resulting in a marginally larger bubble size being measured at the top of the plate stack. Preliminary tests suggested that the frother dosage was greater than the critical coalescence concentration; however given the close congregation of bubbles behind the baffles, it is believed that some coalescence may still have occurred. These measurements therefore represent the maximum bubble size distribution which would be obtained in the agitated zone, after multiple stages of coalescence. It is therefore believed that this increase is more an artefact of the position of bubble size measurement than a true reflection of the mean bubble size in the agitated zone. If significant, such an increase in bubble size would result in a decrease in the flotation rate from a standard column at low power dissipation. The significance of this increase will therefore be assessed when examining the flotation data in Chapter 5. 


\subsection{The Effect of Power Dissipation on Gas Hold-up}

Gas hold-up measurements were taken as part of the standard operating procedure during flotation tests. This was because the turbulent fundamental models of flotation suggest that a linear relationship exists between the flotation rate and the number of bubbles per unit volume (c.f. equation 2.32). Since the mean number of bubbles per unit volume is characterised by the gas hold-up, one would expect the flotation rate to vary in direct proportion to this quantity. Other semi-empirical models of flotation however indicated that the flotation rate is in fact dependent on the bubble surface area flux through the cell and is therefore independent of gas hold-up (Gorain et. al., 1997, Deglon, 2002, Harris et. al., 2002). The comparison of trends in gas hold-up with trends in flotation rate therefore provides a useful means of assessing this apparent paradox between flux and volumetric based models. The gas phase characteristics of both the quartz and nickel ore slimes systems are discussed separately in the Sections 4.3.1 and 4.3.2.

\subsubsection{Quartz Flotation}

Quartz flotation tests were conducted at two different superficial gas velocities of 0.12 $\mathrm{cm} / \mathrm{s}$ and $0.21 \mathrm{~cm} / \mathrm{s}$, corresponding to the moderately hydrophobic and weakly hydrophobic flotation conditions respectively (c.f. Table 3.3). The relationship between power dissipation and gas hold-up for these tests is shown in Figure 4.11: 


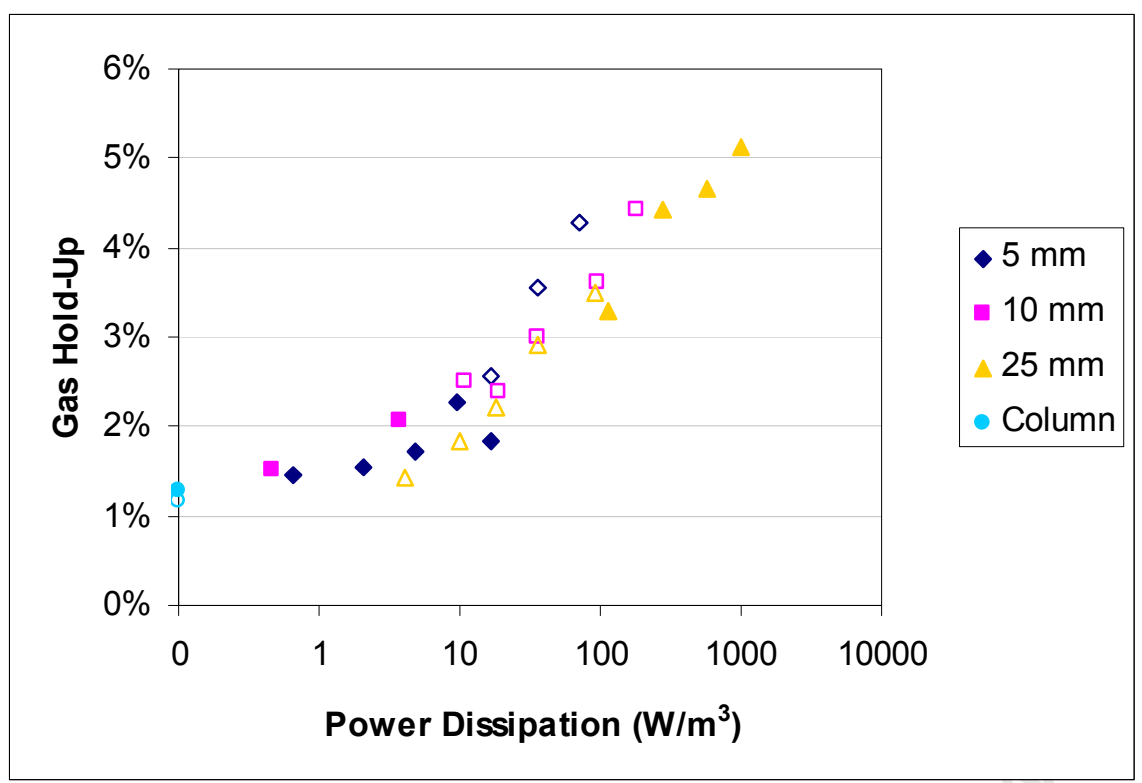

Figure 4.11 - The Relationship between Power Dissipation \& Gas Hold-up during Quartz Flotation Tests. (Open points denote $J_{g}=0.12 \mathrm{~cm} / \mathrm{s}$, Closed points denote $J_{g}=0.21 \mathrm{~cm} / \mathrm{s}$ )

Figure 4.11 shows that the gas hold-up in the $\mathrm{OBC}$ increases with increasing power dissipation. This increase is most likely due to the reversing flow action of the oscillation mechanism rather than a decrease in bubble size, since the bubble size was found to remain relatively constant over the range of power dissipations tested (c.f. Figure 4.10). This is in agreement with the results of Oliveira et. al. (2003) who reported that increased oscillation led to longer bubble residence times in the cell due to increased reversing flow under these conditions.

Overall the gas hold-ups in the cell were low for all settings (less than $5.1 \%$ ), due to the low $\mathrm{J}_{\mathrm{g}}$ used during test work. The relative change in gas hold-up over these conditions was however very large. For the weakly hydrophobic conditions tested, a 4.2 fold increase in gas hold-up was obtained from $1.3 \%$ to $5.1 \%$ and for the moderately hydrophobic conditions a 3.7 fold increase in gas hold-up was obtained from $1.2 \%$ to $4.4 \%$. This ability of the $\mathrm{OBC}$ to vary the gas hold-up over such a wide range independently of the bubble size provides a useful opportunity to test the effect of the number of bubbles per unit volume on flotation kinetics. Such an investigation would be 
considerably more difficult in a stirred tank environment where no reversing component of flow is present.

According to the models detailed in section 2.3, gas hold-up affects the rate constant by increasing the number of bubbles per unit volume in the cell. If the bubble size is assumed to be constant, the gas hold-up is directly proportional to the number of bubbles per unit volume in the system, which is in turn directly proportional to the flotation rate constant (c.f. Equation 2.32). Thus a four fold increase in gas hold-up would theoretically result in a four fold increase in the flotation rate constant. The validity of this relationship will be re-examined in Section 5.5.

\subsubsection{Nickel Ore Slimes Flotation}

Figure 4.12 shows the effect of the oscillation on the gas hold-up obtained during the nickel ore slimes flotation tests at both high and low percent solids. These tests were performed at a constant $\mathrm{J}_{\mathrm{g}}$ of $0.64 \mathrm{~cm} / \mathrm{s}$.

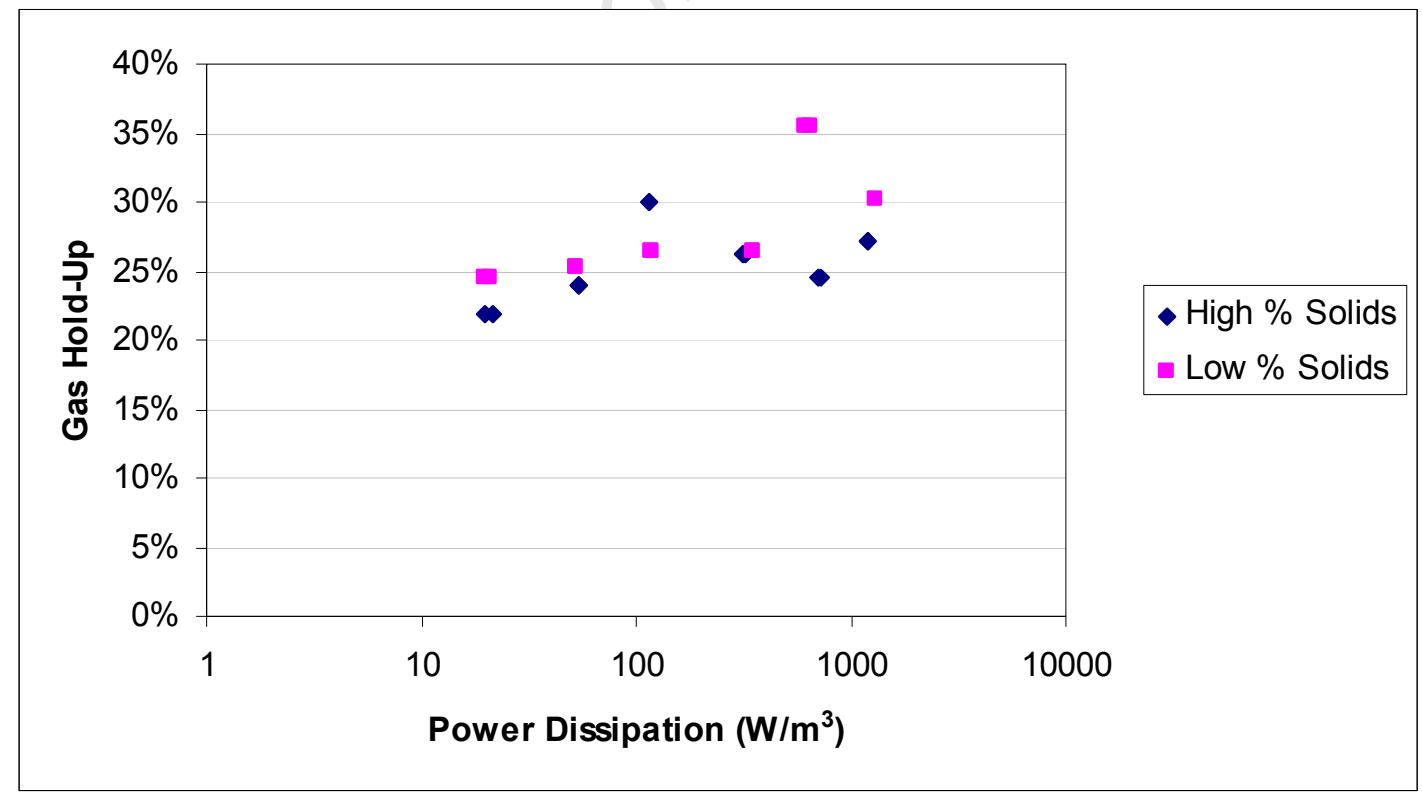

Figure 4.12 - The Effect of Power Dissipation on Gas Hold-up during Nickel Ore Slimes Flotation Tests. $\left(\mathrm{J}_{\mathrm{g}}=0.64 \mathrm{~cm} / \mathrm{s}\right)$ 
Once again the gas hold-up was found to generally increase with increasing power dissipation, although considerably more scatter is present in the data. Overall, the gas hold-up is significantly higher than that obtained during quartz flotation due to a combination of the increase in superficial gas velocity, and the low bubble rise velocity due to the highly viscous nature of the slurry. This reduced rise velocity was highly pronounced and, in some cases, the bubbles were in fact entrained into the tails of the cell. This complex behaviour resulted in high overall gas hold-ups and may have created additional complexities in the flotation rate data obtained.

Overall gas hold-ups varied between $21.8 \%$ and $35.4 \%$ for the range of settings tested. This corresponds to an increase of $62 \%$ in gas hold-up, which would correspond to a 62 $\%$ increase in the flotation rate constant assuming the predictions of the fundamental models are correct.

\subsection{The Effect of Power Dissipation on Mixing Characteristics}

The effect of power dissipation on the mixing characteristics was assessed in water-gas operation at the same set of conditions as the moderately hydrophobic quartz flotation tests (i.e. air rate $=0.55 \mathrm{l} / \mathrm{min}$; recycle rate $=6 \mathrm{l} / \mathrm{min}$; frother dosage $=10 \mathrm{ppm}$ ). The results are shown in Figure 4.13. 


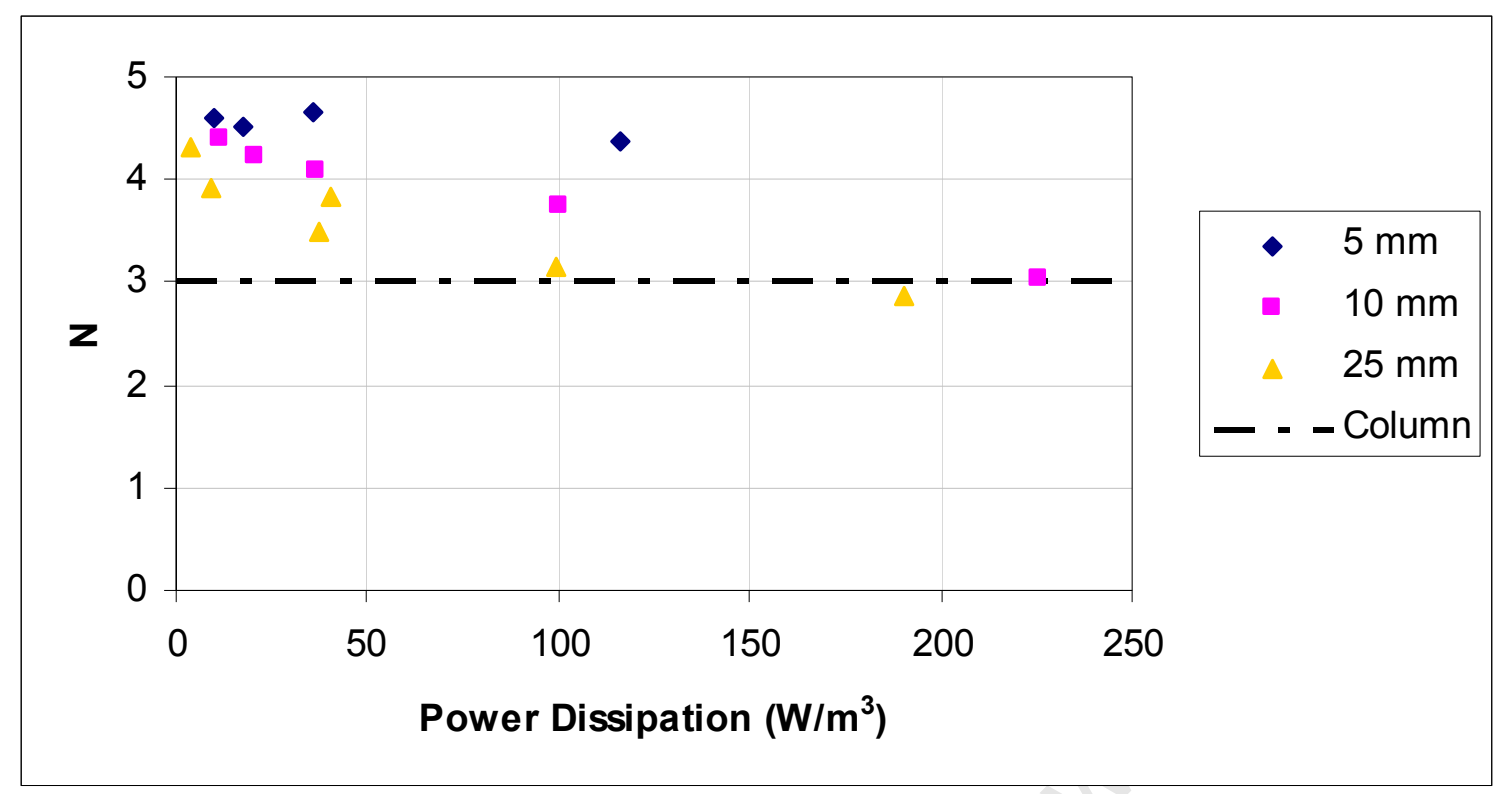

Figure 4.13 - The Effect of Power Input on the Mixing Characteristics in the OBC relative to a Standard Column

Figure 4.13 shows that the addition of oscillation produces a marked increase in the number of tanks-in-series, which increases from 3.0 in a standard column to 4.6 at a power dissipation of $10.1 \mathrm{~W} / \mathrm{m}^{3}$. These results indicate that the $\mathrm{OBC}$ is able to produce improved plug flow conditions relative to a standard flotation column, particularly at low frequencies and amplitudes. This suggests that the $\mathrm{OBC}$ is an inherently more efficient 'reactor-type', and is consistent with the OBC's application in many chemical reaction applications (Fabiyi \& Skelton, 1999, Stonestreet \& Harvey, 2002, Skelton et. al., 2005).

The number of tanks-in-series slowly declines from a maximum of 4.6 as the power dissipation is increased, with a greater decrease being observed at larger amplitudes of oscillation. This is consistent with the findings of Fitch (2003), who noted that greater eddy propagation was obtained at higher amplitudes. At these settings, eddies are transported further into the inter-baffle cavities, resulting in increased back-mixing.

To test the significance of these mixing characteristics on the overall kinetics, let us consider the effect of the number of tanks-in-series on the overall recovery in a flotation 
system. Using the tanks-in-series-model, the recovery can be described by the following equation (Levenspiel, 1962):

$R=1-\frac{1}{(1+k \cdot \tau / N)^{N}} \quad$ (for $\mathrm{N}$ tanks in series)

where $\tau$ is the residence time of the slurry, $\mathrm{k}$ is the rate constant excluding mixing effects and $\mathrm{N}$ is the number of tanks-in-series. Assuming a residence time of $2.7 \mathrm{~min}$, (the average residence time during quartz flotation), the number of tanks-in-series has the following effect on the recovery:

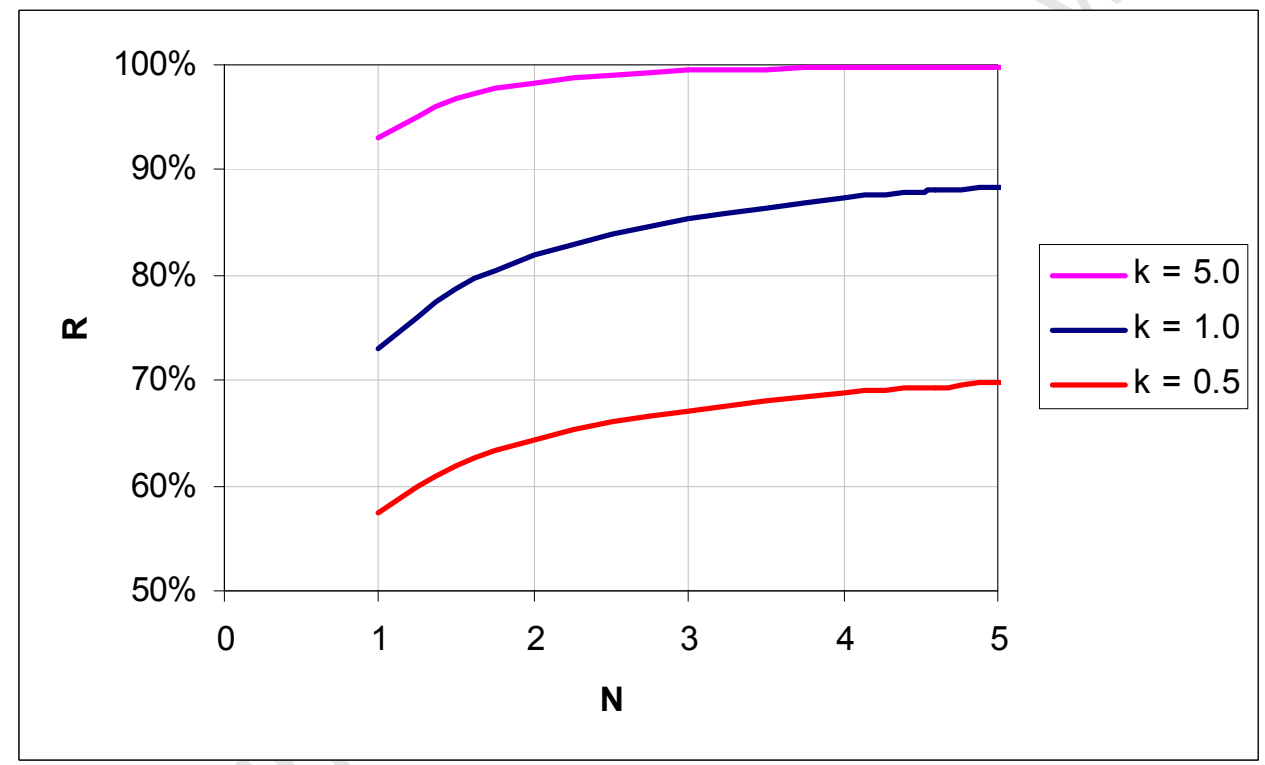

Figure 4.14 - The Effect of the Number of Tanks-in-Series on the Recovery of a Cell with a Residence Time of 2.7 min for Different Values of Rate Constant

As shown in Figure 4.14, the recovery increases with $\mathrm{N}$ at a decreasing rate. The magnitude of this increase is dependent on the initial recovery in the cell. For $\mathrm{k}=0.5$ $\min ^{-1}$, the recovery increases by $2.2 \%$, from $67.1 \%$ to $69.4 \%$ as $\mathrm{N}$ increases from 3.0 to 4.6 . However, for $\mathrm{k}=5.0 \mathrm{~min}^{-1}$, the recovery increases by only $0.4 \%$, from $99.4 \%$ to $99.8 \%$ since the recovery is already close to $100 \%$. The significance of these increases in recovery in terms of the overall rate constant can be determined by calculating the 
overall rate constant that would be obtained for each recovery in Figure 4.14 assuming perfect back mixing (i.e. $\mathrm{N}=1$ ). This is given by the following well known equation for the overall flotation rate constant:

$k_{\text {overall }}=\frac{1}{\tau} \cdot \frac{R}{1-R}$

(single CSTR)

If one then calculates the overall rate constants from the recoveries in Figure 4.14, one obtains the following relationship between the overall rate constant and the number of tanks-in-series:

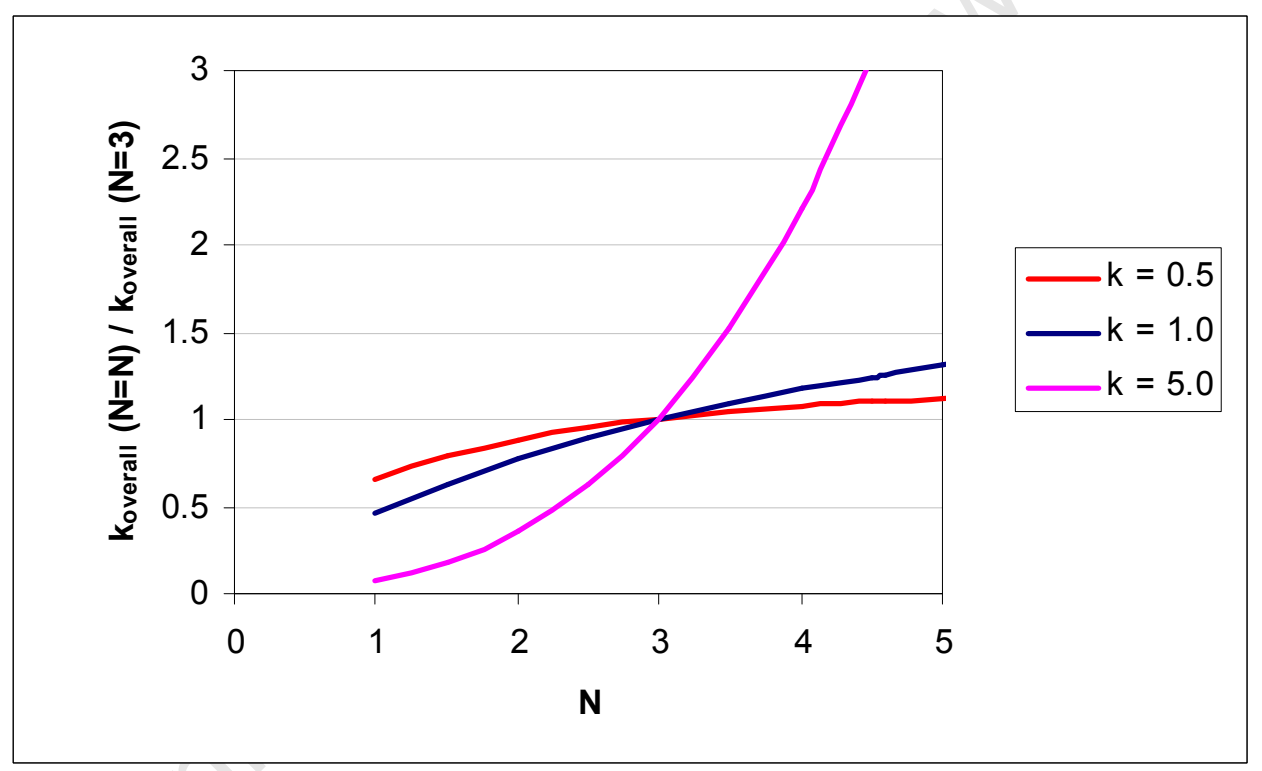

Figure 4.15 - The Effect of the Number of Tanks-in-Series on the Overall Rate Constant Normalised Relative to the Rate Constant obtained at $\mathbf{N}=3$

Figure 4.15 shows that the sensitivity of the overall rate constant to changes in $\mathrm{N}$ is a strong function of the overall recovery in the cell. When the overall recovery in the cell is less than $70 \%$ (i.e. $\mathrm{k}=0.5 \mathrm{~min}^{-1}$ ), the mixing characteristics of the system do not strongly affect the overall rate constant which is calculated. Under these conditions, the calculation of the overall rate constant neglecting mixing effects would only produce an $11 \%$ error if $\mathrm{N}$ was increased from 3.0 to 4.6. However, if the overall recovery in the 
system is large (i.e. greater than $90 \%$ ), the effect of mixing on the overall rate constant becomes highly significant and produces an almost $300 \%$ increase in the calculated rate constant.

The results therefore indicate that the observed change in $\mathrm{N}$ from 3.0 to 4.6 , may have a significant effect on the overall rate constant calculated for moderate to high flotation recoveries $(\mathrm{R}>70 \%)$. This information should be carefully considered when interpreting flotation data. For systems which exhibit a high recovery, a tanks-in-series model should be used to account for the effect of macro-mixing on the overall rate constant. This would help to decouple the mixing effects from the effect of power dissipation on the overall rate obtained. For systems with low recoveries however, the effect of mixing on the overall rate constant becomes negligible and the tanks-in-series model is therefore not necessary.

It should be noted however that when applying the tanks-in-series model to flotation systems, it is assumed that particles in the cell perfectly follow the fluid and therefore have the same residence time distribution. This assumption seems valid for particles with Stokes numbers less than 1 (c.f. Equation 2.35). For quartz particles in a system with a mean energy dissipation rate of $1000 \mathrm{~W} / \mathrm{m}^{3}$, this equates to particles of less than 75 microns (c.f. Figure 2.16). For larger particle sizes, some error may become apparent due to possible differences between the particle and fluid residence times.

\subsection{Summary of Findings and Implications}

This chapter has detailed both the characterisation of the power dissipation in the OBC and its effect on flotation variables such as bubble size, gas hold-up and mixing. The power characteristics of the $\mathrm{OBC}$ were first evaluated in water-only operation and the observed behaviour was found to be in good agreement with theoretical predictions. The measured power dissipation during quartz flotation correlated well with ungassed power dissipation predictions since the gas hold-up during the flotation tests was low. For nickel slimes ore flotation however, the pressure signal could not be adequately fitted using existing models from the literature. The time-averaged power dissipations during 
these tests were therefore calculated using the raw pressure data, rather than the fitted pressure curves.

The bubble size in the column was evaluated over a wide range of power dissipations and was found to remain relatively constant with a mean of $0.60 \mathrm{~mm}$ and a standard deviation of $0.05 \mathrm{~mm}$. A slight increase in the bubble size was noted at low power dissipation where a build up of bubbles behind the baffles at some points in the cycle may have resulted in coalescence of bubbles behind each baffle. This increase in bubble size would theoretically result in a decrease in the flotation rate relative to a standard column at low power dissipation conditions. The sensitivity of the flotation rate to this apparent change in bubble size will be assessed in Chapter 5.

The gas hold-up characteristics of the $\mathrm{OBC}$ were also evaluated as a function of power dissipation during flotation tests. Results indicate that the $\mathrm{OBC}$ is able to substantially increase the gas hold-up in the system independently of the bubble size. This is due to the reversing flow action of the $\mathrm{OBC}$ obtained at high frequencies and amplitudes. Oscillation produced a 4.2 fold increase in gas hold-up during the weakly hydrophobic quartz flotation tests, a 3.7 fold increase during the moderately hydrophobic quartz flotation tests and a $67 \%$ increase during the nickel slimes flotation tests.

The fundamental models of flotation predict a linear relationship between the flotation rate and the number of bubbles per unit volume in the cell. If this prediction is indeed correct one would anticipate a one-to-one increase in the flotation rate with increasing gas hold-up. These models therefore predict substantial increases in the rate constant.

The OBC's ability to vary the gas hold-up independently of large changes in bubble size creates an interesting opportunity to evaluate this modelling premise. While fundamental flotation models are derived on a volumetric basis, many other semiempirical models of flotation have indicated that the flotation rate is in fact dependent on the bubble surface area flux through the cell (Gorain et al., 1997, Deglon, 2002, Harris et al., 2002). In a conventional stirred system, the modelling approaches are largely equivalent since an increase in bubble surface area flux results in an increase in 
gas hold-up. In the OBC however, the gas hold-up can be varied over a wide range with comparatively little effect on the bubble size. This therefore allows the relationship between gas hold-up and flotation rate to be more closely examined and constitutes a major advantage of applying the $\mathrm{OBC}$ as a research tool.

The mixing characteristics of the $\mathrm{OBC}$ were also evaluated under the same conditions as those used in the moderately hydrophobic quartz flotation tests. Results indicated that the $\mathrm{OBC}$ is able to produce improved plug flow conditions relative to a standard flotation column, particularly at low frequencies and amplitudes. As the frequency and amplitude are increased, the mixing conditions start to more closely approximate those of the standard column. A sensitivity analysis using the tanks-in-series model showed that for low recoveries $(<70 \%)$ the effect of mixing on the rate constant is not significant; however as the recovery increases, the effect of mixing on the rate constant becomes highly significant and should be taken into account.

The following chapters analyse the flotation performance of the $\mathrm{OBC}$ in treating quartz and nickel ore slimes, with specific emphasis on the effect of power dissipation on the kinetics and the extent to which the results are in agreement with flotation model predictions. 


\section{Chapter 5}

\section{Quartz Flotation}

In order to assess the hypotheses of this thesis, the flotation response of the OBC was evaluated as a function of power dissipation using a quartz-amine system at three different amplitudes and at two different collector dosages. The work was performed in two stages, with preliminary tests being conducted over a wide range of power dissipations, under the same particle hydrophobicity conditions as Deglon (1998). The results of these tests were then used to inform the development of a second investigation which was conducted over a more focussed range of power dissipations. This set of tests was also conducted at a higher collector dosage in order to investigate the change in flotation response with increased hydrophobicity. Section 5.1 details the results obtained from the initial pre-characterisation of the flotation response, carried out under weakly hydrophobic conditions (c.f. Table 3.3), before Section 5.2 details the flotation response obtained under moderately hydrophobic conditions.

\subsection{Flotation Response under Weakly Hydrophobic Conditions}

The following section details the flotation response of quartz under weakly hydrophobic conditions. The objective of this work was to pre-characterise the flotation response of quartz as a function of power dissipation and benchmark the study against the results of Deglon (1998). 


\subsubsection{Particle Size vs. Recovery}

The relationship between particle size and flotation recovery is shown in Figure 5.1:

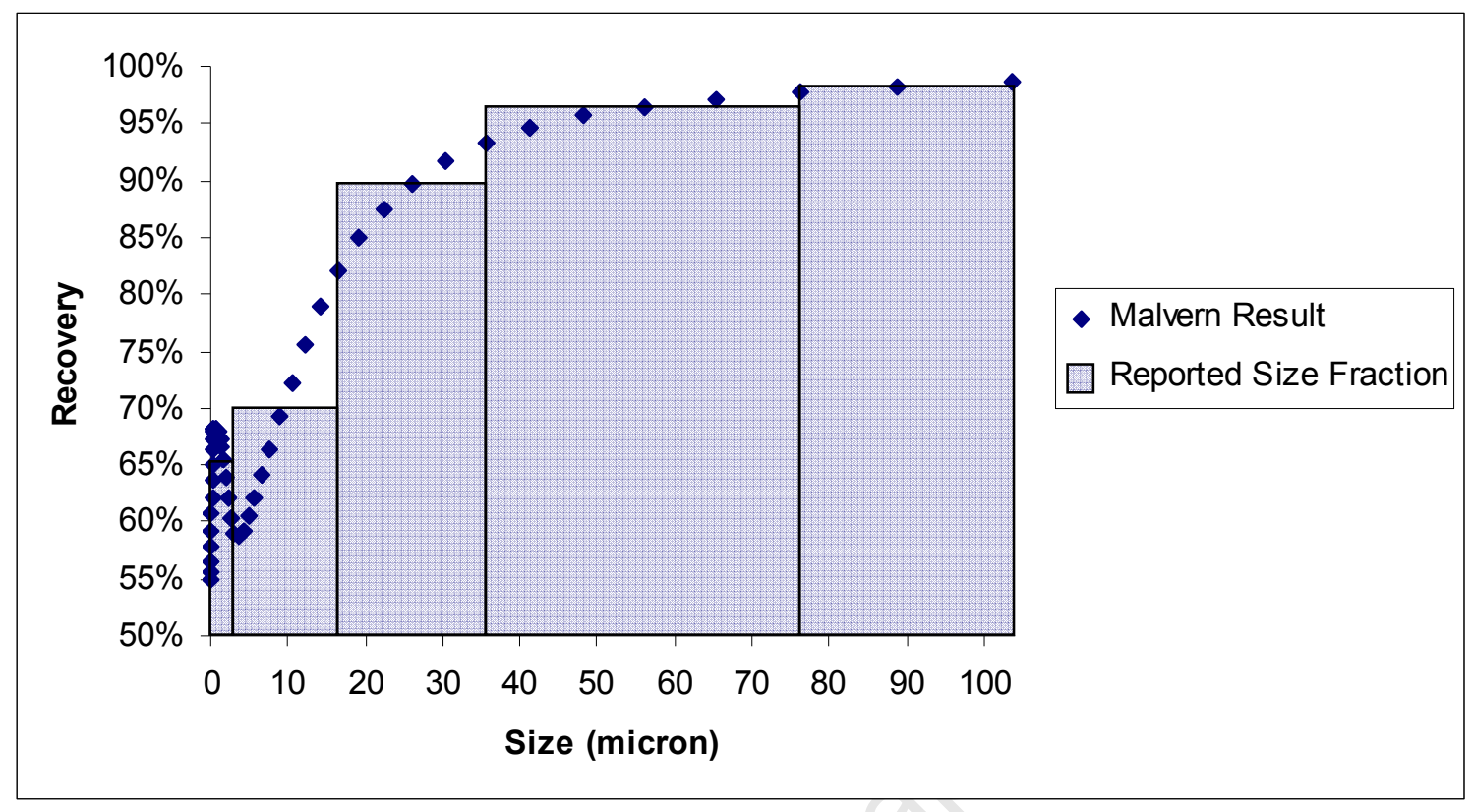

Figure 5.1 - The Relationship between Particle Size and Recovery for the OBC Operating as a Standard Column Cell under Weakly Hydrophobic Conditions (c.f. Table 3.3)

Figure 5.1 shows the relationship between particle size and recovery for the OBC operating as a standard column. As expected, the recovery increases as particle size increases from 3 microns to 104 microns. This is in line with flotation theory and similar results have been reported by numerous other researchers (Ahmed \& Jameson, 1985, Deglon, 1998, Pyke, 2004, Newell, 2006). The above figure is typical of the flotation response obtained for quartz as a function of particle size, with the curve being marginally shifted up or down depending on the size-by-size recovery obtained as a function of operating conditions.

Interesting to note is the high flotation recovery obtained for particle sizes greater than 36 micron. This is because particles of this size were in fact highly fast floating relative to the residence time in the system, most likely due to the fine bubble size distribution obtained using the static mixer. This is illustrated by comparison of the results in Figure 
5.1 with the results obtained in a standard column cell of similar dimensions using a standard rubber sparger (Breytenbach, 1995) (c.f. Figure 2.5 on page 20). It appears that the incorporation of the static mixer into the column design was able to produce substantial improvements in quartz recovery for all particle size fractions even at the low bubble surface area flux of $11.0 \mathrm{~s}^{-1}$.

The size-by-size recoveries in Figure 5.1 were subsequently grouped into 5 different size fractions based on their similar behaviour with respect to power input. These were the sub 3 micron, 3 -17 micron, 17 - 36 micron, 36 - 76 micron and 76 - 104 micron size fractions which are shown in Figure 5.1. The following section details the behaviour of the sub 3 micron size fraction. Thereafter, Section 5.1.3 details the change in flotation response of the other size fractions as a function of power dissipation.

\subsubsection{Behaviour of the Sub 3 Micron Size Fraction}

For particles sizes below 3 microns, there is an increase in the flotation recovery of ultra-fine material, as shown in Figure 5.1. This is behaviour is unexpected and has not been reported by previous researchers who usually quote the rate constant from 3 microns upwards. Recently some researchers have demonstrated that the recovery of such ultra-fine particles is controlled by a combination of Brownian motion and electric double layer effects (Nguyen et. al., 2006) with the collection efficiency increasing as particle size increases above $100 \mathrm{~nm}$ but decreasing as particle inertia becomes more significant. The trends obtained with particle size below 3 micron would seem to support this hypothesis.

An added complication to the analysis however is that one cannot discount the possibility of agglomeration of these particles with larger particles in feed during the feed preparation. Particles of this size are likely to be dominated by surface force effects. Metal cations, such as iron which may have been deposited during milling, could have rendered parts of their surface positively charged. This would lead to agglomeration of these particles onto the surfaces of other negatively charged quartz particles causing them to be recovered with the coarser size fractions. This effect, 
known as carrier flotation, has also been demonstrated in the literature (Valderrama \& Rubio, 1999). Larger particles would be less susceptible to this effect since their surface charge per unit mass is significantly less.

Thus, while the flotation behaviour of this ultrafine fraction could give an indication of possible Brownian motion and electric double layer effects as shown by Nguyen et. al. (2006), the behaviour of this size fraction cannot be explicitly addressed without significant speculation. The investigation of the flotation behaviour of this size fraction is therefore believed to require a more focussed study which can quantify the magnitude of the surface forces between the particles and bubbles and tightly control the temperature of the flotation environment. The flotation of this finest size fraction is however of little practical interest to most flotation applications and is therefore of limited interest to the current study. Further investigation of this size fraction is therefore defined as a task for future work.

\subsubsection{The Effect of Power Dissipation on Flotation Recovery}

Figures 5.2, 5.3, 5.4 and 5.5 show the flotation response of weakly hydrophobic quartz as a function of power dissipation. 


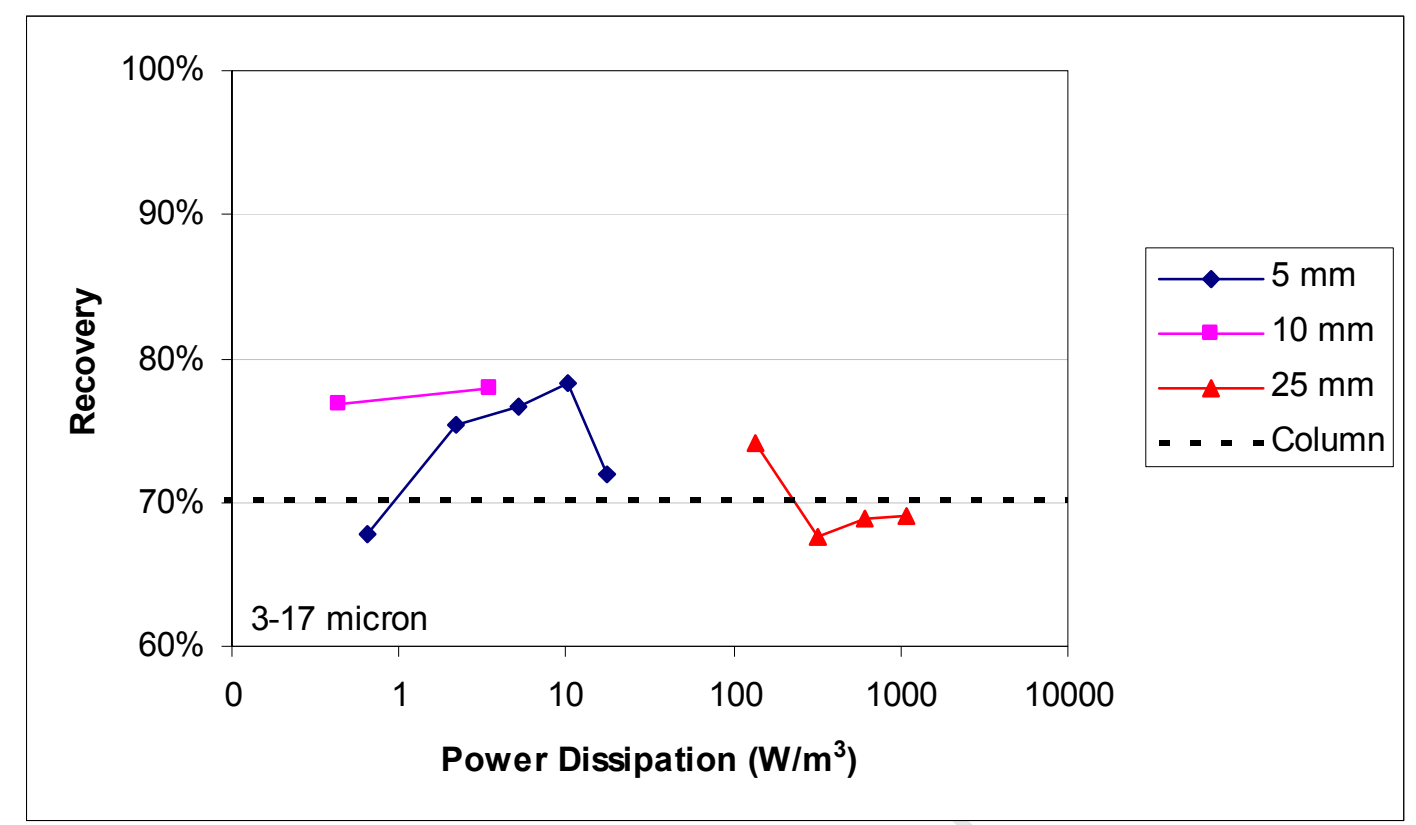

Figure 5.2 - The Effect of Power Dissipation on the Recovery of Weakly Hydrophobic 3 - 17 micron Quartz Particles

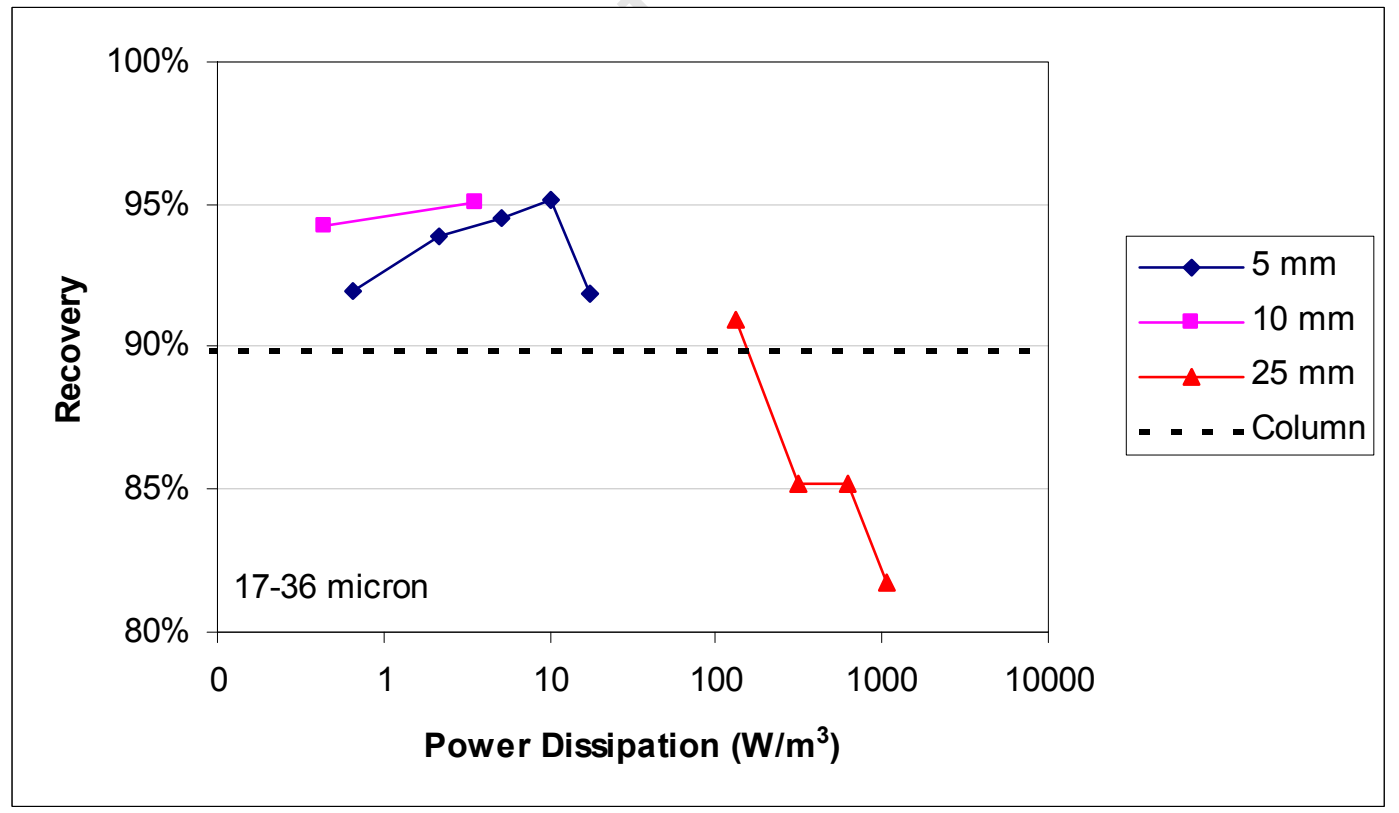

Figure 5.3 - The Effect of Power Dissipation on the Recovery of Weakly Hydrophobic 17 - 36 micron Quartz Particles 


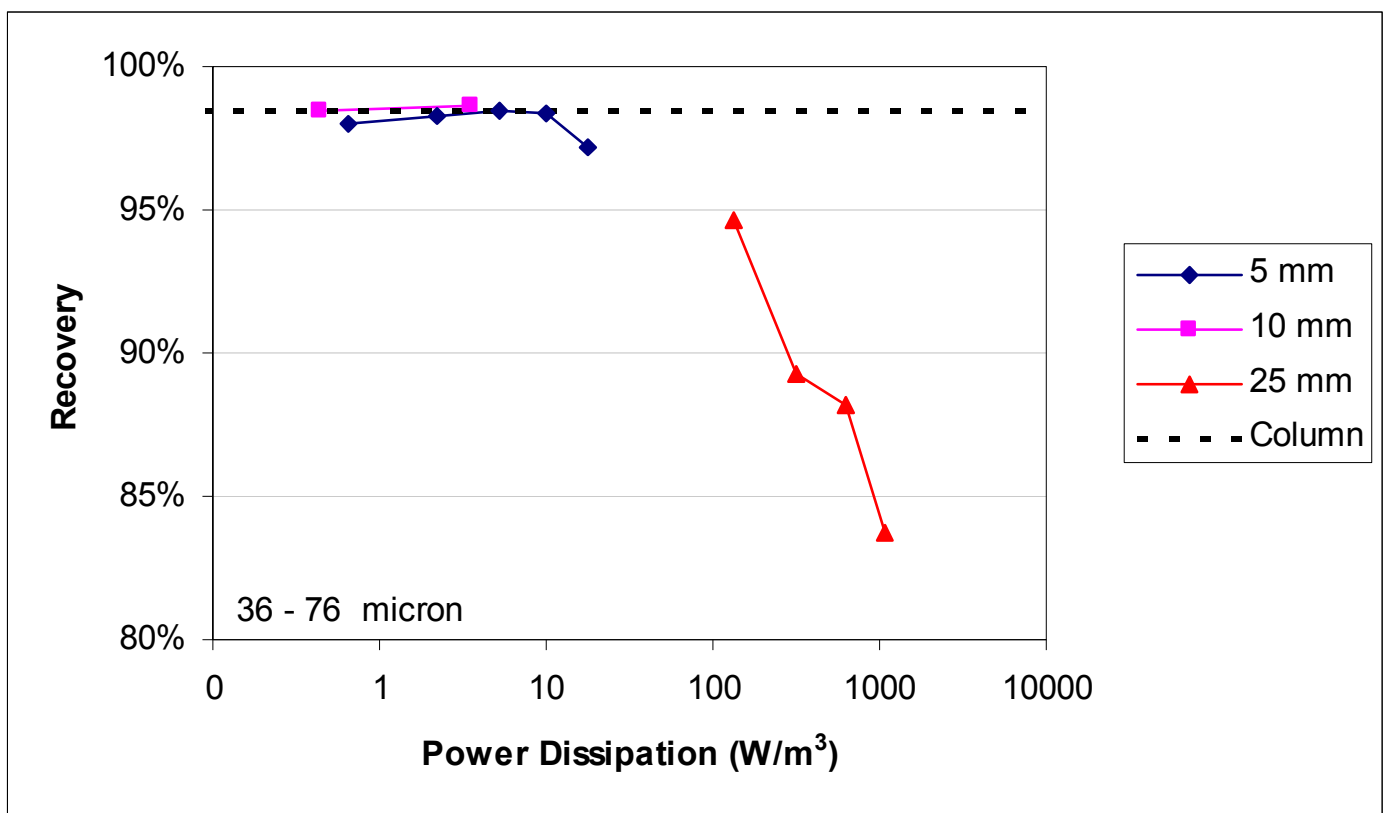

Figure 5.4 - The Effect of Power Dissipation on the Recovery of Weakly Hydrophobic 36 - 76 micron Quartz Particles

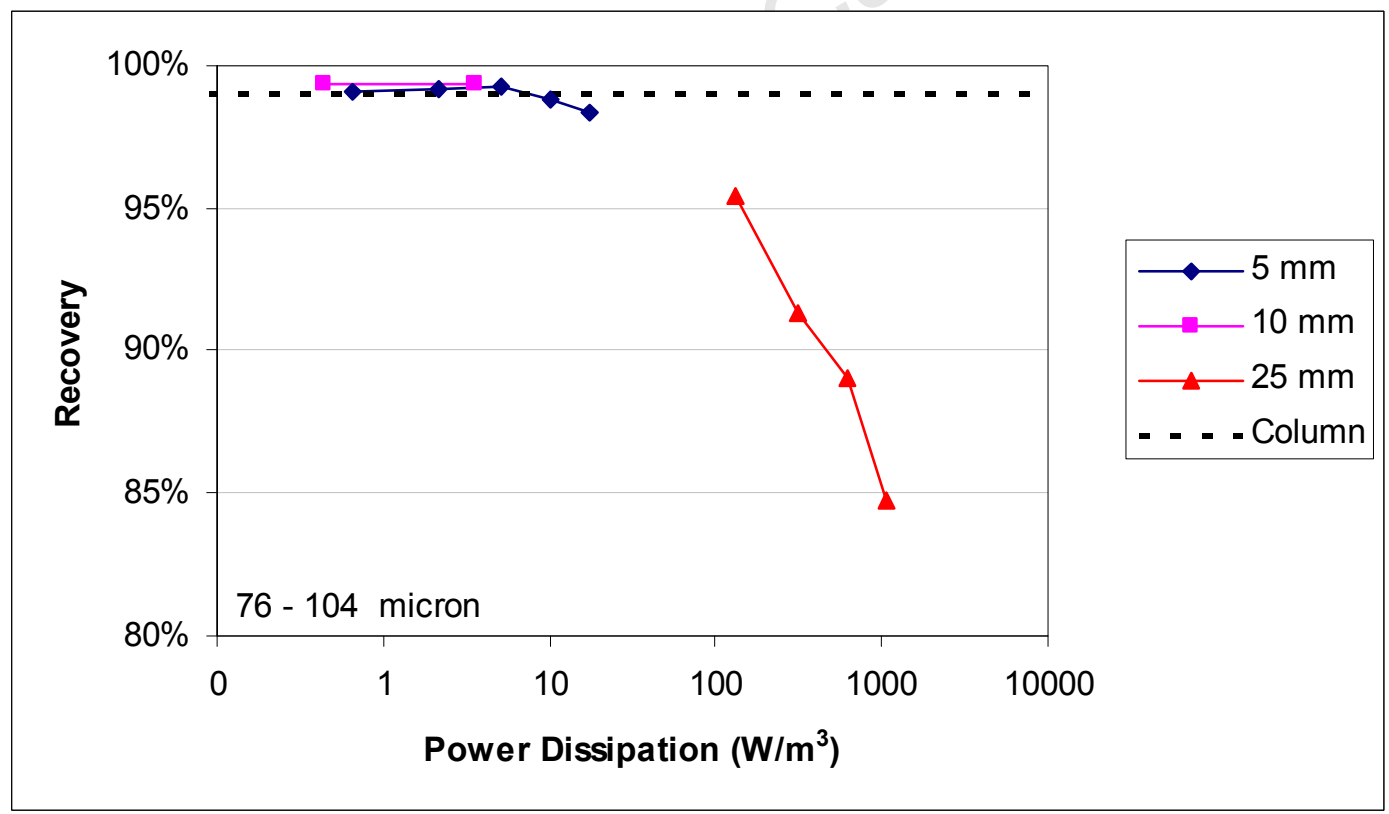

Figure 5.5 - The Effect of Power Dissipation on the Recovery of Weakly Hydrophobic 76 - 104 micron Quartz Particles 
Figures 5.2, 5.3, 5.4 and 5.5 illustrate the effect of power dissipation on the recovery of the various size fractions in the feed, relative to the recovery obtained in a standard column cell. As expected, an increase in power dissipation produces an increase in the recovery of fine particles, with recoveries being improved by up to $8.2 \%$ for the 3 to 17 micron size fraction and up to $5.0 \%$ for the 17-36 micron fraction. This increase is soon followed by a substantial drop in flotation recoveries as power dissipation is further increased due to increased particle-bubble detachment. This effect is even more pronounced for the coarser fractions, where the recoveries remain in the vicinity of the column recoveries for the lower power dissipations and rapidly decrease as the power dissipation is increased. This is in agreement with flotation theory which indicates that coarse particles are more susceptible to detachment effects. This decline in recovery may have been even further exacerbated by the decline in plug flow conditions at higher frequencies and amplitudes, given the high overall recoveries obtained (c.f. Section 4.4).

Surprising to note is the low power dissipation at which particle-bubble detachment starts to dominate. Particle recovery rates appear to be adversely affected by power dissipations as low as $10 \mathrm{~W} / \mathrm{m}^{3}$. This is orders of magnitude less than the power dissipations obtained in most conventional systems which usually range between 700 and $3000 \mathrm{~W} / \mathrm{m}^{3}$. The rapid decline in coarse particle recovery together with the low optimum power dissipation observed, suggests that the improvements in recovery could be substantially increased if the tests were carried out at a higher collector dosage. This would render the particles less susceptible to detachment forces and better able to take advantage of the increased contacting rates which are anticipated at higher power inputs.

The drawback of increasing the collector dosage is that the overall recoveries would be expected to increase despite already being close to $100 \%$ in some size fractions. For this reason, the air rate and cell residence time were also substantially decreased in the second phase of testing (c.f. Table 3.3). 
The reason for the low optimum power input obtained cannot however be solely attributed to particle hydrophobicity. As detailed in Section 3.3, these tests were performed at the same collector surface coverage conditions as previous studies on quartz flotation in a stirred tank (Deglon, 1998). The results of this study indicated an optimal flotation power input in the vicinity of $650 \mathrm{~W} / \mathrm{m}^{3}$ for a Rushton turbine impeller. The reason for this low optimum power input can therefore almost certainly be attributed to differences in the hydrodynamic environment rather than differences in particle hydrophobicity. The possible causes of this difference are further discussed in Section 5.2.2 when examining the moderately hydrophobic flotation data.

\subsubsection{Summary of Findings and Actions Taken}

From the above test work, it was concluded that the OBC operates in a substantially lower energy regime compared with conventional stirred systems. For this reason, the moderately hydrophobic tests were limited to a more focussed power dissipation range of 0 to $250 \mathrm{~W} / \mathrm{m}^{3}$. The flotation results were also found to be highly detachment dominated, indicating that additional collector may be required in order to investigate the relationship between collision rate and power dissipation. This would also bring the study closer within range of other studies conducted at higher particle hydrophobicities (Pyke, 2004, Newell, 2006). Such an increase in hydrophobicity was however likely to further increase the size-by-size recoveries obtained in the cell, making it difficult to extract trends in rate constant. The moderately hydrophobic flotation tests were therefore carried out at a substantially lower air rate and cell residence time in order to decrease the overall recoveries obtained. Lastly, the high recoveries obtained in the weakly hydrophobic tests indicated that mixing effects may have a significant effect on the flotation performance of the OBC. For this reason, the investigation under moderate hydrophobic conditions was coupled with an investigation of the residence time distribution obtained at each flotation setting (c.f. Section 4.4) and the results were combined using a tanks-in-series model to decouple mixing effects from the flotation kinetics. 


\subsection{Flotation Response under Moderately Hydrophobic Conditions}

The following section details the flotation response of quartz as a function of power dissipation under moderately hydrophobic conditions and compares the results with similar studies in stirred tank environments.

\subsubsection{Particle Size vs. Recovery}

Figure 5.6 illustrates the change in flotation response in a standard column as a function of particle size due to the changed experimental conditions between the weakly hydrophobic and the moderately hydrophobic conditions (c.f. Table 3.3). For the moderately hydrophobic conditions, the air flow rate and cell residence time have been substantially reduced.

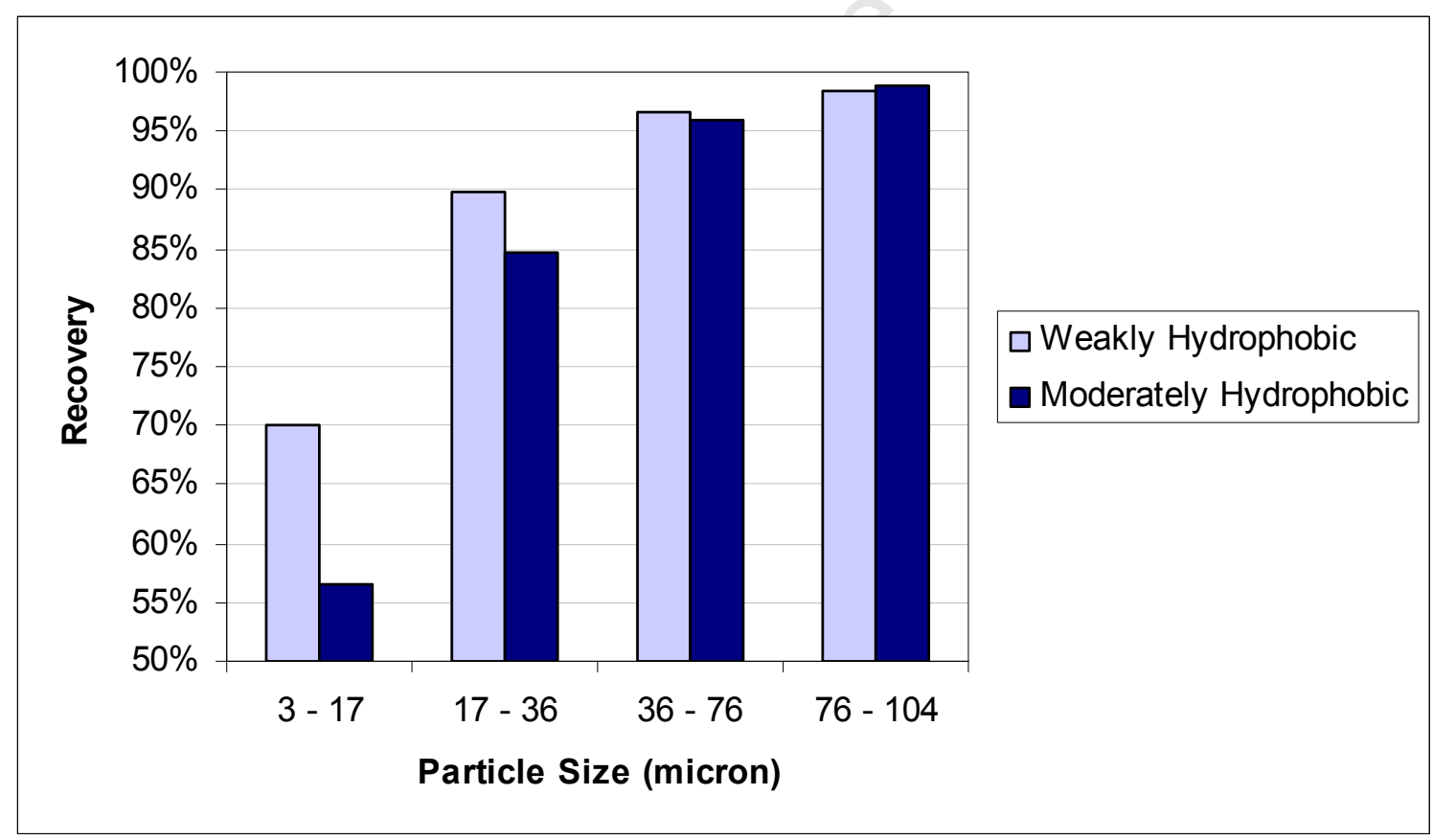

Figure 5.6 - Comparison of Recoveries Obtained in a Standard Column for the Weakly Hydrophobic and Moderately Hydrophobic Conditions with the Cell Residence Time and Air Flow Rate Substantially Reduced (c.f. Table 3.3).

Figure 5.6 shows that the change in flotation conditions was successful in decreasing the size-by-size recoveries obtained, particularly for the finer size fractions. The coarse 
particle recovery however remained in the same vicinity as in the previous test work. Further reduction of the recovery was however not possible since practical limitations prohibited the further reduction of residence time and air flow rate.

Reproducibility tests on the standard column cell did however indicate that good reproducibility of the recovery results was obtained, particularly in the larger size fractions. This improved reproducibility allowed the trends in the coarser size fractions to be distinguished despite the high recoveries. However the error in recovery was found to increase when propagated through to the rate constant, resulting in larger errors. The reproducibility of the tests is shown in Table 5.1:

\section{Table 5.1: Relative Error in Recovery and Rate Constant per Particle Size Class}

\begin{tabular}{|c|c|c|}
\hline Size Fraction & $\begin{array}{c}\text { Relative Std. Deviation in } \\
\text { Recovery }\end{array}$ & $\begin{array}{c}\text { Relative Std. Deviation } \\
\text { in Rate Constant }\end{array}$ \\
\hline \hline $0-3 \mu \mathrm{m}$ & $3.6 \%$ & $8.6 \%$ \\
$3-17 \mu \mathrm{m}$ & $3.0 \%$ & $7.8 \%$ \\
$17-36 \mu \mathrm{m}$ & $1.0 \%$ & $6.6 \%$ \\
$36-76 \mu \mathrm{m}$ & $0.4 \%$ & $6.6 \%$ \\
$76-104 \mu \mathrm{m}$ & $0.2 \%$ & $7.4 \%$ \\
\hline
\end{tabular}

This range of reproducibility was considered acceptable for subsequent test work and the results of flotation tests conducted in the standard flotation column were used as the basis for comparison of results obtained under agitated conditions.

\subsubsection{The Effect of Power Dissipation on the Flotation Recovery}

Figures 5.7, 5.8, 5.9 and 5.10 illustrate the effect of power dissipation on the flotation recovery under moderately hydrophobic conditions. 


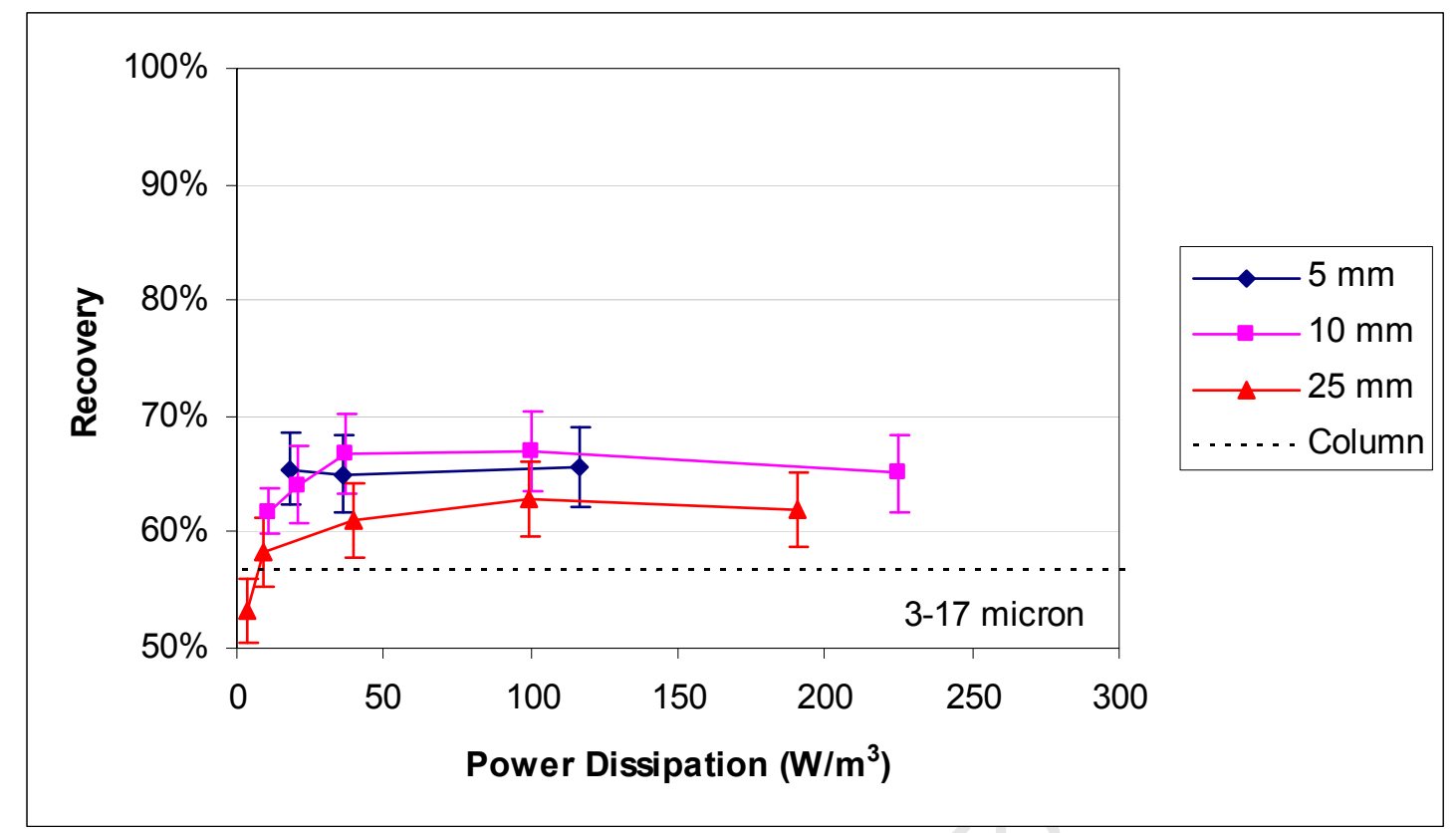

Figure 5.7 - The Effect of Power Dissipation on the Recovery of Moderately Hydrophobic 3 - 17 micron Quartz Particles

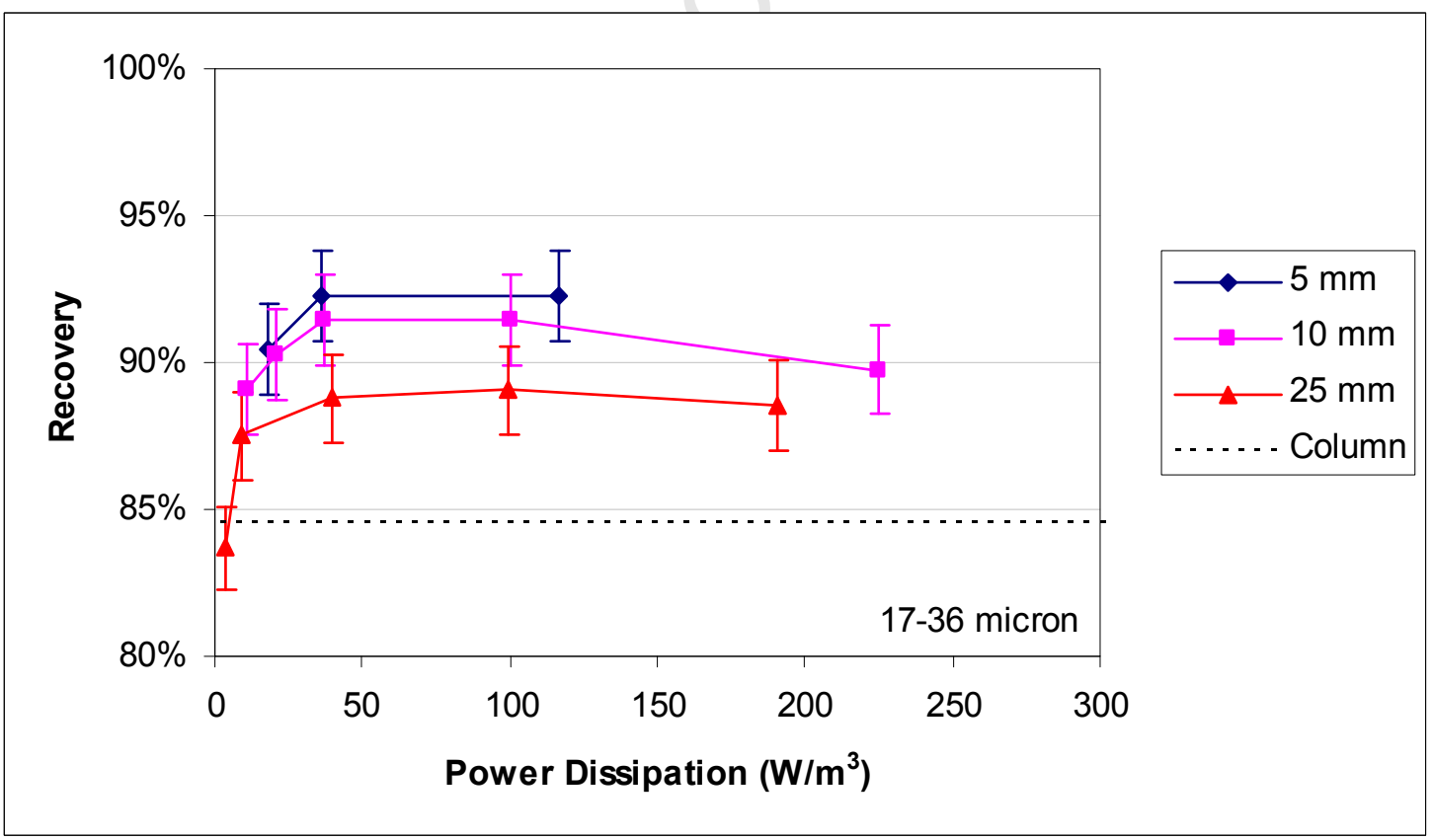

Figure 5.8 - The Effect of Power Dissipation on the Recovery of Moderately Hydrophobic 17 - 36 micron Quartz Particles 


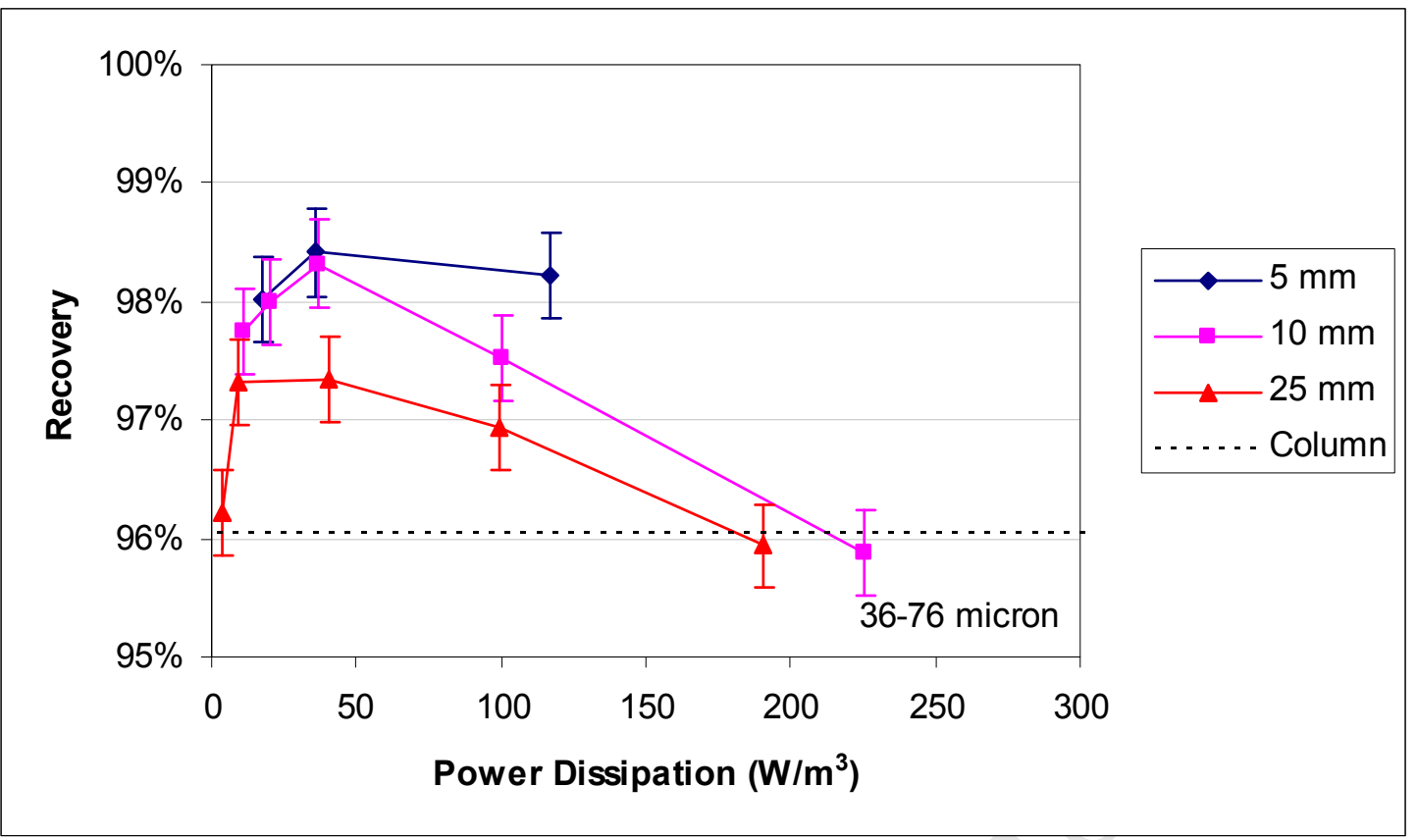

Figure 5.9 - The Effect of Power Dissipation on the Recovery of Moderately Hydrophobic 36 - 76 micron Quartz Particles

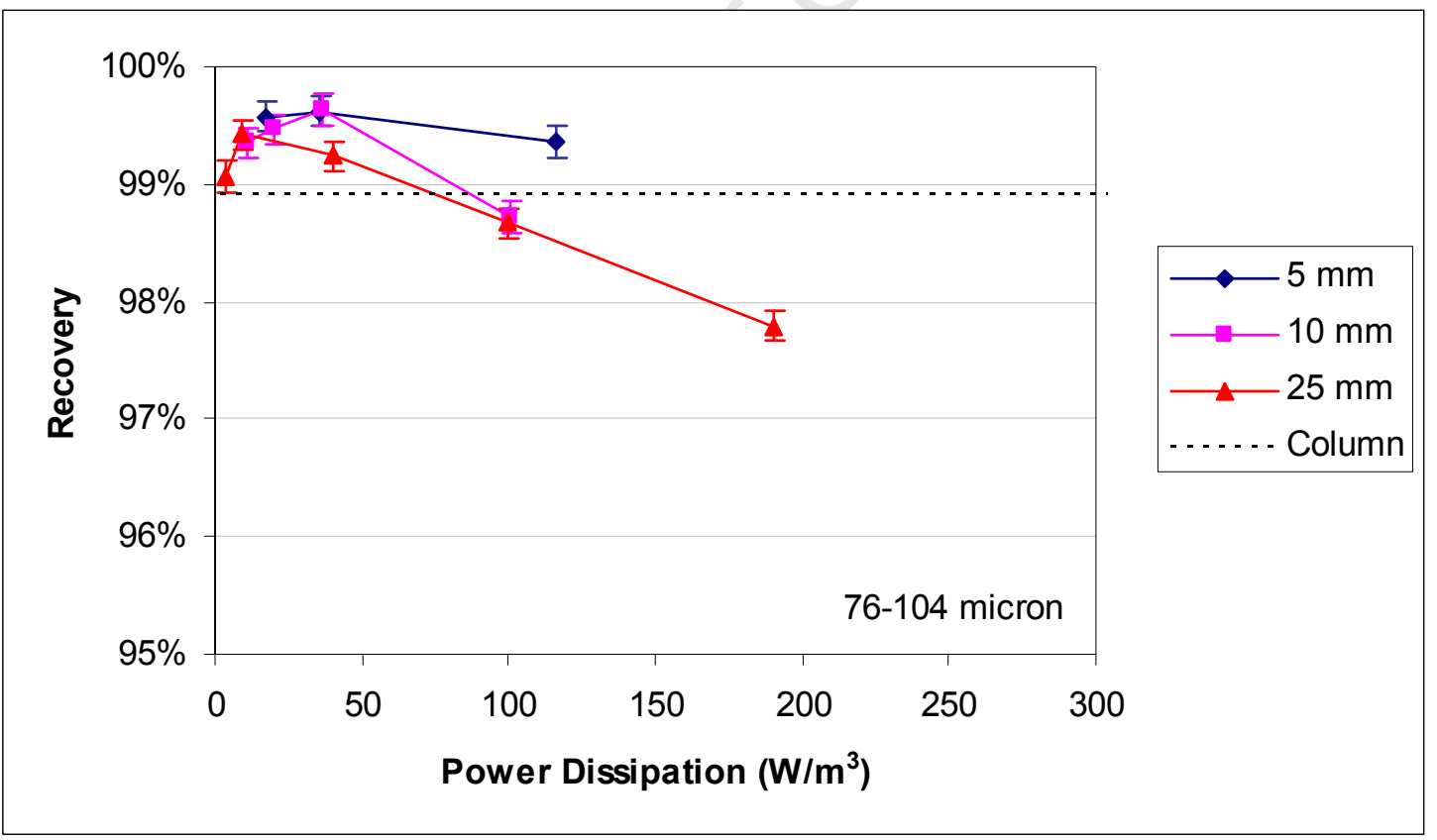

Figure 5.10 - The Effect of Power Dissipation on the Recovery of Moderately Hydrophobic 76 - 104 micron Quartz Particles 
Figure 5.7, 5.8, 5.9 and 5.10 illustrate that the $\mathrm{OBC}$ was able to improve the flotation recovery relative to a standard flotation column for all particle size fractions. Recoveries increased with increasing power dissipation, particularly in the finer particle fractions where recoveries were improved by up to $10.4 \%$ in the $3-17$ micron size fraction and up to $7.7 \%$ in the 17-36 micron size fraction. This increase in flotation recovery occurred at a decreasing rate and levelled off between 40 and $100 \mathrm{~W} / \mathrm{m}^{3}$ in the fine particle size fractions due to increased detachment forces. For particles greater than 36 microns, these detachment forces were significant and resulted in large decreases in recovery. This optimum flotation power dissipation for moderately hydrophobic conditions was considerably larger than the optimum of $10 \mathrm{~W} / \mathrm{m}^{3}$ observed under weakly hydrophobic conditions and illustrates the substantial effect of particle hydrophobicity on the particle-bubble detachment rate.

The flotation response appears to be similar for the three amplitudes of oscillation tested, although slightly higher recoveries were obtained at low amplitude. This effect is believed to arise from a mixing effect, since enhanced plug flow conditions were obtained at lower amplitudes as shown in Figure 4.13 in Section 4.4.

Therefore, to further interrogate the data, the results of the moderately hydrophobic flotation tests were combined with the mixing data from Section 4.4 using the tanks-inseries model to account for any mixing effects at high recovery. The results of this analysis are detailed in Section 5.3.

\subsection{Rate Constants Obtained Using the Tanks-in-Series Model}

Figure 5.11 shows the effect of power dissipation on the flotation rate of moderately hydrophobic quartz particles at an oscillatory amplitude of $10 \mathrm{~mm}$. 


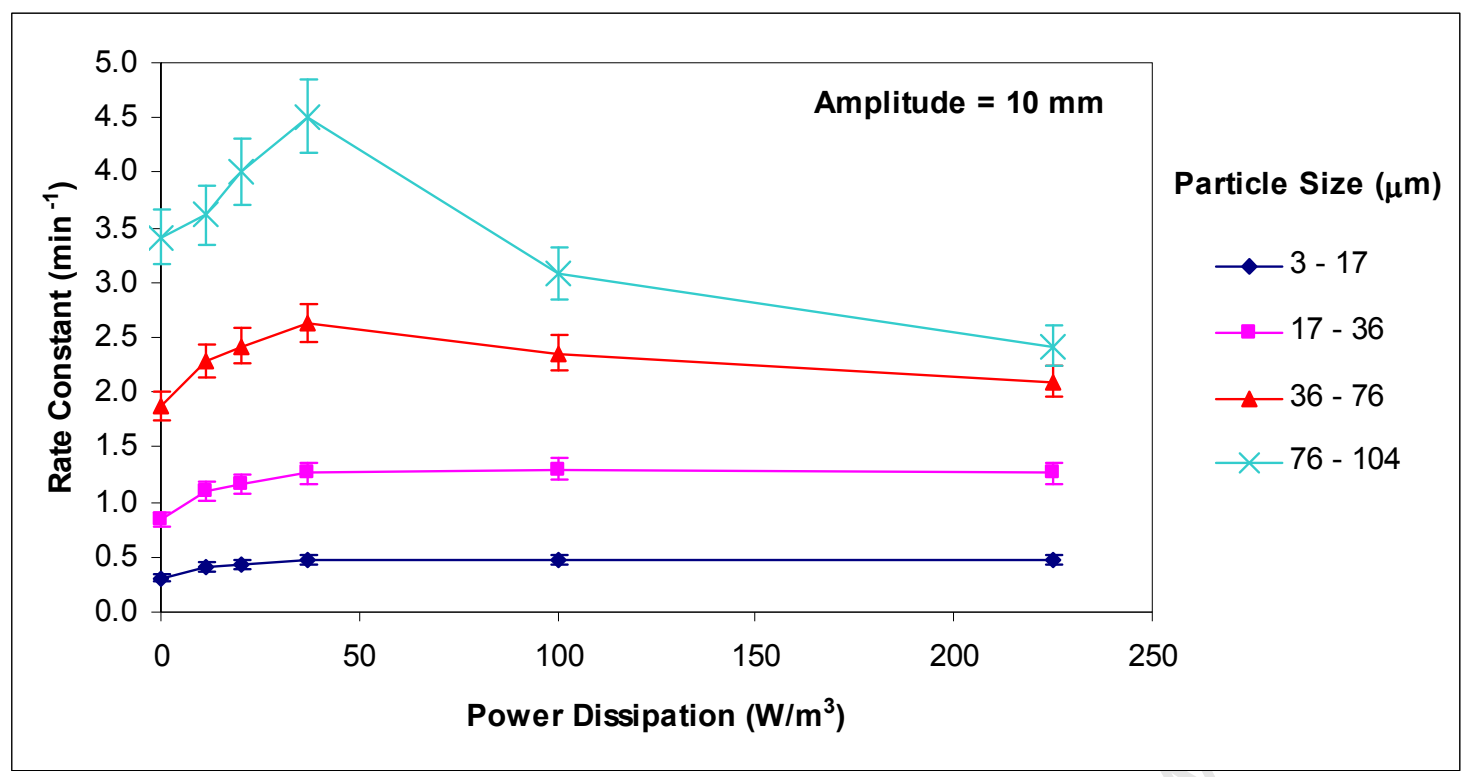

Figure 5.11 - The Effect of Power Dissipation on the Flotation Rate Constant for Moderately Hydrophobic Quartz Particles at an Oscillation Amplitude of $10 \mathrm{~mm}$

Figure 5.11 illustrates that the addition of power dissipation to the system was able to produce significant increases in the flotation rate constant for all the particle size fractions in the feed. As expected, the flotation rate of the larger size fractions was greater than that obtained in the finer size fractions since coarser particles have a greater probability of interception with flotation bubbles due to their increased size and inertia. This is consistent with the findings of numerous other studies which have shown an increase in the rate constant with increasing particle size, for this particle size range (Ahmed \& Jameson, 1985, Deglon, 1998, Pyke, 2004, Newell, 2006).

Although the coarser size fractions exhibited a greater overall increase in the rate constant, the relative increase in rate constant with increasing power dissipation appears to be greater for the finer particles size fractions. To further discern this point, the flotation rate of each particle size class is plotted relative to the rate constant obtained in a standard flotation column, so as to provide a basis for comparison between size fractions. Thus the trends in Figures 5.12, 5.13 and 5.14 illustrate the relative change in the rate constant from a quiescent system to an agitated system. 


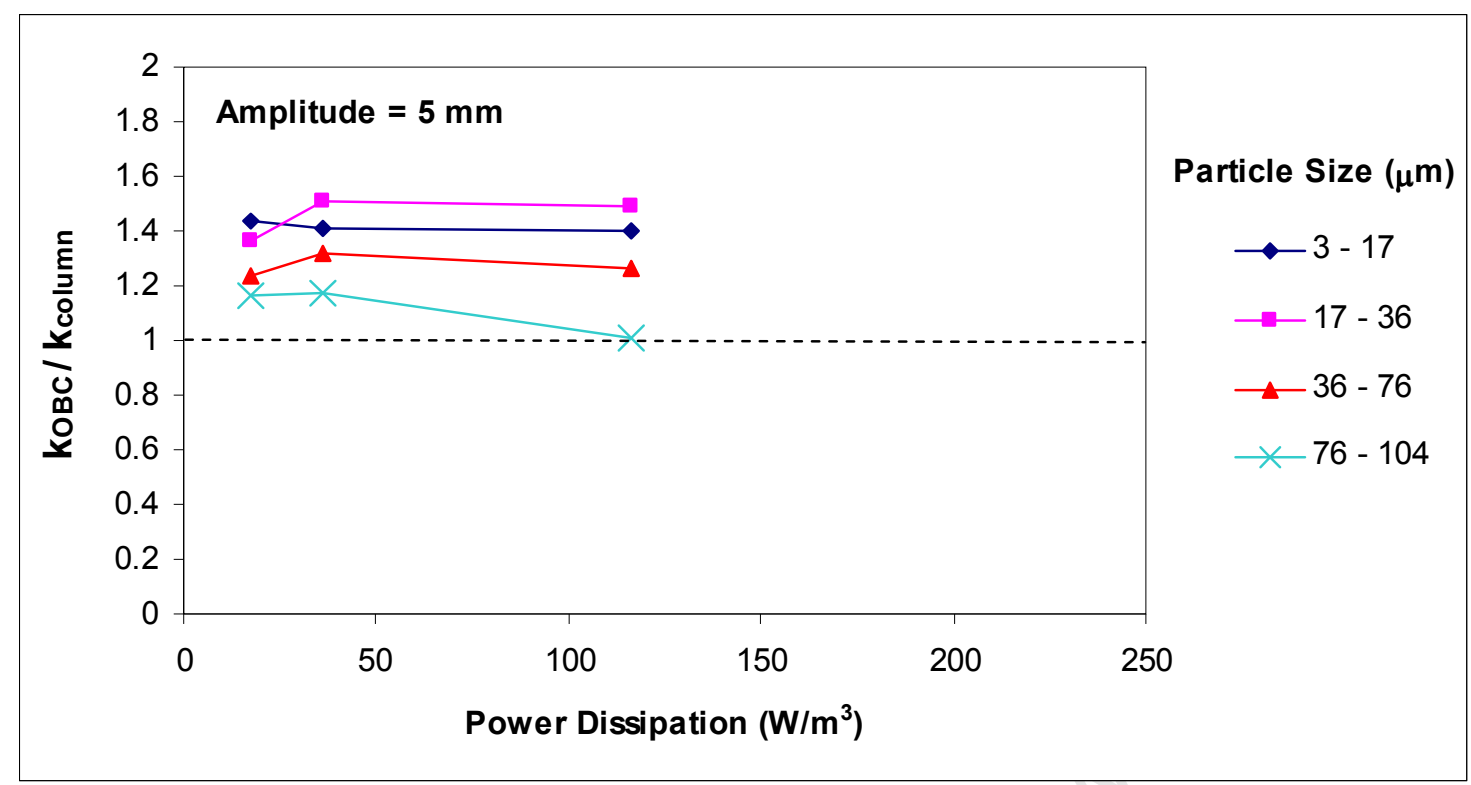

Figure 5.12 - Flotation Response Moderately Hydrophobic Quartz at an Amplitude of $5 \mathrm{~mm}$ using the Tanks-in-Series Model

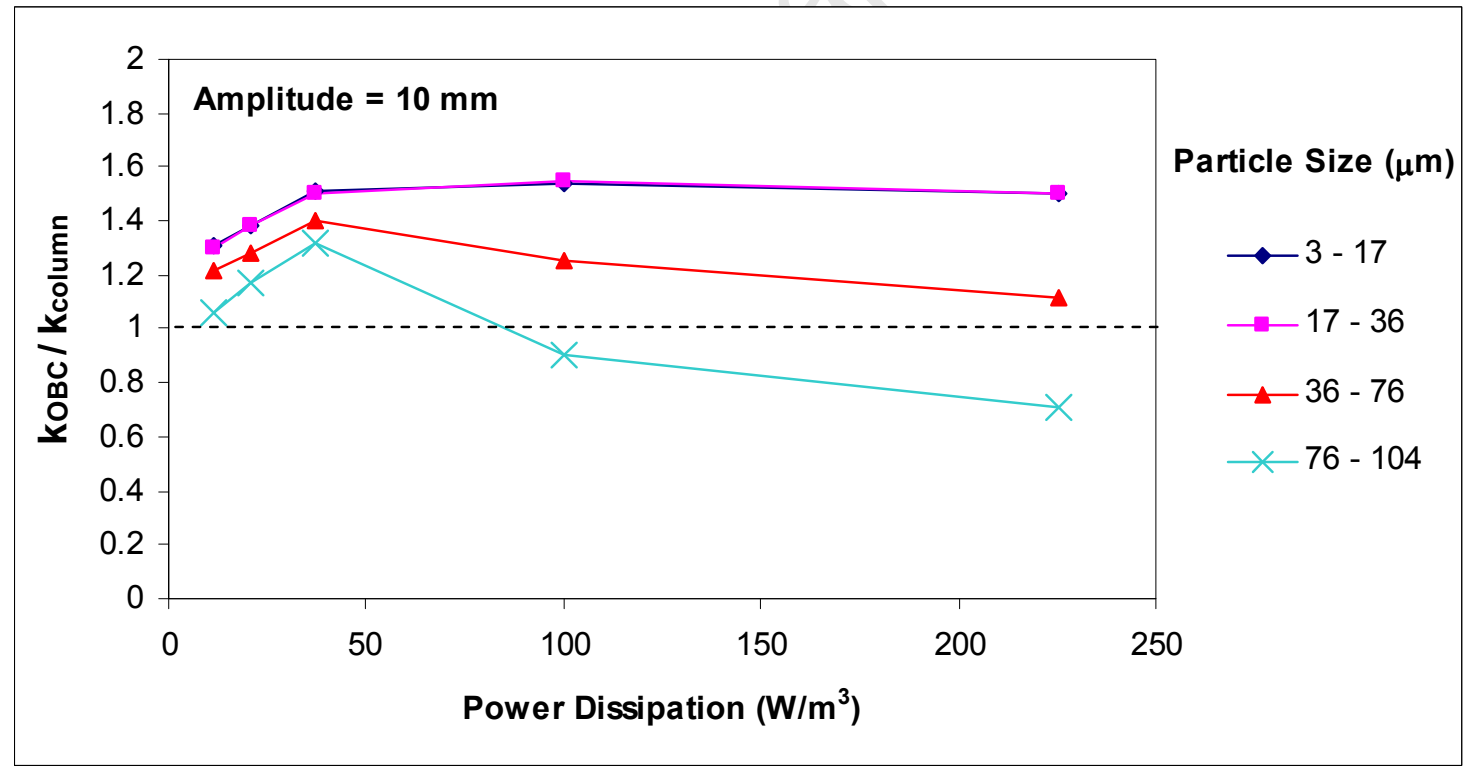

Figure 5.13 - Flotation Response Moderately Hydrophobic Quartz at an Amplitude of $10 \mathrm{~mm}$ using the Tanks-in-Series Model 


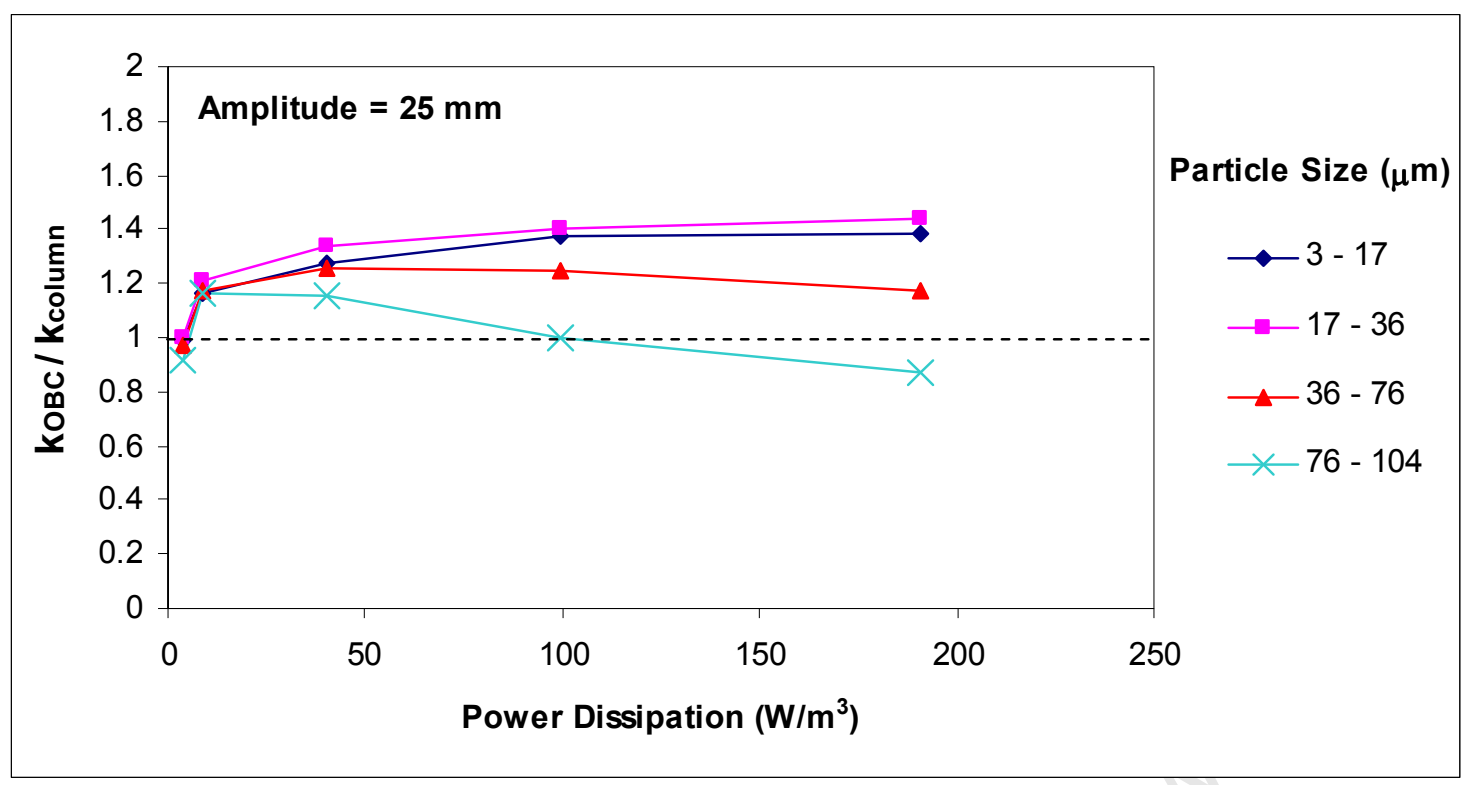

Figure 5.14 - Flotation Response Moderately Hydrophobic Quartz at an Amplitude of $25 \mathrm{~mm}$ using the Tanks-in-Series Model

The trends in Figures 5.12, 5.13 and 5.14 show a similar initial increase in the flotation rate with increasing power input relative to a standard flotation column for all particle size classes. The flotation rate increased at a decreasing rate and levelled off for the finer size fractions. For the larger $36-76$ micron and 76 - 104 micron size classes, the rate started to decrease at approximately $50 \mathrm{~W} / \mathrm{m}^{3}$, due to increased particle-bubble detachment. This was as expected since large particles are more susceptible to detachment forces (Schulze, 1993).

The relative improvement in the flotation rate with increasing power dissipation was found to be greatest for the finer size fractions which were improved by approximately $60 \%$ relative to a standard flotation column. More conservative improvements in the vicinity of $30 \%$ to $40 \%$ were observed for the $36-76$ and $76-104$ micron size fractions. This is due to the increased probability of detachment of these particles, which is believed to have hindered any further improvements in flotation rate with increased power dissipation. 
It should be noted however, that even the flotation rate of the finest size fraction was found to level off after $50 \mathrm{~W} / \mathrm{m}^{3}$, most likely due to increased detachment of these fine particles from flotation bubbles. This finding is contrary to the widespread belief that fine particles are insusceptible to detachment forces due to their small size and inertia.

With the mixing effects removed, the trends obtained in terms of flotation rate and power input are largely consistent between different amplitudes of oscillation, with no statistically significant differences between them. This shows that the primary benefit of varying the amplitude of oscillation lies in the mixing characteristics obtained rather than the particle-bubble contacting rate. A change in amplitude was also linked to a change in the ratio of inertial to frictional forces in the fluid (c.f. Section 4.1.1.1). However, Figures 5.12, 5.13 and 5.14 show that the inertial forces under oscillatory flow in fact have little effect on the flotation kinetics in the system. This suggests that the shear rate in the cell is similarly distributed through the cell for this range of amplitudes. The kinetics of the system are therefore considered to be dominated by the average power input to the cell.

The results of this study are also in reasonable agreement with previous studies on hydrodynamics and particle-bubble contacting in Rushton turbine impeller systems, although the point at which detachment starts to dominate the kinetics is considerably lower. For example, Deglon (1998) investigated the flotation response of quartz as a function of hydrodynamic factors such as power input and bubble size for a 2.25 litre batch flotation cell. For the same collector conditions as the low hydrophobicity setting in this study (c.f. Section 5.1), an increase in kinetics was obtained when increasing power input from $100 \mathrm{~W} / \mathrm{m}^{3}$ to $650 \mathrm{~W} / \mathrm{m}^{3}$, for both sub 32 micron and sub 100 micron quartz particles. As power input was further increased, the rate constant decreased sharply in both size fractions resulting in an optimum flotation power input. This optimum power input was noted to be lower than that required for effective bubble break-up and it was therefore suggested that the energy conditions required for optimal flotation performance were significantly lower than that required to produce small bubbles. These findings are in agreement with the findings of the current study although 
the optimal power conditions identified are approximately one order of magnitude higher in the Rushton turbine cell relative to the $\mathrm{OBC}$.

Similarly Pyke (2004) investigated the effect of agitation and hydrophobicity on the flotation of quartz, chalcopyrite and galena particles in a Rushton turbine cell. Results indicated that when the contact angle of quartz was increased from $73^{\circ}$ to $80^{\circ}$ (advancing contact angle) at a constant power input of $450 \mathrm{~W} / \mathrm{m}^{3}$, the overall rate of flotation increased dramatically for all particle sizes. This effect was most apparent in the coarser particle flotation rate, since the increase in hydrophobicity rendered them less susceptible to detachment. When increasing the power input from $450 \mathrm{~W} / \mathrm{m}^{3}$ to $1450 \mathrm{~W} / \mathrm{m}^{3}$, the flotation rate of quartz particles with an advancing contact angle of the $73^{\circ}$ decreased due to increased detachment for all particle size fractions. This indicates that for particles of this hydrophobicity, the system was largely detachment dominated. When the hydrophobicity was increased to $80^{\circ}$, the flotation rates of the sub 30 micron particles remained constant and only the rate of the coarser particles decreased as power input was increased from 450 to $1800 \mathrm{~W} / \mathrm{m}^{3}$, due to detachment. This shows that hydrophobicity has a large effect on the detachment rate of particles, particularly as particle size increases. These findings are in agreement with the findings of the current study which found a definite shift in optimum flotation conditions with increased particle hydrophobicity, even though the hydrophobicities tested in the work of Pyke (2004) were significantly higher. Once again optimum flotation conditions in the Rushton turbine cell appear to be an order of magnitude higher than those obtained in the $\mathrm{OBC}$.

One study which shows an even greater discrepancy in optimum power input is the work of Newell (2006), which investigated the effect of energy on quartz flotation in 3 different size flotation cells driven by Rushton turbines. The investigation centred on power inputs between 1.25 and $12.6 \mathrm{~kW} / \mathrm{m}^{3}$. He found that for quartz with a contact angle of $80^{\circ}$, the rate constant increased steadily with energy up to $4.1 \mathrm{~kW} / \mathrm{m}^{3}$ before remaining constant until $12.6 \mathrm{~kW} / \mathrm{m}^{3}$. These trends obtained with power input are remarkably similar to those reported in Figures 5.12, 5.13 and 5.14 where the flotation rate of the finer size fractions levelled off with added power input. The findings of 
Newell (2006) however indicate that optimal flotation performance is obtained somewhere between 4.1 and $12.6 \mathrm{~kW} / \mathrm{m}^{3}$. This value would be an order of magnitude higher than the values obtained by Deglon (1998) and Pyke (2004) and two orders of magnitude higher than the current study. The reason for this discrepancy is largely unclear, however calculation shows that there is a large discrepancy between the power numbers obtained by Deglon (1998) and Newell (2006), which may account for the significant difference in the specific power inputs calculated.

Thus the trends of this study are largely in agreement with previous studies on energy and quartz flotation, however the point at which detachment starts to dominate the kinetics is substantially lower. This point is also orders of magnitude lower than the power input to most industrial flotation cells, which generally range between 700 and $3000 \mathrm{~W} / \mathrm{m}^{3}$. There are many factors which can be attributed to this large difference and each will be discussed in turn.

\subsubsection{Hydrophobicity of the Material}

Although the range of collector dosages tested represents a broad range of collector dosages for the quartz-amine system (Smith \& Scott, 1990), it is believed that the hydrophobicity of this material was fairly low compared with other studies which have used various methylation techniques in order to render the quartz particles hydrophobic (Pyke, 2004, Newell, 2006). Using these methylation techniques, particle contact angles of up to $80^{\circ}$ can be obtained, which are believed be more representative of the fast floating material in mineral slurries. For this study, a lower range of hydrophobicities was considered more appropriate in order to simulate the response of slow floating particles in mineral slurries, which are difficult to recover using conventional flotation technologies.

As can be seen when comparing the low and moderate hydrophobicity flotation recoveries, a large change in the optimum power dissipation was observed with increasing hydrophobicity (c.f. Figure $5.2 \&$ 5.7). Thus, this difference in the hydrophobicity could have accounted for the lower optimum power dissipation that was 
observed. However, the work of Deglon (1998) was performed under the same collector surface coverage conditions as the weakly hydrophobic conditions tested in this study. The results still showed the optimum power input in a Rushton turbine system to be an order of magnitude higher than that found in the OBC. Thus the discrepancy in the optimum power input can almost certainly be attributed to the innate differences in the hydrodynamics of the OBC compared with those of a Rushton turbine cell, rather than differences in particle hydrophobicity.

\subsubsection{Non-Homogeneous Distribution of Shear Rate in Conventional Cells}

It is well known that while conventional impeller-driven cells do produce relatively large average power inputs to the cell, this energy is largely concentrated in a small region around the impeller. This leads to exceptionally high shear rates near the impeller which drop off sharply as one moves away from the impeller. Studies on the energy dissipation rate in Rushton turbine flotation cells have shown that the energy dissipation rate close to the impeller can be 30 to 40 times greater than that in the bulk of the cell (Deglon, 1998, Koh \& Schwarz, 2003). In contrast, the OBC has been found to produce a reasonably even distribution of shear rates in the fluid (c.f. Section 2.2.4). Furthermore, CFD simulations by Chew et al. (2004) indicate that particles in an OBC spend the majority of their residence time in the regions of relatively high shear in the fluid, possibly due to vortex trapping, while in a stirred tank, particles reside mainly in regions of considerably lower shear rates. This means that although the average power input to a conventional cell is high, particles spend the majority of their residence time in lower shear regions of the cell, where energy dissipation rates may be of the same order as those found in the OBC. In contrast, particles in an $\mathrm{OBC}$ spend most of their time in higher shear regions where they are able to take advantage of higher contacting rates at lower power inputs. This, together with the even distribution of shear, would suggest that the $\mathrm{OBC}$ is in fact an inherently more energy efficient contacting device which is better able to optimise the range of shear rates to which particles are exposed. This allows the $\mathrm{OBC}$ to achieve improvements in the contacting rate at lower overall power inputs than in Rushton turbine systems. 
Also important to note is that for most studies on energy in Rushton turbine impeller systems, the optimum power input was still found to lie well below the range of industrial flotation cells which does suggest that the energy conditions required for optimal particle-bubble contacting are significantly lower than those required to produce small bubbles.

\subsubsection{Oscillatory Nature of Flow in an OBC}

In contrast to a conventional cell, agitation in an OBC is achieved by oscillating the fluid through a baffled tube. This means that the fluid is in a constant state of acceleration and deceleration, resulting in an additional component of fluid velocity which oscillates approximately sinusoidally with time. This additional component of fluid flow is not present in a conventional cell where the fluid flow is largely at steady state and the only deviation about the mean flow velocity is a turbulent fluctuating velocity, developed as a result of turbulent kinetic energy in the system. This concept is illustrated in the following figure:

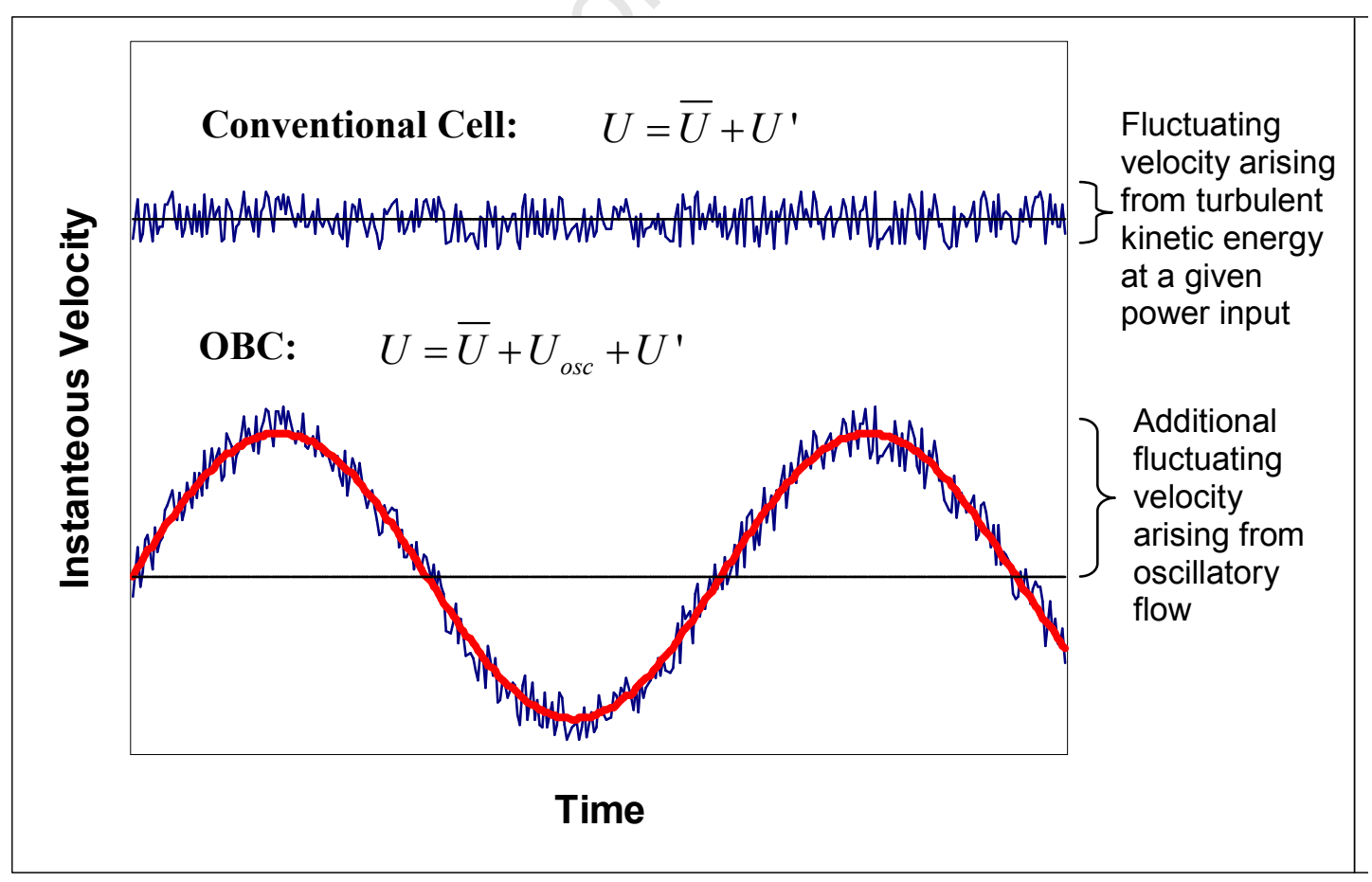

Figure 5.15 - Schematic of Fluctuating Velocity in an OBC and in a Conventional Cell 
Figure 5.15 shows a schematic of the instantaneous fluid velocity that would be measured in both a conventional cell and an OBC. In a conventional cell, the velocity is made up by a mean and a fluctuating component. In the $\mathrm{OBC}$, the same two components are present, along with a third component due to oscillatory flow.

Up to this point, the analysis of the flotation data has been conducted on the basis of the average energy dissipation rate in the system, which gives an indication of the intensity of turbulence in the cell. However, as was shown in section 2.3, the effect of hydrodynamics on flotation kinetics is usually characterised by both the energy dissipation rate in the system and the mean squared fluctuating velocity of the fluid. Thus, although the mean power dissipation in the OBC may be very low at the flotation conditions tested, the oscillatory component of the flow under these conditions gives rise to a substantial increase in the root mean squared fluctuating velocity of the fluid. This is illustrated in Figure 5.16 where values of root mean squared velocity are calculated as a function of power input. Calculations are made only on the oscillatory component of flow and are not based on estimates of the fluctuating velocity due to turbulence (U'). Thus these root mean squared velocities occur in addition to any fluctuating velocities arising from conventional turbulence in the fluid and represent an 'apparent' root mean squared velocity to which particles are exposed. 


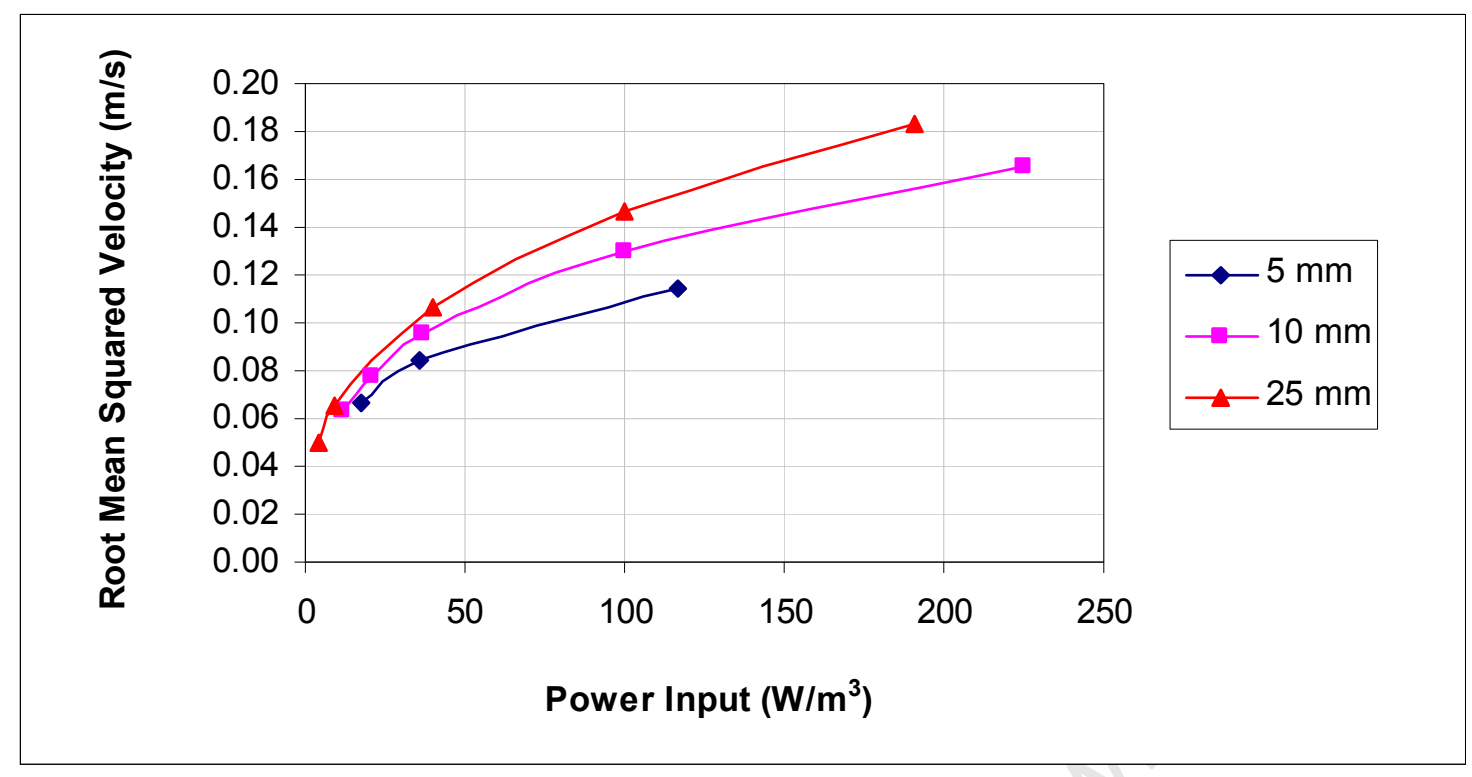

Figure 5.16 - Root Mean Squared Fluid Velocities Arising from Oscillatory Flow in the $O B C$ as a Function of the Mean Power Input

Figure 5.16 illustrates the significant increases in the root mean squared fluctuating velocity which occur as a result of oscillatory flow. As a point of comparison, Deglon (1998) found that the r.m.s. fluctuating velocity in a Rushton turbine cell operating at a specific power input of $2100 \mathrm{~W} / \mathrm{m}^{3}$ was approximately $0.20 \mathrm{~m} / \mathrm{s}$ in the bulk of the cell and up to $0.53 \mathrm{~m} / \mathrm{s}$ around the impeller. Figure 5.16 shows that the fluctuating velocity achieved by oscillatory flow can reach up to $0.18 \mathrm{~m} / \mathrm{s}$ at a power input of just 190 $\mathrm{W} / \mathrm{m}^{3}$. This additional 'apparent' fluctuating velocity under oscillatory flow is speculated to have a significant effect on the particle-bubble contacting rate since it contributes to a higher relative velocity between particles and bubbles in the system.

Figure 5.17 shows the relationship between the mean squared fluctuating velocity due to oscillatory flow and the flotation rate constant of the 17 - 36 micron size fraction for a variety of amplitudes of oscillation. 


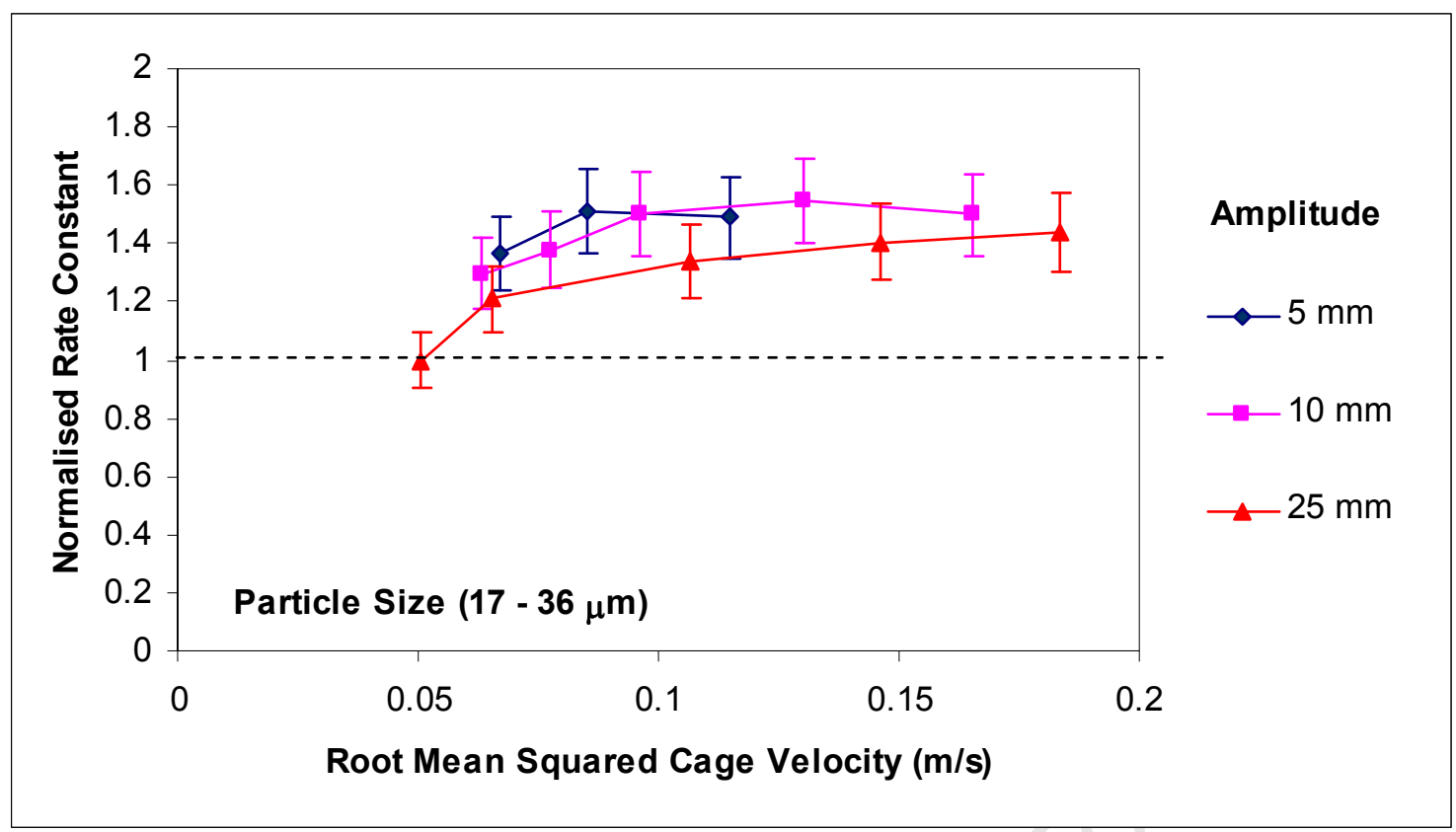

Figure 5.17 - Normalised Rate Constant of the 17-36 micron Quartz Particles as a Function of the Root Mean Squared Baffle Cage Velocity

Figure 5.17 shows that the relationship between root mean squared oscillatory velocity and the flotation rate constant is very similar to trends obtained in terms of mean power input, and increases at a decreasing rate. However, unlike the mean energy dissipation rate, the range of r.m.s. velocities obtained is within range of those found within a the Rushton turbine systems, despite the significantly lower power dissipation in the cell. It is this increased r.m.s. fluctuating velocity under oscillatory flow which is believed to result in the high collision and detachment rates observed at power dissipations an order of magnitude lower than those found in a conventional cell.

As shown in Section 2.3, the model of Abrahamson (1975) predicts an approximately linear relationship between the rate constant and the r.m.s. fluctuating velocity, depending on the model used to describe the particle and bubble fluctuating velocity (c.f. Equation 2.42). This linear increase is not apparent in the flotation rate data unless all the curvature in the trends is attributed to detachment. It is believed to be unlikely that the Abrahamson model would describe the flotation behaviour in this system in terms of fluctuating velocity since it is derived for the inertial sub-range of turbulence 
which would not form at these low power dissipations. Furthermore, the Abrahamson model assumes a Gaussian distribution of fluctuating velocities which is unlikely to be valid in the current system where the fluctuating velocity distribution is dominated by a single frequency. It should also be noted that the oscillatory component of fluctuating velocity occurs as a result of the bulk fluid flow characteristics of the cell rather than the micro-turbulent environment. The effect of bulk fluid hydrodynamics on the flotation rate has yet to be considered in the derivation of the various fundamental flotation models.

One way to test whether the flotation kinetics are indeed controlled by micro-turbulence (i.e. the energy dissipation rate and the fluctuating velocity due to turbulence), or by the bulk fluid characteristics (i.e. the large scale oscillatory fluctuating velocity), would be to assess the change in flotation kinetics as a function of viscosity. As detailed in section 2.3, flotation theory suggests that the viscosity plays an important role in determining the effect of energy on particle-bubble contacting. The ratio of energy dissipation rate to viscosity determines both the scale of turbulence in the fluid, the particle Stokes number, and ultimately the particle-bubble collision rate through the various fundamental models (Saffman \& Turner, 1956, Abrahamson, 1975, Liepe \& Mockel, 1976). Thus if the micro-turbulent environment is indeed controlling the flotation kinetics, as predicted by flotation theory, a change in viscosity would produce a pronounced change in kinetics. In contrast, if the large scale fluctuating velocity due to oscillatory flow is controlling kinetics, a change in viscosity would have little effect, since studies have shown that the flow patterns in the $\mathrm{OBC}$ are largely insusceptible to changes in viscosity for $\mathrm{Re}_{\mathrm{o}}>1000$ (Fitch et. al., 2005). This possibility will be further assessed when analysing the results of flotation tests performed on highly viscous nickel ore slimes in Chapter 6.

\subsection{The Effect of Bubble Size \& Gas Hold-up on Flotation Kinetics}

In Chapter 4, the effect of oscillation on bubble size and gas hold-up was evaluated at a number of power dissipation conditions. Results indicated that the bubble size was largely constant for all power dissipations apart from a small increase in the measured 
bubble size at low power dissipation due to coalescence of larger bubbles behind the oscillating baffles. This increase was speculated to have an insignificant effect on flotation kinetics since the measured bubble size was the result of several stages of coalescence at each baffle and was therefore not considered to be an accurate representation of the mean bubble size in the agitated zone. It was noted however, that if the bubble size had increased, a decrease in kinetics would have be observed at low power input relative to a standard column. Figures 5.12 and 5.13 appear to show little evidence of such a decrease although Figure 5.14 does show a marginal decrease in the normalised rate constant at a power dissipation of $4.0 \mathrm{~W} / \mathrm{m}^{3}$. This slight decrease may have been due to a coalescence effect but overall the results seem to indicate that bubble size had little effect on the flotation kinetics over the range of power dissipations tested. The assumption of a constant bubble size as a function of power dissipation is therefore considered to be valid.

In terms of gas hold-up, the OBC produces a large increase in gas hold-up as the power dissipation is increased, as was shown in Figure 4.13. According to the models detailed in Section 3.2, the flotation rate constant is directly proportional to the number of bubbles per unit volume in the system and therefore directly proportional to the gas hold-up in the system. Thus the 3.7 fold increase in gas hold-up from $1.2 \%$ for a standard column to $4.4 \%$ at a power input of $225 \mathrm{~W} / \mathrm{m}^{3}$, should have produced a 3.7 fold increase in the number of bubbles per unit volume and therefore a 3.7 fold increase in the rate constant. Examination of Figure 5.12, 5.13 and 5.14 clearly show that this predicted increase is well above the observed increase of 1.5 fold, indicating that gas hold-up is unlikely to have affected the kinetics as postulated in equation 2.32.

Indeed, to the author's knowledge, the linear relationship between rate constant and the number of bubbles per unit volume which is postulated by the fundamental flotation models (c.f. Equation 2.32) has yet to be confirmed experimentally. In fact, the results of Deglon (1998) showed a sharp decrease in the rate constant despite substantial increases in the gas hold-up. Rather, the most commonly quoted relationship between the flotation rate constant and gas phase characteristics is the linear relationship between bubble surface area flux and flotation rate (Gorain et. al., 1997). This 
relationship has since been widely verified in several flotation applications (Franzidis \& Manlapig, 1999, Deglon, 2002, Harris et. al., 2002). In the case of the OBC, the bubble surface area flux in the system was held constant during flotation and therefore the effect of the gas phase on the flotation kinetics is assumed to be constant. This is supported by the flotation data in Figures 5.12, 5.13 and 5.14 which indicates no obvious dependency on gas hold-up. The flotation kinetics in the $\mathrm{OBC}$ are therefore concluded to be independent of the number of bubbles per unit volume in the cell.

This finding is however in disagreement with the behaviour predicted by the various fundamental flotation models. The reason for this discrepancy is thought to be that the fundamental models are derived based on a unit volume of homogeneous isotropic turbulence in which bubbles and particles have no nett velocity. However, the reality in most flotation systems is that the particles, and particularly the bubbles, are continually rising through the unit volume in a state of flux. This means that the number of collisions per unit volume may be more controlled by the rate at which bubbles flux through the unit volume than by the shear number density of bubbles within the system. Up until this point, this effect has not been considered in the development of turbulent fundamental flotation models. This defines a task for future research.

\subsection{Summary of Findings and Implications}

The flotation response of quartz has been studied in the $\mathrm{OBC}$ as a function of the overall power dissipation in the system and the amplitude of oscillation. Results were found to be in good agreement with the results of previous studies with respect to power input. Optimal flotation conditions observed in the OBC were however found to be an order of magnitude lower than those observed in previous studies on Rushton turbine cells. The reason for this discrepancy can almost certainly be attributed to innate differences in hydrodynamics between the $\mathrm{OBC}$ and conventional stirred systems rather than differences in particle hydrophobicity.

Based on the findings of this study, the $\mathrm{OBC}$ has three attributes which contribute to its efficacy as a novel flotation device, viz. 
$\checkmark$ It is able to produce good plug flow conditions relative to a standard flotation column. The benefit of this improved plug flow behaviour is however only significant in high recovery systems.

$\checkmark \quad$ It has a more evenly distributed shear rate than a conventional stirred system, in which particles are exposed to a narrower the range of shear rates. This narrow band of energy dissipation rates can be more efficiently optimised than the broader band occurring in conventional stirred systems.

$\checkmark$ The OBC also produces an additional 'oscillatory component' of fluctuating velocity which is believed to contribute to the particle and bubble fluctuating velocity in the cell. It is proposed that this oscillatory fluctuating velocity allows the $\mathrm{OBC}$ to produce considerably larger r.m.s. fluctuating velocities at a fraction of the power input required in a conventional cell. This increased 'apparent' fluctuating velocity in the cell is thought to improve the flotation kinetics at low power dissipation, but cause significant detachment at moderate power dissipation.

It is due to these factors that the $\mathrm{OBC}$ is able to produce substantial increases in the recovery of all size fractions, particularly for fine particles where the $\mathrm{OBC}$ was able to produce increases of up to $60 \%$ in the flotation rate of sub 36 micron particles under moderately hydrophobic conditions.

As a research tool for evaluating the role which hydrodynamics play in promoting particle-bubble contacting, the kinetics in the OBC have been shown to increase at a decreasing rate with power dissipation before detachment starts to dominate. This is in agreement with the findings of previous experimental studies and is similar to the trends predicted by the fundamental flotation models. Its homogenous energy distribution, variability of power input and the ability to decouple bubble generation from the power input, make it relatively easy to extract trends in terms of power dissipation and particle-bubble contacting for comparison with model predictions. However, the 
anisotropy of the flow in the OBC does mark a distinct difference between this system and the conditions for which flotation fundamental models are derived. Furthermore the additional 'oscillatory component' of the fluctuating velocity is thought to substantially increase rate constants despite the low overall power dissipation in the fluid. Such additional factors mean that the apparent level of power dissipation required to produce contacting is distorted from the value which would be obtained in a more idealised environment.

The effect of the r.m.s. fluctuating velocity on the flotation rate is not taken into account in the shear mechanism models such as Camp \& Stein (1943) and Saffman \& Turner (1956), where only the shear due to the viscous dissipation sub-range eddies in accounted for. The Abrahamson model for accelerative collision predicts a linear relationship between the collision rate and the r.m.s. fluctuating velocity (c.f. Equation 2.41) but only applies to the inertial sub-range of turbulence which would not form at these low power dissipations. It is therefore believed that this test work has exposed an additional mechanism of particle-bubble contacting which is not accounted for in the current range of models. This highlights the importance of bulk flow characteristics in determining flotation kinetics and suggests that a model based on the total shear rate in the system may be more appropriate for this type of hydrodynamic system.

Thus despite the differences between the $\mathrm{OBC}$ and conventional stirred systems, the system has provided some useful insights into the relationship between hydrodynamics and particle-bubble contacting which constitute significant steps forward in understanding this complex behaviour. Using the OBC, it was demonstrated that increases in the power dissipation in the fluid can indeed produce substantial increases in the flotation rate relative to quiescent systems. Power dissipation cannot however be increased indefinitely since detachment of particles of just $3-17$ microns could be observed for power inputs of greater than $50 \mathrm{~W} / \mathrm{m}^{3}$. This strongly supports the hypothesis that the optimal conditions for particle-bubble contacting are indeed significantly lower than those required to produce small bubbles, as was speculated by previous researchers (Deglon, 1998). In addition, the dependency of the flotation rate on the number of bubbles per unit volume was shown to be highly implausible, given that 
the flotation rates were largely unaffected by a 3.7 fold increase in gas hold-up. This strongly indicates that the volumetric basis on which fundamental models are based may require revision since it does not take into account the mean upward velocity of bubbles through the cell.

The efficacy of the $\mathrm{OBC}$ as a research tool therefore has merit as its use has provided some specific insights into the modelling of flotation sub-processes. This can inform the further development of fundamental flotation models and provide a basis for comparison with other studies. The efficacy of the cell as a novel flotation device also seems to have much merit given the consistent and significant improvements in the flotation rate constant, particularly for fine particles.

The following chapter will test the OBC's efficacy in treating a highly rheologically complex nickel ore slimes slurry which is known for its fine grind size and low recoveries due to hydrodynamic difficulties. The information derived will not only inform the OBC's potential for engineering application but also provides an opportunity to vary the micro-turbulent environment in the cell by varying the fluid viscosity. This provides a useful means of assessing the extent to which the kinetics are controlled by either the micro-turbulence in the fluid or by the bulk flow conditions in the cell. 


\section{Chapter 6}

\section{Nickel Ore Slimes Flotation}

In Chapter 5 it was shown that the $\mathrm{OBC}$ is able to produce enhanced flotation kinetics relative to a standard column cell due to its good mixing characteristics and even distribution of shear rate in the system. The additional fluctuating velocity produced by oscillatory flow is also believed to substantially improve the flotation kinetics at low overall power dissipation and increase the detachment rate at moderate power dissipation. The next step in evaluating the efficacy of the $\mathrm{OBC}$ as a novel cell was to assess the cell's performance in an industrial application. Due to its even distribution of energy dissipation rate and the considerable control of the power input to the system, it was hypothesised that the $\mathrm{OBC}$ would be best suited to a niche application in the treatment of fine and rheologically complex ore types which are difficult to treat using conventional cell technologies. The ore selected for testing was a nickel sulphide ore slimes stream from a Western Australian nickel operation on the Agnew-Wiluna Greenstone Belt. This stream is renowned for its fine grind size and complex rheology. The stream is known to contain high mass fractions of fibrous gangue material, mostly Chrysotile, which considerably increases the slurry's yield stress and viscosity, severely complicating cell hydrodynamics in conventional stirred systems.

The testing of the OBC on a highly viscous ore type had the added benefit of allowing an investigation of the effect of rheology on the flotation kinetics, which was shown in 
Chapter 2 to be an important parameter in determining the particle-bubble contacting rate. Increasing the viscosity of the slurry has been shown to produce damping of the micro-turbulent environment (Schubert, 1999) and decreases the shear rate obtained within the viscous dissipation sub-range eddies. Chapter 2 showed that for a shear mechanism of collision, the flotation rate is proportional to the viscosity to the power of $1 / 3$ (c.f. Equation 2.41) and proportional to viscosity to the power of $1 / 2$ for the accelerative mechanism (c.f. Equation 2.42 and 2.47). Rheology does not however affect the bulk fluid motion in an $\mathrm{OBC}$ for $\mathrm{Re}_{\mathrm{o}}>1000$ (Fitch et. al., 2005). Thus altering the rheology of the slurry provides a useful means of assessing whether the kinetics in the cell are indeed controlled by micro-turbulence, as predicted by flotation theory, or whether the effect of bulk hydrodynamics is more significant in determining kinetics in the $\mathrm{OBC}$, as speculated in Chapter 5.

The following chapter details the results of the testing of the $\mathrm{OBC}$ on nickel ore slimes with specific focus on the rheological characteristics of the ore slurry and the OBC performance as a function of power dissipation in the cell. The performance is evaluated at both high and low percent solids in order to manipulate the slurry rheology and evaluate the relative importance of bulk hydrodynamics in determining flotation kinetics in the $\mathrm{OBC}$.

\subsection{Slimes Rheology vs. Percent Solids}

Although slurry rheology can be easily manipulated using chemical agents such as dispersants, the aim of the study was to evaluate OBC performance whilst maintaining constant solution chemistry. For this reason, the percent solids provided the most appropriate means of altering the slurry rheology. This is because the ethyl xanthate collector used in this slurry chemisorbs to the mineral surface and is therefore not affected by changes in the bulk collector concentration. The $\mathrm{pH}$ of the slurry was found to be well buffered in the vicinity of 9.0 and no frother was added prior to flotation since the operation's process water contains an exceptionally high concentration of 
dissolved salts ${ }^{*}$. Variation of the percent solids therefore provides a useful means of altering the slurry rheology without altering particle hydrophobicity or slurry frothing properties. The following section details the nature of nickel ore slimes rheology as a function of the percent solids in the stream.

Slimes rheology was found to exhibit Bingham plastic behaviour with a large yield stress associated with it at high percent solids. An example of the rheogram obtained is given in the following figure:

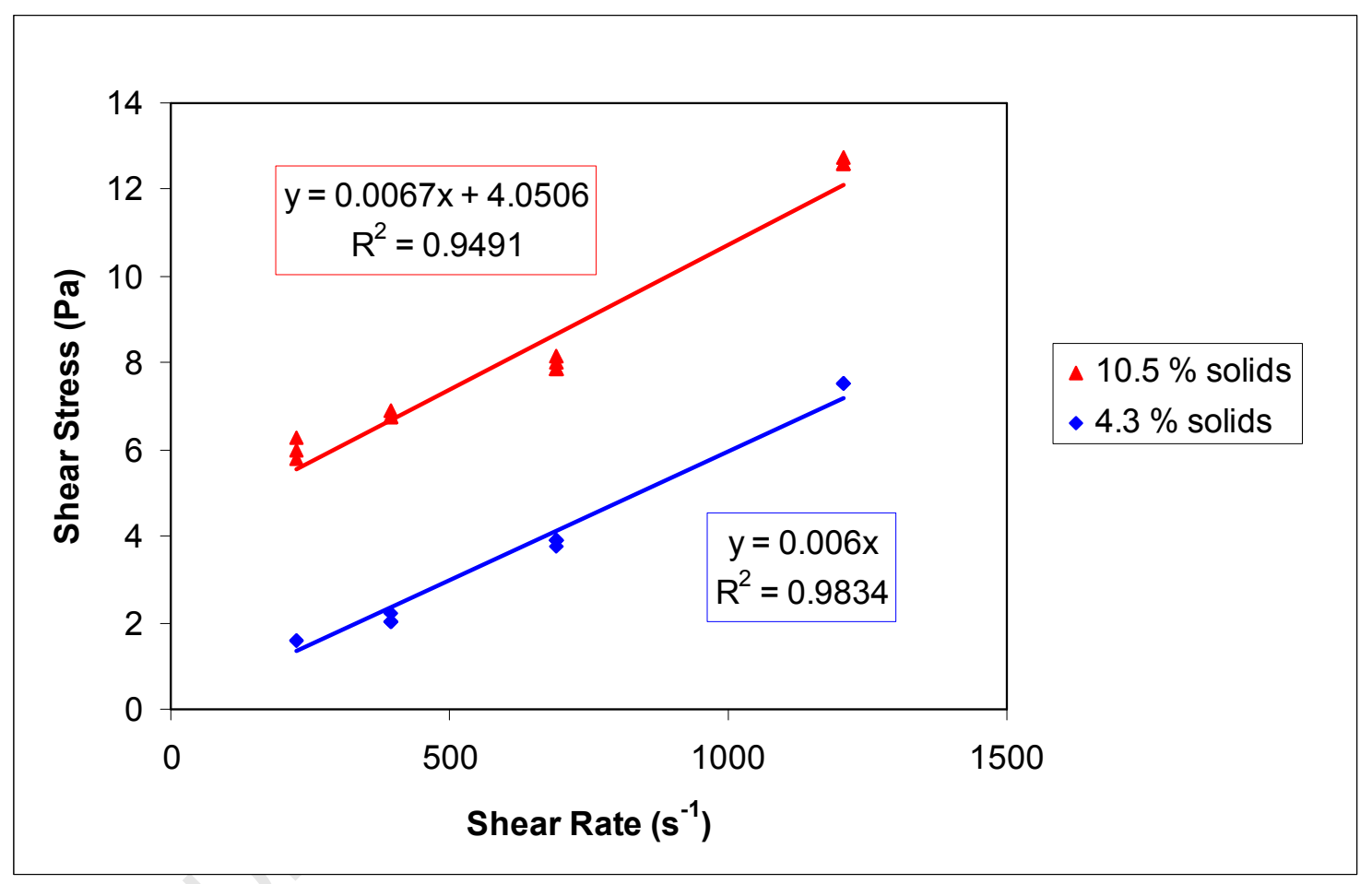

Figure 6.1 - Example of Rheogram obtained for Nickel Ore Slimes at High and Low Percent Solids

As the slurry is diluted, the yield stress drops significantly and slurry starts to exhibit Newtonian behaviour. Interestingly, the Bingham viscosity of the slurry is largely unaffected by variation in percent solids and changes by only $10 \%$ when the percent

\footnotetext{
* The plant process water typically has a TDS of approximately $70000 \mathrm{ppm}$.
} 
solids is decreased from $10.5 \%$ to $4.3 \%$. The effect of percent solids on yield stress and viscosity is summarised in the Figure 6.2:

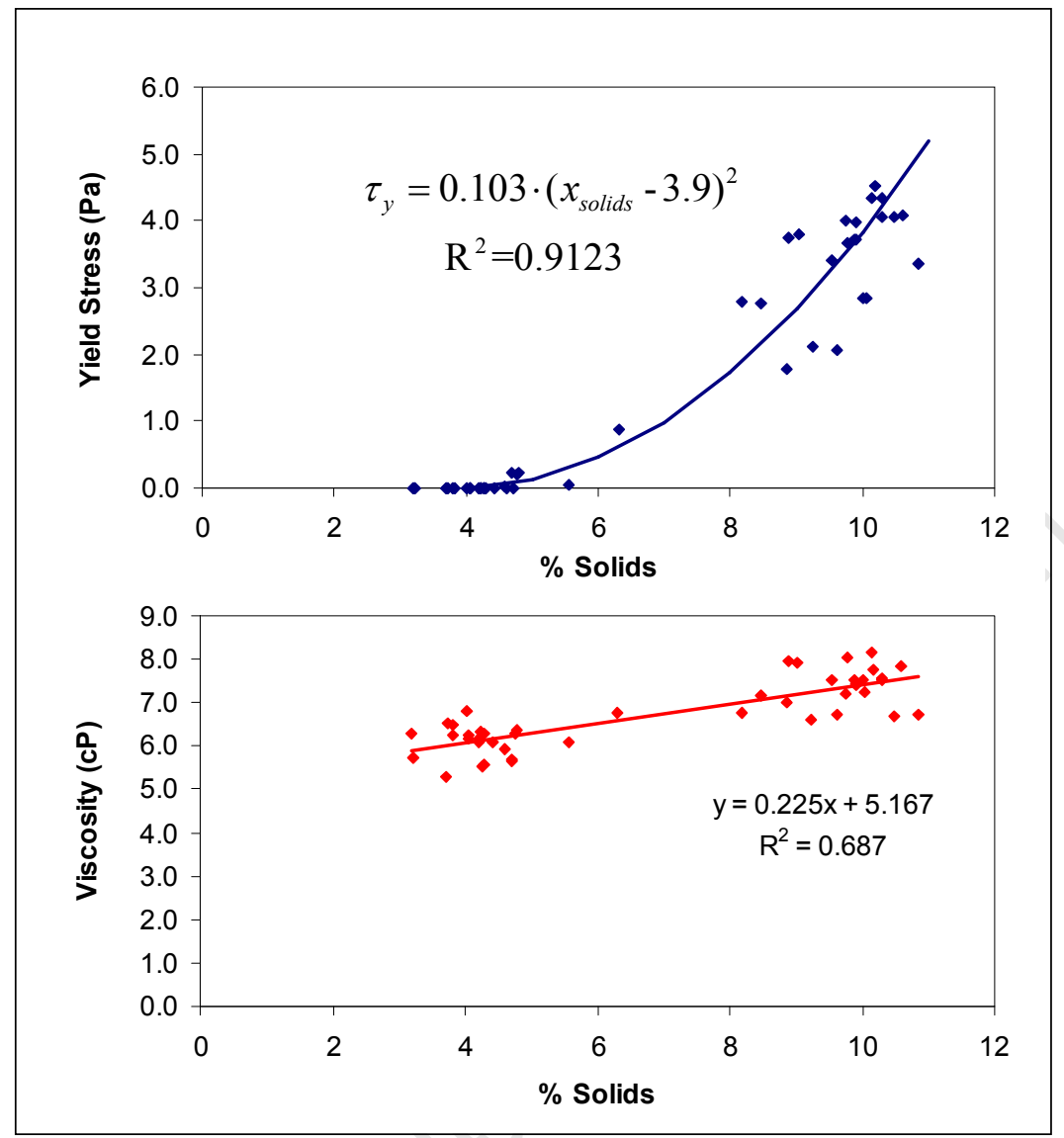

Figure 6.2 - The Effect of Solids Fraction on Yield Stress and Viscosity

Figure 6.2 shows that yield stress follows an exponential relationship with solids fraction. This relationship has been noted by numerous other researchers (Johnson et. al., 2000, Hea et. al., 2004). The scatter about this trend is due in part to the high errors associated with the yield stress calculation, with the standard error in the yield stress being $19 \%$. The scatter may also be due to slight changes in mineralogy and pulp chemistry between tests.

Thus it can be seen that the dominant effect of varying the percent solids is the effect on the yield stress of the slurry. The viscosity is largely unaffected by comparison and changes by approximately $1.5 \mathrm{cP}$ between the high and low percent solids tests. Thus 
the comparison of 2 slurries with solids fractions of $10-12 \%$ solids and $3-4.5 \%$ solids respectively, represents a comparison of a Newtonian and non-Newtonian slurry with similar Bingham viscosities.

\subsection{The Effect of Power Dissipation on the Rate of Flotation}

Figures 6.3 and 6.4 summarise the effect of power dissipation on the flotation rate of each element in the nickel ore slimes stream, with the entrainment contribution removed using the method described in section 3.2.3.5. The point at zero power input denotes the average nickel rate constants obtained using a standard flotation column over 12 different feed tanks, with the error bars indicating the overall range of column rate constants obtained. The other points denote the trend obtained when varying power dissipation while processing feed from a single feed tank. Results are normalised relative to the average rate constant obtained in a standard column cell.

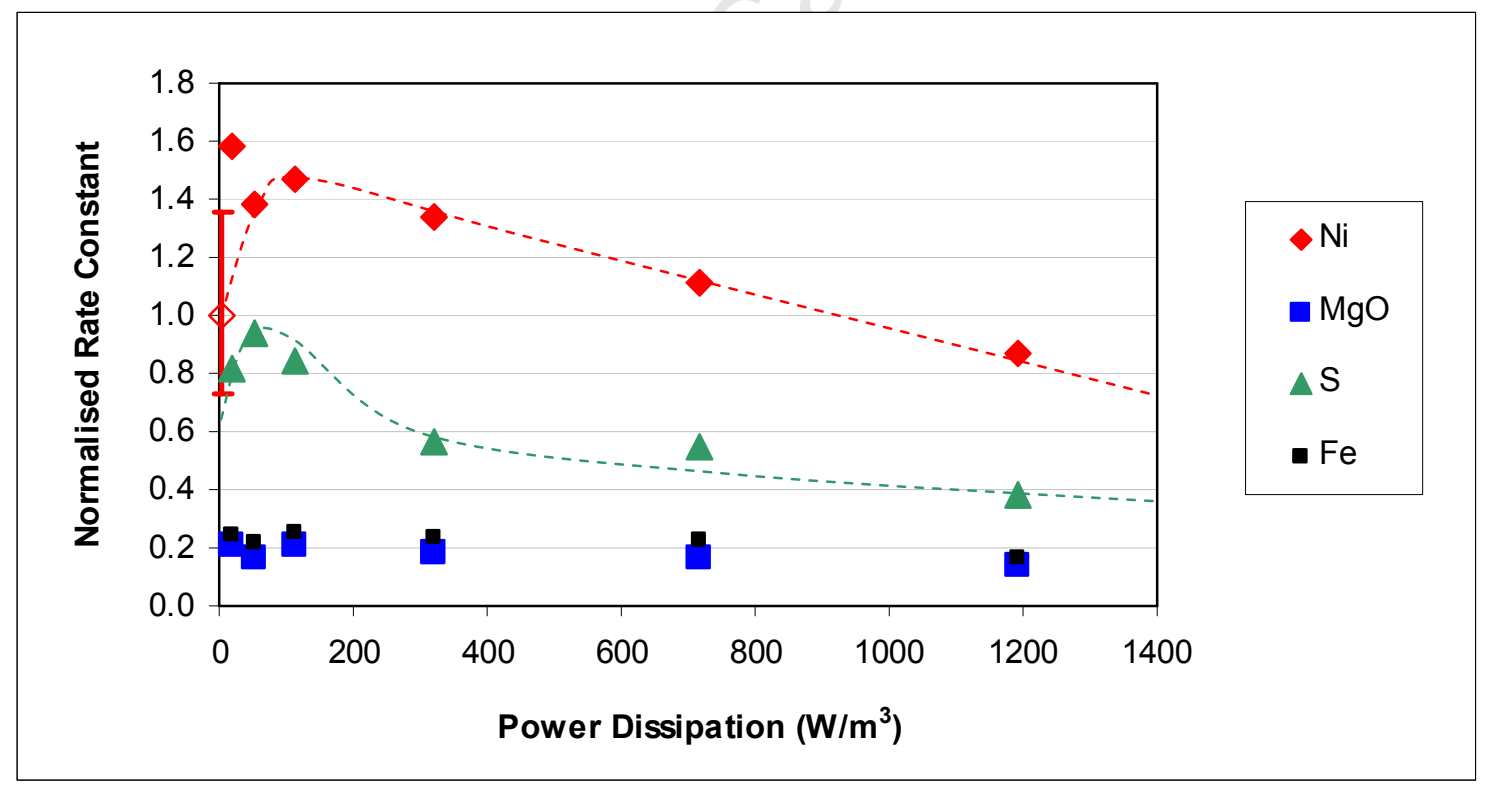

Figure 6.3 - The Effect of Power Dissipation on the Flotation Rate Constant at $11.2 \%$ Solids 


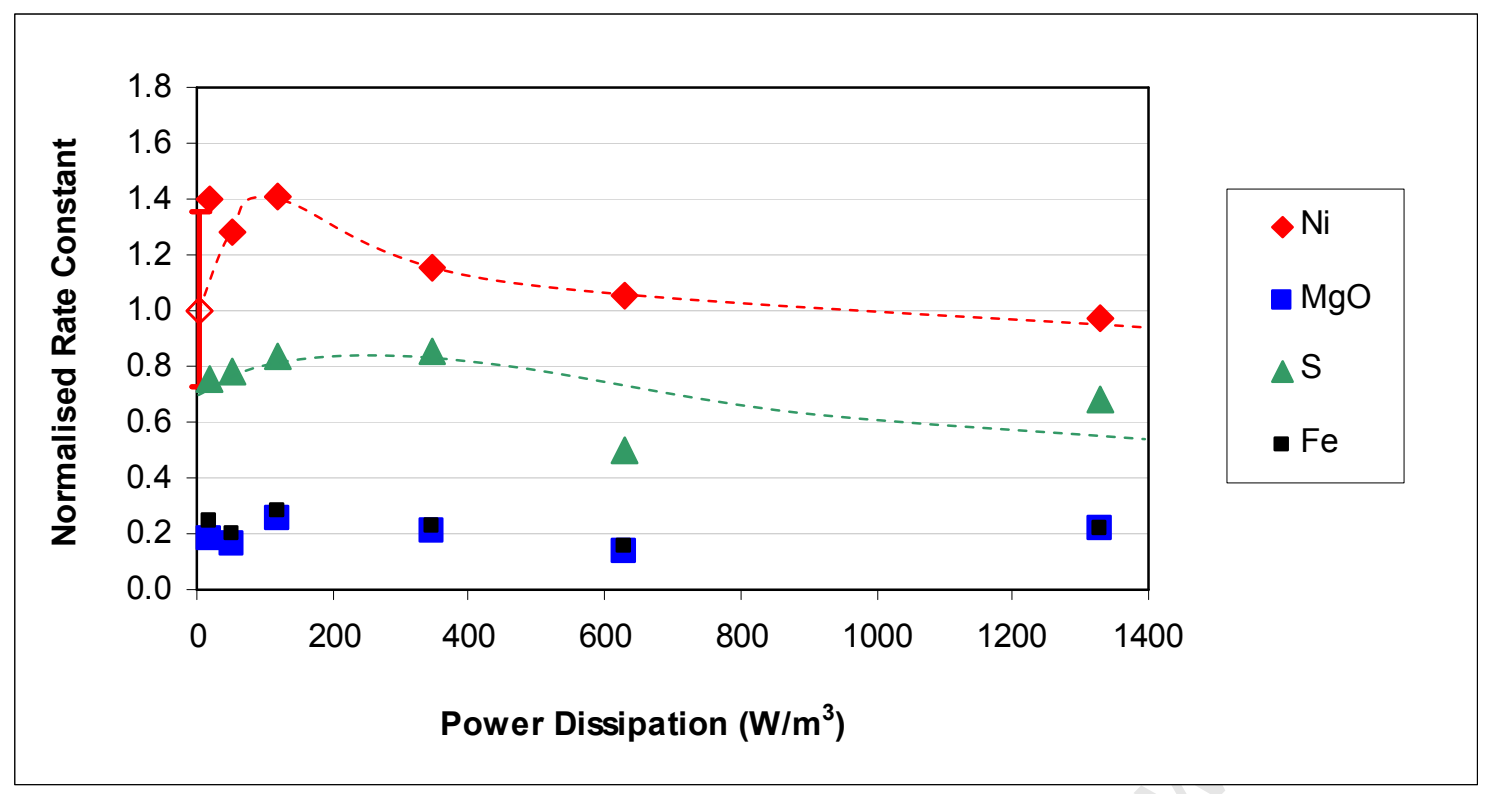

Figure 6.4 - The Effect of Power Dissipation on the Flotation Rate Constant at $4.6 \%$ Solids

Figures 6.3 and 6.4 both exhibit a similar trend with power input. Flotation rate is initially high at $20 \mathrm{~W} / \mathrm{m}^{3}$ and then decreases at $50 \mathrm{~W} / \mathrm{m}^{3}$. The rate constant increases to a maximum at $100 \mathrm{~W} / \mathrm{m}^{3}$ before slowly declining as the power input is further increased.

The results are in good agreement with the results obtained during quartz flotation and, as anticipated, the addition of power input to the system does produce an improvement in the flotation kinetics. The observed rate constants increase to above the range of rate constants observed in a standard flotation column for power inputs between 20 and 350 $\mathrm{W} / \mathrm{m}^{3}$ in both the high and low percent solids tests. Although the standard column tests were conducted on different feed material, they do provide strong circumstantial evidence that the overall rate of flotation is improved by power inputs between 20 and $350 \mathrm{~W} / \mathrm{m}^{3}$ relative to a standard column, as was obtained during quartz flotation. The mixing characteristics of the $\mathrm{OBC}$ are not likely to have a significant impact on the overall kinetics since the overall recovery by true flotation during testing was less than $50 \%$ for all conditions tested. This is consistent with the relatively low increase in kinetics with added energy input, where rate constants are improved by a maximum 
factor of 1.4 to 1.6 by energy addition. This is very similar to the range of the improvements observed in quartz flotation using the tanks-in-series model.

The trends in Figure 6.3 and 6.4 appear to be largely in agreement with flotation theory except for the initially high rate constant obtained at $20 \mathrm{~W} / \mathrm{m}^{3}$. The reason for this unexpectedly high flotation rate at $20 \mathrm{~W} / \mathrm{m}^{3}$ is unclear but it is unlikely to be due to experimental error as the point is reproducible in both the high and low percent solids tests. Furthermore these points were not resolved when the samples were sent for reassay. This point did however coincide with an observed entrapment of bubbles downstream of the oscillating baffles and may in fact be evidence of possible vortex trapping effects in the cell as observed by Mackley et. al. (1993), Baird et. al. (1996) and Levesley \& Bellhouse (1997).

As shown in section 2.2.4, the vortices generated behind the baffles in an $\mathrm{OBC}$ are able to trap bubbles within them by virtue of their centrifugal motion (Tooby et. al., 1977, Baird et al., 1996). These vortices have also been shown to be the regions of highest shear in the cell (Ni et. al., 2002, Bakker, 2005). Entrapment of bubbles in these vortices would therefore result an abnormally high concentration of bubbles in these zones of high shear in the cell, thus leading to higher flotation rates being observed under these conditions. This effect is also more likely to be more prominent in high viscosity systems, such as nickel ore slimes, where the drag forces on the bubbles are more significant and bubble rise velocity is greatly diminished. At higher power inputs however, the flow becomes less structured and more chaotic in nature. Vortices therefore tend to become more distorted and less able to entrap bubbles. A decrease in bubble entrapment may therefore have resulted in the decrease in kinetics observed at $50 \mathrm{~W} / \mathrm{m}^{3}$. It is therefore speculated that this point of higher flotation rate at $20 \mathrm{~W} / \mathrm{m}^{3}$ is a consequence of the $\mathrm{OBC}$ system operating at high viscosity rather than a generic outcome in terms of power input vs. rate constant.

To further discern the validity of this point in terms of particle-bubble contacting, the nickel rate constants were compared with those quartz particles of the same size distribution (0-12 micron). This comparison is illustrated in Figure 6.5 where the rate 
constants are normalised relative to the rate constant at $100 \mathrm{~W} / \mathrm{m}^{3}$ to provide a basis for comparison.

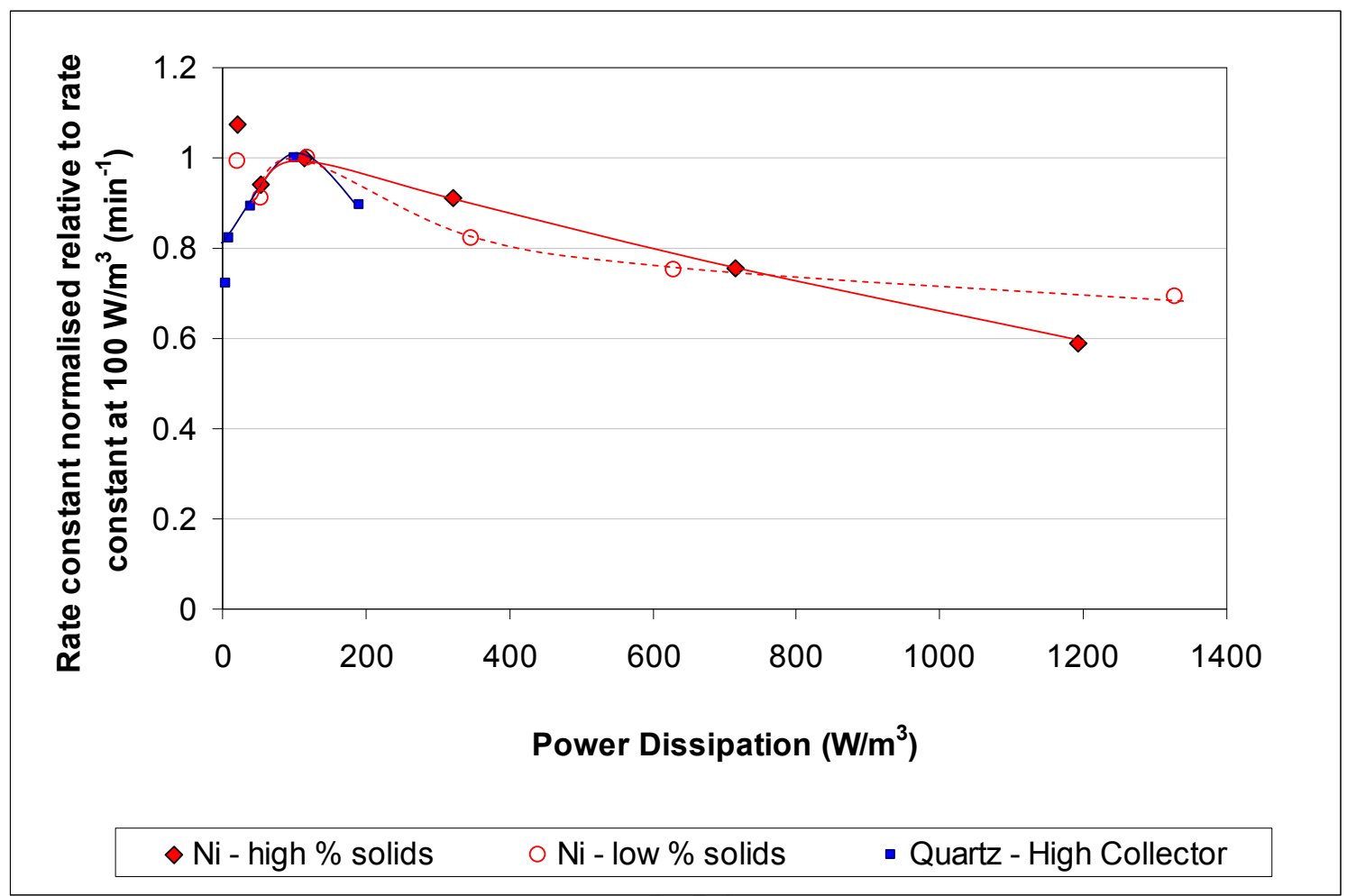

Figure 6.5 - Comparison of rates constants obtained for nickel in MKO slimes flotation and 0-12 micron quartz particles in a quartz-amine system

Figure 6.5 illustrates the striking similarity between the results obtained for quartz and nickel in distinctly different rheological systems. This gives further evidence that the point at $20 \mathrm{~W} / \mathrm{m}^{3}$ is anomalous in terms of the overall energy vs. rate constant trend and is most likely characteristic of the $\mathrm{OBC}$ operating at high viscosity.

Interesting to note is that the trend is very similar despite the large changes in rheology between the different tests. For quartz, the system was very dilute ( $1 \%$ solids) and the slurry was largely Newtonian with a viscosity approximately equal to that of water (1 cP). For nickel at low percent solids, the slurry was Newtonian with a viscosity of 6.3 $\mathrm{cP}$ and for nickel at high percent solids the slurry was non-Newtonian with a yield stress of $5.4 \mathrm{~Pa}$ and viscosity of $7.7 \mathrm{cP}$. This constitutes a substantial change in rheological 
conditions as shown by the significant differences in the apparent viscosity of each slurry as shown in Figure 6.6:

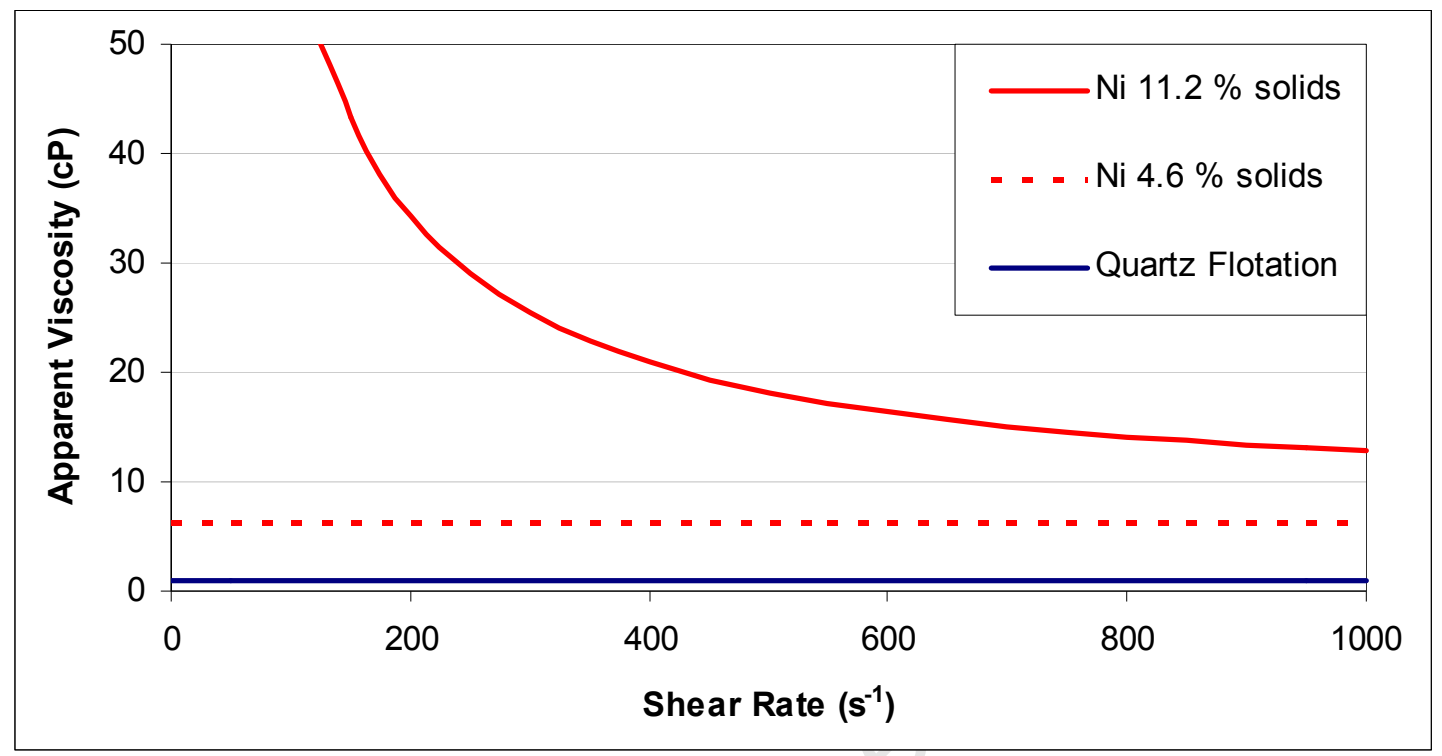

Figure 6.6 - Comparison of Apparent Viscosities of the Different Slurries Tested

Figure 6.6 illustrates the vast changes in apparent viscosity that occur as a function of percent solids between the flotation test conditions. Such an increase in apparent viscosity would have caused substantial changes in the behaviour of the micro-turbulent environment in the fluid. If one follows the approach of Camp \& Stein (1943), the mean velocity gradient in the viscous dissipation sub-range of eddies can be approximated by the inverse of the Kolmogorov timescale of turbulence, viz.

$$
\tau_{k}=\left(\frac{v}{\varepsilon}\right)^{1 / 2}
$$

According to this scale, a six fold increase in the viscosity would require a six fold increase in power dissipation in order to maintain the same mean velocity gradient within the viscous eddies and therefore the same contacting rate. However, the flotation data in Figure 6.5 clearly shows that despite these substantial changes in slurry rheology, very little change in the energy dependence of the flotation kinetics was 
obtained. This strongly suggests that slurry rheology in fact has little effect on particlebubble contacting in the $\mathrm{OBC}$ in this range of viscosity and yield stress. This result is highly unexpected and contrary to the predictions of the flotation fundamental models detailed in Section 2.3.

This independence of flotation kinetics on the rheology of the system strongly suggests that particle-bubble contacting in the $\mathrm{OBC}$ is not driven by the inertial and viscous dissipation sub-range eddies in the fluid as suggested by fundamental flotation models, but rather by another factor which is independent of slurry rheology.

In Chapter 5 it was speculated that the additional fluctuating velocity in an $\mathrm{OBC}$ due to oscillatory flow may in fact have a significant effect on the particle-bubble contacting rate and thus enable the $\mathrm{OBC}$ to produce high collision and detachment rates at exceptionally low power inputs. As noted in Section 2.2.4, Fitch et. al. (2005) investigated the effect of rheology on the flow patterns in an OBC using both CFD and DPIV. Their findings indicated that viscosity and yield stress had little effect on flow patterns in an $\mathrm{OBC}$ for settings where the oscillatory Reynolds number was greater than 1000. In the current study, the oscillatory Reynolds number was maintained at greater than 2100 for all tests conducted and thus the flow pattern in the OBC is likely to have remained unchanged despite the significant changes in slurry rheology. Since the flow pattern and oscillatory velocity in the $\mathrm{OBC}$ are constant with rheology, the fluctuating velocity experienced by particles and bubbles in the fluid is likely to have remained the same. In contrast, the velocity gradients within the viscous dissipation sub-range eddies would have varied greatly in accordance with turbulence theory.

Thus the findings of these tests strongly suggest that the most significant driver of particle-bubble contacting in the OBC was not the velocity gradients within the viscous dissipation sub-range eddies, but rather the velocity gradients due to oscillatory motion of the fluid and the resulting flow patterns which remained unchanged as the slurry rheology was manipulated. This effect would further explain the large particle-bubble collision and detachment rates observed at power inputs an order of magnitude lower than those found in most Rushton turbine cells, for both the quartz and nickel ore slimes 
flotation tests. Furthermore a shear-type collision mechanism driven by the overall shear rate in the fluid may explain the similar trends obtained in terms of power dissipation in the $\mathrm{OBC}$, despite the vast changes in rheology. This overall shear rate in the system is speculated to be dominated by the oscillatory fluctuating velocity which generates additional velocity gradients in the fluid.

It is speculated that the independence of flotation rate on the yield stress and viscosity is therefore unlikely to be found in conventional cells since these cells do not have an oscillatory component of flow, which increases the shear rate due to large scale flow patterns. Based on the findings of previous studies, the slurry yield stress can have a significant impact of the shear rate distribution and gas dispersion in the cell (Deglon et. al., 2007). Furthermore, large yield stresses have been shown to lead to cavern formation in stirred systems which can greatly diminish the agitated volume of the cell (Moore \& Cossor, 1995, Fangary et. al., 2000, Arratia et. al., 2006). According to turbulence theory, increased viscosity increases the mean eddy size in the cell and therefore decreases the mean shear rate which particles and bubbles are exposed to. This in turn leads to decreased collision and detachment rates at increased viscosity. This would mean that more power must be input to achieve the same contacting rate at a higher fluid viscosity. Thus it is thought to be unlikely that such a similar trend would arise in a conventional cell when the rheology is varied over such a wide margin.

This finding is very significant and has implications both for the application of the OBC as a novel cell and the use of the $\mathrm{OBC}$ as research tool in which to evaluate particlebubble contacting, as it marks a distinct difference between this device and conventional flotation machines.

This independence of flotation kinetics on slurry rheology gives the $\mathrm{OBC}$ a distinct advantage in the treatment of rheologically complex material. The cell is able to produce a 1.4 to 1.6 fold increase in kinetics relative to a standard column, irrespective of the rheological conditions, since it is not affected by the detrimental effects of rheology on cell hydrodynamics and gas dispersion. 
As a research tool, the OBC does provide a useful means of decoupling gas dispersion and solid suspension effects from the effect of energy on particle-bubble contacting. In addition, the even distribution of shear rate in the cell provides a more appropriate environment for testing the trends of fundamental models which are based on a homogeneous distribution of localised turbulence variables, such as the energy dissipation rate and the mean squared fluctuating velocity. Ultimately however, the driver of kinetics in an $\mathrm{OBC}$ is unlikely to be the small scale turbulent eddies for which the fundamental models are derived, but rather is driven by the shear rate due to the bulk fluid motion associated with oscillatory flow. This oscillatory component of fluid flow means that shear rate per unit power input to the cell is considerably larger than both homogeneous isotropic turbulence and the turbulence found in conventional flotation machines. Optimal flotation conditions are therefore obtained at substantially lower power inputs than in more idealised systems. 


\section{Chapter 7}

\section{Summary \& Conclusions}

This thesis has presented an evaluation of an Oscillatory Baffled Column as a novel flotation device and as a research tool for investigating the role which hydrodynamics play in promoting particle-bubble contacting. The cell differs from a conventional cell design in that bubble generation in the cell is decoupled from the power input, allowing the hydrodynamic environment to be optimised for effective particle-bubble contacting. The design also incorporates a novel mechanism of agitation which provides a more even distribution of shear rate than a stirred system, and allows considerably more variation of the power input to the cell. Based on these properties it was hypothesised that the $\mathrm{OBC}$ would provide an effective hydrodynamic environment for fine particle flotation, particularly in highly viscous, non-Newtonian slurries. It was also hypothesised that the development of such a cell technology would facilitate an improved understanding of the role which hydrodynamics play in promoting particlebubble contacting.

In order to investigate these hypotheses, the OBC was first pre-characterised in terms of its hydrodynamic behaviour. The cell was then flotation tested on quartz so as to benchmark the study against previous studies in stirred systems. The study was then extended to the flotation of a highly viscous, non-Newtonian nickel ore slimes slurry 
which is difficult to treat using conventional cell technologies. This study gave context to the potential future role of $\mathrm{OBC}$ technology in the minerals processing industry.

The results of this thesis have provided numerous insights into both the operating characteristics of OBC's as well as the underlying drivers of particle-bubble contacting in this unique hydrodynamic environment. The following chapter summarises the key findings of the study, before discussing their implications for the application of the $\mathrm{OBC}$, both as a novel flotation device and as a research tool for investigating particlebubble contacting. Conclusions are then drawn and recommendations are made for future work.

\subsection{Summary of Key Findings}

\subsubsection{Hydrodynamic characteristics of the OBC}

The hydrodynamic characteristics of the OBC were found to be significantly different from those of a conventional stirred system, in that the fluid motion in the cell oscillates with time. This additional oscillatory motion of fluid results in a greater 'apparent' root mean squared fluctuating velocity per unit power input than would be obtained in a nonoscillating fluid environment.

The oscillatory nature of flow in the $\mathrm{OBC}$ also means that the power characteristics of the cell are oscillatory. Power input is therefore characterised by the time-averaged power dissipation in the cell. The power characteristics were found to be in good agreement with models from the literature, although these models were unable to predict the power behaviour under high gas hold-up conditions. The measured values of power dissipation were therefore used under these conditions. The modelling of power characteristics at high gas hold-up is highly complex and new models are required to describe this behaviour. 


\subsubsection{The Effect of Power Dissipation on Gas Phase Characteristics in the OBC}

Bubble size was shown to remain relatively constant with power dissipation over the range of power dissipations tested. A small deviation was noted at low power dissipation, however this deviation was found to have an insignificant effect on the kinetics relative to the large effect of power dissipation. The assumption of a constant bubble size in the $\mathrm{OBC}$ was therefore considered to be valid.

The gas hold-up, in contrast, was found to increase significantly with increased power dissipation during the quartz flotation tests due to the increased reversing flow under oscillatory flow conditions. According to the framework of the fundamental flotation models (c.f. equation 2.32), the flotation rate is directly proportion to the number of bubbles per unit volume and therefore to the gas hold-up in the system. In the current study, gas hold-up increased 3.7 fold over the range of power inputs tested, but the bubble surface area flux through the system was maintained constant. According to the fundamental models of flotation, this 3.7 fold increase in gas hold-up would have produced a 3.7 fold increase in the rate constant over the same range of power inputs. The results of the quartz flotation tests however clearly indicate that no such dependence is present since rate constants were only improved by 1.6 fold over the range of conditions tested. This would suggest that the framework for the development of fundamental flotation models needs to be reviewed in terms of its gas hold-up dependence to rather incorporate a flux based approach.

\subsubsection{Mixing Characteristics of the OBC}

The OBC was found to produce enhanced plug flow conditions relative to a standard flotation column for all power inputs less than $200 \mathrm{~W} / \mathrm{m}^{3}$. This improved plug flow behaviour was however shown to only have a significant effect on the overall flotation kinetics if the recovery of material was high (i.e. $\mathrm{R}>70 \%$ ). For this reason, mixing was taken into account when evaluating the results of the moderately hydrophobic flotation tests. However, the effect of mixing on the nickel ore slimes flotation rate was considered to be negligible, since the overall recoveries during flotation testing were less than $50 \%$ for all conditions tested. The improved mixing characteristics of the 
OBC therefore do not constitute a strong motivation for its potential industrial application as a novel cell, since the recovery obtained in most industrial applications is likely to be considerably lower than those obtained during quartz flotation.

\subsubsection{Flotation Performance in an OBC}

The $\mathrm{OBC}$ was found to produce significant increases in the flotation rate constant relative to a standard column for both quartz and nickel ore slimes. For quartz flotation, the flotation rate was significantly improved in all size fractions, particularly for fine particles where the tanks-in-series rate constant was improved by a factor of 1.4 to 1.6 relative to a standard column. Interestingly, this increase was also found to be independent of slurry rheology, since a similar increase of 1.4 was also demonstrated on the nickel slimes slurry despite the greatly increased yield stress and viscosity associated with the slurry. This indicates that the OBC was indeed effective in the treatment of this fine and rheologically complex ore type.

\subsubsection{The Effect of Power Dissipation on the Flotation Rate}

The flotation rates obtained in the $\mathrm{OBC}$ were in reasonable agreement with the theoretically expected trends in terms of particle size, particle hydrophobicity and power dissipation. As expected, the flotation rate was found to increase with increasing particle size from 3 to 104 microns. A significant improvement in the flotation rate was obtained when power was added to the system for both quartz and nickel ore slimes. Quartz flotation rate constants were found to increase with added power for power dissipations of less than $50 \mathrm{~W} / \mathrm{m}^{3}$, before an increased rate of detachment caused the flotation rate of fine particles to level off and the flotation rate of coarser particles to gradually decrease. This gave an indication of fine particle detachment in the system which is contrary to the widespread belief that fine particles do not detach. The point of decrease in flotation recovery was found to be a strong function of particle hydrophobicity and increased by nearly an order of magnitude input from $10 \mathrm{~W} / \mathrm{m}^{3}$ to between 40 and $100 \mathrm{~W} / \mathrm{m}^{3}$, between the two particle hydrophobicity settings tested. 
Interestingly, the amplitude of power input was found to have little effect on the flotation kinetics beyond improving the mixing conditions. The distribution of shear in the system was therefore assumed to be similar for all amplitudes.

\subsubsection{Comparison of Flotation Trends with Previous Studies}

The trends obtained in terms of power input were found to be in good agreement with the findings of studies conducted in Rushton turbine flotation cells (Deglon, 1998, Pyke, 2004, Newell, 2006). However the optimum power conditions observed in the OBC were found be an order of magnitude lower than those in Rushton turbine systems. This discrepancy is attributed to innate differences in the hydrodynamic environment between the $\mathrm{OBC}$ and conventional impeller-driven systems rather than differences in particle hydrophobicity.

In terms of hydrodynamics, the OBC produces a considerably more evenly distributed shear rate than a conventional stirred system. The shear rate is therefore more efficiently distributed so as to maximise the flotation kinetics over a greater volume of the cell than in a stirred system.

Secondly, the oscillatory motion of the fluid in an OBC produces a substantial addition to the 'apparent' mean squared fluctuating velocity of particles and bubbles in the fluid. The findings of this study strongly suggest that this fluctuating velocity due to the oscillatory fluid motion substantially influences the flotation kinetics, and is responsible for the significant increase in both the collision and detachment rates in the cell at power inputs as low as $50 \mathrm{~W} / \mathrm{m}^{3}$.

Furthermore results obtained from flotation of highly viscous nickel ore slimes indicate evidence of vortex trapping at power inputs in the vicinity of $20 \mathrm{~W} / \mathrm{m}^{3}$. This type of effect has also been demonstrated by previous studies on oscillatory flow (Mackley \& Ni, 1993, Baird et. al., 1996, Levesley \& Bellhouse, 1997, Chew et. al., 2004) and may explain the anomalously high rate constants obtained at these particular settings. Vortex trapping would create higher concentrations of particles and bubbles in the regions of 
highest shear in the cell, thereby augmenting contacting rates. Further investigation is required to ascertain the exact conditions under which this effect is significant in improving flotation rates, but the results of this study seem to indicate that low power inputs and high slurry viscosities may be conducive to this phenomenon occurring.

\subsubsection{The Relationship between the Rate of Flotation and Slurry Rheology}

The results of this study have shown that slurry rheology did not have a significant effect on the flotation response of nickel ore slimes as a function of power dissipation. This is contrary to the predictions of the fundamental models which indicate a strong dependence of collision frequency on the viscosity of the fluid.

Previous studies on OBC's however indicate that the large scale eddies and flow patterns generated in an $\mathrm{OBC}$ are in fact independent of rheology for $\mathrm{Re}_{\mathrm{o}}>1000$ (Fitch et. al., 2005). It is therefore believed that contacting in the OBC was largely controlled by the bulk fluid motion in the cell rather than the inertial and viscous dissipation subrange eddies in the fluid. This finding would be consistent with the low overall power inputs at which flotation rates were improved, since the development of turbulent eddies was not necessary to drive kinetics. The OBC therefore operates in an intermediate range of power inputs where kinetics are more influenced by bulk fluid motion than by small scale turbulence.

This is an extremely important finding which has significant implications for the $\mathrm{OBC}$, both as a novel flotation device and as a research tool in which evaluate particle-bubble contacting. 


\subsection{Conclusions}

Based on these findings of this study, the following conclusions can be drawn.

\subsubsection{The Application of the $\mathrm{OBC}$ as a Novel Cell}

The $\mathrm{OBC}$ is concluded to have four major attributes which make it an effective novel flotation device for fine particle recovery, particularly in highly viscous, non-Newtonian slurries, viz.

$\checkmark$ The OBC is able to decouple the effect of power dissipation on bubble generation from its effect on particle-bubble contacting. This means that each sub-process may be independently optimised, allowing for more efficient use of the energy input in the system. Such optimisation is not possible in a conventional flotation cell, where these processes are inextricably interdependent.

$\checkmark$ The OBC produces a more even distribution of shear rate than a conventional cell, in which particles and bubbles are exposed to a narrower range of shear rates. This narrow band of shear can be more efficiently optimised than the broader band occurring in conventional stirred systems. This allows for high contacting rates at considerably lower power inputs.

The OBC also produces an additional 'oscillatory component' of fluctuating velocity due to the oscillatory motion of the fluid. This allows the $\mathrm{OBC}$ to produce a considerably larger root mean squared fluctuating velocity of particles and bubbles in the fluid, at a fraction of the power input required by a conventional cell. This increased fluctuating velocity is believed to substantially to improve the flotation kinetics at low overall power inputs by increasing the mean shear rate to which particles and bubbles are exposed. 
$\checkmark$ Flotation kinetics in the $\mathrm{OBC}$ are independent of slurry rheology since the kinetics are controlled by the bulk fluid motion rather than the micro-turbulent environment. It is therefore believed that $\mathrm{OBC}$ shows considerable potential for application to the treatment of highly viscous, non-Newtonian slurries.

Given these attributes, the $\mathrm{OBC}$ is considered to be an effective and promising novel device. It has been trialled on both a quartz-amine slurry and a highly viscous, nonNewtonian nickel ore slimes stream and has demonstrated its ability to enhance flotation rates by between 1.4 and 1.6 fold relative to a standard flotation column.

The wider application of $\mathrm{OBC}$ technology will however require some further research. The focus of this study has been on the pulp phase performance with froth effects removed from the system. A wider investigation of froth phase performance in the OBC is required to confirm the overall performance achievable. The scale-up behaviour of the device is also identified as a key area for the future development of this technology.

\subsubsection{The Use of the OBC as a Research Tool}

At the outset of this project, it was believed that the development of the $\mathrm{OBC}$ as a novel flotation device would also provide a unique opportunity for the investigation of energy and particle-bubble contacting in an alternative hydrodynamic environment. Such an investigation was intended to highlight possible shortfalls of the current flotation models in describing the role of hydrodynamics in promoting particle-bubble contacting, and assess the relative importance of the mode of power input to the system. This thesis has therefore also investigated the efficacy of using the $\mathrm{OBC}$ as a research tool for this purpose. The trialling of the OBC in this application has achieved some successes and has highlighted some distinct areas for the future development of fundamental flotation models.

As a research tool, the OBC's homogenous energy distribution, variability of power input and the ability to decouple bubble size characteristics from the power input, make it relatively easy to extract trends in terms of power dissipation and particle-bubble 
contacting for comparison with model predictions. The trends obtained have been shown to be similar to those obtained in previous studies, although the optimal power conditions were found to be substantially lower.

The study has also yielded some useful insights into the relationship between hydrodynamics and particle-bubble contacting, which constitute significant steps forward in understanding this complex behaviour. Using the $\mathrm{OBC}$, it has been shown that the particle-bubble contacting rate can be affected not only by the micro-turbulent environment, but by the bulk fluid hydrodynamics as well. This effect is unaccounted for in the current range of fundamental flotation models and may in fact be more important in some flotation systems than previously thought. While the relative importance of micro-turbulence may be greater in a conventional cell since no oscillatory component of fluid flow is present, the particle-bubble contacting rate may still be affected by the shear rate due to bulk fluid motion. It is proposed that in order to accurately predict the flotation behaviour in real flotation systems, the effect of the shear rate due to bulk hydrodynamics on particle-bubble contacting may need to be taken into account.

This study has also provided an interesting opportunity to vary the gas hold-up during flotation without affecting the bubble size or bubble surface area flux through the cell. The results showed that the proposed dependency of the flotation rate of the number of bubbles per unit volume is highly implausible, given that the flotation rates were largely unaffected by a 3.7 fold increase in gas hold-up at constant bubble surface area flux. This strongly suggests a flux based approach to modelling of flotation systems may be more appropriate than the volumetric based approach used by the current range of turbulent flotation models. This volumetric approach does not account for the nett upward flux of bubbles through the system which may be more important than their number density. It is therefore believed that the framework used in deriving fundamental flotation models requires further development and that the possibility of a flux based approach should be considered. 
In terms of cell operating conditions, the study has shown that significant particlebubble detachment can occur even for the finest particle size fractions in the feed at power inputs as low as $50 \mathrm{~W} / \mathrm{m}^{3}$. This behaviour was observed for both quartz and nickel ore slimes and is contrary to the widespread belief that fine particles are insusceptible to detachment forces. This strongly supports the hypothesis that the optimal conditions for particle-bubble contacting are indeed significantly lower than those required to produce small bubbles, as was speculated by previous researchers. This finding, if also true in a conventional stirred system, would have significant implications for cell design, since the methodology of maximising the shear rate at the impeller for bubble generation may be inappropriate for promoting effective particlebubble contacting. Optimal flotation cell design is therefore likely to be a trade-off between the generation of fine bubbles at the impeller and maximising the transmission of energy into the bulk of the cell in order to promote effective contacting rates over a larger volume of the cell.

The $\mathrm{OBC}$ does however have significant limitations in terms of being used as a research tool for the direct validation of the trends predicted by fundamental flotation models. Firstly, the flow in the $\mathrm{OBC}$ is highly anisotropic, and given the low power dissipation at which the $\mathrm{OBC}$ operates, it is doubtful whether the turbulence in the cell would be sufficiently developed for the development of inertial sub-range eddies under most flotation conditions. More significantly, the underlying mechanisms driving particlebubble contacting in an OBC appear to be distinctly different from those derived for homogeneous isotropic turbulence, since the contacting rate in the $\mathrm{OBC}$ is principally controlled by the bulk fluid motion rather than the micro-turbulent environment in the fluid.

Overall however, the $\mathrm{OBC}$ is concluded to be an effective research tool which has provided some useful insights into the effect of hydrodynamics on particle-bubble contacting. Although the system is not ideally suited to validating the trends predicted by fundamental flotation models, the system has been able to highlight areas where our understanding is incomplete and requires further development. This constitutes a 
significant step towards understanding the complex effect of hydrodynamics on particle-bubble contacting in flotation systems.

\subsection{Recommendations for Future Work}

In light of the findings of this thesis, the following recommendations can be made:

\subsubsection{Application of $\mathrm{OBC}$ as a Novel Cell}

This thesis has demonstrated the unique potential of $\mathrm{OBC}$ technology as a means recovering fine material from rheologically complex mineral slurries. It is believed that the increasing prevalence of ultrafine grinding techniques in modern minerals processing operations creates significant scope for the wider application of OBC technology. The scale-up behaviour of OBC's has been well characterised in other applications and can be achieved based on the dimensionless numbers detailed in section 3.1 together with the desired power input to the cell, as calculated from the correlations in section 4.1.1.4. The method of pulsation also presents a number of options based on the various solvent extraction designs as well as the pulsation mechanisms of jigs, which are commonly used in minerals processing applications.

It is therefore recommended that $\mathrm{OBC}$ technology be tested on other industrial mineral slurries, particularly where ultrafine grinding techniques are employed. It is anticipated that such an investigation will further demonstrate the OBC's effectiveness in fine particle flotation and open the door to a wider range of applications than those demonstrated in this thesis.

In order to fully apply $\mathrm{OBC}$ technology industrially, two major areas of future research are identified:

$>$ The mode of oscillation should be investigated to assess whether the design could be altered to include fixed baffles with a pulsed fluid, using a piston or bellows. This alteration in design may considerably simplify many of the engineering difficulties 
associated with internal moving parts which may be encountered on an industrial scale. Oscillation via pulsation of the fluid does however cause oscillation of the level in the column, which may negatively affect froth phase performance and complicate level control systems.

The effect of oscillation on froth phase performance should be investigated, to ascertain whether oscillation has any negative effects on froth recovery or if it results in improved froth drainage. It is speculated that the $\mathrm{OBC}$ will be able to achieve similar deep froth depths to standard flotation columns and will therefore be able to produce a high grade product.

\subsubsection{The Investigation of Hydrodynamics and Particle-Bubble Contacting}

This thesis has shown that the investigation of micro-turbulence and particle-bubble contacting using an $\mathrm{OBC}$ is limited in that the kinetics are dominated by the bulk fluid motion rather than the micro-turbulence in the fluid. In order to study the effect of micro-turbulence on particle-bubble contacting, and thereby validate the trends of fundamental flotation models, it is recommended that a more appropriate device be developed which mitigates any oscillatory motion of the bulk fluid. Such a device is likely to have a similar design to the $\mathrm{OBC}$, with the gas dispersion decoupled from the particle-bubble contacting rate using a micro-bubble generation device, but would incorporate an alternative baffle design which would not cause bulk fluid movement and high fluctuating velocities. One such promising development is the oscillating grid flotation cell (Changunda et. al., 2007), which is similar in design to the OBC but makes use of oscillating grids, rather than orifice baffles, to produce relatively homogeneous and isotropic grid turbulence in the fluid.

The study has also shown that the role of bulk flow characteristics can be significant in determining flotation kinetics. This effect is not taken into account in the current range of fundamental flotation models which ascribe micro-turbulence as the primary driver of contacting. It is therefore recommended that these models be revisited with the view of incorporating the effect of the total shear rate in the fluid rather than only the shear 
rate within the smallest eddies. This may serve to make the models more applicable to real systems where agitation produces large scale flow patterns and shear rates, which augment the shear rate due to micro-turbulence.

Furthermore, it is recommended that a flux based fundamental model framework be established which is better able to predict the dependence of the flotation rate on gas dispersion effects. 


\section{References}

Abrahamson, J., Collision rates of small particles in a vigorously turbulent fluid. Chem. Eng. Sci., 30, 1371-1379 (1975).

Ahmed, N. \& Jameson, G. J., The effect of bubble size on the rate of flotation of fine particles. Int. J. Miner. Process., 14, 195-25 (1985).

Amelunxen, R., The Contact cell: a future generation of flotation machines. Promotional Literature, Amelunxen-Wales Technologies Inc., (Jan. 26, 1993). op. cit. Finch (1995).

Arbiter, N., Development and scale-up of large flotation cells. In: Parekh, B. K. \& Miller, J. D. (Eds.) Advances in Flotation Technology. Littleton, CO, USA, SME (1999).

Arratia, P. E., Kukura, J., Lacombe, J. \& Muzzio, F. J., Mixing of Shear-Thinning Fluids with Yield Stress in Stirred Tanks. AIChE Journal, 52, 2310-2322 (2006).

Baird, M. H. I. \& Garstang, J. H., Power consumption and gas hold-up in a pulsed column. Chem. Eng. Sci., 22, 1663-1673 (1967).

Baird, M. H. I., Rama Rao, N. V. \& Stonestreet, P., Power dissipation and hold-up in a gassed reciprocating baffle-plate column. Trans IChemE, 74, 463-470 (1996).

Baird, M. H. I. \& Stonestreet, P., Energy dissipation in oscillatory flow within a baffled tube. Trans. IChemE, 73, 503-511 (1995).

Bakker, C. W., Numerical Modelling of an Oscillatory Baffled Column. MSc Thesis, University of Cape Town, Department of Chemical Engineering, Cape Town (2005).

Bakker, C. W., Numerical Modelling of non-Newtonian slurry in a Mechanical Flotation Cell. Personal Communication. University of Cape Town (2008). 
Batchelor, G. K., Pressure fluctuations in isotropic turbulence. Proc. Cambridge Phil. Soc., 47 (1951).

Bisshop, J. P. \& White, M. E., Study of particle entrainment in flotation froths. Trans. IMM, 85, C191-C194 (1976).

Bloom, F. \& Heindel, T. J., On the structure of collision and detachment frequencies in flotation models. Chem. Eng. Sci., 57, 2467-2473 (2002).

Boutin, P. \& Wheeler, D. A., Column Flotation. Mining World, 20, 47-50 (1967).

Brady, M. R., Telionis, D. P., Vlachos, P. P. \& Yoon, R. H., Velocities of particles and bubbles in grid turbulence measured by Particle Image Velocimetry. Centenary of Flotation Symposium. Brisbane, Australia, (2005).

Breytenbach, J. N., An investigation of particle collection efficiency in different particle-bubble contacting environments in flotation. MSc Thesis, University of Cape Town, Chemical Engineering, Cape Town (1995).

Brunold, C. R., Dickens, A. W., Mackley, M. R., Williams, H. R. \& Hunns, J. C., Improvements in or relating to mixing apparatus and processes. European Patent Office, WO8700079 (1987).

Brunold, C. R., Hunns, J. C., Mackley, M. R. \& Thompson, J. W., Experimental observations on flow patterns and energy losses for oscillatory flows in ducts with sharp edges. Chem. Eng. Sci., 44, 1227-1244 (1989).

Burdukova, E., Surface Properties of New York Talc as a Function of pH, Polymer Adsorption and Electrolyte Concentration. PhD Thesis, University of Cape Town, Department of Chemical Engineering, (2007).

Camp, T. R. \& Stein, P., Velocity gradients and internal work in fluid motion. J. Boston Soc. Civ. Eng., 30, 219-237 (1943).

Chang, C. \& Powell, R. L., Effect of particle size distributions on the rheology of concentrated bimodal suspensions. J. Rheology, 38, 85-98 (1994).

Changgen, L. \& Bahr, A., Flotation of copper ore in a Pneumatic flotation cell. Minerals Metallurg. Proc., 9, 7-12 (1992).

Changunda, K., Deglon, D. A. \& Harris, M. C., Investigating the effect of energy on flotation kinetics using a novel oscillating grid cell. Flotation '07. Cape Town, MEi (2007). 
Chew, C. M., Ristic, R. I., Reynolds, G. K. \& Ooi, R. C., Characterisation of impeller driven and oscillatory mixing by spatial and temporal shear rate distributions. Chem. Eng. Sci., 59, 1557 - 1568 (2004).

Cowburn, J. A., Stone, R., Bourke, S. \& Hill, B., Design developments of the Jameson cell. Centenary of Flotation Symposium. Brisbane, Australia, AusIMM (2005).

Dai, Z., Dukhin, S. S., Fornasiero, D. \& Ralston, J., The inertial hydrodynamic interaction of particles and rising bubbles with mobile surfaces. J. Colloidal Interface Sci., 217, 70-76 (1998).

Dai, Z., Fornasiero, D. \& Ralston, J., Particle-bubble collision models - a review. $A d v$. Colloid Interface Sci., 85, 231-256 (2000).

Danckwerts, P. V., Continuous-flow systems: Distribution of residence times. . Chem. Eng. Sci., 2, 1-13 (1953).

Davidson, L., An introduction to turbulence models. Publication 97/2, Chalmers University of Technology, Department of Thermo and Fluid Dynamics, Goteborg (2003).

Deglon, D. A., A hydrodynamic investigation of fine particle flotation in a batch flotation cell. PhD Thesis, University of Cape Town, Department of Chemical Engineering, Cape Town (1998).

Deglon, D. A., A novel attachment-detachment kinetic model. In: Ralston, J., Miller, J. D. \& Rubio, J. (Eds.) Flotation and Flocculation: from Fundamentals to Applications. Hawaii, USA (2002).

Deglon, D. A., The effect of agitation on the flotation of platinum ores. Minerals Eng., 18, 839-844 (2005).

Deglon, D. A., Sawyerr, F. \& O'Connor, C. T., A model to relate the flotation rate constant and the bubble surface area flux in mechanical flotation cells. Minerals Eng., 12, 599-608 (1999).

Deglon, D. A., Shabalala, N. Z. P. \& Harris, M. C., Rheological effects on gas dispersion. Flotation 07. Cape Town, MEi (2007).

Degner, V. R. \& Sabey, J. B., WEMCO/Leeds flotation column development. In: Sastry, K. V. S. (Ed.), Column '88. Pheonix, Arizona, SME (1988). 
Dickens, A. W., Mackley, M. R. \& Williams, H. R., Experimental residence time distribution measurements for unsteady flow in baffled tubes. Chem. Eng. Sci., 77, 1471-1479 (1989).

Duan, J., Fornasiero, D. \& Ralston, J., Calculation of the flotation rate constant of chalcopyrite particles in an ore. Int. J. Miner. Process., 72, 227-237 (2003).

Engelbrecht, J. A. \& Woodburn, E. T., The effects of froth height, aeration rate and gas precipitation in flotation. J. SAIMM, 125-132 (1975).

Fabiyi, M. E. \& Skelton, B., The application of oscillatory flow mixing to photocatalytic wet oxidation. J. Photochem. Photobio. A: Chem., 129, 17-24 (1999).

Fangary, Y. S., Barigou, M., Seville, J. P. K. \& Parker, D. J., Fluid trajectories in a stirred vessel of non-Newtonian liquid using positron emission particle tracking. Chem. Eng. Sci., 55, 5969-5979 (2000).

Feng, D. \& Aldrich, C., Effect of particle size on the flotation performance of complex sulphide ores. Minerals Eng., 12, 721-731 (1999).

Finch, J. A., Column Flotation: A selected review - Part IV: Novel flotation devices. Minerals Eng., 8, 587-602 (1995).

Finch, J. A. \& Dobby, G. S., Column Flotation, Pergamon Press (1990).

Finch, J. A. \& Hardie, C. A., An example of innovation from the waste management industry: deinking flotation cells. Minerals Eng., 12, 476-475 (1999).

Fitch, A. W., Characterisation of flow in an oscillatory baffled column using digital particle image velocimetry and laser induced fluorescence. PhD Thesis, Herriot Watt, School of Physical Sciences and Engineering, Edinburgh (2003).

Fitch, A. W., Jian, H. \& Ni, X., An investigation of the effect of viscosity on mixing in an oscillatory baffled column using digital particle image velocimetry and computational fluid dynamics. Chem. Eng. J., 112, 197-210 (2005).

Franzidis, J. P. \& Manlapig, E. V., A comprehensive and useful model for flotation. In: Parekh, B. K. \& Miller, J. D. (Eds.) Advances in Flotation Technology. Littleton, CO, USA, Society for Mining, Metallurgy and Exploration, Inc. (1999).

Fuerstenau, M. C., Froth flotation: the first ninety years. In: Parekh, B. K. \& Miller, J. D. (Eds.) Advances in Flotation Technology. Littleton, CO, USA, Society for Mining, Metallurgy and Exploration, Inc. (1999). 
Gaidhani, H. K., McNeil, B. \& Ni, X., Production of pullulan using an oscillatory baffled bioreactor. J. Chem. Technol. Biotechnol., 78, 260-264 (2003).

Gao, P., Ching, W. H., Herrmann, M., Chan, C. K. \& Yue, P. L., Photooxidation of a model pollutant in an oscillatory flow reactor with baffles. Chem. Eng. Sci., 58, $1013-1020(2003)$.

Gao, S., Ni, X., Cumming, R. H., Greated, C. A. \& Norman, P., Experimental investigation of bentonite flocculation in a batch oscillatory baffled column. Sep. Sci. Technol., 33, 2143-2157 (1998).

Gaudin, A. M., Flotation, New York, McGraw-Hill (1957).

Gorain, B. K., Franzidis, J. P. \& Manlapig, E. V., Studies on the impeller type, impeller speed and air flow rate in an industrial scale flotation cell. Part 4: Effect of bubble surface area flux on flotation performance. Minerals Eng., 10, 367-379 (1997).

Gruber, G. A. \& Kelahan, M. E., Flotaire cell applications in phosphate flotation. In: Sastry, K. V. S. (Ed.), Column Flotation '88. Pheonix, Arizona, (1988).

Gu, X. \& Chiang, S.-H., A novel flotation column for oily water cleanup. Sep. Purific. Tech., 16, 193-203 (1999).

Hafez, M. M. \& Baird, M. H. I., Power consumption in a reciprocating plate extraction column. Trans IChemE, 56, 229-238 (1978).

Hafez, M. M. \& Prochazka, J., The dynamic effects in vibrating-plate and pulsed extractors - I. Theory and experimental technique. Chem. Eng. Sci., 29, 17451753 (1974).

Harris, M. C., Franzidis, J. P., O’Connor, C. T. \& Stonestreet, P., An evaluation of the role of particle size in the flotation of coal using different flotation technologies. Minerals Eng., 5, 1225-1238 (1992).

Harris, M. C., Runge, K. C., Whiten, W. J. \& Morrison, R. D., JKSimFloat as a practical tool for flotation process design and optimization. Proceedings of SME Mineral Processing Plant Design, Practice, and Control. Vancouver, SME (2002).

Harvey, A. P., Mackley, M. R. \& Stonestreet, P., Operation and optimization of an oscillatory flow continuous reactor. Ind. Eng. Chem. Res., 40, 5371-5377 (2001). 
He, M., Wang, Y. \& Forssberg, E., Slurry rheology in wet ultrafine grinding of industrial minerals: a review. Powder Tech., 147, 94-112 (2004).

Heiskanen, K., On the relationship between flotation rate constant and bubble surface area flux. Minerals Eng., 13, 141-149 (2000).

Hinze, J. O., Fundamentals of hydrodynamic mechanism of splitting in dispersion process. AIChE Journal, 1, 289-295 (1955).

Hinze, J. O., Turbulence, New York, McGraw-Hill (1959).

Hoatson, D. M., Jaireth S. \& Jaques, A. L., Nickel sulfide deposits in Australia: Characteristics, resources, and potential. Ore Geology Reviews, 29, 177-241 (2006).

Ityokumbul, M. T., de Aquino, J. A., O’Connor, C. T. \& Harris, M. C., Fine pyrite flotation in an agitated column cell. Int. J. Miner. Process., 58 (2000).

Jaeger, M. J., Diffusion and dispersion in steady counterflow a method for the separation of gases using enhanced mass transport in oscillatory flow. Chem. Eng. Sci., 53, 3613-3621 (1997).

Jameson, G. J., A new concept in flotation column design. In: Sastry, K. V. S. (Ed.), Column Flotation '88. Phoenix, Arizona, (1988).

Jameson, G. J., New concepts in coarse and ultrafines flotation. Flotation '07. Cape Town, MEi (2007).

Jameson, G. J., Nam, S. \& Moo-Young, M., Physical factors affecting recovery rates in flotation. Mineral. Sci. Eng., 9, 103-118 (1977).

Jealous, A. C. \& Johnson, H. F., Power requirements for pulse generation in pulse columns. Ind. Eng. Chem. Res., 47, 1159-1166 (1955).

Johnson, S. B., Franks, G. V., Scales, P. J., Boger, D. V. \& Healy, T. W., Surface chemistry - rheology relationships in concentrated mineral suspensions. Int. J. Miner. Process., 58, 267-304 (2000).

Karr, A. E., Performance of a reciprocating plate extraction column. AIChE Journal, 5, 446-452 (1959).

Kawatra, S. K. \& Eisele, T. C., The use of horizontal baffles to improve effectiveness of column flotation of coal. Proceedings of XVIII Int. Miner. Proc. Cong., Aus. $\operatorname{IMM}(1993)$. 
Knott, G. F. \& Mackley, M., On eddy motions near plates and ducts, induced by water waves and periodic flows. Phil. Trans. R. Soc. Lond., 294A (1412), 599-623 (1980).

Koh, P. T. L. \& Schwarz, M. P., CFD modelling of bubble-particle collision rates and efficiencies in a flotation cell. Minerals Eng., 16, 1055 - 1059 (2003).

Koh, P. T. L. \& Schwarz, M. P., CFD modelling of bubble-particle attachments in flotation cells. Minerals Eng., 19, 619-626 (2006).

Koh, P. T. L. \& Schwarz, M. P., Modelling attachment rates of multi-sized particles and bubbles in a flotation cell. Flotation '07. Cape Town, South Africa, MEi (2007).

Kolmogorov, A. N., Dissipation of energy in a locally isotropic turbulence. Doklady Akad. Nauk SSSR, 32, 141 (1941).

Kruis, F. E. \& Kusters, K. A., The collision rate of particles in turbulent flow. Chem. Eng. Comm., 158, 201-230 (1997).

Lee, C. A. \& Erickson, L. E., Bubble breakup and coalescence in turbulent gas-liquid dispersions. Chem. Eng. Comm., 59, 65-84 (1987).

Lee, C. T., Buswell, A. M. \& Middelberg, A. P. J., The influence of mixing on lysozyme renaturation during refolding in an oscillatory flow and a stirred-tank reactor. Chem. Eng. Sci., 57, 1679 - 1684 (2002).

Levenspiel, O., Chemical Reaction Engineering, New York, Wiley (1962).

Levesley, J. A. \& Bellhouse, B. J., The retention and suspension of particles in a fluid using oscillatory flow. Chem. Eng. Res. Des., 75, 288-297 (1997).

Levins, B. E. \& Glastonbury, J. R., Particle-liquid hydrodynamics and mass transfer in a stirred vessel; Part I - Particle-liquid motion. Chem. Eng. Res. Des., 50, 32-41 (1972).

Liepe, F. \& Mockel, H.-O., Untersuchungen zum stoffvererinigen in flussiger phase. Chem. Techn., 23, 231 (1976).

Lu, S. S., S. , Pan, Y. \& Gou, J., An approach to mineral particle -air bubble interaction in turbulent flow of flotation cell. Proceedings of the XIX Int. Miner. Proc. Cong. San Francisco, (1995).

Lynch, A. J., Johnson, N. W., Manlapig, E. V. \& Thorne, C. G., Developments in Mineral Processing - Mineral and Coal Flotation Circuits, New York, Elsevier Scientific Publishing Co. (1981). 
Mackley, M., Tweddle, G. M. \& Wyatt, I. D., Experimental heat transfer measurements for pulsatile flow in baffled tubes. Chem. Eng. Sci., 45, 1237-1242 (1990).

Mackley, M. R. \& Ni, X., Mixing and dispersion in a baffled tube for steady laminar and pulsatile flow. Chem. Eng. Sci., 46, 3139-3151 (1991).

Mackley, M. R. \& Ni, X., Experimental fluid dispersion measurements in periodic baffled tube arrays. Chem. Eng. Sci., 48, 3293-3305 (1993).

Mackley, M. R., Smith, K. B. \& Wise, N. P., The mixing and separation of particle suspensions using oscillatory flow in baffled tubes Trans. IChemE, 71, 649-656 (1993).

Mackley, M. R. \& Stonestreet, P., Heat transfer and associated energy dissipation for oscillatory flow in baffled tubes. Chem. Eng. Sci., 50, 2211-2224 (1995).

McKay, J. D., Foot, D. G. \& Shirts, M. B., Column flotation and bubble generation studies at the bureau of mines. In: Sastry, K. V. S. (Ed.), Column Flotation '88. Pheonix, Arizona, SME (1988).

Mignard, D., Amin, L. \& Ni, X., Population balance modelling of droplets in an oscillatory baffled reactor - using direct measurements of breakage rate constants. J. Chem. Technol. Biotechnol., 78, 364-369 (2003).

Mika, T. S. \& Fuerstenau, D. W., A microscopic model of the flotation process. Proceedings of the VIII Int. Miner. Proc. Cong. Leningrad, (1968).

Mohanty, M. K. \& Honaker, R. Q., A comparative evaluation of the leading advanced flotation technologies. Minerals Eng., 12, 1-13 (1999).

Moore, I. P. T. \& Cossor, G., Velocity distributions in a stirred tank containing a yield stress fluid. Chem. Eng. Sci., 50, 24672481 (1995).

Muhle, K. \& Domasch, K., Stability of particle aggregates in flocculation with polymers. Chemical Engineering Processing 29, 1-8 (1991).

Nel, E., Valenta, M. M., Coetzee, V. E. \& Kumalinga, N., The effect of high cleaner feed density due to upstream thickening on cleaner circuit performance. Flotation '07. Cape Town, MEi (2007).

Newell, R., Hydrodynamics and scale-up in Rushton turbine flotation cells. PhD, University of South Australia, Ian Wark Research Institute, (2006). 
Nguyen, A. V., George, P. \& Jameson, G. J., Demonstration of a minimum recovery of nanoparticles by flotation: Theory and experiment. Chem. Eng. Sci., 61, 24942509 (2006).

Nguyen, A. V. \& Schulze, H. J., Colloidal Science of Flotation, New York, Marcel Dekker Inc. (2004).

Ni, X., Cosgrove, J. A., Arnott, A. D., Greated, C. A. \& Cumming, R. H., On the measurement of strain rate in an oscillatory baffled column using particle image velocimetry. Chem. Eng. Sci., 55, 3195-3208 (2000).

Ni, X., Gao, S. \& Pritchard, D. W., A study of mass transfer in yeast in a pulsed baffled bioreactor. Biotechnol Bioeng, 45, 165-175 (1995).

Ni, X., Gao, S. \& Santangeli, L., On the effect of surfactant on mass transfer to waterglycerol solutions in a pulsed baffled reactor. J. Chem. Technol. Biotechnol., 69, 247 - 253 (1997).

Ni, X. \& Gough, P., On the discussion of the dimensionless groups governing oscillatory flow in a baffled tube. Chem. Eng. Sci., 52, 3209-3212 (1997).

Ni, X. \& Jian, H., A numerical study on scale-up behaviour in oscillatory baffled columns. Proceedings of the 7th World Congress of Chemical Engineering. Glasgow, (2005).

Ni, X. \& Mackley, M., Chemical reaction in batch pulsatile flow and stirred tank reactors. Chem. Eng. J., 52, 107-114 (1993).

Ni, X., Zhang, Y. \& Mustafa, I., Correlation of polymer particle size with droplet size in suspension polymerisation of methylmethacrylate in a batch oscillatory baffled reactor. Chem. Eng. Sci., 54, 841-850 (1999).

Oliveira, M. S. N., Fitch, A. W. \& Ni, X., A study of velocity and residence time of single bubbles in a gasssed oscillatory baffled column: effect of amplitude. $J$. Chem. Technol. Biotechnol., 78, 220-226 (2003).

Outotec (2008), Innovations in flotation technology, viewed 18 March 2008, $<$ http://www.outotec.com/pages/Page__36012.aspx $>$

Panton, R. L. \& Goldman, A. L., Correlation of non-linear orifice impedance. J. Acoust. Soc. Am., 60, 1390-1396 (1976).

Parthasarathy, P., Jameson G.J. \& N., A., Bubble Breakup in Stirred Vessels Predicting the Sauter Mean Diameter. Trans IChemE, 69, 295-301 (1991). 
Pyke, B., Bubble-particle capture in turbulent flotation systems. PhD Thesis, University of South Australia, Ian Wark Research Institute, Adelaide (2004).

Pyke, B., Fornasiero, D. \& Ralston, J., Bubble particle heterocoagulation under turbulent conditions. J. Colloidal Interface Sci., 265, 141-151 (2003).

Ralston, J., Fornasiero, D., Grano, S. R., Duan, J. \& Akroyd, T., Flotation rate constant prediction for metal sulphide particles. Centenary of Flotation Symposium. Brisbane, Australia, (2005).

Randall, E. W., Goodall, C. M., Fairlamb, P. M., Dold, P. L. \& O’Connor, C. T., A method for measuring bubbles in two- and three- phase systems. J. Phys E: Sci. Instrum., 22, 827-833 (1989).

Reade, W. C. \& Collins, L. R., Effect of preferential concentration on turbulent collision rates. Physics Fluids, 12, 2530-2540 (2000).

Rubinstein, J. B., Column Flotation: Processes, Designs, and Practices, CRC Press (1995).

Rubio, J., Souza, M. L. \& Smith, R. W., Overview of flotation as a wastewater treatment technique. Minerals Eng., 15, 139-155 (2002).

Rushton, J. H., Costich, E. W. \& Everett, H. J., Power characteristics of mixing impellers. Chem. Eng. Prog., 46, 395-404 (1950).

Saffman, P. G. \& Turner, J. S., On the collision of drops in turbulent clouds. J. Fluid Mech., 1, 16-30 (1956).

Savassi, O. N., Alexander, D. J., Franzidis, J.-P. \& Manlapig, E. V., An empirical model for entrainment in industrial flotation plants. Minerals Eng., 11, 243-256 (1998).

Schneider, J. C. \& Van Weert, G., Design and operation of the Hydrochem flotation column. In: Sastry, K. V. S. (Ed.), Column Flotation '88. Pheonix, Arizona, (1988).

Schubert, H., On the turbulence controlled microprocesses in flotation machines. Int. J. Miner. Process., 56, 257-276 (1999).

Schubert, H., On the optimization of hydrodynamics in fine particle flotation. Flotation '07. Cape Town, MEi (2007).

Schulze, H. J., Flotation as a heterocoagulation process: possibilities of calculating the probability of flotation In: Dobias, B. (Ed.) Coagulation and Flocculation: Theory and Applications. New York, Marcel Dekker (1993). 
Sege, G. \& Woodfield, F. W., Pulsed column variables. Chem Eng Prog, 50, 396-402 (1954).

Sherrel, I. M., Development of a flotation rate constant equation from first principles under turbulent flow conditions. PhD Thesis, Virginia Polytechnic Institute and State University, Mining and Minerals Engineering, Blacksburg, Virginia (2004).

Sherrel, I. M. \& Yoon, R. H., Development of a turbulent flotation model. Centenary of Flotation Symposium. Brisbane, Australia, (2005).

Skelton, B., Bustnes, T. E. \& Mackley, M. R., Development of the oscillatory flow based process for the production of biodiesel transport fuel. Proceedings of the 7th World Congress of Chemical Engineers. Glasgow, (2005).

Smith, R. W. \& Scott, J. L., Mechanisms of dodecylamine flotation of quartz. Min. Proc. Ext. Met. Review, 7, 81-94 (1990).

Smoluchowski, M., Versuch einer mathematischen theorie der koagulationskinetik kolloider losungen. Z. Phys. Chem., 92 (1917).

Stonestreet, P. \& Harvey, A. P., A mixing-based design methodology for continuous oscillatory flow reactors. Chem. Eng. Res. Des., 80, 31-44 (2002).

Sutherland, K. L., Kinetics of the flotation process. J. Phys. Coll., 52, 394-425 (1948).

Tangsathitkulchai, C. \& Austin, L. G., Rheology of concentrated slurries of particles of natural size distribution produced by grinding,. Powder Tech., 56, 293- 299. (1988).

Thomas, A. M. \& Narayanan, R., A comparison between the enhanced mass transfer in boundary and pressure driven oscillatory flow. Int. J. Heat Mass Transfer, 45, 4057-4062 (2002).

Tiitinen, J., Numerical modelling of a OK rotor-stator mixing device. Computer Aided Chemical Engineering, 14, 959-964 (2003).

Tooby, P. F., Wick, G. L. \& Isaacs, J. D., The motion of a small sphere in a rotating velocity field: A possible mechanism for suspending particles in turbulence. $J$. Geophys. Research, 82, 2096-2100 (1977).

Tucker, J. P., Deglon, D. A., Franzidis, J. P., Harris, M. C. \& O’Connor, C. T., An evaluation of a direct method of bubble size distribution measurement in a laboratory batch flotation cell. Minerals Eng., 7, 667-680 (1994). 
Ulan, W. W., Green, D. \& Kosick, G. A., In-plant testing of the Outokumpu high grade flotation cell. In: Agar, G. E., Huls, B. J. \& Hyma, D. B. (Eds.), Column Flotation '91. Sudbury, Ontario, Canada, (1991).

Valderrama, L. \& Rubio, J., High intensity conditioning and the carrier flotation of gold fine particles. Int. J. Miner. Process., 52, 273-285 (1999).

Vera, M. A., Franzidis, J. P. \& Manlipig, E. V., The JKMRC high bubble surface area flux flotation cell. Minerals Eng., 12, 477-484 (1999).

Weber, A., Walker, C., Redden, L., Lelinski, D. \& Ware, S., Scale-up and design of large-scale flotation equipment. In: Parekh, B. K. \& Miller, J. D. (Eds.) Advances in Flotation Technology. Littleton, CO, SME (1999).

Williams, J. J. E. \& Crane, R. I., Particle collision rate in turbulent flow. Int. J. Multiphase Flow, 9, 421 (1983).

Xinghau, C., Technical note: Study and test on a new flotation device; LM flotation cell. Minerals Eng., 11, 457-462 (1998).

Yang, D. C., A new packed column flotation cell. In: Sastry, K. V. S. (Ed.), Column Flotation '88. Pheonix, Arizona, SME (1988).

Ye, Y., Gopalakrishnan, S., Pacquet, E. \& Miller, J. D., Development of the air-sparged hydrocyclone. In: Sastry, K. V. S. (Ed.), Column Flotation '88. Pheonix, Arizona, SME (1988).

Yianatos, J. B., Fluid flow and kinetic modelling in flotation related processes: columns and mechanically agitated cells - A review. Chem. Eng. Res. Des., 85, 15911603 (2007).

Yianatos, J. B., Henríqueza, F. \& Tapiab, L., Evaluation of the largest flotation cells at Minera Los Pelambres. Flotation '07. Cape Town, MEi (2007).

Yoon, R. H., The role of hydrodynamic and surface forces in bubble-particle interaction. Int. J. Min. Proc., 58, 129-143 (2000).

Yoon, R. H., Adel, G. T. \& Luttrell, G. H., Apparatus and process for the separation of hydrophobic and hydrophilic particles using microbubble column flotation together with a process and apparatus for generation of microbubbles. US Patent $5,814,210$ (1998).

Yoon, R. H. \& Luttrell, G. H., The effect of bubble size on fine particle flotation. Min. Proc. Ext. Met. Review, 5, 101-122 (1989). 
Yoon, R. H. \& Mao, L., Application of extended DLVO theory IV: Derivation of flotation rate equation from first principles. J. Colloidal Interface Sci., 181, 613626 (1996).

Yuu, S., Collision rate of small particles in homogeneous and isotropic turbulence. AIChE Journal, 30 (1984). 


\section{Appendix A: Bubble Sizing Data}

Table A.1: Results of Bubble Size Analysis as function of Power Dissipation*

\begin{tabular}{|c|c|c|c|c|c|c|c|c|c|c|}
\hline $\begin{array}{c}\mathrm{x}_{\circ} \\
(\mathrm{mm})\end{array}$ & $\begin{array}{c}f \\
(\mathrm{~Hz})\end{array}$ & $\mathrm{Re}_{\circ}$ & $\begin{array}{c}\bar{\varepsilon} \\
\left(\mathrm{W} / \mathrm{m}^{3}\right)\end{array}$ & $\begin{array}{l}\text { No of } \\
\text { tests }\end{array}$ & \multicolumn{3}{|c|}{$\begin{array}{l}\mathrm{d} 10 \\
(\mathrm{~mm})\end{array}$} & \multicolumn{3}{|c|}{$\begin{array}{l}\mathrm{d} 32 \\
(\mathrm{~mm})\end{array}$} \\
\hline Column & Column & 0 & 0 & 3 & 0.62 & \pm & 0.03 & 1.21 & \pm & 0.06 \\
\hline 5 & 3 & 9425 & 17 & 3 & 0.69 & \pm & 0.01 & 1.29 & \pm & 0.11 \\
\hline 5 & 6 & 18850 & 139 & 3 & 0.58 & \pm & 0.03 & 1.27 & \pm & 0.02 \\
\hline 10 & 2 & 12566 & 28 & 3 & 0.61 & \pm & 0.04 & 1.27 & \pm & 0.07 \\
\hline 10 & 4.5 & 28274 & 319 & 3 & 0.58 & \pm & 0.05 & 1.24 & \pm & 0.11 \\
\hline 15 & 2.5 & 23562 & 156 & 6 & 0.62 & \pm & 0.02 & 1.14 & \pm & 0.10 \\
\hline 15 & 3.5 & 32987 & 427 & 3 & 0.52 & \pm & 0.01 & 1.17 & \pm & 0.06 \\
\hline 20 & 1 & 12566 & 21 & 3 & 0.70 & \pm & 0.03 & 1.22 & \pm & 0.11 \\
\hline 20 & 2.5 & 31416 & 334 & 6 & 0.56 & \pm & 0.04 & 1.12 & \pm & 0.04 \\
\hline 20 & 3 & 37699 & 578 & 3 & 0.59 & \pm & 0.04 & 1.18 & \pm & 0.12 \\
\hline 20 & 3.5 & 43982 & 918 & 3 & 0.58 & \pm & 0.03 & 1.05 & \pm & 0.03 \\
\hline 25 & 3 & 47124 & 1059 & 6 & 0.55 & \pm & 0.04 & 1.29 & \pm & 0.12 \\
\hline 25 & 3.5 & 54978 & 1682 & 3 & 0.54 & \pm & 0.03 & 1.10 & \pm & 0.21 \\
\hline
\end{tabular}

*All tests were conducted at a constant air rate of $21 / \mathrm{min}$, recycle rate of $13 \mathrm{l} / \mathrm{min}$ and frother dosage of $10 \mathrm{ppm}$ Dow 250. 


\section{Appendix B: Quartz Flotation Data}

Table B.1: Weakly Hydrophobic Quartz Flotation Results

\begin{tabular}{|c|c|c|c|}
\hline \multirow{8}{*}{ 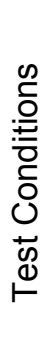 } & Collector Type & & HPYC \\
\hline & Frother Type & & Dowfroth 250 \\
\hline & $\%$ Solids & & $2.5 \%$ \\
\hline & $\mathrm{pH}$ & & 6.0 \\
\hline & Collector Dosage & $(\mathrm{mol} / \mathrm{l})$ & 1.56E-05 \\
\hline & Frother Dosage & (ppm) & 10.0 \\
\hline & $\mathrm{Jg}$ & $(\mathrm{cm} / \mathrm{s})$ & 0.12 \\
\hline & Recycle Rate & $(\mathrm{I} / \mathrm{min})$ & 6.0 \\
\hline
\end{tabular}

\begin{tabular}{|c|c|c|c|c|c|c|c|c|}
\hline \multirow{6}{*}{ 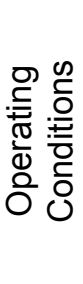 } & Amplitude & $(\mathrm{mm})$ & Column & 5 & 5 & 5 & 5 & 5 \\
\hline & Frequency & $(\mathrm{Hz})$ & Column & 1.0 & 1.5 & 2.0 & 2.5 & 3.0 \\
\hline & $\mathrm{Re}_{\circ}$ & & 0 & 3142 & 4712 & 6283 & 7854 & 9425 \\
\hline & Power Dissipation & $(\mathrm{W} / \mathrm{m} 3)$ & 0.0 & 0.6 & 2.2 & 5.2 & 10.1 & 17.5 \\
\hline & Residence Time & $(\min )$ & 6.0 & 5.3 & 5.8 & 6.0 & 5.9 & 5.8 \\
\hline & Gas Hold up & $(\%)$ & $1.2 \%$ & $1.3 \%$ & $1.4 \%$ & $1.7 \%$ & $2.3 \%$ & $1.7 \%$ \\
\hline \multirow{5}{*}{ 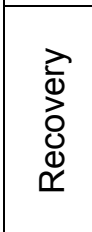 } & $<3$ & & $65.3 \%$ & $60.5 \%$ & $68.7 \%$ & $70.9 \%$ & $68.2 \%$ & $66.5 \%$ \\
\hline & $3-17$ & 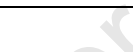 & $70.1 \%$ & $67.8 \%$ & $75.3 \%$ & $76.7 \%$ & $78.2 \%$ & $72.0 \%$ \\
\hline & $17-36$ & 21 & $89.8 \%$ & $92.0 \%$ & $93.9 \%$ & $94.5 \%$ & $95.1 \%$ & $91.9 \%$ \\
\hline & $36-76$ & - & $96.5 \%$ & $98.0 \%$ & $98.3 \%$ & $98.5 \%$ & $98.4 \%$ & $97.2 \%$ \\
\hline & $76-104$ & 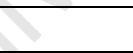 & $98.4 \%$ & $99.1 \%$ & $99.2 \%$ & $99.3 \%$ & $98.8 \%$ & $98.3 \%$ \\
\hline
\end{tabular}

\begin{tabular}{|c|c|c|c|c|c|c|c|c|}
\hline \multirow{6}{*}{ 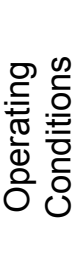 } & Amplitude & $(\mathrm{mm})$ & 10 & 10 & 25 & 25 & 25 & 25 \\
\hline & Frequency & $(\mathrm{Hz})$ & 0.5 & 1.0 & 1.5 & 2.0 & 2.5 & 3.0 \\
\hline & $\mathrm{Re}_{\mathrm{o}}$ & & 3142 & 6283 & 23562 & 31416 & 39270 & 47124 \\
\hline & Power Dissipation & $(\mathrm{W} / \mathrm{m} 3)$ & 0.4 & 3.5 & 133.2 & 315.8 & 616.8 & 1065.8 \\
\hline & Residence Time & $(\min )$ & 6.0 & 6.1 & 5.7 & 5.6 & 6.0 & 5.6 \\
\hline & Gas Hold up & $(\%)$ & $1.4 \%$ & $2.0 \%$ & $3.3 \%$ & $4.4 \%$ & $4.7 \%$ & $5.1 \%$ \\
\hline \multirow{5}{*}{ 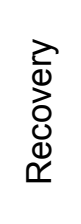 } & $<3$ & & $70.5 \%$ & $71.6 \%$ & $66.3 \%$ & $60.9 \%$ & $58.8 \%$ & $59.3 \%$ \\
\hline & $3-17$ & & $76.9 \%$ & $77.9 \%$ & $74.1 \%$ & $67.6 \%$ & $68.8 \%$ & $69.0 \%$ \\
\hline & $17-36$ & & $94.2 \%$ & $95.1 \%$ & $91.0 \%$ & $85.2 \%$ & $85.2 \%$ & $81.8 \%$ \\
\hline & $36-76$ & & $98.5 \%$ & $98.6 \%$ & $94.6 \%$ & $89.3 \%$ & $88.2 \%$ & $83.7 \%$ \\
\hline & $76-104$ & & $99.4 \%$ & $99.4 \%$ & $95.5 \%$ & $91.3 \%$ & $89.1 \%$ & $84.7 \%$ \\
\hline
\end{tabular}


Table B.2: Moderately Hydrophobic Quartz Flotation Results for Column Flotation

\begin{tabular}{|c|c|c|c|}
\hline \multirow{8}{*}{ 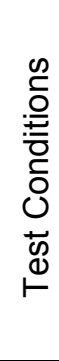 } & Collector Type & & Dodecylamine \\
\hline & Frother Type & & Dowfroth 250 \\
\hline & \% Solids & & $1.0 \%$ \\
\hline & $\mathrm{pH}$ & & 7.0 \\
\hline & Collector Dosage & $(\mathrm{mol} / \mathrm{l})$ & 1.0E-04 \\
\hline & Frother Dosage & (ppm) & 10.0 \\
\hline & $\mathrm{Jg}$ & $(\mathrm{cm} / \mathrm{s})$ & 0.12 \\
\hline & Recycle Rate & $(\mathrm{l} / \mathrm{min})$ & 6.0 \\
\hline
\end{tabular}

\begin{tabular}{|c|c|c|c|c|c|c|c|c|}
\hline \multirow{7}{*}{ 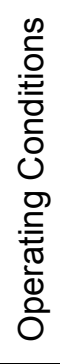 } & Amplitude & $(\mathrm{mm})$ & Column & Column & Column & Column & Mean & $\begin{array}{l}\text { Std } \\
\text { Dev }\end{array}$ \\
\hline & Frequency & $(\mathrm{Hz})$ & 0 & 0 & 0 & 0 & 0 & 0 \\
\hline & $\mathrm{Re}_{\mathrm{o}}$ & & 0 & 0 & 0 & 0 & 0 & 0 \\
\hline & Power Input & $(\mathrm{W} / \mathrm{m} 3)$ & 0.0 & 0.0 & 0.0 & 0 & 0 & 0 \\
\hline & Gas Hold-up & $(\%)$ & $1.4 \%$ & $1.3 \%$ & $1.3 \%$ & $0.8 \%$ & $1.2 \%$ & $0.3 \%$ \\
\hline & Residence Time & $(\min )$ & 3.0 & 3.1 & 3.1 & 3.1 & 3.1 & 0.1 \\
\hline & $\mathrm{N}$ & A & 3.00 & 3.00 & 3.00 & 3.00 & 3.00 & 0 \\
\hline \multirow{5}{*}{ 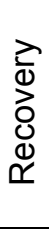 } & $<3$ & 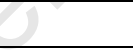 & $58.3 \%$ & $56.0 \%$ & $53.3 \%$ & $56.3 \%$ & $56.0 \%$ & $2.0 \%$ \\
\hline & $3-17$ & & $58.8 \%$ & $55.9 \%$ & $54.8 \%$ & $56.7 \%$ & $56.6 \%$ & $1.7 \%$ \\
\hline & $17-36$ & & $85.8 \%$ & $84.6 \%$ & $84.2 \%$ & $83.7 \%$ & $84.6 \%$ & $0.9 \%$ \\
\hline & $36-76$ & & $96.4 \%$ & $96.2 \%$ & $96.1 \%$ & $95.5 \%$ & $96.0 \%$ & $0.4 \%$ \\
\hline & $76-104$ & & $99.0 \%$ & $98.9 \%$ & $99.0 \%$ & $98.6 \%$ & $98.9 \%$ & $0.2 \%$ \\
\hline \multirow{5}{*}{ 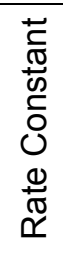 } & $<3$ & $\left(\mathrm{~min}^{-1}\right)$ & 0.34 & 0.30 & 0.28 & 0.30 & 0.31 & 0.03 \\
\hline & $3-17$ & $\left(\mathrm{~min}^{-1}\right)$ & 0.35 & 0.30 & 0.29 & 0.31 & 0.31 & 0.02 \\
\hline & $17-36$ & $\left(\mathrm{~min}^{-1}\right)$ & 0.92 & 0.84 & 0.81 & 0.79 & 0.84 & 0.06 \\
\hline & $36-76$ & $\left(\mathrm{~min}^{-1}\right)$ & 2.03 & 1.90 & 1.87 & 1.73 & 1.88 & 0.12 \\
\hline & $76-104$ & $\left(\mathrm{~min}^{-1}\right)$ & 3.60 & 3.44 & 3.56 & 3.05 & 3.41 & 0.25 \\
\hline
\end{tabular}


Table B.3: Moderately Hydrophobic Quartz Flotation Results at 5 mm Amplitude

\begin{tabular}{|c|c|c|c|}
\hline \multirow{8}{*}{ 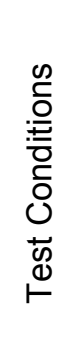 } & Collector Type & & Dodecylamine \\
\hline & Frother Type & & Dowfroth 250 \\
\hline & $\%$ Solids & & $1.0 \%$ \\
\hline & $\mathrm{pH}$ & & 7.0 \\
\hline & Collector Dosage & $(\mathrm{mol} / \mathrm{l})$ & $1.0 \mathrm{E}-04$ \\
\hline & Frother Dosage & (ppm) & 10.0 \\
\hline & $\mathrm{Jg}$ & $(\mathrm{cm} / \mathrm{s})$ & 0.12 \\
\hline & Recycle Rate & $(1 / \min )$ & 6.0 \\
\hline
\end{tabular}

\begin{tabular}{|c|c|c|c|c|c|c|}
\hline \multirow{7}{*}{ 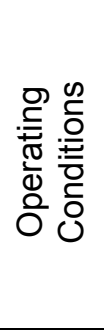 } & Amplitude & $(\mathrm{mm})$ & 5 & 5 & 5 & 5 \\
\hline & Frequency & $(\mathrm{Hz})$ & 3.01 & 3.07 & 3.86 & 5.22 \\
\hline & $\mathrm{Re}_{\mathrm{o}}$ & & 9461 & 9636 & 12130 & 16415 \\
\hline & Power Input & $(\mathrm{W} / \mathrm{m} 3)$ & 17.3 & 18.1 & 35.9 & 116.6 \\
\hline & Gas Hold-up & $(\%)$ & $2.9 \%$ & $2.2 \%$ & $3.6 \%$ & $4.3 \%$ \\
\hline & Residence Time & $(\min )$ & 2.6 & 2.7 & 2.7 & 2.8 \\
\hline & $\mathrm{N}$ & & 4.52 & 4.52 & 4.67 & 4.39 \\
\hline \multirow{5}{*}{ 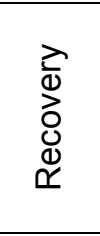 } & $<3$ & & $69.2 \%$ & $58.7 \%$ & $70.5 \%$ & $69.8 \%$ \\
\hline & $3-17$ & & $67.0 \%$ & $63.8 \%$ & $65.0 \%$ & $65.6 \%$ \\
\hline & $17-36$ & & $90.3 \%$ & $90.7 \%$ & $92.3 \%$ & $92.3 \%$ \\
\hline & $36-76$ & & $97.8 \%$ & $98.2 \%$ & $98.4 \%$ & $98.2 \%$ \\
\hline & $76-104$ & & $99.6 \%$ & $99.5 \%$ & $99.6 \%$ & $99.4 \%$ \\
\hline \multirow{5}{*}{ 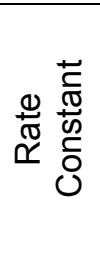 } & $<3$ & $\left(\min ^{-1}\right)$ & 0.51 & 0.36 & 0.52 & 0.50 \\
\hline & $3-17$ & $\left(\min ^{-1}\right)$ & 0.48 & 0.42 & 0.44 & 0.44 \\
\hline & $17-36$ & $\left(\min ^{-1}\right)$ & 1.16 & 1.14 & 1.27 & 1.25 \\
\hline & $36-76$ & $\left(\min ^{-1}\right)$ & 2.28 & 2.37 & 2.49 & 2.38 \\
\hline & $76-104$ & $\left(\min ^{-1}\right)$ & 4.14 & 3.78 & 3.99 & 3.43 \\
\hline
\end{tabular}


Table B.4: Moderately Hydrophobic Quartz Flotation Results at 10 mm Amplitude

\begin{tabular}{|c|c|c|c|}
\hline \multirow{8}{*}{ 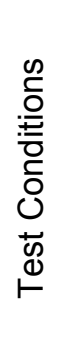 } & Collector Type & & Dodecylamine \\
\hline & Frother Type & & Dowfroth 250 \\
\hline & $\%$ Solids & & $1.0 \%$ \\
\hline & $\mathrm{pH}$ & & 7.0 \\
\hline & Collector Dosage & $(\mathrm{mol} / \mathrm{l})$ & $1.0 \mathrm{E}-04$ \\
\hline & Frother Dosage & (ppm) & 10.0 \\
\hline & $\mathrm{Jg}$ & $(\mathrm{cm} / \mathrm{s})$ & 0.12 \\
\hline & Recycle Rate & $(1 / \mathrm{min})$ & 6.0 \\
\hline
\end{tabular}

\begin{tabular}{|c|c|c|c|c|c|c|}
\hline \multirow{7}{*}{ 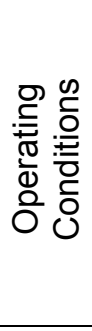 } & Amplitude & $(\mathrm{mm})$ & 10 & 10 & 10 & 10 \\
\hline & Frequency & $(\mathrm{Hz})$ & 1.44 & 1.44 & 1.76 & 1.76 \\
\hline & $\mathrm{Re}_{\mathrm{o}}$ & & 9055 & 9059 & 11061 & 11071 \\
\hline & Power Input & (W/m3) & 11.0 & 11.5 & 19.9 & 21.2 \\
\hline & Gas Hold-up & $(\%)$ & $2.9 \%$ & $2.2 \%$ & $2.4 \%$ & $2.3 \%$ \\
\hline & Residence Time & $(\min )$ & 2.6 & 2.6 & 2.7 & 2.6 \\
\hline & $\mathrm{N}$ & & 4.40 & 4.40 & 4.24 & 4.24 \\
\hline \multirow{5}{*}{ 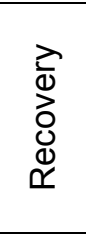 } & $<3$ & & $61.7 \%$ & $59.4 \%$ & $63.7 \%$ & $59.5 \%$ \\
\hline & $3-17$ & & $63.2 \%$ & $60.4 \%$ & $66.4 \%$ & $61.7 \%$ \\
\hline & $17-36$ & & $89.3 \%$ & $88.8 \%$ & $91.3 \%$ & $89.2 \%$ \\
\hline & $36-76$ & & $97.7 \%$ & $97.8 \%$ & $98.4 \%$ & $97.6 \%$ \\
\hline & $76-104$ & & $99.2 \%$ & $99.5 \%$ & $99.7 \%$ & $99.2 \%$ \\
\hline \multirow{5}{*}{ 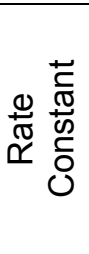 } & $<3$ & $\left(\mathrm{~min}^{-1}\right)$ & 0.41 & 0.38 & 0.42 & 0.38 \\
\hline & $3-17$ & $\left(\mathrm{~min}^{-1}\right)$ & 0.43 & 0.39 & 0.46 & 0.41 \\
\hline & $17-36$ & $\left(\mathrm{~min}^{-1}\right)$ & 1.11 & 1.08 & 1.21 & 1.11 \\
\hline & $36-76$ & $\left(\mathrm{~min}^{-1}\right)$ & 2.26 & 2.31 & 2.59 & 2.25 \\
\hline & $76-104$ & $\left(m^{-1}\right)$ & 3.35 & 3.88 & 4.56 & 3.45 \\
\hline
\end{tabular}

\begin{tabular}{|c|c|c|c|c|c|}
\hline \multirow{7}{*}{ 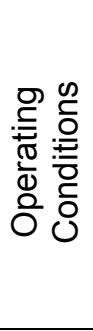 } & Amplitude & $(\mathrm{mm})$ & 10 & 10 & 10 \\
\hline & Frequency & $(\mathrm{Hz})$ & 2.18 & 2.96 & 3.75 \\
\hline & $\mathrm{Re}_{\mathrm{o}}$ & & 13712 & 18622 & 23592 \\
\hline & Power Input & $(\mathrm{W} / \mathrm{m} 3)$ & 36.9 & 100.2 & 225.2 \\
\hline & Gas Hold-up & $(\%)$ & $3.0 \%$ & $3.6 \%$ & $4.4 \%$ \\
\hline & Residence Time & $(\min )$ & 2.7 & 2.7 & 2.7 \\
\hline & $\mathrm{N}$ & & 4.10 & 3.74 & 3.05 \\
\hline \multirow{5}{*}{ 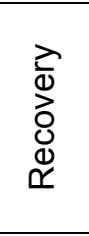 } & $<3$ & & $64.0 \%$ & $56.8 \%$ & $55.8 \%$ \\
\hline & $3-17$ & & $66.7 \%$ & $67.0 \%$ & $65.1 \%$ \\
\hline & $17-36$ & & $91.5 \%$ & $91.5 \%$ & $89.8 \%$ \\
\hline & $36-76$ & & $98.3 \%$ & $97.5 \%$ & $95.9 \%$ \\
\hline & $76-104$ & & $99.6 \%$ & $98.7 \%$ & $96.9 \%$ \\
\hline \multirow{5}{*}{ 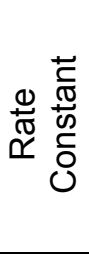 } & $<3$ & $\left(\min ^{-1}\right)$ & 0.44 & 0.35 & 0.35 \\
\hline & $3-17$ & $\left(\min ^{-1}\right)$ & 0.47 & 0.48 & 0.47 \\
\hline & $17-36$ & $\left(\min ^{-1}\right)$ & 1.26 & 1.30 & 1.26 \\
\hline & $36-76$ & $\left(\min ^{-1}\right)$ & 2.63 & 2.36 & 2.09 \\
\hline & $76-104$ & $\left(\min ^{-1}\right)$ & 4.51 & 3.08 & 2.42 \\
\hline
\end{tabular}


Table B.5: Moderately Hydrophobic Quartz Flotation Results at 25 mm Amplitude

\begin{tabular}{|c|c|c|c|}
\hline \multirow{8}{*}{ 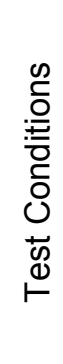 } & Collector Type & & Dodecylamine \\
\hline & Frother Type & & Dowfroth 250 \\
\hline & $\%$ Solids & & $1.0 \%$ \\
\hline & $\mathrm{pH}$ & & 7.0 \\
\hline & Collector Dosage & $(\mathrm{mol} / \mathrm{l})$ & $1.0 \mathrm{E}-04$ \\
\hline & Frother Dosage & (ppm) & 10.0 \\
\hline & $\mathrm{Jg}$ & $(\mathrm{cm} / \mathrm{s})$ & 0.12 \\
\hline & Recycle Rate & $(1 / \min )$ & 6.0 \\
\hline
\end{tabular}

\begin{tabular}{|c|c|c|c|c|c|c|}
\hline \multirow{7}{*}{ 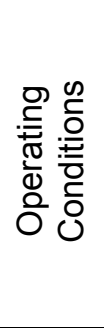 } & Amplitude & $(\mathrm{mm})$ & 25 & 25 & 25 & 25 \\
\hline & Frequency & $(\mathrm{Hz})$ & 0.45 & 0.46 & 0.46 & 0.46 \\
\hline & $\mathrm{Re}_{\mathrm{o}}$ & & 7078 & 7299 & 7239 & 7229 \\
\hline & Power Input & $(\mathrm{W} / \mathrm{m} 3)$ & 3.6 & 4.1 & 4.0 & 4.0 \\
\hline & Gas Hold-up & $(\%)$ & $1.5 \%$ & $1.4 \%$ & $1.3 \%$ & $1.6 \%$ \\
\hline & Residence Time & $(\min )$ & 2.8 & 2.7 & 2.7 & 2.7 \\
\hline & $\mathrm{N}$ & & 4.31 & 4.31 & 4.31 & 4.31 \\
\hline \multirow{5}{*}{ 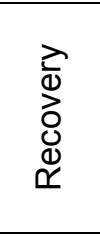 } & $<3$ & & $54.1 \%$ & $52.2 \%$ & $48.7 \%$ & $55.0 \%$ \\
\hline & $3-17$ & & $56.0 \%$ & $51.3 \%$ & $50.6 \%$ & $55.3 \%$ \\
\hline & $17-36$ & & $85.2 \%$ & $82.4 \%$ & $82.6 \%$ & $84.6 \%$ \\
\hline & $36-76$ & & $96.5 \%$ & $95.9 \%$ & $95.9 \%$ & $96.5 \%$ \\
\hline & $76-104$ & & $99.2 \%$ & $99.0 \%$ & $98.9 \%$ & $99.2 \%$ \\
\hline \multirow{5}{*}{ 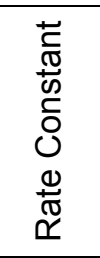 } & $<3$ & $\left(\min ^{-1}\right)$ & 0.31 & 0.30 & 0.27 & 0.33 \\
\hline & $3-17$ & $\left(\min ^{-1}\right)$ & 0.33 & 0.29 & 0.29 & 0.33 \\
\hline & $17-36$ & $\left(\min ^{-1}\right)$ & 0.87 & 0.79 & 0.81 & 0.88 \\
\hline & $36-76$ & $\left(\min ^{-1}\right)$ & 1.84 & 1.75 & 1.79 & 1.91 \\
\hline & $76-104$ & $\left(\min ^{-1}\right)$ & 3.17 & 3.00 & 3.04 & 3.33 \\
\hline
\end{tabular}

\begin{tabular}{|c|c|c|c|c|c|c|c|}
\hline \multirow{7}{*}{ 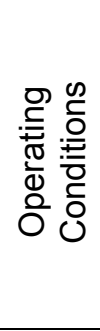 } & Amplitude & $(\mathrm{mm})$ & 25 & 25 & 25 & 25 & 25 \\
\hline & Frequency & $(\mathrm{Hz})$ & 0.59 & 0.76 & 0.97 & 1.33 & 1.67 \\
\hline & $\mathrm{Re}_{\mathrm{o}}$ & & 9307 & 11969 & 15237 & 20894 & 26222 \\
\hline & Power Input & $(\mathrm{W} / \mathrm{m} 3)$ & 8.9 & 19.6 & 40.2 & 99.7 & 190.6 \\
\hline & Gas Hold-up & $(\%)$ & $1.8 \%$ & $1.5 \%$ & $2.2 \%$ & $2.9 \%$ & $3.5 \%$ \\
\hline & Residence Time & $(\min )$ & 2.7 & 2.7 & 2.7 & 2.7 & 2.7 \\
\hline & $\mathrm{N}$ & & 3.92 & 3.83 & 3.48 & 3.15 & 2.88 \\
\hline \multirow{5}{*}{ 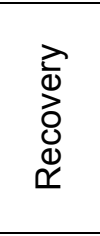 } & $<3$ & & $54.5 \%$ & $55.0 \%$ & $56.2 \%$ & $59.8 \%$ & $54.6 \%$ \\
\hline & $3-17$ & & $58.3 \%$ & $56.8 \%$ & $61.0 \%$ & $62.9 \%$ & $62.0 \%$ \\
\hline & $17-36$ & & $87.5 \%$ & $85.8 \%$ & $88.8 \%$ & $89.1 \%$ & $88.5 \%$ \\
\hline & $36-76$ & & $97.3 \%$ & $96.4 \%$ & $97.3 \%$ & $96.9 \%$ & $95.9 \%$ \\
\hline & $76-104$ & & $99.4 \%$ & $98.8 \%$ & $99.2 \%$ & $98.7 \%$ & $97.8 \%$ \\
\hline \multirow{5}{*}{ 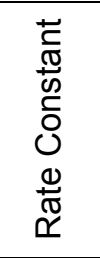 } & $<3$ & $\left(\min ^{-1}\right)$ & 0.32 & 0.33 & 0.34 & 0.39 & 0.34 \\
\hline & $3-17$ & $\left(\min ^{-1}\right)$ & 0.36 & 0.34 & 0.40 & 0.43 & 0.43 \\
\hline & $17-36$ & $\left(\min ^{-1}\right)$ & 1.02 & 0.93 & 1.13 & 1.18 & 1.21 \\
\hline & $36-76$ & $\left(\min ^{-1}\right)$ & 2.21 & 1.95 & 2.36 & 2.35 & 2.21 \\
\hline & $76-104$ & $\left(\min ^{-1}\right)$ & 3.97 & 3.05 & 3.94 & 3.42 & 2.98 \\
\hline
\end{tabular}




\section{Appendix C: Nickel Ore Slimes Flotation}

Table C.1: Normalised High Rheology Flotation Results

\begin{tabular}{|c|c|c|c|}
\hline \multirow{6}{*}{ 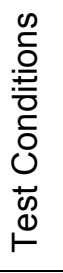 } & $\%$ Solids & & 11.2 \\
\hline & Slurry Density & $(\mathrm{kg} / \mathrm{m} 3)$ & 1196 \\
\hline & Yield Stress & $(\mathrm{Pa})$ & 5.4 \\
\hline & Viscosity & $(\mathrm{cP})$ & 7.7 \\
\hline & $\mathrm{Jg}$ & $(\mathrm{cm} / \mathrm{s})$ & 0.64 \\
\hline & Recycle Rate & $(\mathrm{l} / \mathrm{min})$ & 17.0 \\
\hline
\end{tabular}

\begin{tabular}{|c|c|c|c|c|c|c|c|c|}
\hline \multirow{6}{*}{ 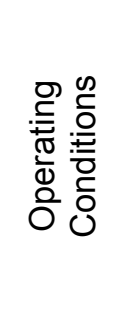 } & Amplitude & $(\mathrm{mm})$ & 25 & 25 & 25 & 25 & 25 & 25 \\
\hline & Frequency & $(\mathrm{Hz})$ & 0.80 & 1.07 & 1.37 & 1.85 & 2.32 & 2.66 \\
\hline & $\mathrm{Re}_{\mathrm{o}}$ & & 1942 & 2610 & 3341 & 4515 & 5654 & 6496 \\
\hline & Power Input & $(\mathrm{W} / \mathrm{m} 3)$ & 21 & 55 & 114 & 321 & 716 & 1193 \\
\hline & Gas Hold-up & $(\%)$ & $21.8 \%$ & $23.9 \%$ & $30.0 \%$ & $26.3 \%$ & $24.6 \%$ & $27.2 \%$ \\
\hline & Residence Time & $(\min )$ & 2.92 & 3.28 & 3.24 & 2.90 & 3.09 & 3.13 \\
\hline \multirow{4}{*}{ 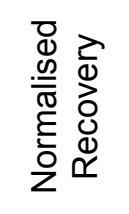 } & $\mathrm{Ni}$ & 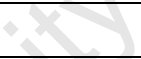 & 0.97 & 0.96 & 1.00 & 0.85 & 0.77 & 0.63 \\
\hline & $\mathrm{MgO}$ & 8 & 0.16 & 0.14 & 0.18 & 0.14 & 0.14 & 0.11 \\
\hline & 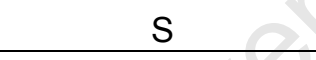 & & 0.56 & 0.70 & 0.63 & 0.40 & 0.42 & 0.31 \\
\hline & $\mathrm{Fe}$ & & 0.19 & 0.19 & 0.21 & 0.18 & 0.18 & 0.14 \\
\hline \multirow{4}{*}{ 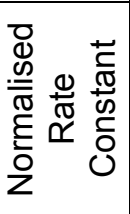 } & $\mathrm{Ni}$ & & 1.08 & 0.94 & 1.00 & 0.91 & 0.76 & 0.59 \\
\hline & $\mathrm{MgO}$ & & 0.14 & 0.11 & 0.14 & 0.12 & 0.11 & 0.09 \\
\hline & $\mathrm{s}$ & & 0.55 & 0.64 & 0.57 & 0.38 & 0.37 & 0.26 \\
\hline & $\mathrm{Fe}$ & & 0.17 & 0.15 & 0.17 & 0.16 & 0.15 & 0.11 \\
\hline
\end{tabular}


Table C.2: Normalised Low Rheology Flotation Results

\begin{tabular}{|c|c|c|c|}
\hline \multirow{6}{*}{ 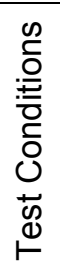 } & $\%$ Solids & & 4.3 \\
\hline & Slurry Density & $(\mathrm{kg} / \mathrm{m} 3)$ & 1075 \\
\hline & Yield Stress & $(\mathrm{Pa})$ & 0.0 \\
\hline & Viscosity & $(\mathrm{cP})$ & 6.3 \\
\hline & $\mathrm{Jg}$ & $(\mathrm{cm} / \mathrm{s})$ & 0.64 \\
\hline & Recycle Rate & $(1 / \min )$ & 17.0 \\
\hline
\end{tabular}

\begin{tabular}{|c|c|c|c|c|c|c|c|c|}
\hline \multirow{6}{*}{ 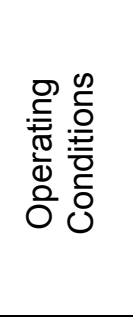 } & Amplitude & $(\mathrm{mm})$ & 25 & 25 & 25 & 25 & 25 & 25 \\
\hline & Frequency & $(\mathrm{Hz})$ & 0.79 & 1.07 & 1.36 & 1.85 & 2.34 & 2.66 \\
\hline & $\mathrm{Re}_{\mathrm{o}}$ & & 1938 & 2608 & 3330 & 4520 & 5720 & 6500 \\
\hline & Power Input & (W/m3) & 20 & 53 & 118 & 347 & 630 & 1328 \\
\hline & Gas Hold-up & $(\%)$ & $24.6 \%$ & $25.3 \%$ & $26.5 \%$ & $26.5 \%$ & $35.4 \%$ & $30.1 \%$ \\
\hline & Residence Time & $(\min )$ & 3.2 & 3.4 & 3.3 & 3.5 & 2.9 & 3.8 \\
\hline \multirow{4}{*}{ 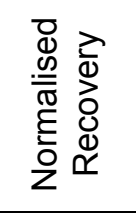 } & $\mathrm{Ni}$ & & 0.97 & 0.95 & 1.00 & 0.89 & 0.72 & 0.83 \\
\hline & $\mathrm{MgO}$ & & 0.16 & 0.16 & 0.23 & 0.20 & 0.11 & 0.22 \\
\hline & $S$ & & 0.59 & 0.65 & 0.66 & 0.70 & 0.38 & 0.62 \\
\hline & $\mathrm{Fe}$ & & 0.22 & 0.19 & 0.25 & 0.22 & 0.13 & 0.22 \\
\hline \multirow{4}{*}{ 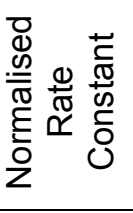 } & $\mathrm{Ni}$ & & 0.99 & 0.91 & 1.00 & 0.82 & 0.75 & 0.69 \\
\hline & $\mathrm{MgO}$ & & 0.13 & 0.12 & 0.18 & 0.15 & 0.09 & 0.15 \\
\hline & $S$ & & 0.54 & 0.56 & 0.60 & 0.61 & 0.36 & 0.49 \\
\hline & $\mathrm{Fe}$ & & 0.18 & 0.15 & 0.20 & 0.16 & 0.11 & 0.16 \\
\hline
\end{tabular}




\section{Appendix D: Derivation of Equation 2.26}

According to the acoustic model, the instantaneous power consumption in $\mathrm{OBC}$ is given by the following equation (c.f. equation 2.25):

$$
J_{\text {acoustic }}=\frac{\pi \cdot D^{2}}{4}\left(\rho \cdot\left(\frac{Z \cdot K_{i}}{S}\right) \cdot x_{o}^{2} \cdot \omega^{3} \cdot \cos (\omega \cdot t) \cdot \sin (\omega \cdot t)+\frac{3 \cdot n \cdot \rho \cdot l}{S} \cdot x_{o}^{2} \cdot \omega^{3} \cdot\left|\sin ^{3}(\omega \cdot t)\right|\right)
$$

The time-averaged power consumption is then given by the integral of Equation D.1 over one cycle of oscillation, viz.

$\bar{J}_{\text {acoustic }}=\frac{\int_{0}^{T} J_{\text {acoustic }} \cdot d t}{T}$

By substituting equation D.1 in equation D.2 and noting that the integral of $\cos (\omega t) \cdot \sin (\omega t)$ over one cycle of oscillation is zero, we obtain:

$\bar{J}_{\text {acoustic }}=\frac{\pi D^{2}}{4} \cdot \frac{3 \rho \cdot n \cdot l \cdot x_{o}^{2} \cdot \omega^{3}}{S} \cdot \frac{\int_{0}^{T}\left|\sin ^{3}(\omega \cdot t)\right| \cdot d t}{T}$

The value of the period of oscillation, $\mathrm{T}$ is given by $2 \pi / \omega$. Therefore:

$\bar{J}_{\text {acoustic }}=\frac{3 \pi \cdot \rho \cdot n \cdot D^{2} \cdot l \cdot x_{o}^{2} \cdot \omega^{3}}{4 \cdot S} \cdot \frac{2 \int_{0}^{(\pi / \omega)} \sin ^{3}(\omega \cdot t) \cdot d t}{(2 \pi / \omega)} \quad$ (by symmetry)

Now, $\int \sin ^{3}(\omega \cdot t) \cdot d t=\frac{\cos (3 \omega \cdot t)-9 \cos (\omega \cdot t)}{12 \omega}$ 


$$
\begin{aligned}
\therefore \int_{0}^{(\pi / \omega)} \sin ^{3}(\omega t) \cdot d t & =\frac{\cos \left(3 \omega \cdot \frac{\pi}{\omega}\right)-9 \cos \left(\omega \cdot \frac{\pi}{\omega}\right)}{12 \omega}-\frac{\cos (0)-9 \cos (0)}{12 \omega} \\
& =\frac{\cos (3 \pi)-9 \cos (\pi)}{12 \omega}+\frac{8}{12 \omega} \\
& =\frac{8}{12 \omega}+\frac{8}{12 \omega} \\
& =\frac{4}{3 \omega}
\end{aligned}
$$

Substituting this relationship into equation D.4, we obtain:

$$
\bar{J}_{\text {acoustic }}=\frac{3 \pi \cdot \rho \cdot n \cdot D^{2} \cdot l \cdot x_{o}^{2} \cdot \omega^{3}}{4 \cdot S} \cdot \frac{\omega}{\pi} \cdot \frac{4}{3 \omega}
$$

$\therefore \bar{J}_{\text {acoustic }}=\frac{\rho \cdot n \cdot D^{2} \cdot l \cdot x_{o}^{2} \cdot \omega^{3}}{S}$ as given in equation 2.26 . 